\title{
Origine et radiation initiale des chauves-souris modernes: nouvelles découvertes dans l'Éocène d'Afrique du Nord
}

\author{
Anthony RAVEL \\ Laboratoire de Paléontologie, Institut des Sciences de l'Évolution de Montpellier \\ (ISE-M, UMR 5554, CNRS/UM2/IRD/EPHE), c.c. 064, \\ Université de Montpellier, place Eugène Bataillon, F-34095 Montpellier cedex 05 (France) \\ anthony.ravel@univ-montp2.fr \\ Mohammed ADACI \\ Mustapha BENSALAH \\ Laboratoire de recherche $n^{\circ} 25$, Département des Sciences de la Terre, \\ Université Abou Bekr Belkaïd, b.p. 119, Tlemcen 13000 (Algérie)
}

Anne-Lise CHARRUAULT Laboratoire de Paléontologie, Institut des Sciences de l'Évolution de Montpellier (ISE-M, UMR 5554, CNRS/UM2/IRD/EPHE), c.c. 064, Université de Montpellier, place Eugène Bataillon, F-34095 Montpellier cedex 05 (France)

El Mabrouk ESSID

Hayet Khayati AMMAR

Wissem MARZOUGUI

Office national des Mines (ONM), 24 rue 8601, 2035 La Charguia, b.p. 215, 1080 Tunis (Tunisie)

Mohammed MAHBOUBI Laboratoire de Paléontologie stratigraphique et Paléoenvironnement, Université d'Oran, b.p. 1524, El M'naouer, Oran 31000 (Algérie)

Fateh MEBROUK

Département des Sciences de la Terre, Faculté des Sciences, Université de Jijel, b.p. 98, Ouled Aissa, 18000 Jijel (Algérie)

Gilles MERZERAUD Géosciences Montpellier (UMR-CNRS 5243), c.c. 060, Université Montpellier 2, Place Eugène Bataillon, F-34095 Montpellier cedex 05 (France)

Monique VIANEY-LIAUD Rodolphe TABUCE Laurent MARIVAUX

Laboratoire de Paléontologie, Institut des Sciences de l'Évolution de Montpellier (ISE-M, UMR 5554, CNRS/UM2/IRD/EPHE), c.c. 064, Université de Montpellier, place Eugène Bataillon, F-34095 Montpellier cedex 05 (France)

Publié le 30 septembre 2016

urn:Isid:zoobank.org:pub:FC07ACBE-03F7-414A-BB64-1BB0711766BF

Ravel A., Adaci M., Bensalah M., Charruault A.-L., Essid E. M., Ammar H. K., Marzougui W., Mahboubi M., Mebrouk F., Merzeraud G., Vianney-Liaud M., Tabuce R. \& Marivaux L. 2016. - Origine et radiation initiale des chauves-souris modernes: nouvelles découvertes dans l'Éocène d'Afrique du Nord. Geodiversitas 38 (3): 355-434. http://dx.doi.org/10.5252/g2016n3a3 
MOTS CLÉS

Chiroptera,

radiation,

Éocène,

Afrique du Nord, cladistique

paléobiogéographie,

genres nouveaux espèces nouvelles.

KEY WORDS

Chiroptera,

radiation,

Eocene,

North Africa,

cladistics,

paleobiogeography,

new genera,

new species.

\section{RÉSUMÉ}

Cette étude intègre des faunes inédites de chiroptères fossiles découvertes lors de plusieurs campagnes de terrain réalisées en Afrique du Nord. Il s'agit de localités fossiliferes datées de l'Éocène inférieur à moyen de Tunisie (Chambi) et d'Algérie (Glib Zegdou). Les différentes analyses systématiques et cladistiques réalisées sur ce matériel fossile, essentiellement constitué de dents isolées, ont permis d'apporter de nombreux éclaircissements sur les modalités évolutives de la radiation des premiers microchiroptères modernes. Ces nouvelles faunes ont livré pas moins de huit nouveaux taxons répartis dans cinq familles modernes bien identifiées: un Necromantidae (?Necromantis fragmentum Ravel, n. sp.), deux Hipposideridae Miller, 1907 (?Palaeophyllophora tunisiensis Ravel, n. sp. et Hipposideros (Pseudorhinolophus) africanum Ravel, n. sp.), trois Emballonuridae Gervais in de Castelnau, 1855 (Vespertiliavus kasserinensis Ravel, n. sp., ?Vespertiliavus aenigma Ravel, n. sp., et Pseudovespertiliavus parva Ravel n. gen., n. sp.), un Nycteridae (Khoufechia gunnelli Ravel n. gen., n. sp.) ainsi qu'un Vespertilionidae indéterminé. Deux autres taxons sont également répertoriés (Chambinycteris pusilli Ravel n. gen., n. sp. et Drakonycteris glibzegdouensis Ravel n. gen., n. sp.), mais leur morphologie dentaire originale ne permet pas de les attribuer de manière formelle à des familles connues. Deux analyses cladistiques permettent de clarifier les positions phylogénétiques des taxons les mieux documentés. Par ailleurs, elles mettent en évidence l'existence d'un axe majeur de dispersion des chiroptères Hipposideridae et Emballonuridae depuis l'Afrique du Nord vers le Sud de l'Europe durant l'Éocène moyen.

\section{ABSTRACT}

Origin and radiation of modern bats: new discoveries in the Eocene of North Africa.

This study focuses on new fossil faunas of bats discovered in North Africa following several field campaigns. The fossiliferous localities include those from the Early to Middle Eocene of Tunisia (Chambi) and Algeria (Glib Zegdou). Systematic and cladistic analyses carried out on the fossil material, primarily including isolated teeth, allow us to highlight the modalities of the radiation of the first modern microbats. These new faunas include eight well-identified new taxa belonging to five modern families: one Necromantidae (?Necromantis fragmentum Ravel, n. sp.), two Hipposideridae Miller, 1907 (?Palaeophyllophora tunisiensis Ravel, n. sp. and Hipposideros [Pseudorhinolophus] africanum Ravel, n. sp.), three Emballonuridae Gervais in de Castelnau, 1855 (Vespertiliavus kasserinensis Ravel, n. sp., ?Vespertiliavus aenigma Ravel, n. sp., and Pseudovespertiliavus parva Ravel n. gen., n. sp.), one Nycteridae (Khoufechia gunnelli Ravel n. gen., n. sp.) and an undetermined Vespertilionidae. Two other taxa are identified (Chambinycteris pusilli Ravel n. gen., n. sp. and Drakonycteris glibzegdouensis Ravel n. gen., n. sp.), but they show an original dental pattern which precludes a family attribution. Two cladistic analyses allow to clarify the phylogenetic position of the best documented taxa. The results highlight for Hipposideridae and Emballonuridae chiropterans a major dispersal axis from North Africa towards South Europe during the Middle Eocene.

\section{EXTENDED ABSTRACT}

Today, the order Chiroptera is one of the most diversified placental mammalian groups. The special aspect of bats is their ability to fly and to echolocate, two key adaptations which allow them to migrate over long distances and to colonize exclusive ecological niches. The initial radiation of bat, described as explosive, involves primitive Eocene families ("Eochiroptera" sensu Van Valen [1979]) that are found in all continents except Antarctica. Almost simultaneously, several representatives of the two main modern groups of Chiroptera (i.e., Rhinolophoidea and Vespertilionoidea) occur in the late Early-early Middle Eocene of Tunisia (Chambi). This fossil record of Chiroptera shows that the oldest modern families are only documented in Africa as early as the late Early Eocene while they appear only during the middle Middle Eocene in Europe, North America and Asia, and during the late Eocene of Australia. This highlights the critical role of the Afro-Arabian continent in the emergence and initial radiation of modern bat groups during the early Cenozoic.

The Afro-Arabian Paleogene fossil record of Chiroptera is so far limited to few localities mainly situated in Northern regions of Africa. The oldest bat of the continent was discovered in the Early Eocene of El Kohol in Algeria. This archaic bat displays primitive dental features shared with the "Eochiroptera". Another presumably archaic bat, Tanzanycteris mannardi Gunnell, Jacobs, Herendeen, Head, Kowalski, Msuya, Mizambwa, Harrison, Habersetzer \& Storch, 2003 (Tanzanycteridae), is documented by a nearly complete skeleton in the Middle Eocene of Mahenge (Tanzania). To the exception of these two taxa, the majority of Paleogene bats found both in Arabia and Africa belong to the main extant bat groups. The fossils of the Eocene of Algeria and Tunisia document a representative of the Rhinolophoidea and two species of the Philisidae, which are considered as primitive extinct Vespertilionoidea. Separated by over 10 million years, a diversified modern bat assemblage occurs in the Late Eocene (Quarry BQ-2 and L-41) to the Early Oligocene (Quarry I) of the Fayum in Egypt. The Egyptian bat assemblages comprise six modern families: Rhinopomatidae Bonaparte, 1838, Emballonuridae Gervais in de Castelnau, 1855, Megadermatidae Allen, 1864, Myzopodidae Thomas, 1904, 
Philisidae Sigé, 1985, and Vespertilionidae Gray, 1821). Two other families (Hipposideridae Miller, 1907 and Nycteridae Van Der Hoeven, 1855) are testified in the Early Oligocene of Taqah (Sultanat of Oman). Some fragmentary teeth are also described in the middle Middle Eocene of Aznag (Morocco), which show a modern dental morphology, but the scarcity of the material does not allow for a precise systematic attribution. Our current knowledge of the fossil record testifies to the high diversity of modern bats in Africa and Arabia since the end of the Early Eocene. But how this diversity did take place? Is Africa the place of the origin and radiation of modern bats? What are the lineages concerned?

This study includes new bat fossil faunas recently recovered from several field seasons in two Eocene fossiliferous formations in Tunisia (Chambi) and Algeria (Glib Zegdou). The Algerian bat fossils were found in the HG50 and HGL50' localities (HGL for Hammada Gour Lazib) of the Glib Zegdou outlier, a Paleogene continental section located in the Gour Lazib complex in the Sahara of Western Algeria (Hammada du Dra). The Djebel Chambi is situated in the Kasserine area, in the Central Western part of Tunisia. Most of the material of this study comes from three localities (CBI-1, CBI-2, and CBI-3) of the fluvio-lacustrine deposits situated at the base of the continental deposits of Chambi. Considering their temporal and geographic proximity, fossils from Chambi and Glib Zegdou are considered to belong to the same biogeographic Province. The common charophytes and mammals recorded from Chambi (locus CBI-1) and Glib Zegdou (loci HGL50 and HGL50') testify to the temporal proximity of both sites, dating from the late Early to early Middle Eocene. The indurated limestone blocs of CBI-1, CBI-2 and CBI-3, and the carbonated sandstones of HGL50 and HGL50' were treated by acid processing (acetic acid 10\%) and screen washings of several hundred kilograms of sediments. More than 500 specimens were collected. The material is essentially constituted by isolated teeth and mandible fragments. Only the best preserved and most informative specimens were included in this study (289 specimens). The pictures of specimens were taken with Scanning Electron Microscope housed at the "Centre de Ressources en Imagerie cellulaire de Montpellier" (CRIC-M).

The comparative material used for the taxonomic and systematic analyses derives from fossil and extant bat collections of the "Institut des Sciences de l'Évolution de Montpellier" (ISE-M), "Université de Montpellier" (UM), which were gathered by Bernard Sigé and François Catzeflis. The comparative material was completed by specimens coming from fossil and extant bat collections of the "Muséum national d'Histoire naturelle" (MNHN) in Paris. In this paper we describe eight well-identified new taxa belonging to five modern families. In addition to the Philisidae already described in Chambi and Glib Zegdou, this bat assemblage includes now the oldest representatives of several extant families: two species of Hipposideridae (?Palaeophyllophora tunisiensis Ravel, n. sp. and Hipposideros [Pseudorhinolophus] africanum Ravel, n. sp.), three Emballonuridae (Vespertiliavus kasserinensis Ravel, n. sp., ?Vespertiliavus aenigma Ravel, n. sp., and Pseudovespertiliavus parva Ravel n. gen., n. sp.), one Nycteridae (Khoufechia gunnelli Ravel n. gen., n. sp.), and an undetermined Vespertilionidae, which is documented only by fragmentary myotodont lower molars. The largest species of the Chambi bat fauna is a Necromantidae (?Necromantis fragmentum Ravel, n. sp.). The robust dental morphology of this taxon, its necromantodont lower molars and special features of its upper molars, are shared with extinct genus Necromantis Weithofer, 1887 from the Middle Eocene of the Quercy Phosphorites in South Western France. Chambinycteris pusilli Ravel n. gen., n. sp. shares dental and mandible characteristics found in primitive Rhinolophoidea and Emballonuroidea. This original combination of characters and the lack of most informative material do not allow to precise its familial attribution. The last species, Drakonycteris glibzedouensis Ravel n. gen., n. sp., documented by five specimens found in HGL50, displays a dental morphology which is somewhat unique among bat. This new species suggests the presence of an extinct unknown bat family, which was probably endemic of Africa as the Philisidae. The taxonomic composition of bats in Chambi, notably the presence of ?Necromantis, Palaeophyllophora Revilliod, 1922, Hippopsideros (Pseudorhinolophus) Schlosser, 1887 and Vespertiliavus Schlosser, 1887 is similar to the bat fauna characterizing the Middle Eocene localities of the Quercy Phosphorites, France. These affinities suggest faunal exchanges between North Africa and South Europe during the Middle Eocene, exchanges which involved Hipposideridae, Emballonuridae and Necromantidae.

Two cladistics analyses were realized in order to clarify the phylogenetic positions of the best documented African fossils within each respective family. The selected characters and character states were established essentially on the dental remains and on dentary/maxillary. For both analyses, the ingroup included modern bat taxa. Characters were polarized via the out-group comparison method, using three archaic bats found in the Early-Middle Eocene of Europe: Icaronycteris menui Russell, Louis \& Savage, 1973; Archaeonycteris brailloni Russell, Louis \& Savage, 1973; Palaeochiropteryx tupaiodon Revilliod, 1917. Data matrices were managed using NDE software (Nexus Data Editor v. 0.5.0). Phylogenetic reconstructions were performed with PAUP v.4.0 beta 10 Win with an exact search for the most parsimonious tree ("Branch and Bound" option [BandB]). Clade robustness was evaluated with the Bremer Index and Bootstrap percentages. Matrices and character descriptions for each analysis are put in the Appendixes of this paper. The first cladistic analysis was a dental assessment 
of to position of the Hipposideridae, ?Palaeophyllophora tunisiensis Ravel, n. sp. and Hipposideros (Pseudorhinolophus) africanum Ravel, n. sp., within the "Hipposideridae + Rhinolophidae" clade. The analysis yielded only one most parsimonious tree of 129 steps with consistency index (CI) of 0.488 and retention index (RI) of 0.683 . Our phylogenetic reconstruction places both Tunisian taxa within the Hipposideridae: ?Palaeophyllophora tunisiensis Ravel, n. sp. at the base of Palaeophyllophora clade and Hipposideros (Pseudorhinolophus) africanum Ravel, n. sp. among the Hipposideros clade. These results highlight the affinities between African and European Hipposideridae, which suggest the dispersal of this family into South Europe from Africa during the Middle Eocene (owing the greater antiquity of African forms). Interestingly, our study did not revealed a representative of Rhinolophidae in Tunisia and Algeria localities despite the diversity of modern bats. The discovery of Protorhinolophus shanghuangensis Ravel, Marivaux, Qi, Wang \& Beard, 2014 in the Middle Eocene of Shanghuang (China) suggested the westward dispersion from Eastern Asia toward Europe, implying only Rhinolophidae. If we consider the oldest occurrence of Hipposideridae in North Africa and the oldest occurrence of Rhinolophidae in the Eastern Asia, a common origin for these two families in Central Asia is so far the most parsimonious scenario. However the current Paleogene bat fossil record lacking in this area cannot support this hypothesis.

The second cladistic analysis included the three Emballonuridae species Vespertiliavus kasserinensis Ravel, n. sp., ?Vespertiliavus aenigma Ravel, n. sp., and Pseudovespertiliavus parva Ravel n. gen., n. sp., the Nycteridae Khoufechia gunnelli Ravel n. gen., n. sp., and the enigmatic Chambinycteris pusilli Ravel n. gen., n. sp. The PAUP analysis yielded three equally most-parsimonious trees of 132 steps each, with consistency index (CI) of 0.576 and retention index (RI) of 0.743 . The Emballonuridae and Nycteridae form two natural groups in all topologies. The phylogenetic trees differ only in the position of Chibanycteris herberti Sigé, Thomas, Sen, Gheerbrant, Roger \& Al-Sulaimani, 1994 within the Nycteridae. Our results confirm the basal position of $K$. gunnelli Ravel n. gen., n. sp. and Chambinycteris pusilli Ravel n. gen., n. sp. within the Nycteridae and Emballonuridae, respectively. The new genus Pseudovespertiliavus Ravel, n. gen. from Glib Zegdou is branched between Tachypteron franzeni Storch, 2002 (the Emballonuridae found in the Middle Eocene of Messel) and the paraphyletic group of Vespertiliavus, which includes the two species identified in Chambi (?Vespertiliavus aenigma Ravel, n. sp. and Vespertiliavus kasserinensis Ravel, n. sp.). These results highlight the close relationships between the Vespertiliavus species from the Middle Eocene of the Quercy Phosphorites and those from the EarlyMiddle Eocene of Chambi. The most likely scenario suggested by these results would be in favor of a dispersal event of the Emballonuridae from North Africa toward South of Europe during the Middle Eocene. In contrast, the Nycteridae, as the Philisidae, seems to have maintained a distribution limited to the Afro-Arabian continent, an assumption which could indicate an African origin for this family.

The new bat assemblage from the Early-Middle Eocene of Tunisia and Algeria described in this study includes ten new species belonging to five modern families. Such a high diversity demonstrates the early radiation in Africa of the three main extant bat groups (i.e., Rhinolophoidea, Emballonuroidea, and Vespertilionoiodea). A phylogenetic approach highlights a major North-South axe of dispersion for Necromantidae, Hipposideridae and Emballonuridae, from North Africa to Europe. These dispersals events took place during the Middle Eocene. Taphonomy can be a good indicator in paleoecology and habitation of bat fossils. It is worth noting that if basal Chiroptera ("Eochiroptera") are often found in fluvio-lacustrine deposits, modern forms are in contrast primarily found in karstic deposits, which could indicate that they inhabited caves. The accumulation of unexpected species richness in fluviolacustrine deposits of Chambi suggests a hydrological transport from ancient cave system, which was used for roosting for many microbat colonies. But such richness, very restricted in Chambi, would also have led to strong interspecific competition, which was probably an important factor for the events of radiation and dispersion.

\section{INTRODUCTION}

Au sein des mammifères placentaires actuels, les chiroptères (Chiroptera Blumenbach, 1779) représentent l'ordre le plus diversifié après les rongeurs (par ex. : Simmons 2005a, b). Une telle réussite évolutive a de toute évidence été largement favorisée par leur capacité au vol battu et à l'écholocation, deux innovations adaptatives clés qui leur ont permis de coloniser rapidement des niches écologiques exclusives (Simmons 2005b). Le registre fossile cénozoïque des chiroptères s'étage sur près de 55 millions d'années (Eiting \& Gunnell 2009). Plusieurs lignées de chiroptères sont apparues subitement au début de l'Éocène et presque simultanément sur plusieurs continents, traduisant ainsi une première phase de radiation expansive pour l'ordre. Les plus anciens chiroptères sont attestés dans des localités datées de l'Éocène 


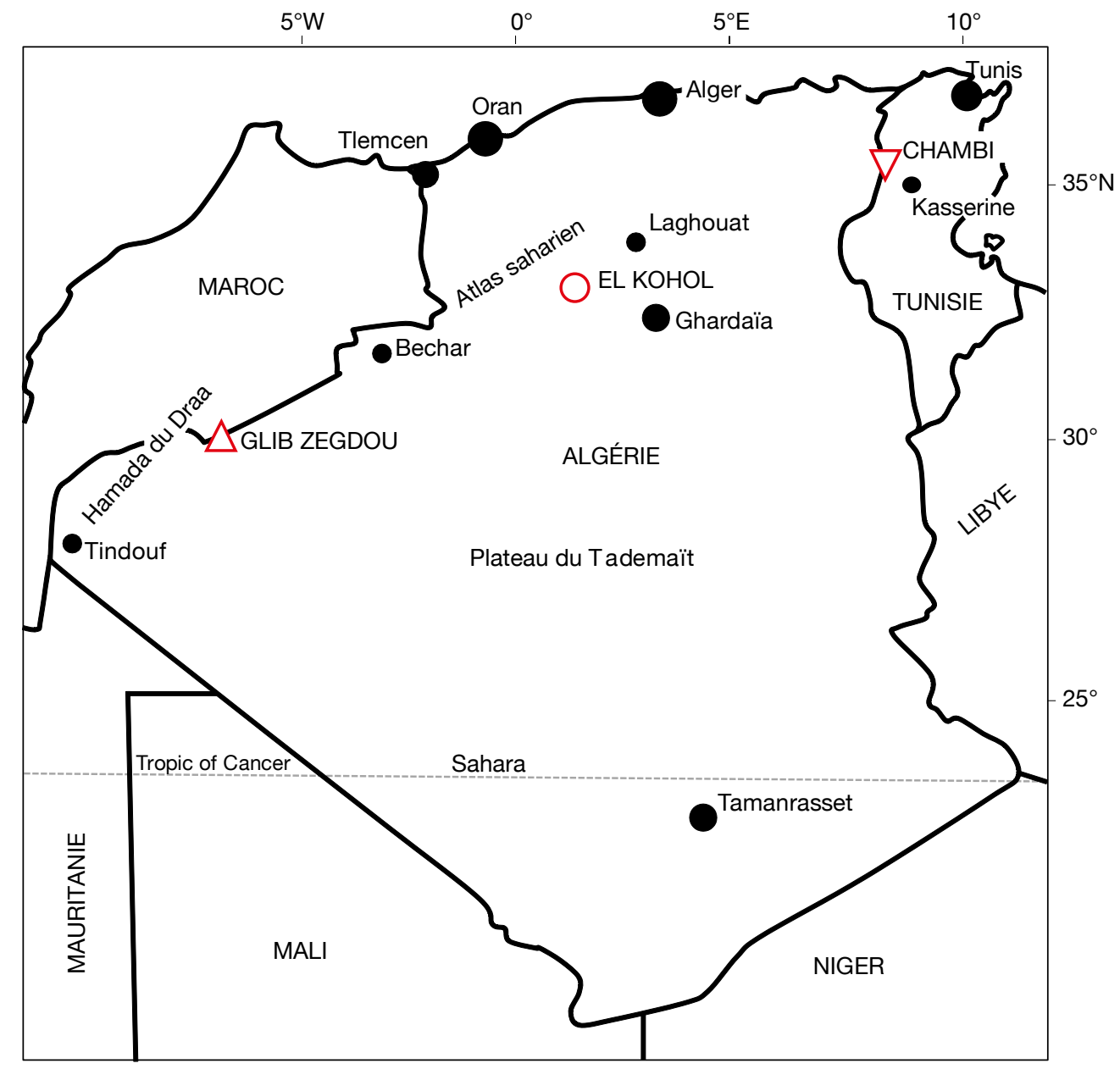

FIG. 1. - Localisation géographique des localités de Chambi $(\boldsymbol{\nabla})$ en Tunisie et du Glib Zegdou ( $\Delta$ ) en Algérie. Chambi est situé dans le parc national du Djebel Chambi environ à $15 \mathrm{~km}$ à l'Ouest de Kasserine. Le Glib Zegdou est situé environ à $15 \mathrm{~km}$ à l'Est de l'aire des Gour Lazib dans les Hammada du Dra (Sahara de l'Ouest algérien). El Kohol $(\bigcirc)$ est situé dans la région de Brézina dans le Sud-Est des montagnes de l'Atlas. Cette localité datée de l'Éocène inférieur a révélé le plus ancien chiroptère d'Afrique (Ravel et al. 2011).

inférieur en Amérique du Nord et du Sud (par ex. : Beard et al. 1992 ; Simmons \& Geisler 1998 ; Tejedor et al. 2005 ; Czaplewski et al. 2008 ; Simmons et al. 2008 ; Smith et al. 2012), en Europe (par ex. : Russell et al. 1973; Sigé 1991a; Tabuce et al. 2009a; Smith et al. 2012), en Afrique (par ex. : Sigé 1991b; Ravel et al. 2011, 2012, 2015), en Inde (Smith et al. 2007, 2012), et en Australie (Hand et al. 1994). Bien que faiblement documentée, d'un point de vue taxinomique, la diversité des chiroptères basaux durant l'Éocène est toutefois remarquable puisqu'ils sont répartis entre six familles (Onychonycteridae Simmons, Seymour, Habersetzer \& Gunnell, 2008, Icaronycteridae Jepsen, 1966, Archaeonycteridae Revilliod, 1917, Hassianycteridae Habersetzer \& Storch, 1987, Palaeochiropterygidae Revilliod, 1917 et Tanzanycteridae Gunnell, Jacobs, Herendeen, Head, Kowalski, Msuya, Mizambwa, Harrison, Habersetzer \& Storch, 2003). Ces formes archaïques, également appelées "Eochiroptera» sensu Van Valen (1979), n'appartiendraient à aucun des grands ensembles modernes de chiroptères, et seraient pour la plupart des groupes-souches du clade Chiroptera (Simmons \& Geisler 1998; Smith et al. 2012).
Si ces fossiles semblent documenter la première phase de radiation impliquant des lignées avortées de chiroptères, qu'en est-il de l'origine des formes modernes ? Plus de la moitié des 30 familles de chiroptères recensées (éteintes et actuelles) sont reconnues dès l'Éocène, une diversité qui témoigne ainsi d'une véritable radiation explosive. Au cours de l'Éocène, en plus des "Eochiroptera ", on retrouve également les représentants de huit familles actuelles (Pteropodidae Gray, 1821, Rhinolophidae Gray, 1825, Rhinopomatidae Bonaparte, 1838, Hipposideridae Miller, 1907, Megadermatidae Allen, 1864, Emballonuridae Gervais in de Castelnau, 1855, Molossidae Gill, 1872, et Vespertilionidae Gray, 1821). À l'Éocène inférieur, le registre fossile des chiroptères montre que les plus anciennes familles modernes ne sont documentées qu'en Afrique alors qu'en Europe, en Amérique du Nord et en Asie, elles ne sont attestées qu'à partir de l'Éocène moyen, et à l'Éocène supérieur en Australie (Gunnell \& Simmons 2005; Eiting \& Gunnell 2009). Cela met en évidence le rôle important de l'Afrique au début du Cénozoïque dans l'émergence et la dispersion des groupes modernes de chauve-souris. 


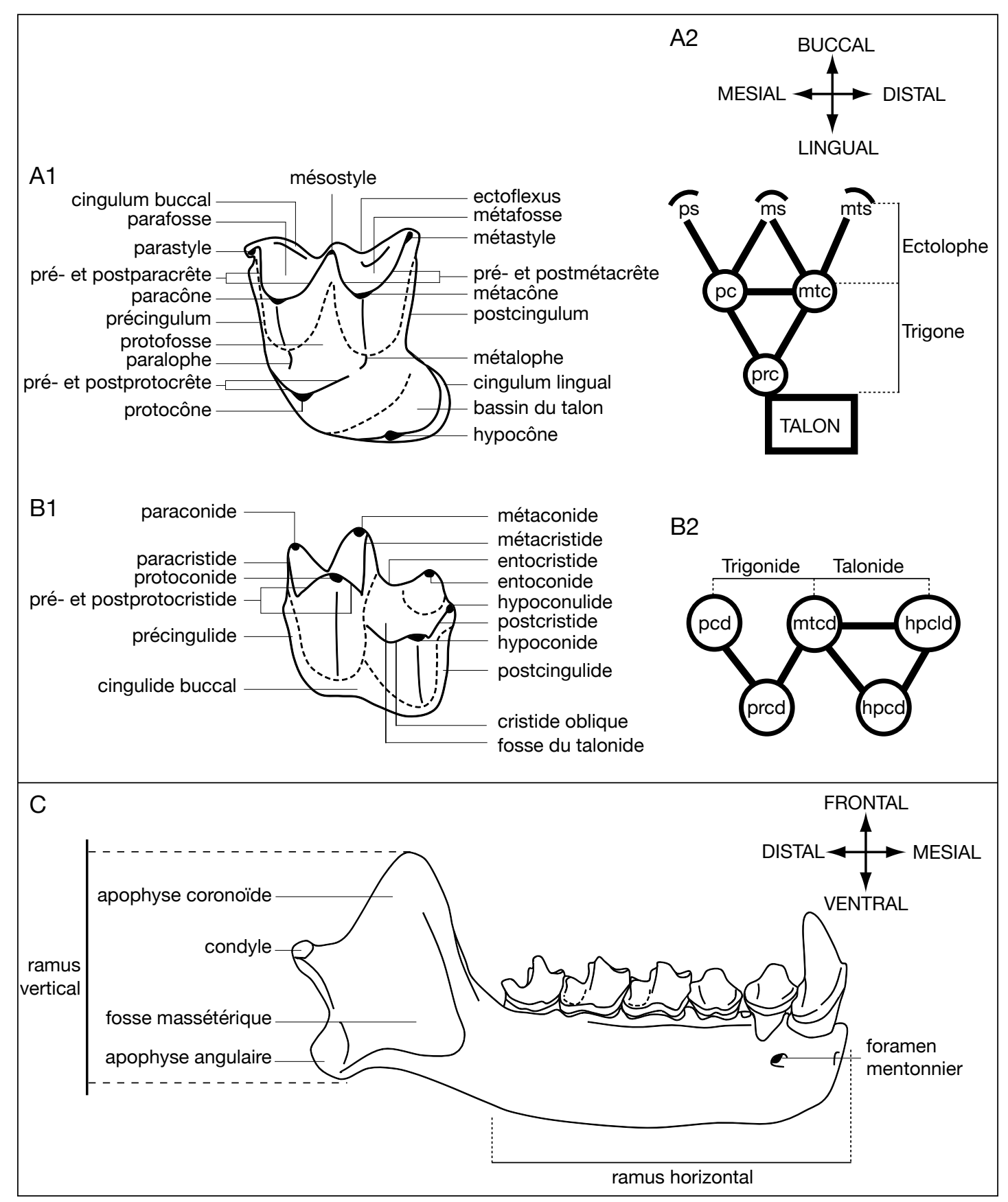

FIG. 2. - Terminologie des molaires et du dentaire: A1, terminologie de la molaire supérieure; A2, schéma simplifié de la structuration de la molaire supérieure; B1, terminologie de la molaire inférieure; B2, schéma simplifié de la structuration de la molaire inférieure; $\mathbf{C}$, terminologie du dentaire.

Jusqu'ici, les informations disponibles sur les chiroptères du Paléogène d'Afrique étaient encore très fragmentaires et se limitaient aux taxons de l'Éocène inférieur d'El Kohol en Algérie (Ravel et al. 2011), de l'Éocène inférieur terminal - Éocène moyen basal de Chambi en Tunisie (Sigé 1991b; Ravel et al. 2012, 2015) et du Glib Zegdou en Algérie (Ravel et al. 2015), de l'Éocène moyen de Mahenge en Tanzanie (Gunnell et al. 2003) et d'Aznag au Maroc (Tabuce et al. 2005), de l'Éocène supérieur et de l'Oligocène inférieur du Fayum en Égypte (Sigé 1985; Gunnell et al.
2008, 2014), et de l'Oligocène inférieur de Taqah au Sultanat d'Oman (Sigé et al. 1994). À l'exception de la forme primitive découverte à El Kohol (Ravel et al. 2011), la grande majorité des chiroptères du Paléogène d'Afrique appartient aux grands ensembles modernes. Les fossiles éocènes de Tunisie et d'Algérie documentent un représentant des Rhinolophoidea et deux espèces appartenant aux Vespertilionoidea (Philisidae Sigé, 1985; Ravel et al. 2012, 2015), alors qu'à la même époque ne sont présents sur les continents nordiques que les chiroptères archaïques (Gun- 
nell \& Simmons 2005; Smith et al. 2012). Plus de 10 millions d'années séparent le gisement de Chambi en Tunisie des premiers gisements du Fayoum en Égypte (niveau BQ-2, L-41, et I). Dans les carrières BQ-2 et L-41 datés de l'Éocène supérieur, en plus des Philisidae (Gunnell et al. 2008, 2014), sont présents cinq familles modernes de chiroptères (Rhinopomatidae, Emballonuridae, Megadermatidae, Vespertilionidae, et Myzopodidae Thomas, 1904). À l'Oligocène, deux autres familles de chiroptères modernes (Hipposideridae et Nycteridae Van Der Hoeven, 1855) s'ajoutent au registre arabo-africain sur la base de fossiles récoltés au Sultanat d'Oman (Sigé et al. 1994). Les chiroptères modernes sont dès lors particulièrement bien documentés en Afrique au Paléogène. Comment s'est mise en place cette diversité ? L'Afrique est-elle à l'origine des groupes modernes qui se retrouvent ultérieurement dans la Province Holarctique?

Les recherches paléontologiques de terrain ont été effectuées dans l'Éocène d'Afrique du Nord (Algérie [Gour Lazib] et de Tunisie [Chambi]). Elles ont permis de récolter de nombreux restes fossiles attribuables à plusieurs taxons de chiroptères. Ces campagnes de fouilles ont été réalisées dans le cadre d'une collaboration scientifique réunissant l'équipe du laboratoire de paléontologie de l'Institut des Sciences de l'Evolution de Montpellier (ISE-M), France, des géologues de l'Office National des Mines (ONM) de Tunis, et des paléontologues/géologues des universités de Tlemcen, Oran et Jijel en Algérie. Les fossiles proviennent de nouvelles localités fossilifères, récemment découvertes dans les Gour Lazib (Glib Zegdou), situés dans le Sahara d'Algérie occidentale, et au pied du mont Chambi, situé dans la partie occidentale de la Tunisie centrale. Les spécimens fossiles sont très abondants mais essentiellement constitués de restes dentaires (dents isolées, fragments de mâchoires supérieures et inférieures). Malgré leur nature fragmentaire, ces fossiles nous permettent d'avoir un nouvel aperçu de l'importante diversité, jusqu'alors insoupçonnée, des chiroptères d'Afrique du Nord à la fin de l'Éocène inférieurdébut de l'Éocène moyen. Dans ce papier, nous décrivons et comparons ces nouveaux fossiles, précisons leurs affinités systématiques et phylogénétiques, et analysons les implications macroévolutives et paléobiogéographiques relatives à l'émergence des chiroptères modernes.

\section{MATÉRIEL ET MÉTHODES}

\section{LOCALITÉS ET CONTEXTE BIOCHRONOLOGIQUE}

La totalité du matériel fossile étudiée ici provient de deux formations continentales éocènes d'Afrique du Nord: Glib Zegdou en Algérie et Chambi en Tunisie (Fig. 1).

Le Glib Zegdou est une butte-témoin appartenant au complexe des Gour lazib (HGL pour Hammada Gour Lazib) situé dans le Sahara d'Algérie occidentale (Hammada du Dra; Fig. 1). La Formation du Glib Zegdou est réputée pour sa faune très diversifiée de vertébrés terrestres et aquatiques comprenant des poissons osseux, des squamates,
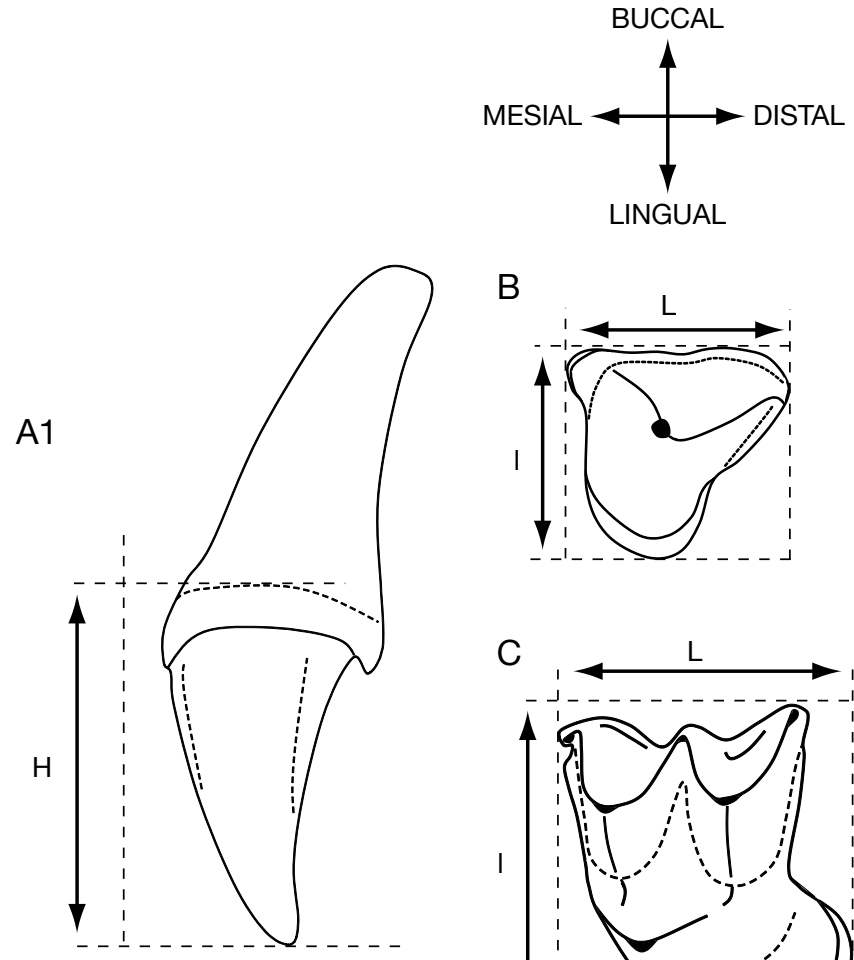

B
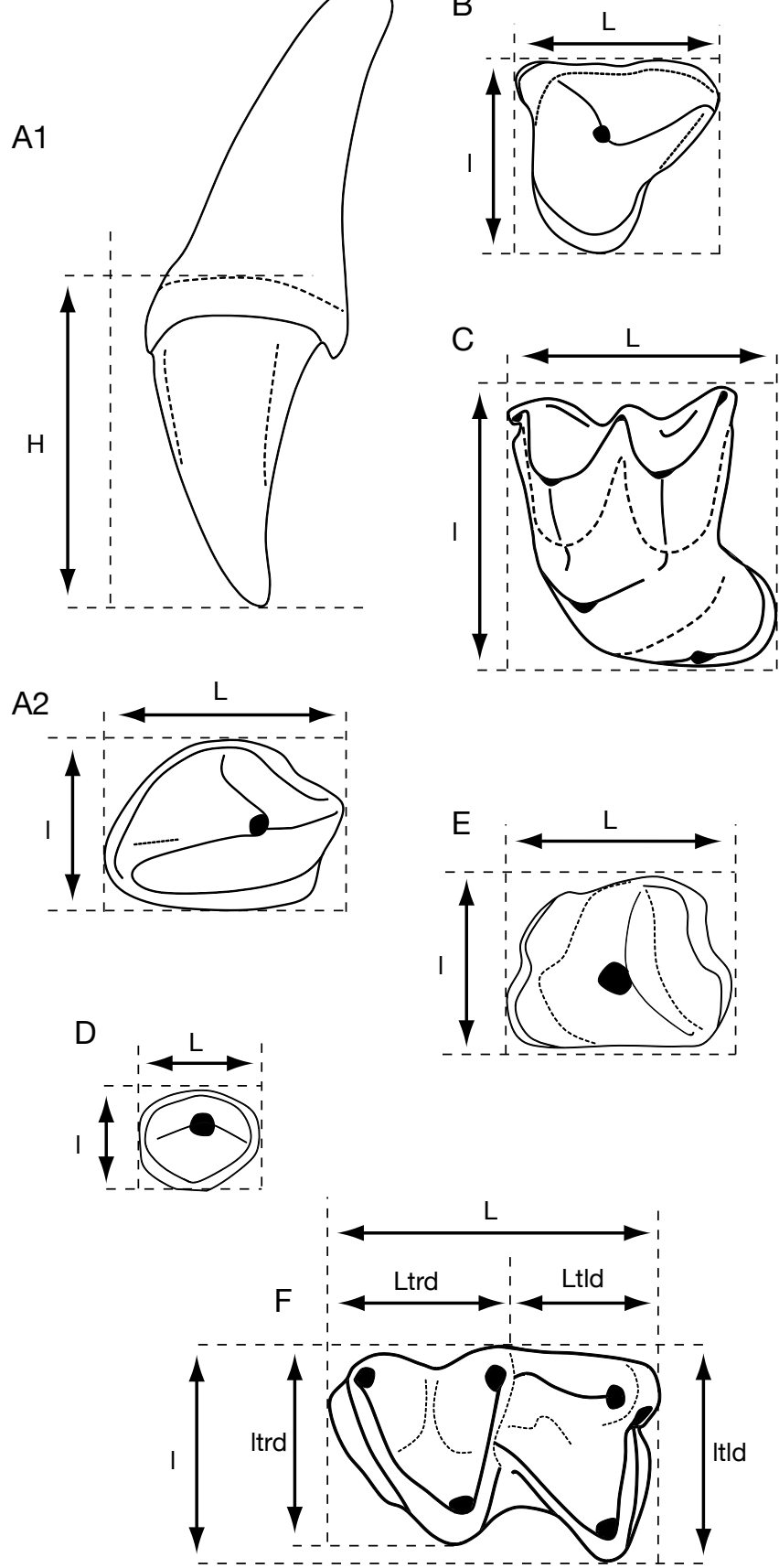

FIG. 3. - Mesures réalisées sur: A, canine supérieure; B, prémolaire supérieure; $\mathbf{C}$, molaire supérieure; $\mathbf{D}$, prémolaire inférieure antérieure, p2/3; $\mathbf{E}$, prémolaire inférieure postérieure, p4; F, molaire inférieure.

des chéloniens, des crocodiliens, des oiseaux ainsi que de nombreux mammifères (rongeurs, primates, créodontes, macroscélididés, hyracoïdes, possible "condylarthre», et chiroptères; Adaci et al. 2007; Tabuce et al. 2007, 2009a, 
b; Marivaux et al. 2011a, b; Mourer-Chauviré et al. 2011). Les chiroptères issus du Glib Zegdou proviennent exclusivement des niveaux HGL50 et HGL50' (Adaci et al. 2007).

Le Djebel Chambi (CBI) est situé dans la partie occidentale de la Tunisie centrale (région de Kasserine; Fig. 1). Les dépôts lacustres fossilifères constituent une couche bien distincte à la base de la formation continentale située au pied du versant Nord du djebel. Dans cette formation, une localité (CBI-1) a livré une faune et une flore bien diversifiées incluant des amphibiens et des squamates, un possible marsupial péradectidé (Crochet 1986) et plusieurs euthériens dont un possible érinaceomorphe (Gheerbrant \& Hartenberger 1999), des primates strepsirhiniens (Hartenberger \& Marandat 1992; Marivaux et al. 2013), un rongeur zegdoumyidé (Vianey-Liaud et al. 1994), un macroscélidé basal (Hartenberger 1986; Tabuce et al. 2007), et des hyracoïdes (Court \& Hartenberger 1992; Tabuce et al. 2011).

Les localités fossilifères de Chambi (CBI-1, CBI-2 et CBI-3) et du Glib Zegdou (HGL50 et HGL50') sont subcontemporaines et estimées à un âge compris entre l'Éocène inférieur terminal et l'Éocène moyen basal (Mebrouk et al. 1997, Adaci et al. 2007 ; Coster et al. 2012). Les deux sites ont livré de nombreux taxons communs comme la charophyte Raskyella sahariana Mebrouk, Mahboubi, Bessedik \& Feist, 1997 (Mebrouk et al. 1997), l'hyracoïde Microhyrax lavocati Sudre, 1979 (Tabuce et al. 2011) le primate Algeripithecus Godinot \& Mahboubi, 1992 (Tabuce et al. 2009b; Marivaux et al. 2013), le rongeur Zegdoumys Vianey-Liaud, Jaeger, Hartenberger \& Mahboubi, 1994 (Vianey-Liaud et al. 1994; Marivaux et al. 2011a) et le chiroptère Witwatia sigei Ravel, Marivaux, Tabuce, Ben Haj Ali, Essid \& Vianey-Liaud, 2012 (Ravel et al. 2012; Ravel et al. 2015). À cela s'ajoute l'hyracoïde Titanohyrax cf. tantulus et le macroscélidé ? Chambius sp., découverts dans les Gour Lazib (Adaci et al. 2007), qui sont deux taxons affines de formes décrites initialement à Chambi. Ces affinités taxinomiques suggèrent non seulement que les deux localités étaient proches dans le temps mais également qu'elles présentaient vraisemblablement des conditions paléoenvironnementales similaires (étude en cours).

\section{MATÉRIEL}

Les blocs de calcaire induré récoltés à CBI-1, CBI-2 et CBI-3 de Chambi ainsi que les grès à ciment carbonaté du niveau HGL50 et HGL50’ du Glib Zegdou ont été traités par attaques acides successives (acide acétique à $10 \%$ ), puis par lavage et tamisage. Plus de 500 spécimens de chiroptères ont été récoltés dans les deux localités (principalement à Chambi). Le matériel est essentiellement constitué de dents isolées et de quelques fragments de maxillaires et de dentaires. Seuls les spécimens les mieux préservés et les plus informatifs ont été inclus dans cette étude (289 spécimens). À Chambi, le nouveau locus 2 (CBI-2) a livré à lui seul $80 \%$ du matériel des trois localités. Les spécimens les mieux préservés ont été photographiés avec un Micro- scope Électronique à Balayage au Centre de Ressources en Imagerie Cellulaire de Montpellier (CRIC-M).

Le matériel de comparaison ayant servi aux études taxinomiques et systématiques est issu des collections de recherche de chiroptères fossiles et actuels hébergées à l'Institut des Sciences de l'Évolution de Montpellier (ISE-M), Université Montpellier (UM), réalisées par Bernard Sigé et François Catzeflis. Ce matériel de comparaison a été complété par de nombreux spécimens actuels et fossiles issus des collections ostéologiques Mammifères \& Oiseaux du Muséum national d'Histoire naturelle $(\mathrm{MNHN})$ à Paris.

\section{MORPHOLOGIE ET TERMINOLOGIE DES DENTS JUGALES}

La terminologie dentaire utilisée dans ce travail est inspirée de celles de Van Valen (1966) et Szalay (1969), basées sur le modèle tribosphénique classique (Fig. 2). La terminologie mandibulaire est également utilisée pour les spécimens documentés par des fragments de dentaire. Le protocole des prises de mesures est présenté sur la Figure 3. Différents schémas structuraux du talonide s'observent chez les microchiroptères. La reconnaissance de ces différents types se fait en fonction de la position relative des trois cuspides principales du talonide (i.e. entoconide, hypoconide et hypoconulide) ainsi que de leurs connexions avec la postcristide. Menu \& Sigé (1971) ont défini deux types bien distincts qui se retrouvent de manière récurrente chez les chiroptères insectivores et carnivores (Maitre 2014): - la "nyctalodontie» : elle se définit par la connexion directe de l'hypoconide et de l'hypoconulide via la postcristide; - la "myotodontie»: elle se définit par l'isolement de l'hypoconulide (réduit) se positionnant à la base distale de l'entoconide.

La «nyctalodontie» est plus fréquente que la «myotodontie» chez les chiroptères insectivores et se retrouve parmi toutes les familles actuelles. Dans le registre fossile, cette structure apparaît au début de l'Éocène inférieur (par ex. : Icaronycteris menui Russell, Louis \& Savage, 1973 et Archaeonycteris brailloni Russell, Louis \& Savage, 1973). Ce schéma constitue également le type le plus couramment observé au sein des taxons décris ici. La structure myotodonte est généralement observable chez les Vespertilionidae et les Noctilionidae. Dans le registre fossile, ce schéma apparaît comme une variation au sein des Vespertiliavus Schlosser, 1887 (Emballonuridae) et des Cuvierimops Legendre \& Sigé, 1982 (Molossidae) majoritairement nyctalodontes (Barghoorn 1977; Legendre 1980 ; Sigé 1990). Certaines dents, découvertes dans l'Éocène inférieur du Bassin de Paris, ont également développé la "myotodontie» (l'hypoconulide est toutefois légèrement déplacé buccalement; Russel et al.1973). Ces spécimens, associés dans un premier temps à l'espèce Icaronycteris menui par Russell et al. (1973), représente probablement une variation au sein d'un autre taxon, encore non identifié, à la morphologie dentaire plus dérivé (Smith et al. 2007).

La «nyctalodontie » et la «myotodontie» représentent deux extrêmes d'un ensemble comprenant de nombreux schémas structuraux intermédiaires. Ces derniers résultent souvent 
d'une variation au sein d'une petite population se définissant majoritairement par l'un des deux schémas structuraux précédemment mentionnés. Ainsi, la «sub-myotodontie» a été définie par Legendre (1984) chez les Molossidae. Elle se définit par la connexion de l'hypoconulide aux deux autres cuspides via une ramification de la postcristide. Ce schéma a été observé sur quelques spécimens d'une population qui présente en majorité la «nyctalodontie». La «sub-myotodontie» est souvent confondue avec la «myotodontie». La "sub-nyctalodontie" a été décrite par Ravel et al. (2011) et Sigé et al. (2012). Dans ce schéma, la postcristide fait la jonction directe avec l'hypoconulide déplacé buccalement par rapport à l'entoconide. L'hypoconulide (réduit) et l'entoconide sont reliés par une courte crête qui prolonge la postcristide. Cette variation de la "nyctalodontie» a été observée chez des chiroptères primitifs qui présentent généralement des dents nyctalodontes (par ex. : ?Archaeonycteris storchi Smith, Rana, Missiaen, Rose, Sahni, Singh \& Singh, 2007 et Hassianycteris kumari Smith, Rana, Missiaen, Rose, Sahni, Singh \& Singh, 2007).

Un dernier schéma structural, la "nécromantodontie", a été défini par Sigé et al. (2012) chez plusieurs lignées de l'Éocène inférieur; le talonide est ici caractérisé par un hypoconulide fort en position médiane et distale entre l'entoconide et l'hypoconide. Les trois cuspides sont reliées par le prolongement de la postcristide. Cet agencement n'est plus observable chez les chiroptères actuels. On le retrouve chez les formes fossiles telles que Necromantis (dont le nom du schéma est issu), Palaeophyllophora Revilliod, 1922, Honrovits Beard, Sigé \& Krishtalka, 1992, Ageina Russell, Louis \& Savage, 1973 ou Australonycteris Hand, Novacek, Godthelp \& Archer, 1994, et de manière plus sporadique chez Icaronycteris Jepsen, 1966, Archaeonycteris Revilliod, 1917 et Protonycteris Smith, Rana, Missiaen, Rose, Sahni, Singh \& Singh, 2007. Ce type de schéma structural n'est pas exclusif aux chiroptères mais se rapproche d'une morphologie généralisée tribosphénique que l'on retrouve également chez de nombreuses autres familles de mammifères (par ex. : Didelphidae Gray, 1821, Leptictidae Gill, 1872, Soricidae Fischer, 1814, Nyctitheriidae Simpson, 1928 et Adapisoriculidae van Valen, 1967; Sigé et al. 2012).

\section{ANALYSE CLADISTIQUE}

Plusieurs analyses cladistiques ont été réalisées. Les taxons et les caractères ont été choisis en fonction de la problématique soulevée. Compte tenu du type de matériel fossile considéré dans cette étude, les caractères ont été définis essentiellement sur les restes dentaires, d'autres sur les mandibules. Les matrices de caractères ont été construites avec le logiciel NDE (Nexus Data Editor v. 0.5.0; Page 2001). Les reconstructions phylogénétiques ont été réalisées avec le logiciel PAUP* v.4.0 beta 10 Win (Swofford 2002), selon une recherche exacte de l'arbre le plus parcimonieux («Branch and Bound», option [BandB]). La robustesse des nœuds a été évaluée en calculant l'indice de Bremer (Bremer 1994). Les matrices de caractères ainsi que les descriptions des caractères sont fournies avec les Annexes 1 et 2.

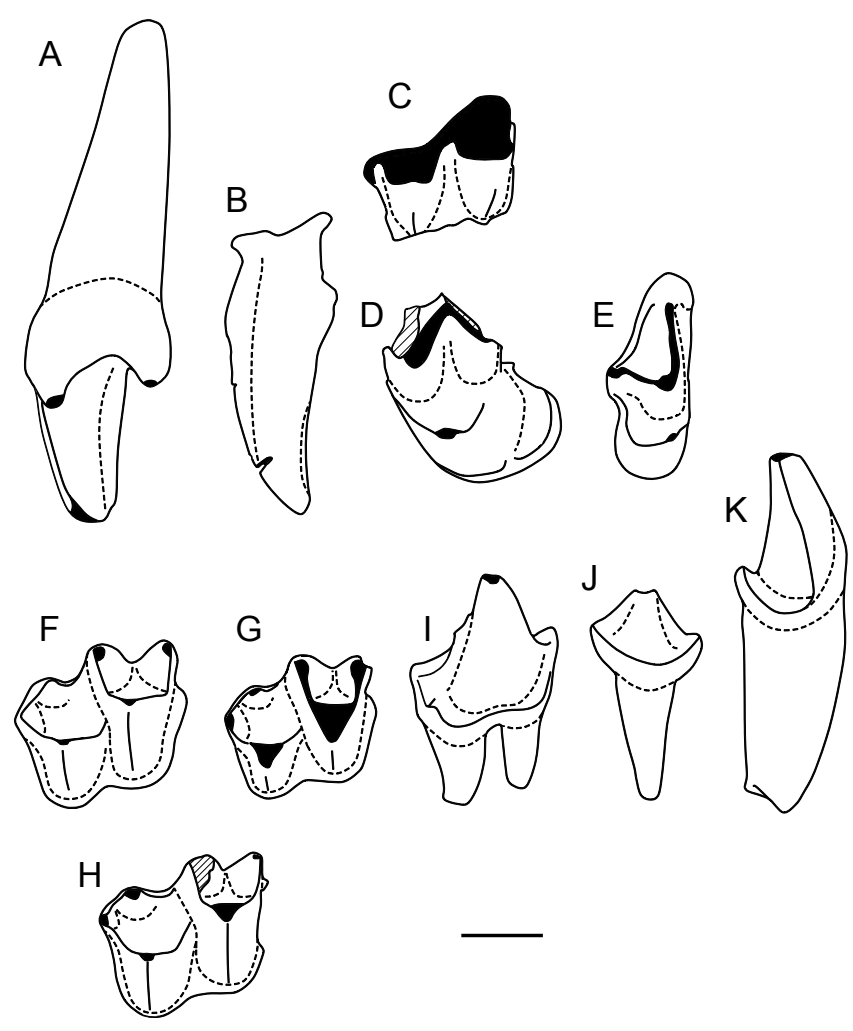

FIG. 4. - ?Necromantis fragmentum Ravel, n. sp. provenant des loci 1, 2 et 3 du Djebel Chambi dans la région de Kasserine, Tunisie: A, CBI-2-010, C1 droite en vue linguale; $\mathbf{B}, \mathrm{CBI}-1-243$, tubercule de $\mathrm{C} 1$ gauche en vue buccale; $\mathbf{C}, \mathrm{CBI}-2-$ 007, ectolophe de M1/2 gauche en vue occlusale; $\mathbf{D}, \mathrm{CBI}-2-008$, partie linguale de $\mathrm{M} 1 / 2$ gauche en vue occlusale; $\mathbf{E}, \mathrm{CBI}-2-009$, M3 droite en vue occlusale; F, CBI-1-244, $\mathrm{m} 1$ droite en vue occlusale; G, CBI-3-003, $\mathrm{m} 1$ droite en vue occlusale; H, CBI-2-011, m2 droite en vue occlusale; I, CBI-2-013, p4 droite en vue buccale; J, CBI-2-017, p2 gauche en vue buccale; K, CBI-2-016, c1 droite en vue buccale. Échelle: $1 \mathrm{~mm}$.

\section{SYSTÉMATIQUE}

Ordre CHIROPTERA Blumenbach, 1779 Sous-ordre MICROCHIROPTERA Dobson, 1875

Super-famille RHINOLOPHOIDEA Bell, 1836 Famille NeCROMANTIDAE Sigé, 2011

Genre ?Necromantis Weithofer, 1887

ESPÈCE TYPE. - Necromantis adichaster Weithofer, 1887 par désignation originale.

AUTRES ESPÈCES DU GENRE. - N. gezei Hand, Sigé \& Maitre, 2012; N. marandati Hand, Sigé \& Maitre, 2012.

?Necromantis fragmentum Ravel, n. sp. (Figs 4, 5; Tableau 1)

HOLOTYPE. - En l'absence de molaire complète, l'holotype considéré ici est la canine supérieure isolée droite CBI-2-010 marquant l'originalité de l'espèce nouvellement créée (Figs 4A; 5A).

Matériel EXAMINÉ. - CBI-1-243 (tubercule de C1 gauche; Figs 4B; 5B); CBI-2-007 (ectolophe de M1/2 gauche; Figs 4C ; 5C); CBI-2-008 
TABLEAU 1. - Mesures dentaires (en mm) de ?Necromantis fragmentum Ravel, $\mathrm{n}$. sp. provenant des loci 1, 2 et 3 du Djebel Chambi dans la région de Kasserine, Tunisie. Abréviations: L, longueur; I, largeur; H, hauteur; Ltdr, longueur du trigonide; Itdr, largeur du trigonide; Ltdl, longueur du talonide; Itdl, largeur du talonide. Le signe * derrière une valeur indique un biais dans la mesure causé par l'altération de la couronne.

\begin{tabular}{|c|c|c|c|c|c|c|c|c|c|c|}
\hline Spécimen & Localité & $\begin{array}{l}\text { Type de } \\
\text { matériel }\end{array}$ & $\mathbf{L}$ & $\mathbf{I}$ & $\mathbf{H}$ & Ltrd & Itrd & Ltld & Itld & Remarques \\
\hline CBI-1-243 & Chambi 1 & C1 gauche & $1,17^{*}$ & 1,04 & $3,15^{\star}$ & - & - & - & - & racine et base de la couronne manquantes \\
\hline CBI-2-010 & Chambi 2 & C1droite & 1,66 & 1,28 & $2,70^{*}$ & - & - & - & - & apex cassé \\
\hline CBI-2-007 & Chambi 2 & M1/2 gauche & 1,83 & 1,8 & - & - & - & - & - & uniquement l'ectolophe \\
\hline CBI-2-008 & Chambi 2 & M1/2 gauche & $2,19^{*}$ & $2,47^{*}$ & - & - & - & - & - & $\begin{array}{l}\text { partie linguale uniquement, } \\
\text { région du mésostyle conservée }\end{array}$ \\
\hline CBI-2-009 & Chambi 2 & M3 droite & 1,1 & 2,45 & - & - & - & - & - & - \\
\hline CBI-2-016 & Chambi 2 & c1 droite & 1,26 & 1,27 & $2,15^{\star}$ & - & - & - & - & partie apicale manquante \\
\hline CBI-2-021 & Chambi 2 & p2 droite & 1,36 & 0,73 & - & - & - & - & - & - \\
\hline CBI-2-023 & Chambi 2 & p2 droite & $1,30^{*}$ & 0,95 & - & - & - & - & - & extrémité distale cassée \\
\hline CBI-2-017 & Chambi 2 & p2 gauche & 1,43 & 0,91 & - & - & - & - & - & - \\
\hline CBI-2-018 & Chambi 2 & p2 gauche & 1,39 & 0,82 & - & - & - & - & - & - \\
\hline CBI-2-019 & Chambi 2 & p2 gauche & 1,31 & 0,93 & - & - & - & - & - & - \\
\hline CBI-2-020 & Chambi 2 & p2 gauche & 1,33 & 0,61 & - & - & - & - & - & - \\
\hline CBI-2-022 & Chambi 2 & p2 gauche & 1,69 & 0,75 & - & - & - & - & - & - \\
\hline CBI-2-013 & Chambi 2 & p4 droite & 1,85 & 1,01 & - & - & - & - & - & - \\
\hline CBI-2-015 & Chambi 2 & p4 droite & $1,76^{*}$ & 0,93 & - & - & - & - & - & - \\
\hline CBI-2-014 & Chambi 2 & p4 gauche & 1,75 & 0,99 & - & - & - & - & - & - \\
\hline CBI-1-244 & Chambi 1 & m1 droite & 2,24 & 1,4 & - & 1,36 & 1,3 & 1,09 & 1,4 & - \\
\hline CBI-3-003 & Chambi 3 & m1 droite & 2,02 & 1,27 & - & 1,17 & 1,26 & 0,84 & 1,28 & - \\
\hline CBI-2-011 & Chambi 2 & m2 droite & 2,26 & 1,38 & - & 1,18 & 1,33 & 1,08 & 1,38 & - \\
\hline CBI-2-012 & Chambi 2 & m1/2 droite & - & - & - & - & - & 1,12 & 1,41 & $\begin{array}{l}\text { trigonide à moitié cassé, seule une partie } \\
\text { du protoconide est conservée }\end{array}$ \\
\hline
\end{tabular}

(partie linguale d'une M1/2 gauche; Figs 4D; 5D); CBI-2-009 (M3 droite; Figs 4E; 5E) ; CBI-2-016 (c1 droite; Figs 4K; 5F); CBI-2-013 (p4 droite; Figs 4I ; 5I) ; CBI-2-014 (p4 gauche; Fig. 5J); CBI-2-017 (Figs 4J ; 5G), CBI-2-018 (Fig. 5H), CBI-2-019,CBI-2-020 et CBI-2022 (p2s gauches); CBI-2-021 et CBI-2-023 (p2s droites), CBI-1-244 (Figs 4F; 5K) et CBI-3-003 (m1s droites Figs 4G; 5L); CBI-2-011 (m2 droite; Figs 4H; 5M); CBI-2-012 (m1/2 droite; Fig. 5N).

ÉTYMOLOGIE. - Du latin «fragmentum» qui veut dire fragment ou débris, faisant référence au caractère fragmentaire du matériel attribué à ce taxon.

LOCALITÉ TYPE ET ÂGE. - Chambi (CBI) loci 1, 2 et 3, Éocène inférieur terminal-Éocène moyen basal, Djebel Chambi, situé dans la région de Kasserine, Tunisie.

DiAGNOSE. - Chiroptère avec une denture robuste, canine supérieure munie de deux tubercules secondaires puissants, une molaire supérieure avec un léger ectoflexus et un bord buccal très incliné mésiolingualement, une p4 présentant une ébauche de paraconide et un bassin du talonide plus développé.

\section{DESCRIPTION}

Ce taxon est représenté par les plus grands spécimens provenant des loci 2 et 3 de Chambi, et le deuxième plus grand du locus 1 après l'espèce Witwatia sigei Ravel, Marivaux, Tabuce, Ben Haj Ali, Essid \& Vianey-Liaud, 2012 (Ravel et al. 2012, 2015; Tableau 1).

Les canines supérieures CBI-2-010 et CBI-1-243 (Figs 4A, $\mathrm{B} ; 5 \mathrm{~A}, \mathrm{~B})$ sont attribuées à cette espèce en raison de leur taille importante et leur robustesse, compatibles avec les dents jugales associées. CBI-2-010 est très massive. La couronne est composée de trois tubercules: un principal en position mésiobuccale et deux secondaires assez forts situés aux bases mésio- et distolinguale du tubercule principal. Le tubercule principal est muni de deux faces: une face buccale convexe s'opposant à une face linguale quasi plate. Mésialement à la face buccale, un large sillon est visible. Le cingulum épais fait la liaison entre les deux cuspides secondaires lingualement. Il est plus fin et discontinu dans sa partie buccale. CBI-1-243 est une partie apicale du tubercule principal d'une canine (Figs 4B; 5B). Le tubercule est modérément recourbé vers la partie distale au niveau de l'apex. La face mésiobuccale est bombée, et porte un sillon s'étalant sur les trois quarts de la hauteur du tubercule comme sur CBI-2010 (Figs 4A; 5A). La face linguale, plate, est délimitée par une crête mésiale et une crête distale tranchantes.

CBI-2-007 est un fragment de dent correspondant à un ectolophe (Figs 4C; 5C). Son bord buccal est très incliné mésiolingualement au niveau du métacône puis s'aplanit au niveau du paracône. Le parastyle forme un "crochet» très recourbé vers l'extrémité mésiale. Il se positionne plus lingualement que le mésostyle et le métastyle. Le mésostyle, en partie effacé par l'usure de l'émail, est peu développé et en retrait par rapport au bord buccal. Le métastyle, en partie cassé, continue buccalement la postmétacrête de façon rectiligne. L'ectolophe forme un "W" légèrement comprimé dans sa partie distale ( $V$ » mésial plus ouvert que le « $V$ » distal). Le paracône se positionne plus lingualement et apparaît plus comprimé buccolingualement par rapport au métacône. Les deux tubercules buccaux sont espacés par une longue et large protofosse qui s'étend jusqu'à la base du mésostyle. Le précingulum est modérément large et s'oppose au postcingulum qui est beaucoup plus étroit.

Le spécimen CBI-2-008 est une moitié linguale de M1/2, dont une partie de la région du mésostyle et les flancs linguaux du paracône et du métacône ont été conservés (Figs 4D; 5D). Comme il a été observé sur l'ectolophe CBI-2-007, le 


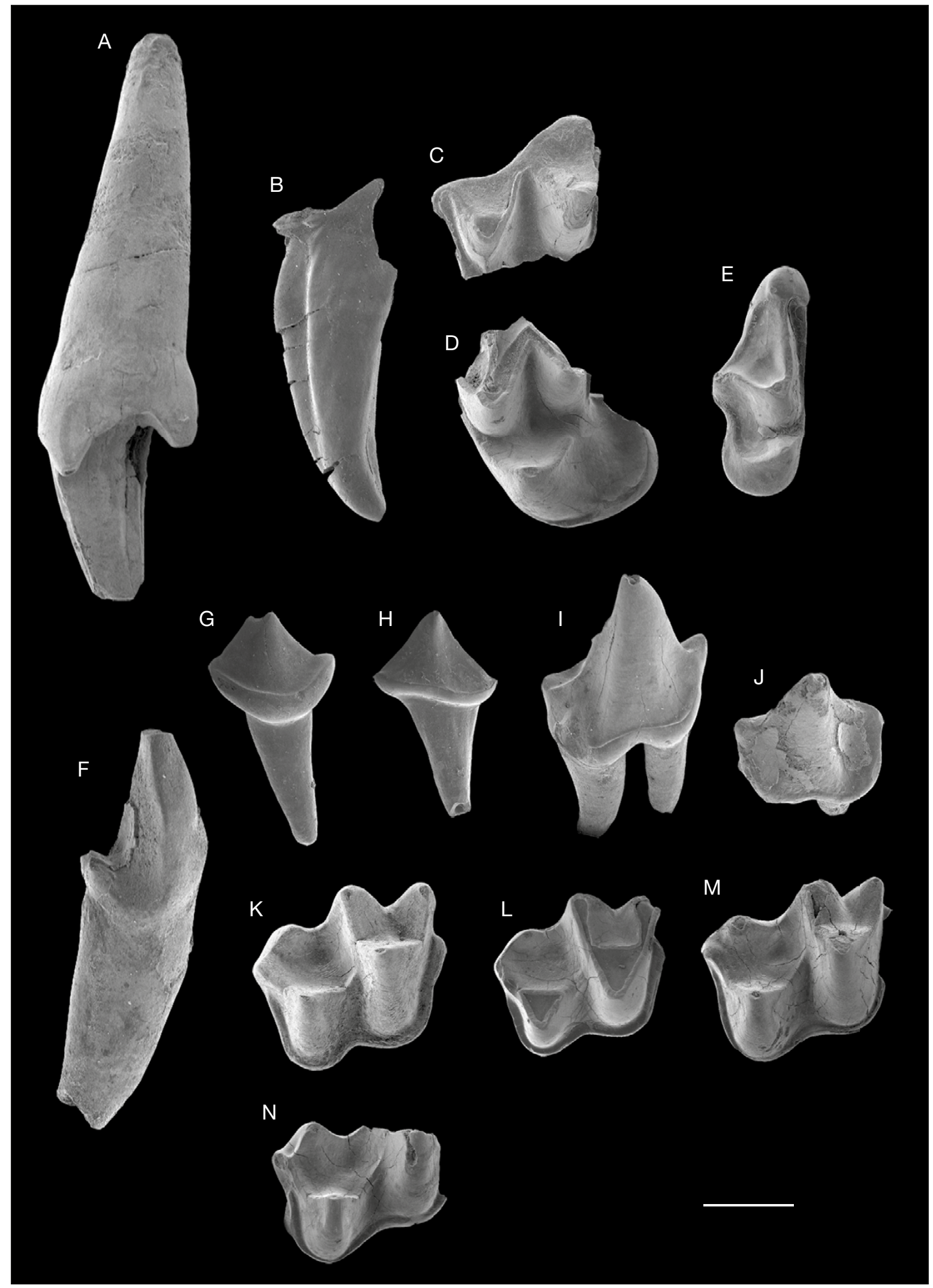

FIG. 5. - ?Necromantis fragmentum Ravel, $\mathrm{n}$. sp. provenant des loci 1, 2 et 3 du Djebel Chambi dans la région de Kasserine, Tunisie: A, CBI-2-010, C1 droite en vue linguale; $\mathbf{B}, \mathrm{CBI}-1-243$, tubercule de $\mathrm{C} 1$ gauche en vue buccale; C, CBI-2-007, ectolophe de M1-2 gauche en vue occlusale; $\mathbf{D}$, CBI-2-008, partie linguale de M1-2 gauche en vue occlusale; $\mathbf{E}, \mathrm{CBI}-2-009$, M3 droite en vue occlusale; $\mathbf{F}, \mathrm{CBI}-2-016$, c1droite en vue buccale; G, CBI-2-017, p2 gauche en vue buccale; $\mathbf{H}, \mathrm{CBI}-2-018$, p2 gauche en vue linguale; I, CBI-2-013, p4 droite en vue buccale; J, CBI-2-014, p4 gauche en vue semi-occlusale; K, CBI-1-244, m1 droite en vue occlusale; L, CBI-3-003, m1 droite en vue occlusale; M, CBI-2-011, m2 droite en vue occlusale; N, CBI-2-012, m1-2 droite en vue occlusale. Échelle: 1 mm. 
paracône est plus lingual que le métacône. Les deux tubercules sont néanmoins plus rapprochés limitant l'extension longitudinale de la protofosse. Le mésostyle forme un angle obtus en retrait du bord buccal. Le protocône est droit, haut et très pointu. La préprotocrête rectiligne remonte buccalement depuis le sommet du protocône pour rejoindre le mince précingulum. La postprotocrête s'oriente distobuccalement de façon symétrique à la préprotocrête. Elle se termine lingualement par rapport à la base du métacône laissant ainsi une ouverture distale à la protofosse. Le bassin du talon se projette distalement. Il s'étend vers la partie buccale jusqu'à la terminaison linguale du postcingulum. Le bassin du talon est ceinturé par un large cingulum lingual. Ce cingulum effectue un renflement distolingual au protocône sans pour autant réaliser un tubercule bien individualisé.

La M3, CBI-2-009 (Figs 4E; 5E), est complète est bien préservée. Cette dernière molaire supérieure apparaît plus réduite en largeur et en longueur que les deux autres spécimens. Son développement est néanmoins à tendance transversale. Le bord buccal de la dent est très incliné distolingualement et est muni d'un fort cingulum buccal continu. Le parastyle est particulièrement bien développé et prend la forme d'un large crochet projeté buccomésialement. Le paracône est réduit et déplacé vers le bord mésial. L'ectolophe, dépourvu de métacône, se termine distalement par un fragment de la prémétacrête. Le mésostyle conservé est peu développé et légèrement en retrait par rapport au bord buccal. Le protocône est faible et déjeté mésialement. Il se positionne ainsi sur le même axe buccolingual que le paracône. La préprotocrête se connecte mésiobuccalement au précingulum tandis que la postprotocrête se courbe distobuccalement pour rejoindre le postcingulum. Ce dernier, assez court, débute buccalement à la base de l'extrémité distale de la prémétacrête. La bordure linguale de la M3 est arrondie et dépourvue de cingulum.

La c1 (CBI-2-016; Figs $4 \mathrm{~K}$; 5F) possède un unique tubercule droit décomposé en une face mésiobuccale convexe et une face distolinguale plane. Les deux faces sont délimitées par une crête mésiale et une crête distale qui s'étendent depuis l'apex du tubercule. Le cingulum visible sur tout le pourtour de la couronne forme un bourrelet distolingual qui délimite un bassin étroit situé à la base de la cuspide.

CBI-2-017 à CBI-2-018 sont des dents possédant des couronnes très simples, unicuspidées, et dont la morphologie rappelle celle de p2 (Fig. 5G, H). Ces prémolaires ont une couronne ovale en vue occlusale. La cuspide se décompose en deux faces (une face buccale convexe s'opposant à une face linguale sub-plate) délimitées mésialement et distalement par des crêtes sagittales. La crête mésiale est légèrement plus courte et tranchante que la crête distale. La couronne est cernée par un épais cingulide continu, courbé buccalement et rectiligne lingualement.

Les p4s identifiées (CBI-2-013 et CBI-2-014; Figs 4I; $5 \mathrm{I}, \mathrm{J}$ ) sont biradiculées et possèdent une couronne allongée mésiodistalement (cependant moins longue que les m1-2s) et très étroite transversalement. La cuspide principale (i.e., protoconide) est haute et robuste. En position mésiale, le paraconide est légèrement plus lingual que le protoconide. Il s'élève au quart de la hauteur du protoconide. Les deux cuspides (protoconide et paraconide) sont connectées par une crête ayant une orientation à prédominance mésiale depuis l'apex du protoconide. À l'opposé, une large crête s'étend distolingualement depuis la pointe du protoconide. Elle effectue un renflement à mi-hauteur de la cuspide principale (comme un vestige de métaconide). La partie distale de la couronne est composée d'un talonide vestigial large et très projeté distalement. Le talonide se résume en un bassin traversé par une courte crête qui s'étend mésiodistalement depuis la base du protoconide jusqu'à l'extrémité distale de la couronne. Le bassin est circonscrit par un large cingulide qui s'étend jusqu'à la base mésiobuccale du protoconide.

Le trigonide des $\mathrm{m} 1-2 \mathrm{~s}$ est très massif et domine le talonide (Figs $4 ; 5$ ). Le paraconide est de taille quasi équivalente au métaconide. La projection mésiale du paraconide est plus prononcée sur $\mathrm{m} 1$ que sur $\mathrm{m} 2$, formant ainsi un trigonide plus ouvert sur cette dernière. Ceci est l'unique différence significative entre les deux types de molaires. Le protoconide est la cuspide dominante des molaires inférieures en termes de hauteur et de volume. Le talonide est de largeur et longueur comparables au trigonide. L'hypoconide se situe sur le même axe mésiodistal que le protoconide. La forte différence de hauteur entre l'hypoconide et le protoconide est associée à une flexion très prononcée du bord buccal de la couronne. La crête oblique, relativement courte, remonte depuis l'hypoconide jusqu'au niveau de la jonction entre la métacristide et la postprotocristide. L'entoconide, peu développé, se situe sur le même axe linguobuccal que l'hypoconide. L'hypoconulide, légèrement plus réduit que l'entoconide, occupe une position médiane et très distale par rapport à l'entoconide et à l'hypoconide. Il est relié à l'hypoconide et à l'entoconide par la postcristide. La position de l'hypoconulide et l'extension de la postcristide est caractéristique d'une structure nécromantodonte. La position de l'hypoconulide est plus linguale sur CBI-2-011 et CBI-2-012, soulignant ainsi une certaine variabilité de la structure du talonide au sein de l'espèce. Sur CBI-2-011, la crête reliant l'hypoconulide et l'entoconide n'apparaît pas, rappelant ainsi un aspect plus nyctalodonte. Le cingulide est continu et épais sur toutes les bordures mésiale, buccale et distale de la couronne.

\section{COMPARAISON ET DISCUSSION}

L'aspect robuste des dents de ?Necromantis fragmentum Ravel, n. sp. ainsi que la présence d'une p4 non molariforme sont des caractéristiques compatibles avec la diagnose des Necromantidae proposée par Sigé (2011a). La famille regroupe les genres Cryptobune Sigé, 2011et Necromantis sur la base de caractères soulignant un aspect carnassier. Cryptobune est documenté par un seul spécimen de très grande taille (un dentaire préservant $\mathrm{p} 4$ et $\mathrm{m} 1-3$ ) provenant d'une localité inconnue du Quercy (Sigé 2011a). L'étude de la morphologie dentaire de ce spécimen a conduit Sigé 
(2011a) à créer la famille des Necromantidae incluant également le genre Necromantis. ?Necromantis fragmentum Ravel, n. sp. de Chambi s'écarte de Cryptobune par sa taille beaucoup plus réduite, le trigonide moins compressé buccolingualement, des cuspides mieux individualisées et hautes, et le développement plus important du talonide des molaires inférieures et de $\mathrm{p} 4$.

Le genre Necromantis (espèce type $N$. adichaster) fut créé par Weithofer en 1887 sur la base d'un matériel très fragmentaire provenant de différentes poches karstiques datées du Paléogène du Quercy. Les spécimens représentatifs de ce taxon sont les plus grands parmi les faunes de chiroptères très diversifiées du Quercy. L'étude d'un nouveau matériel issu des anciennes et nouvelles collections ont permis d'identifier le genre Necromantis depuis l'Éocène moyen (Cuzal, MP13) jusqu'à l'Éocène supérieur (Rosière 5, MP 17b) regroupant plusieurs espèces ( $N$. adichaster, $N$. marandati et $N$. gezei; Weithofer 1887; Hand et al. 2012; Maitre 2014). Malgré le caractère très fragmentaire du taxon du Djebel Chambi, il est possible d'identifier de nombreuses affinités avec le genre Necromantis européen :

- C1 massive et recourbée distalement;

- un seul ectoflexus;

- mesostyle peu développé et légèrement en retrait du bord buccal;

- bord buccal nettement moins long que le bord lingual;

- postprotocrête relativement courte;

- protofosse ouverte distalement (semblable à $N$. adichaster,

les deux autres espèces ont une protofosse complètement fermée) ;

- extension du bassin du talon de la molaire supérieure;

- cingulum lingual arrondi et non cuspidé;

- M3 transverse;

- ectolophe de M3 dépourvu de métacône et de métacrête;

- protocône réduit de M3 et projeté mésialement (positionné sur le même axe buccolingual que le métacône);

- p4 allongée et étroite;

- trigonide massif et plus haut que le talonide, mais de

longueur et de largeur équivalentes à celles du talonide;

- paraconide et métaconide de taille similaire;

- entoconide nettement inférieur au métaconide;

- hypoconide moins volumineux que le protoconide et

légèrement plus buccal;

- molaires inférieures nécromantodontes.

Cependant les fossiles tunisiens présentent également des différences marquées en comparaison des formes européennes:

- C1 muni de deux fortes cuspides secondaires;

- bord buccal incliné mésiolingualement (cependant assez semblable au spécimen PRRR-70 du Quercy; Maitre 2014: pl. $25 \mathrm{f}$ et Hand et al. 2012: fig. 6.10D);

- ectoflexus nettement moins prononcé;

- cingulum lingual s'étendant plus mésialement à la base du protocône;

- M3 relativement plus longue;

- présence d'un paraconide sur $\mathrm{p} 4$;

- bassin du talonide de p4 plus développé;

- talonide légèrement plus étendu.
L'absence de rangées dentaires complètes ne permet pas d'aller plus en avant dans la comparaison. Les nombreuses similitudes observées entre les spécimens fossiles de Tunisie et Necromantis suggèrent néanmoins une étroite affiliation entre ces taxons et leur appartenance au même genre. Les divergences morphologiques moins nombreuses soulignent toutefois l'originalité du taxon tunisien notamment au niveau de la $\mathrm{C} 1$ qui porte deux cuspides secondaires. Le talonide légèrement plus développé et la présence d'un métaconide sur la p4 confèrent à ? $N$. fragmentum Ravel, n. sp. un aspect archaïque. Maitre (2014) a identifié deux lignées parmi cet ensemble générique. Ainsi la spéciation de $N$. adichaster à partir de $N$. marandati se traduit par un raccourcissement de la postprotocrête et une ouverture distale de la protofosse. Cette hypothèse est remise en question par le spécimen CBI-2-008, qui malgré son ancienneté vis-à-vis des deux taxons susnommés présente également une protofosse ouverte distalement.

Le genre Necromantis fut tout d'abord rapproché des Phyllostomidae Gray, 1825 et plus précisément des Phyllostominae (par ex. : Trachops fuliginosus Gray, 1847, Chrotopterus auritus Peters, 1856) par Weithofer (1887) sur les bases de la présence d'une $\mathrm{p} 3$ résiduelle déjetée vers le bord lingual, d'une réduction du talonide des $\mathrm{m} 2 \mathrm{~s}$ et $\mathrm{m} 3 \mathrm{~s}$, et de la réduction du nombre d'incisives inférieures (au nombre de deux sur chaque mandibule). Cependant comme le souligne Winge (1893) et Leche (1911), ces caractères sont également partagés avec d'autres familles actuelles (par ex. : Hipposideridae, Rhinolophidae, Megadermatidae). ?Necromantis fragmentum Ravel, n. sp. diffère des Phylostominae en ayant:

- une canine supérieure tri-tuberculée;

- un ectoflexus simple et peu prononcé;

- une forte inclinaison du bord buccal de la molaire supérieure;

- un mésostyle en retrait du bord buccal;

- une p4 allongée pourvue d'un métaconide;

- un cingulum lingual plus étendu mésialement;

- une courte postprotocrête ne se connectant à aucune autre structure.

Ces différences très marquées s'ajoutant à celle que l'on trouve entre les autres espèces mieux documentées de Necromantis et les Phyllostomidae, écartent toute affiliation possible entre le fossile tunisien et le groupe sud-américain actuel.

Sur la base d'une argumentation plus poussée et d'un matériel plus fourni, Revilliod (1920) a montré les similitudes entre Necromantis et les Megadermatidae, et plus particulièrement l'espèce Macroderma gigas. ?Necromantis fragmentum Ravel, n. sp., comme les autres espèces du même genre, partage avec les Megadermatidae: un mésostyle en retrait du bord buccal, la protofosse étroite mais ouverte distalement, un talon très projeté distalement constitué d'un large bassin, une M3 réduite et étirée transversalement, une p4 allongée, un trigonide puissant et une forte réduction de l'entoconide. En plus de ces similitudes observées, la canine CBI-2-010 attribuée à ?N. fragmentum Ravel, n. sp. a la particularité de posséder deux tubercules secondaires. 
TABLEAU 2. - Mesures dentaires (en mm) de ?Palaeophyllophora tunisiensis Ravel, $\mathrm{n}$. sp. provenant des loci 1 et 2 du Djebel Chambi dans la région de Kasserine, Tunisie. Abréviations: L, longueur; I, largeur; H, hauteur; Ltdr, longueur du trigonide; Itdr, largeur du trigonide; Ltdl, longueur du talonide; Itdl, largeur du talonide. Le signe * derrière une valeur indique un biais dans la mesure causé par l'altération de la couronne.

\begin{tabular}{|c|c|c|c|c|c|c|c|c|c|c|}
\hline Spécimen & Localité & $\begin{array}{l}\text { Type de } \\
\text { matériel }\end{array}$ & $\mathbf{L}$ & $\mathbf{I}$ & $\mathbf{H}$ & Ltrd & Itrd & Ltld & Itld & Remarques \\
\hline CBI-2-025 & Chambi 2 & C1 droite & 2,04 & 1,4 & $3,09^{*}$ & - & - & - & - & apex cassé \\
\hline CBI-1-245 & Chambi 1 & M1 droite & 2,13 & 2,45 & - & - & - & - & - & - \\
\hline $\mathrm{CBI}-1-246$ & Chambi 1 & M2 droite & $1,67^{*}$ & 2,46 & - & - & - & - & - & $\begin{array}{l}\text { couronne érodée, partie distale très altérée, } \\
\text { métastyle manquant et talon cassé }\end{array}$ \\
\hline CBI-2-024 & Chambi 2 & M2 gauche & $1,69^{*}$ & 2,49 & - & - & - & - & - & $\begin{array}{l}\text { couronne très érodée, structures } \\
\text { émoussées }\end{array}$ \\
\hline CBI-2-038 & Chambi 2 & c1 droite & 1,41 & 1,25 & - & - & - & - & - & apex du tubercule cassé \\
\hline CBI-2-028 & Chambi 2 & p2 droite & 1,12 & 0,79 & - & - & - & - & - & - \\
\hline CBI-2-027 & Chambi 2 & p2 gauche & 1,13 & 0,9 & - & - & - & - & - & - \\
\hline CBI-2-026 & Chambi 2 & p4 droite & 1,35 & 0,81 & - & - & - & - & - & - \\
\hline CBI-2-035 & Chambi 2 & $\mathrm{~m} 1 / 2$ droite & 2,02 & 1,11 & - & 1,11 & 1,11 & 0,91 & 1,11 & entoconide cassé \\
\hline CBI-2-036 & Chambi 2 & $\mathrm{~m} 1 / 2$ droite & - & - & - & 1,16 & 1,36 & - & - & trigonide seulement \\
\hline CBI-2-037 & Chambi 2 & m1/2 droite & - & - & - & - & - & 0,94 & 1,39 & talonide seulement \\
\hline CBI-2-029 & Chambi 2 & m1/2 gauche & 1,85 & 1,16 & - & 1,1 & 1,06 & 0,75 & 1,16 & - \\
\hline CBI-2-030 & Chambi 2 & $\mathrm{~m} 1 / 2$ gauche & 1,83 & 0,97 & - & 1,15 & 0,93 & 0,69 & 0,97 & - \\
\hline CBI-2-031 & Chambi 2 & $\mathrm{~m} 1 / 2$ gauche & 1,74 & 1 & - & 1,06 & 0,95 & 0,69 & 1 & - \\
\hline CBI-2-032 & Chambi 2 & $\mathrm{~m} 1 / 2$ gauche & 1,83 & 1,02 & - & 1,17 & 0,98 & 0,66 & 1,02 & - \\
\hline CBI-2-034 & Chambi 2 & $\mathrm{~m} 1 / 2$ gauche & 2,03 & 1,15 & - & 1,15 & 1,13 & 0,9 & 1,15 & couronne très érodée \\
\hline CBI-2-033 & Chambi 2 & m3 gauche & 1,52 & 0,83 & - & 0,95 & 0,83 & 0,55 & 0,5 & $\begin{array}{l}\text { dent encore enracinée dans un petit } \\
\text { fragment de dentaire }\end{array}$ \\
\hline
\end{tabular}

Cette configuration est partagée avec les Megadermatidae qui possèdent également deux tubercules secondaires (un très fort en position distale du tubercule principal et le second en position mésiolinguale, formé par le cingulum). L'attribution au genre Necromantis de la canine supérieure de Chambi, si elle venait à être confirmée, indiquerait que Necromantis et les Megadermatidae partagent un ancêtre commun. Toutefois, Necromantis ne possèdent pas les caractéristiques dentaires qui définissent le groupe des Megadermatidae, qui sont:

- ectolophe ayant une forte action tranchante: le paracône et le métacône sont peu développés et sont très rapprochés l'un de l'autre;

- postmétacrête deux fois plus longue que la préparacrête et projetée distolingualement (fonction spécialisée de cisaillement);

- protocône très réduit;

- postcingulum absent;

- P4 dépourvu de cuspides secondaires;

- talonide des molaires inférieures réduit par rapport au trigonide;

- trigonide de $\mathrm{m} 1$ très ouvert avec un paraconide très déjeté mésialement.

De plus l'espèce provenant de Chambi s'écarte des Megadermatidae par:

- le tubercule mésiolingual de $\mathrm{C} 1$ plus développé;

- le tubercule distolingual de C1 déplacé lingualement (il est dans l'axe du tubercule principal sur les canines de Megadermatidae);

- le bord buccal fortement incliné mésiolingualement;

- le mésostyle moins développé.

Les différences énoncées ci-dessus s'ajoutent à celles relevées sur les crânes par Hand et al. (2012) entre les Mégadermatidae et Necromantis adichaster. L'accumulation de ces divergences ne permet pas d'inclure le genre Necromantis dans la famille des Megadermatidae, et conforte le statut familial des Necromantidae.
Famille HipPOSIDERIDAE Miller, 1907

Genre ?Palaeophyllophora Revilliod, 1917

ESPÈCE TYPE. - Palaeophyllophora oltina Delfortrie, 1873 par désignation subséquente (Revilliod 1922).

Autres ESPÈCES DU GENRE. - P. quercyi Revilliod, 1917 ; $P$. nova Maitre, 2014; P. parva Maitre, 2014; P. rosierensis Maitre, 2014. Deux autres espèces $(P$. sp. A et $\mathrm{B})$ sont référencées dans Maitre (2014).

?Palaeophyllophora tunisiensis Ravel, n. sp. (Figs 6, 7; Tableau 2)

Holotype. - Spécimen CBI-1-245, M1 droite, Figs 6B ; 7B.

Matériel eXaminé. - CBI-2-025 (C1 droite; Figs 6A; 7B); CBI-1-246 (M2 droite); CBI-2-024 (M2 gauche; Figs 6C; 7C); CBI-2-038 (c1 droite); CBI-2-028 (p2 droite); CBI-2-027 (p2 gauche; Figs 6D; 7D); CBI-2-026 (p4 droite); CBI-2-035 (m1/2 droite); CBI-2-029 (Figs 6E; 7E), CBI-2-030 (Figs 6F; 7F), CBI-2-031 (Fig. 7G), CBI-2-032 (Fig. 7H) et CBI-2-034 (m1/2s gauches); CBI-2-036 (trigonide de m1/2 droite); CBI-2-037 (talonide de $\mathrm{m} 1 / 2$ droite); CBI-2-033 (m3 gauche; Figs 6G ; 7I).

ÉTYMOLOGIE. - Le nom de l'espèce fait référence à la Tunisie.

LoCAlité TYPE ET ÂGE. - Chambi (CBI) loci 1 et 2, Éocène inférieur terminal-Éocène moyen basal, Djebel Chambi, situé dans la région de Kasserine, Tunisie.

DiAgNOSE. - Espèce caractérisée par l'absence de mésostyle et de postprotocrête sur M1-2. Le protocône de ces mêmes dents est très volumineux. Le cingulum linguodistal rejoint le postcingulum. Le talonide des m1-2 est bien développé, aussi long et large que le trigonide. Cuspides du talonide très espacées cernant un long, large et profond bassin. 


\section{DESCRIPTION}

CBI-2-025 est une canine supérieure massive, dont la cuspide principale est puissante (Figs 6A; 7B). La face linguale est plate, alors que la face buccale est convexe. Cette dernière est traversée par un sillon de direction mésiobuccale. Le cingulum est développé sur toute la circonférence de la cuspide, et forme deux épais bourrelets (mésio- et distolingual) à la base de la couronne. La partie buccale du cingulum est peu visible (en partie altérée par l'érosion).

Les molaires supérieures CBI-1-246 et CBI-2-024 sont altérées et présentent des structures fortement émoussées. La description des molaires supérieures se focalisera donc sur l'unique spécimen CBI-1-245, en meilleur état de préservation (Figs 6B; 7B). Les molaires supérieures sont sub-triangulaires en vue occlusale. Le bord buccal est beaucoup plus long que la partie linguale donnant cet aspect d'amincissement lingual. Le développement transversal est plus marqué chez CBI-2-024 et CBI-1-246 (Figs 6C; 7C). Le parastyle de ces deux dents est également moins projeté mésialement mais plus étiré buccalement que chez CBI-1-245. Ces différences semblent être les seuls critères marquants permettant de distinguer une M1 (CBI-1-245) d'une M2 (CBI-2-024 et CBI-1-246). L'ectolophe est très ouvert du fait des crêtes latérales (i.e., préparacrête et postmétacrête) très divergentes. Le mésostyle est absent, une condition singulière qui differe du patron dilambdodonte courant chez les chiroptères. Le cingulum buccal est assez épais. Il forme une muraille continue qui délimite buccalement une surface d'usure surplombant le paracône et le métacône. Le bord buccal est creusé par un unique ectoflexus très profond. La postparacrête et la prémétacrête sont très courtes et se rejoignent en profondeur pour former une échancrure entre le paracône et le métacône. La postmétacrête est très haute et tranchante. Elle se projette distobuccalement vers le métastyle. La préparacrête est plus courte et plus axée buccolingualement. Elle fait la jonction entre le métacône et le parastyle. Ce dernier est faiblement incurvé en direction de la partie mésiolinguale. Le métacône et le paracône sont très éloignés lingualement de la bordure buccale. Ces deux cuspides sont de taille équivalente. Le paracône est légèrement plus lingual que le métacône. Cette différence est légèrement plus marquée sur CBI-2-024 et CBI-1-246. Le protocône est la structure dominante de la partie linguale de la couronne. Il est conique, très volumineux et pointu. Il se positionne légèrement plus distalement que le paracône. La préprotocrête remonte mésiobuccalement et rejoint un large précingulum. La postprotocrête est en revanche très courte, et ne rejoint aucune autre structure, laissant ainsi une ouverture distale à la protofosse. Les flancs du paracône et du métacône jointifs ne permettent pas une extension buccale de la protofosse. Le cingulum lingual encercle la base du protocône depuis son flanc mésial jusqu’au postcingulum. Le cingulum lingual s'épaissit considérablement dans la région distolinguale pour former sur le talon un bourrelet de forme arrondi.

CBI-2-038 correspond à la base d'une couronne d'une canine inférieure très massive. La robustesse de cette dent semble correspondre avec la canine supérieure décrite ci-dessus (CBI-2-025). La cuspide a été sectionnée. Il est possible malgré tout d'identifier les deux faces du tubercule: une face linguale concave, connectée à un large bassin distolingual, et une face convexe buccale. Les deux faces sont délimitées par une crête distolinguale très fine et une crête distobuccale plus émoussée. Le cingulide est continu mais devient à peine visible dans sa partie la plus buccale.

CBI-2-026 représente une $\mathrm{p} 4$ droite. Malheureusement, l'émail de la prémolaire a été fortement altéré, ce qui ne permet pas de faire une description précise du spécimen. La couronne est prémolariforme et possède un contour rectangulaire qui s'amincit vers la partie mésiale (en vue occlusale). Seul le protoconide, dominant et puissant, est présent mais fortement érodé. Le talonide est dominé par un bassin dont l'extension est modérée.

De par la simplicité de la couronne, les spécimens CBI-2027 (Figs 6D; 7D) et CBI-2-028 peuvent être attribués ici à des p2s. Leur couronne, de contour ovale en vue occlusale, est munie d'une forte cuspide positionnée mésialement. Deux crêtes sagittales (une mésiale et une distale) s'étendent depuis le somment de cette cuspide et divisent la dent en deux parties (buccale et linguale) quasi symétriques. Un épais cingulide encercle de manière continue la base de la cuspide.

Parmi les molaires inférieures, il n'a pas été possible de distinguer de manière précise les $\mathrm{m} 1 \mathrm{~s}$ des $\mathrm{m} 2 \mathrm{~s}$, soit parce que l'un des deux morphotypes est absent, soit parce que la différence morphologique entre les deux types de dents est ténue. En vue occlusale, le trigonide de ces dents prend la forme d'un triangle quasi équilatéral. Le paraconide, incliné mésialement, est légèrement plus réduit que le métaconide et le protoconide. Le protoconide reste la cuspide la plus volumineuse de la dent. Le talonide est aussi long et aussi large que le trigonide. Cependant le talonide reste significativement moins élevé que le trigonide. L'espacement des cuspides du talonide se traduit par un vaste bassin, qui plus est très profond. L'hypoconide, pincé distomésialement, se situe plus mésialement par rapport à l'entoconide. La longue cristide oblique s'étend depuis l'apex de l'hypoconide jusqu'à la base de la muraille distale du trigonide. L'entoconide, très distal, est petit et peu pointu par rapport à l'hypoconide. L'hypoconulide, légèrement plus réduit que l'entoconide, occupe une position médiane et très distale par rapport à l'entoconide et à l'hypoconide. Il est relié à l'hypoconide et à l'entoconide par la postcristide. Une telle position de l'hypoconulide associée à l'extension de la postcristide, caractérisent une structure nécromantodonte (bien visible sur CBI-2-029; Figs 6E; 7E). Les autres molaires présentent un hypoconulide légèrement plus buccal et n'ont pas de crête reliant l'hypoconulide à l'entoconide (CB2-031 et CB2-032; Fig. 7G, H). Par ces traits, le talonide de ces dents serait plus d'aspect nyctalodonte. Le cingulide est continu et épais sur toutes les bordures mésiale, buccale et distale de la couronne. $\mathrm{La} \mathrm{m} 3$ est plus réduite que les $\mathrm{m} 1 \mathrm{~s}$ et $\mathrm{m} 2 \mathrm{~s}$. Le trigonide de $\mathrm{m} 3$ differe du trigonide des m1-2s en ayant une taille plus petite et une forte réduction du paracône et du métacône. Le talonide est également fortement réduit, allongé mais très étroit. Les cuspides associées au talonide ne sont pas identifiables à l'exception de l'hypoconide, lequel est très réduit et déplacé lingualement. Le cingulide de $\mathrm{m} 3$ est continu mais il s'amincit fortement au niveau de l'hypoconide. 

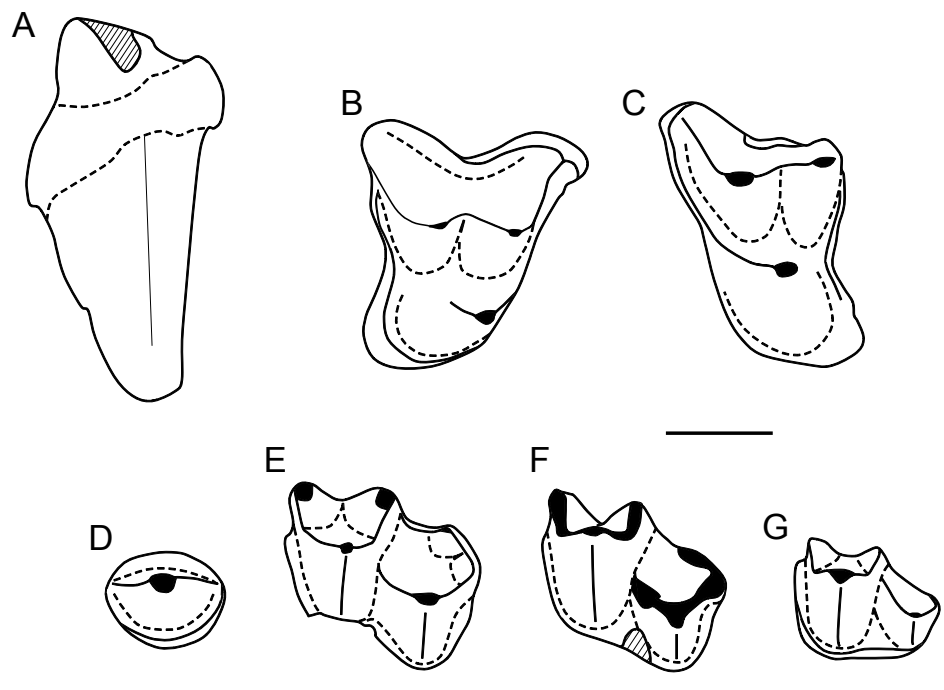

FIG. 6. - ?Palaeophyllophora tunisiensis Ravel, n. sp. provenant des loci 1 et 2 du Djebel Chambi dans la région de Kasserine, Tunisie: A, CBI-2-025, C1 droite en vue occlusale; B, CBI-1-245, M1 droite en vue occlusale; C, CBI-2-024, M2 gauche en vue occlusale; D, CBI-2-027, p2 gauche en vue occlusale; E, CBI-2029, m1/2 gauche en vue occlusale; F, CBI-2-030, m1/2 gauche en vue occlusale; G, CBI-2-033, m3 gauche en vue occlusale. Échelle: 1 mm.

\section{COMPARAISON ET DISCUSSION}

L'absence du patron dilambdodonte pour les molaires supérieures est peu fréquente chez les chiroptères. C'est pourquoi l'interprétation taxonomique de ces dents est difficile. Néanmoins, la simplicité structurale de ces molaires, lesquelles sont dépourvues de conule, la projection distolinguale du talon, une divergence des préparacrête et postmétacrête, un développement du cingulum (fort et arrondi), permet d'attribuer ce matériel à un chiroptère. De plus, sur les molaires inférieures associées, la présence d'un épais cingulide continu et le développement et la position de l'hypoconulide renforcent la signature chiroptérienne de ce taxon de Chambi. Plusieurs formes actuelles se caractérisent par une perte totale ou partielle du complexe en "W» de l'ectolophe:

- chez les Phyllostomidae, la plupart des formes ont totalement perdu la dilambdodontie, au profit d'une morphologie plus adaptée au régime frugivore, nectarivore ou hématophage. La forte modification du schéma insectivore chez ces Phyllostomidae ne permet pas d'identifier des similarités avec les fossiles tunisiens;

- au sein des Vespertilionidae, le genre Scotophilus présente une forte modification de l'ectolophe avec un mésostyle fortement diminué et nettement en retrait du bord buccal (parfois effacé par l'usure dentaire), un unique ectoflexus, une postparacrête et une prémétacrête très courtes, ainsi qu'une protofosse absente entre le paracône et le métacône. Malgré un agencement similaire de l'ectolophe, les fossiles tunisiens s'écartent du genre Scotophilus par la projection distolinguale du talon plus prononcée, l'ectolophe nettement plus ouvert, la protofosse ouverte distalement, le talonide plus développé longitudinalement et présentant une structure nécromantodonte.

Les molaires supérieures de ?Palaeophyllophora tunisiensis Ravel, n. sp. présentent un talon bien développé qui se projette distolingualement, un ectolophe ouvert et une protofosse réduite. Ces caractéristiques sont communes au sein du grand ensemble Rhinolophoidea. Parmi les rhinolophoïdes fossiles, Palaeophyllophora se défini par un ensemble de caractères dentaires dont certains se retrouvent sur les spécimens tunisiens. Le genre Paleophyllophora est attesté dans de nombreuses localités du Quercy depuis le milieu de l'Éocène moyen (MP13, St Maximin) jusqu'à l'Oligocène inférieur (MP23, La Gardiole 3; Revilliod 1917 ; Sigé 1978 ; Maitre 2014). Il représente un genre archaïque au sein des Hipposideridae qui se traduit notamment par la présence d'une p3 vestigiale (semblable aux Rhinolophidae). Les fossiles de Tunisie se rapprochent de cet ensemble Européen par :

- l'aspect robuste de la dentition;

- la présence d'un sillon mésiobuccal sur C1 ;

- un unique ectoflexus central ;

- les postparacrête et prémétacrête très courtes;

- une forte réduction et un retrait lingual du mésostyle;

- un paracône plus lingual que le métacône;

- l'absence d'extension buccale de la protofosse;

- le talonide des m1-2s légèrement plus court que le trigonide;

- des molaires inférieures nécromantodontes ou tout du moins présentant un hypoconulide buccalement déplacé par rapport à l'entoconide;

- la réduction du talonide de la m3.

Ces similitudes correspondent pour la plupart aux caractères diagnostiques du genre Palaeophyllophora (Revilliod 1922). Les spécimens étudiés ici présentent cependant de fortes divergences également avec les espèces de Palaeophyllophora européennes:

- le bassin lingual de C1 est absent;

- le cingulum buccal de C1 est peu prononcé;

- le mésostyle est absent sur M1-2 (absence du patron dilambdodonte typique);

- le cingulum lingual est très épais, formant un bourrelet projeté distolingualement (surtout sur M1); 


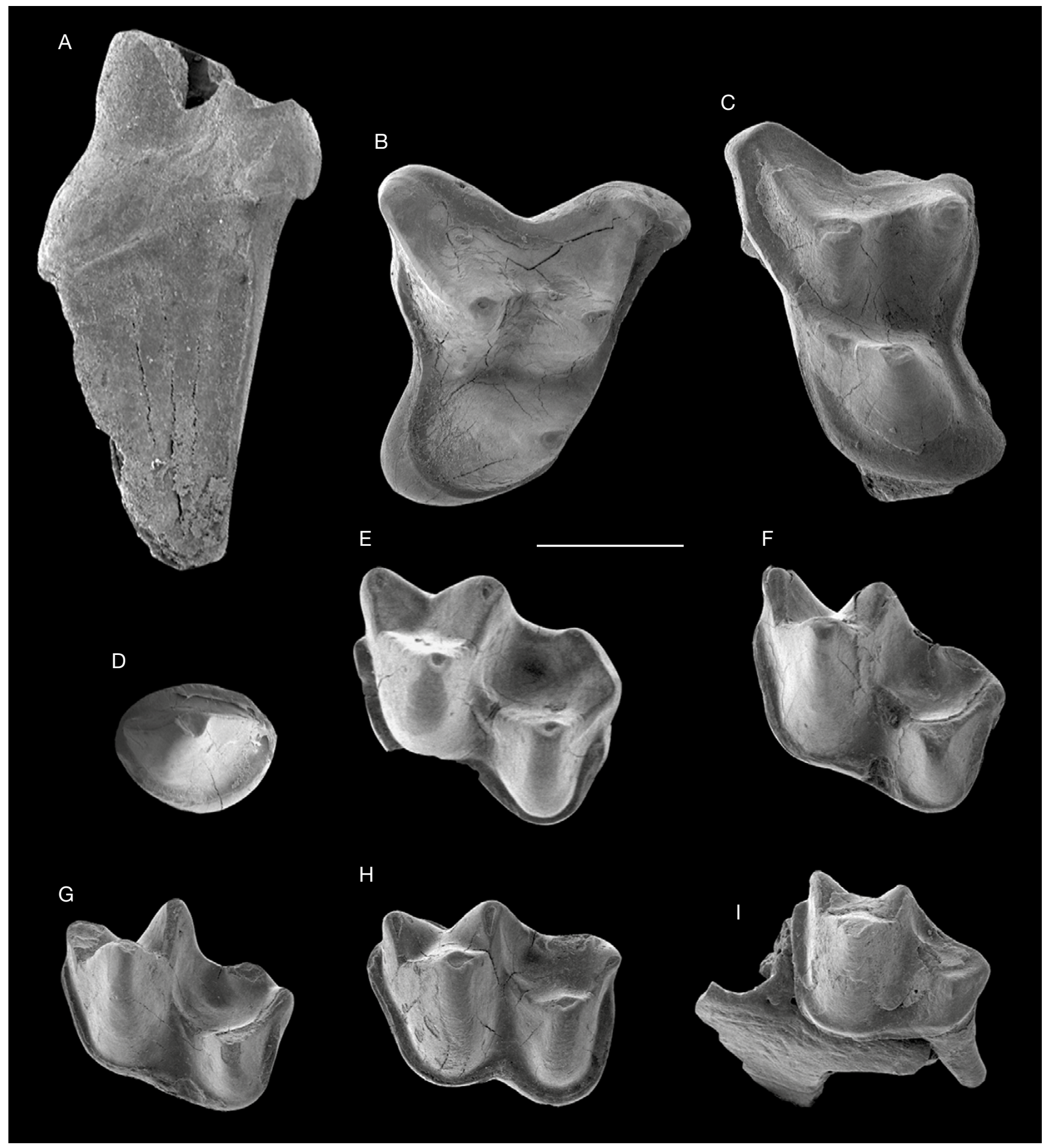

FIG. 7. - ?Palaeophyllophora tunisiensis Ravel, n. sp. provenant des loci 1 et 2 du Djebel Chambi dans la région de Kasserine, Tunisie: A, CBI-2-025, C1 droite en vue linguale; B, CBI-1-245, M1 droite en vue occlusale; $\mathbf{C}, \mathrm{CBI}-2-024, \mathrm{M} 2$ gauche en vue occlusale; $\mathbf{D}, \mathrm{CBI}-2-027$, p2 gauche en vue occlusale; $\mathbf{E}$, CBI-2029, m1/2 gauche en vue occlusale; $\mathbf{F}, \mathrm{CBI}-2-030, \mathrm{~m} 1 / 2$ gauche en vue occlusale; $\mathbf{G}, \mathrm{CBI}-2-031, \mathrm{~m} 1 / 2$ gauche en vue occlusale; $\mathbf{H}, \mathrm{CBI}-2-032, \mathrm{~m} 1 / 2$ gauche en vue occlusale; I, CBI-2-033, m3 gauche en vue occlusale. Échelle: $1 \mathrm{~mm}$.

- la présence d'une connexion entre le postcingulum et le cingulum lingual (structure continue);

- une très courte extension de la postprotocrête, laissant une ouverture distale à la protofosse;

- le protocône est moins massif mais relativement plus élevé;
- les molaires inférieures sont légèrement plus allongées;

- l'hypoconide occupe une position plus buccale.

La pauvreté du matériel ainsi que les divergences énoncées ci-dessus ne permettent pas d'assigner de façon formelle ces nouveaux fossiles des localités du Chambi au genre 
TABleau 3. - Mesures dentaires (en mm) de Hipposideros (Pseudorhinolophus) africanum Ravel, n. sp. provenant des loci 1, 2 et 3 du Djebel Chambi dans la région de Kasserine, Tunisie Abréviations: L, longueur; I, largeur; H, hauteur; Ltdr, longueur du trigonide; Itdr, largeur du trigonide; Ltdl, longueur du talonide; Itdl, largeur du talonide. Le signe * derrière une valeur indique un biais dans la mesure causé par l'altération de la couronne.

\begin{tabular}{|c|c|c|c|c|c|c|c|c|c|c|}
\hline Spécimen & Localité & Type de matériel & $\mathbf{L}$ & I & $\mathbf{H}$ & Ltrd & Itrd & Ltld & Itld & Remarques \\
\hline CBI-2-045 & Chambi 2 & C1 droite & 1,22 & 0,85 & 1,77 & - & - & - & - & - \\
\hline CBI-2-047 & Chambi 2 & C1 droite & 1,31 & 0,86 & 2,31 & - & - & - & - & - \\
\hline CBI-2-048 & Chambi 2 & C1 droite & 1,54 & 0,82 & 2,24 & - & - & - & - & - \\
\hline CBI-2-046 & Chambi 2 & C1 gauche & 1,18 & 0,8 & 1,77 & - & - & - & - & - \\
\hline CBI-2-049 & Chambi 2 & C1 gauche & 1,38 & 0,95 & 2,42 & - & - & - & - & - \\
\hline CBI-1-247 & Chambi 1 & $\begin{array}{l}\text { fragment de maxillaire } \\
\text { droit avec } \mathrm{M} 2 / \mathrm{M} 3\end{array}$ & $\begin{array}{l}1,55 / \\
0,82\end{array}$ & $\begin{array}{l}1,70 / \\
1,70\end{array}$ & - & - & - & - & - & - \\
\hline CBI-1-248 & Chambi 1 & M1 droite & 1,53 & 1,6 & - & - & - & - & - & - \\
\hline CBI-2-039 & Chambi 2 & M2 droite & 1,54 & 1,79 & - & - & - & - & - & - \\
\hline CBI-2-043 & Chambi 2 & M2 droite & 1,66 & 1,87 & - & - & - & - & - & - \\
\hline CBI-2-044 & Chambi 2 & M2 droite & 1,69 & 1,97 & - & - & - & - & - & - \\
\hline CBI-2-041 & Chambi 2 & M3 droite & 0,96 & $1,62^{*}$ & - & - & - & - & - & protocône cassé \\
\hline CBI-2-042 & Chambi 2 & M3 droite & 0,89 & 1,6 & - & - & - & - & - & - \\
\hline CBI-2-040 & Chambi 2 & M3 gauche & 0,86 & $1,74^{\star}$ & - & - & - & - & - & $\begin{array}{l}\text { bord lingual du protocône } \\
\text { cassé }\end{array}$ \\
\hline CBI-2-057 & Chambi 2 & c1 droite & 0,95 & 0,7 & 1,61 & - & - & - & - & - \\
\hline CBI-2-058 & Chambi 2 & c1 droite & 1 & 0,61 & 1,61 & - & - & - & - & - \\
\hline CBI-2-056 & Chambi 2 & c1 gauche & 1,14 & 0,79 & 1,67 & - & - & - & - & - \\
\hline CBI-2-054 & Chambi 2 & p2 droite & 0,92 & 0,64 & - & - & - & - & - & - \\
\hline CBI-2-055 & Chambi 2 & p2 droite & 0,91 & 0,63 & - & - & - & - & - & - \\
\hline CBI-3-004 & Chambi 3 & p2 gauche & 0,9 & 0,47 & - & - & - & - & - & - \\
\hline CBI-2-220 & Chambi 2 & p2 gauche & 0,99 & 0,65 & - & - & - & - & - & - \\
\hline \multirow[t]{2}{*}{ CBI-2-053 } & Chambi 2 & fragment de dentaire & $1,52 /$ & $0,87 /$ & - & $0,88 /$ & $0,84 /$ & $0,65 /$ & $0,85 /$ & - \\
\hline & & droit avec m2/m3 & 1,34 & 0,85 & & 0,81 & 0,85 & 0,58 & 0,55 & \\
\hline CBI-2-050 & Chambi 2 & $\mathrm{~m} 1 / 2$ droite & 1,26 & 0,84 & - & 0,89 & 0,83 & 0,56 & 0,84 & - \\
\hline CBI-2-051 & Chambi 2 & m1/2 droite & 1,39 & 0,82 & - & 0,82 & 0,8 & 0,57 & 0,82 & - \\
\hline CBI-1-249 & Chambi 1 & $\mathrm{~m} 1 / 2$ gauche & 1,32 & 0,84 & - & 0,78 & 0,77 & 0,54 & 0,84 & - \\
\hline CBI-1-250 & Chambi 1 & m1/2gauche & 1,3 & 0,81 & - & 0,78 & 0,74 & 0,51 & 0,81 & - \\
\hline CBI-2-052 & Chambi 2 & $\mathrm{~m} 1 / 2$ gauche & 1,35 & 0,87 & - & 0,74 & 0,77 & 0,62 & 0,87 & - \\
\hline
\end{tabular}

Palaeophyllophora. Toutefois, les caractères diagnostiques des Palaeophyllophora observables sur les spécimens du Chambi dénotent une probable affiliation avec le genre européen. Certains caractères comme la morphologie du cingulum lingual, la projection du talon, le talonide plus développé et la proportion du protocône se rapprochent plus des formes primitives telles qu' Icaronycteris et Archaeonycteris. Ces similitudes sont peut être la conséquence de l'ancienneté de ?P. tunisiensis Ravel, n. sp. qui aurait soit conservé certains traits plésiomorphiques des chiroptères, soit développé des convergences précoces avec les formes primitives.

\section{Genre Hipposideros Gray, 1831}

ESPĖCE TYPE. — Vespertilio speoris Schneider, 1800 par monotypie.

\section{Sous-genre Pseudorhinolophus Schlosser, 1887}

EsPèCe TYPe. - Pseudorhinolophus morloti Pictet in Pictet et al., 1855 par désignation subséquente (Schlosser 1887)

Autres espèces du sous-GenRe. - $H$. (Ps.) dubius Weithofer, $1887 ;$ H. (Ps.) weithoferi Revilliod, $1917 ;$ H. (Ps.) schlosseri Revilliod, 1917; H. (Ps.) egerkingensis Revilliod, 1922; H. (Ps.) bouziguensis Sigé, 1968; H. (Ps.) zbrjdi Sigé, 1990; H. (Ps.) russelli Maitre, 2014 ; H. (Ps.) major Maitre, 2014; H. (Ps.) tenuis Maitre, 2014.
Hipposideros (Pseudorbinolophus) africanum Ravel, n. sp. (Figs 8, 9, 10; Tableau 3)

HolOtyPe. - Spécimen CBI-1-247, fragment de maxillaire droit avec M2 et M3; Figs 8C; 9C.

Matériel eXAminé. - CBI-2-045 (Figs 8A; 9A), CBI-2-047 (Fig. 10B) et CBI-2-048 (C1s droites; Fig. 10A); CBI-2-046 (Figs 8B; 9B) et CBI-2-049 (C1s gauches); CBI-1-248 (M1 droite; Figs 8D; 9D); CBI-2-039 (Fig. 10C), CBI-2-043 (Figs 8E; 10D) et CBI-2-044 (M2s droites; Fig. 10E); CBI-2-041 (Fig. 10G) et CBI-2-042 (M3s droites; Fig. 10F); CBI-2-040 (M3 gauche); CBI2-053 (fragment de dentaire droite avec $\mathrm{m} 2$ et $\mathrm{m} 3$; Figs $8 \mathrm{~F}$; 9G); CBI-2-057 (Figs 8H; 9E) et CBI-2-058 (c1s droites; Fig. 10H); CBI-2-056 (c1 gauche; Figs 8I ; 9F); CBI-2-054 (Figs 8G; 9H) et CBI-2-055 (p2s droites; Fig. 10I); CBI-2-220 et CBI-3-004 (p2 gauche; Fig. 10I) ; CBI-2-050 (Figs 8J ; 10K) et CBI-2-051 (m1/2s droites; Fig. 9K) ; CBI-1-249 (Fig. 10J), CBI-2-250 (Figs 8K; 9J), et CBI-2-052 ( $\mathrm{m} 1 / 2 \mathrm{~s}$ gauches; Fig. $9 \mathrm{~L})$.

ÉTYMOLOGIE. - Le nom de l'espèce fait référence au continent africain et souligne l'originalité géographique de l'espèce qui appartient à un genre qui était documenté exclusivement en Europe.

LOCALITÉ TYPE ET ÂGE. - Chambi (CBI) loci 1, 2 et 3, Éocène inférieur terminal-Éocène moyen basal, Djebel Chambi, situé dans la région de Kasserine, Tunisie.

Diagnose. - Diffère des autres espèces d'Hipposideros (Pseudorhinolophus) par le faible développement et l'inclinaison distale du mésostyle, l'inclinaison mésiolinguale du bord buccal de M1, le cingulum lingual très épais et peu étendu, le développement à prédominance transversale de M3, et l'important développement longitudinal du talonide. 


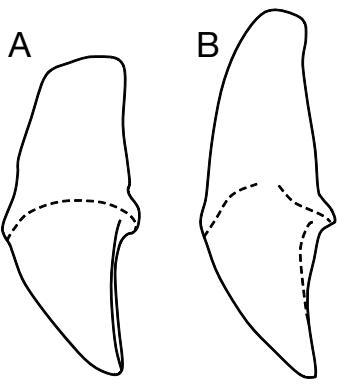

$\mathrm{F}$
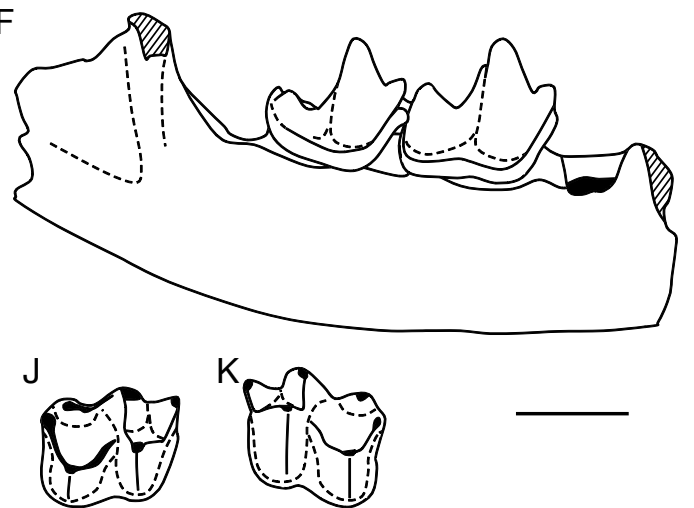

C
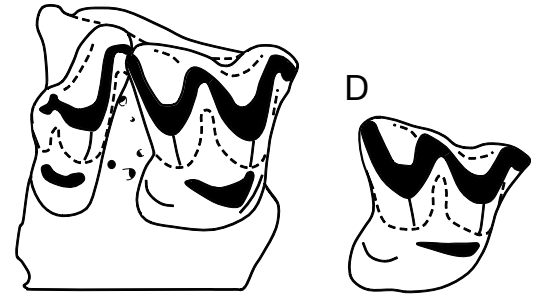

E

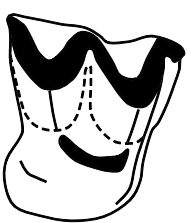

$\mathrm{H}$
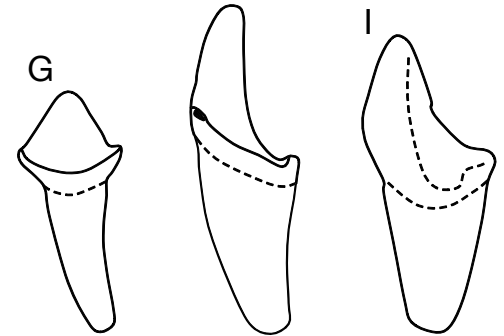

FIG. 8. - Hipposideros (Pseudorhinolophus) africanum Ravel, n. sp. provenant des loci 1 et 2 du Djebel Chambi dans la région de Kasserine, Tunisie: A, CBI-2045, C1 droite en vue linguale; B, CBI-2-046, C1 gauche en vue buccale; C, CBI-1-247, fragment de maxillaire avec M2 et M3 en vue occlusale; D, CBI-1-248, M1 droite en vue occlusale; E, CBI-2-043, M2 droite en vue occlusale; F, CBI-2-053, fragment de dentaire droite avec m2 et m3 en vue buccale; G, CBI-2-054, p2 droite en vue buccale; $\mathbf{H}, \mathrm{CBI}-2-057, \mathrm{c} 1$ droite en vue linguale; I, CBI-2-056, c1 gauche en vue buccale; J, CBI-2-050, m1/2 droite en vue occlusale; K, CBI$1-250, \mathrm{~m} 1 / 2$ gauche en vue occlusale. Échelle: $1 \mathrm{~mm}$.

\section{DESCRIPTION}

Les canines supérieures ont une morphologie simple (Figs 8A, B; 9A, B; 10A, B). L'unique tubercule est peu élevé, robuste, et montre un apex incliné distalement. La face buccale de la cuspide principale est bombée et s'oppose à une face linguale plane. Le cingulum est bien individualisé lingualement mais est absent sur la face buccale. Il forme à la base distale de la couronne un petit méplat (CBI-2-045 et CBI-2-046), ou forme un ressaut cuspidé relié par la crête distale du tubercule (CBI2-047 et CBI-2-049). La canine CBI-2-048 présente un cône moins incliné et une taille plus importante. Cette différence pourrait être liée soit à une variation intraspécifique, soit à la présence d'une autre espèce proche. Le manque de données ne permet pas de pencher pour l'une ou l'autre des hypothèses.

La première et la seconde molaire supérieure présentent de nettes différences morphologiques. La M1 est très compressée buccolingualement, ce qui lui confère un contour occlusal plutôt quadrangulaire (Figs 8D; 9D). Son bord buccal est incliné mésiolingualement et effectue un léger ectoflexus au dessus du paracône. Le parastyle est courbé vers l'avant, et apparait dès lors en position plus linguale que le métastyle, ce dernier étant plutôt rectiligne. Le « $V$ » antérieur de l'ectolophe, formé par la pré- et post- paracrêtes, est plus ouvert que le "V» postérieur (formé par les pré- et post- métacrêtes). Le mésostyle est peu développé et s'incline distalement sans se projeter au-delà du bord buccal. Le paracône est moins volumineux et plus lingual que le métacône. Le protocône est très effilé (comprimé buccolingualement) par rapport aux deux tubercules buccaux. Il est légèrement plus distal que le paracône. La préprotocrête s'étend buccalement jusqu'à la base du paracône, délimitant ainsi la paroi mésiale de la couronne. Le précingulum, très court, s'initie buccalement depuis la base du parastyle et s'étend distalement jusqu'à l'aplomb du paracône. La postprotocrête est environ deux fois plus longue que la préprotocrête. La postprotocrête s'étend vers la partie distale de manière quasi horizontale en vue occlusale. Elle s'arrête à la base linguale du métacône, laissant ainsi la protofosse ouverte distalement. Un cingulum lingual apparaît dans la région distolinguale de la couronne et prend la forme d'un épais lobe constituant le talon de la molaire.

Comparées à la M1, les M2s sont plus développées transversalement, avec un contour occlusal plus rectangulaire (Figs 8C-E; 9C, D; 10C-E). Le bord buccal est presque horizontal selon le plan occlusal, avec un léger ectoflexus au dessus du paracône (plus prononcé sur le spécimen CBI-2039). L'ectolophe de M2 est plus symétrique et plus large que celui de M1. Le parastyle est plus déjeté buccalement, se plaçant ainsi sur le même axe mésiodistal que le métastyle. Le mésostyle est réduit et légèrement en retrait du bord buccal. Le protocône est très compressé buccolingualement et moins allongé mésiodistalement que sur M1. Une partie du cingulum lingual est visible à la base du flanc mésial du protocône. Le lobe distolingual de M2 est moins projeté vers l'arrière que sur $M 1$. Un postcingulum vestigial s'étend depuis la base du métastyle jusqu'au point d'inflexion du lobe distolingual. 

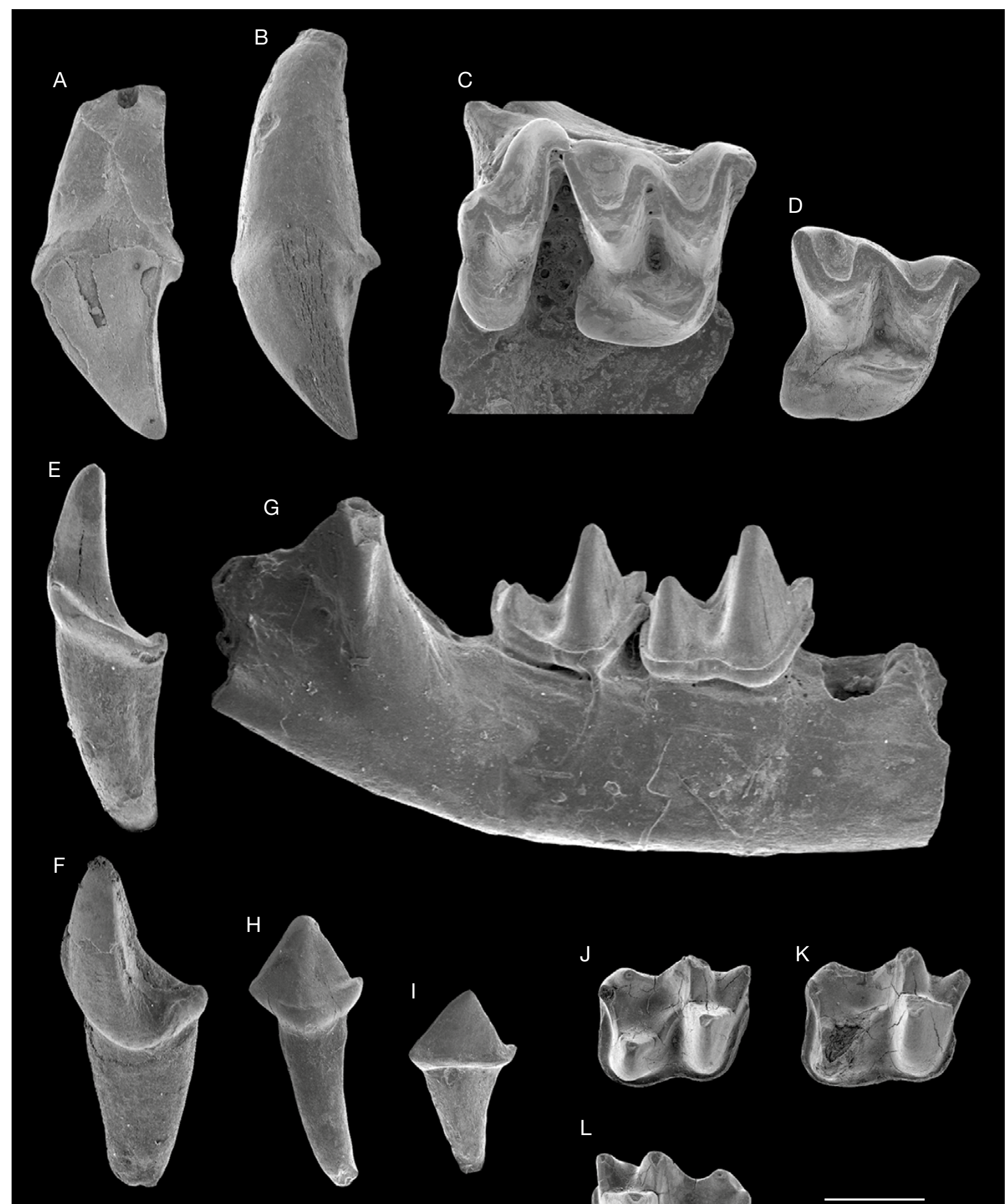

$\mathrm{J}$
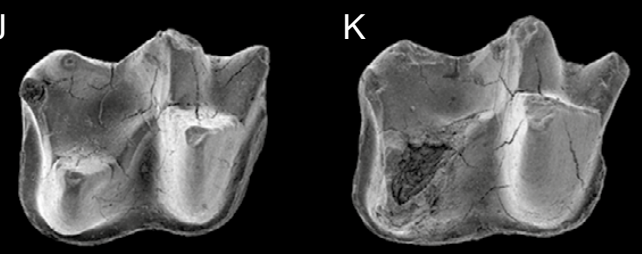

L

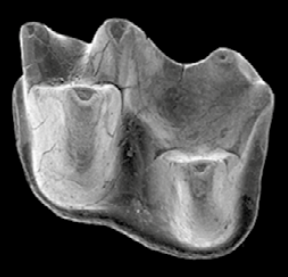

FIG. 9. - Hipposideros (Pseudorhinolophus) africanum Ravel, n. sp. provenant des loci 1 et 2 du Djebel Chambi dans la région de Kasserine, Tunisie: A, CBI-2045, C1 droite en vue linguale; B, CBI-2-046, C1 gauche en vue buccale; C, CBI-1-247, fragment de maxillaire droit avec M2 et M3 en vue occlusale; D, CBI1-248, M1 droite en vue occlusale; E, CBI-2-057, c1 droite en vue linguale; $\mathbf{F}, \mathrm{CBI}-2-056$, c1 gauche en vue buccale; G, CBI-2-053, fragment de dentaire droit avec $\mathrm{m} 2$ et $\mathrm{m} 3$ en vue buccale; $\mathbf{H}, \mathrm{CBI}-2-054$, p2 droite en vue buccale; I, CBI-2-055, p2 droite en vue linguale; J, CBI-1-250, m1/2 gauche en vue occlusale; K, CBI-2-051, m1/2 droite en vue occlusale; L, CBI-2-052, m1/2 gauche en vue occlusale. Échelle: $1 \mathrm{~mm}$. 


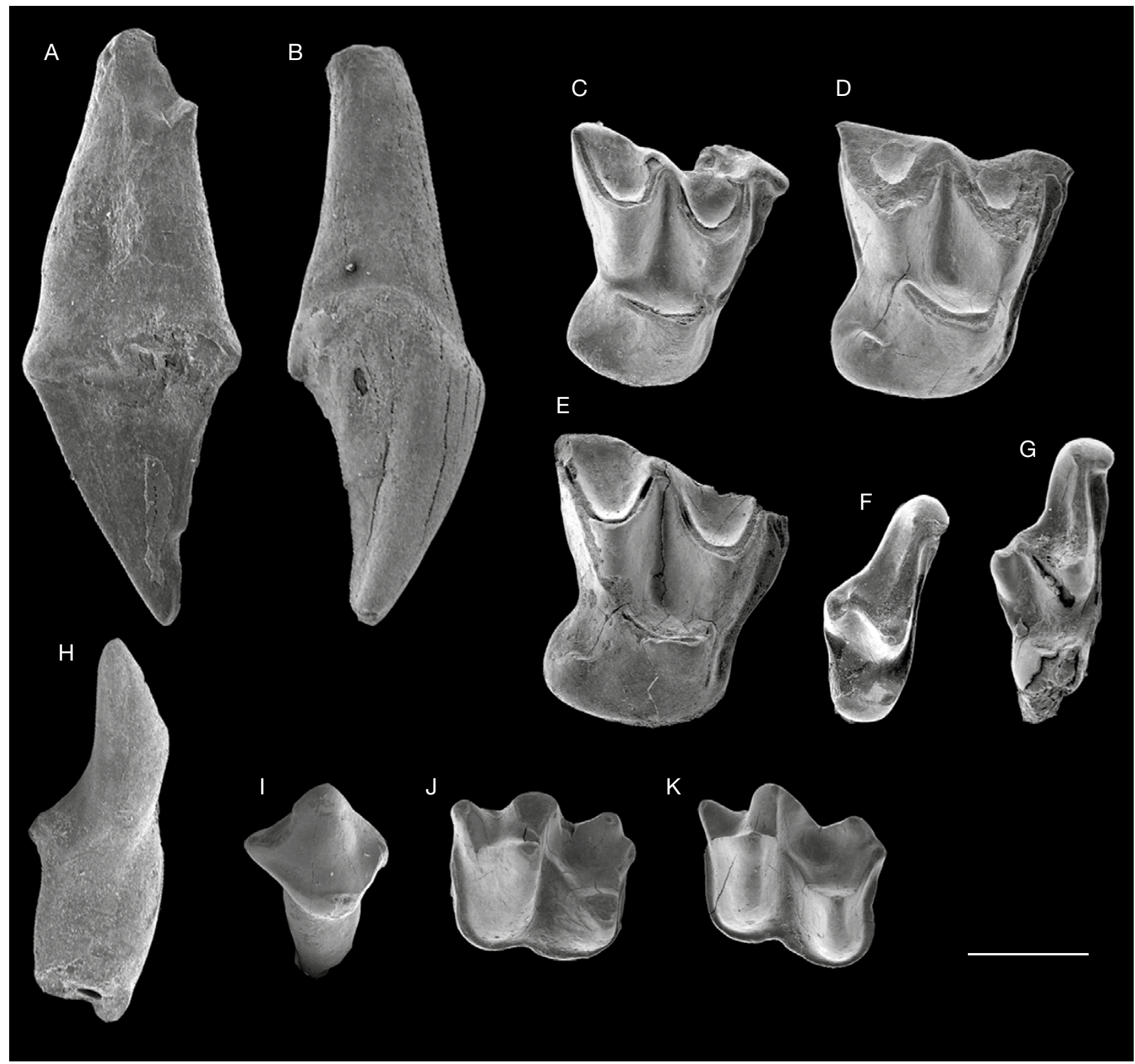

FiG. 10. - Hipposideros (Pseudorhinolophus) africanum Ravel, n. sp. provenant des loci 1, 2 et 3 du Djebel Chambi dans la région de Kasserine, Tunisie: A, CBI2-048, C1 droite en vue linguale; B, CBI-2-047, C1 droite en vue buccale; C, CBI-2-039, M2 droite en vue occlusale; D, CBI-2-043, M2 droite en vue occlusale; E, CBI-2-044, M2 droite en vue occlusale; F, CBI-2-042, M3 droite en vue occlusale; G, CBI-2-041, M3 droite en vue occlusale; $\mathbf{H}, \mathrm{CBI}-2-058$, c1 droite en vue buccale; I, CBI-3-004, p2 gauche en vue semi-buccale; J, CBI-1-249, m1/2 gauche en vue occlusale; $\mathbf{K}, \mathrm{CBI}-2-050$, m1/2 droite en vue occlusale. Échelle: 1 mm.

Les M3s ont une largeur équivalente aux M2s (Figs 8C; 9C; 10F, G). Ces molaires sont toutefois très courtes. Le bord buccal est très incliné distolingualement et infléchit au niveau du métacône. Le métacône est très peu développé et constitue un petit renflement distal. La prémétacrête est très courte tandis que la postmétacrête est absente. La partie linguale est dominée par le protocône dont le volume est inférieur à celui du paracône. Les protocrêtes sont très courtes et isolées. Le bord lingual arrondi est dépourvu de cingulum.

Deux molaires supérieures présentent une variation très forte. CBI-2-043 (Figs 8E; 10D) et CBI-2-044 (Fig. 10E) possèdent un très large précingulum s'étendant sur toute la largeur mésiale de la couronne depuis le parastyle. Cette structure est peu développée chez les autres spécimens. Cependant cette variation pourrait être due à une variation intraspécifique, mais ne peut, à elle seule, justifier la présence d'une autre espèce.

Les canines inférieures se composent d'un unique tubercule très légèrement recourbé (Figs $8 \mathrm{H}, \mathrm{I} ; 9 \mathrm{E}, \mathrm{F} ; 10 \mathrm{H})$. Le cingulum est bien marqué sur la face linguale tout en étant incliné distalement. Il forme un léger relief à la base mésiale de la cuspide et cerne un long bassin qui s'étend à la base distale du tubercule. Le cingulum est absent au niveau du bord buccal.

La deuxième prémolaire possède une couronne à contour ovale (Figs 8G; 9H, I; 10I). Elle se caractérise par le dével- 
oppement d'une seule cuspide, lequel constitue la majeure partie de la dent. Ce dernier est peu élevé, à base très large, et possède deux crêtes sagittales séparant la face buccale de la face linguale. La face buccale est légèrement plus bombée que la face linguale. Le cingulum, épais et continu, s'incurve buccalement. Un petit relief apparaît à la bordure distolinguale de la couronne formé en partie par un ressaut du cingulum.

Le talonide des molaires inférieures est très légèrement plus long que le trigonide (Figs 8F, J, K; 9G, J, K, L; 10J, K). Le trigonide est plutôt comprimé mésiodistalement: la distance entre le paraconide et le métaconide est moins importante que celle entre le protoconide et le paraconide ou le métaconide. Le paraconide est la cuspide du trigonide la plus réduite, il est également proclive. Le protoconide est droit, son flanc buccal est pincé mésiodistalement. Le talonide est plus large que le trigonide mais moins élevé en hauteur. L'hypoconide est le tubercule dominant du talonide. Il se situe plus buccalement que le protoconide. L'entoconide, de hauteur modérée par rapport aux autres tubercules du talonide, est légèrement plus distal que l'hypoconide. L'entocristide forme une encoche linguale entre l'entoconide et le métaconide. L'hypoconulide est proche distobuccalement de l'entoconide. Un petit hypoconulide, plus réduit que l'entoconide, est directement connecté à l'hypoconide par la postcristide dessinant ainsi une structure nettement nyctalodonte. La crête oblique commence depuis la base distale du trigonide entre le protoconide et le métaconide et rejoint la pointe de l'hypoconide. Le cingulide, modérément marqué, est continu sur tous les bords mésial, buccal et distal de la dent.

La m3 est plus petite que les $\mathrm{m} 1 \mathrm{~s}$ et $\mathrm{m} 2 \mathrm{~s}$ (Figs 8F ; 9G). Le trigonide de $\mathrm{m} 3$ n'est pas très modifié et se différencie par une taille plus petite et un resserrement mésiodistal du métaconide et du paraconide. La réduction de $\mathrm{m} 3$ est surtout réalisée au niveau du talonide. Cependant les trois cuspides du talonide sont bien visibles et de tailles similaires. L'hypoconulide est très projeté distalement à l'inverse de l'entoconide, lequel forme un petit bourrelet près de la base distale du métaconide. Le cingulide est également continu mais rétréci fortement au niveau du bord mésial et se termine buccalement à l'hypoconide.

\section{COMPARAISON ET DISCUSSION}

Ces fossiles de Chambi possèdent un ensemble de caractères qui se rencontrent plus généralement chez les Hipposideridae, et plus précisément du genre Hipposideros. Il s'agit notamment de:

- l'aspect comprimé buccolingualement des molaires supérieures;

- la morphologie de l'ectolophe;

- l'exiguïté de la protofosse;

- la proportion du protocône;

- l'extension du talon (lobe);

- des canines supérieures courbées et dépourvues de cingulum buccal;

- la présence d'un entoconide faible;

- la réduction latérale de la M3 (plus courant chez les Hipposideridae que chez les Rhinolophidae);
- la réduction du talonide de la m3 (également plus courant chez les Hipposideridae que chez les Rhinolophidae);

- l'extension de la postprotocrête.

Le genre Hipposideros est abondamment documenté dans les poches karstiques du Quercy depuis l'Éocène moyen terminal (Saint Lizier, MP16; une espèce reste non identifiée au niveau MP13 à Chamblon), jusqu'au début de l'Oligocène supérieur (Garouillas, MP25) et au Néogène (Sigé 1978, 1990; Sigé \& Legendre 1983; Maitre 2014). Ces fossiles ont été regroupés dans un sous-ensemble Hipposideros (Pseudorhinolophus), parfois utilisé comme genre à part entière (Hand 1998a, b) et dont la nomination rappelle les affinités morphologiques avec le genre Rhinolophus. Hipposideros (Pseudorhinolophus) africanum Ravel, n. sp. de Chambi partage avec les formes européennes :

- l'absence de cingulum buccal sur les canines;

- des faces lisses au tubercule des canines supérieures;

- le développement des crêtes internes de l'ectolophe;

- un paracône et un métacône de taille équivalente;

- la projection distale du talon de M1 plus importante par rapport à celle sur $\mathrm{M} 2$;

- la réduction de M3, avec la présence d'une arête distale au mésostyle;

- la présence d'un relief distolingual sur p2 (rudiment de paraconide);

- la réduction du talonide de $\mathrm{m} 3$.

L'ensemble de ces traits contribue à caractériser la morphologie dentaire des Hipposideros (Pseudorhinolophus), confirmant ainsi l'appartenance du taxon tunisien à ce sous-groupe. Hipposideros (Ps.) africanum Ravel, n. sp. possède également certaines divergences morphologiques avec les formes européennes, soulignant son identité spécifique:

- mésostyle moins projeté buccalement et incliné distalement ;

- forte inclinaison mésiolinguale du bord buccal de M1;

- M3 relativement plus développée transversalement;

- épaisseur du cingulum lingual sur M1-2 plus importante avec une extension mésiale plus réduite;

- extension du précingulum le long de la bordure mésiale de la couronne (variable parmi le matériel référé à $H$. [Ps.] africanum Ravel, n. sp.);

- tubercules des molaires inférieures mieux individualisés.

Hipposideros (Pseudorhinolophus) africanum Ravel, n. sp. témoigne de la présence de l'ensemble Pseudorhinolophus dès la fin de l'Éocène inférieur en Afrique. Les espèces européennes du groupe ne sont documentées qu'à partir de la fin de l'Éocène moyen. Cette espèce représente donc l'occurrence la plus précoce. Il est possible que le taxon tunisien, de par son ancienneté et son originalité vis-à-vis des autres espèces du même genre et sous-genre, reflète une évolution de la morphologie dont les tendances seraient au sein de cet ensemble: développement du mésostyle, redressement du bord buccal de $\mathrm{M} 1$, affinement et extension du cingulum lingual, réduction de M3, renforcement des cuspides des molaires inférieures, raccourcissement du talonide. Cependant le matériel reste insuffisant pour préciser les modalités macroévolutives des formes Hipposideros. 


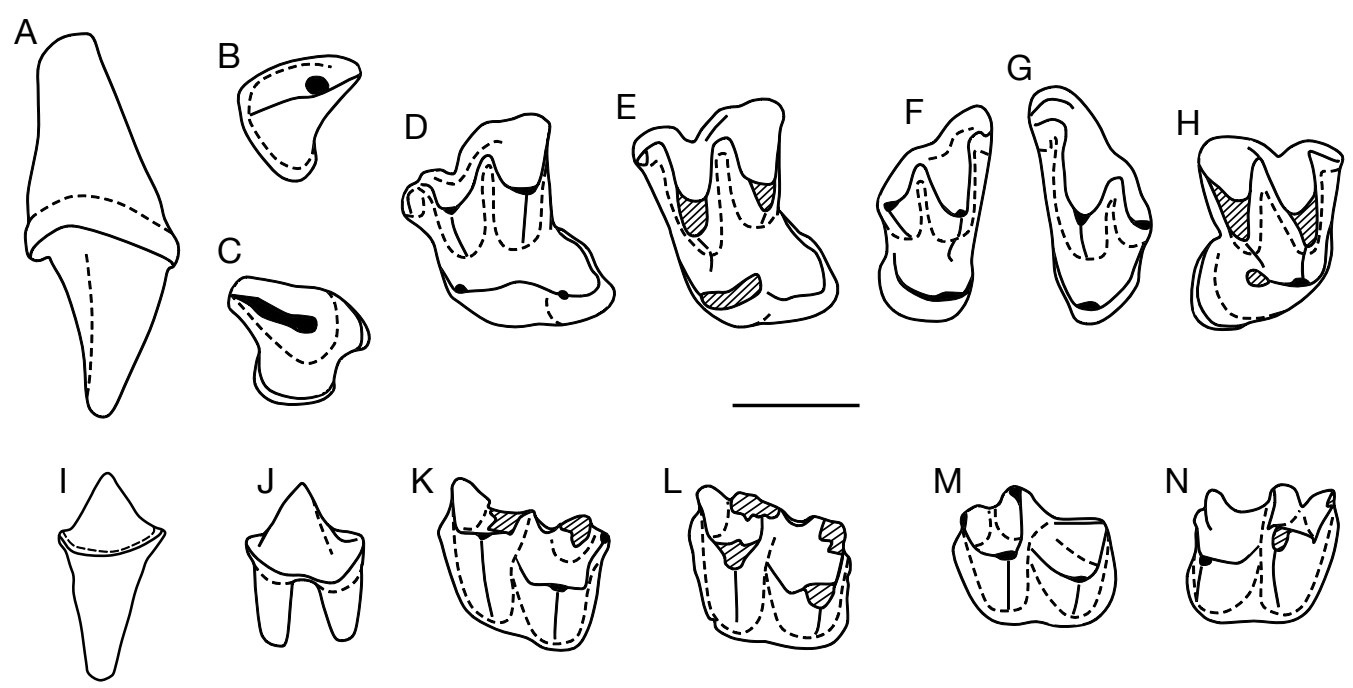

FIG. 11. - Vespertiliavus kasserinensis Ravel, n. sp. provenant des loci 1 et 2 du Djebel Chambi dans la région de Kasserine, Tunisie: A, CBI-2-072, C1 droite en vue buccale; B, CBI-2-068, P4 gauche en vue occlusale; C, CBI-2-070, P4 droite en vue occlusale; D, CBI-2-061, M1 gauche en vue occlusale; E, CBI-2-062, M2 gauche en vue occlusale; F, CBI-2-065, M3 droite en vue occlusale; G, CBI-2-066, M3 gauche en vue occlusale; $\mathbf{H}, \mathrm{CBI}-2-060$, M1 droite en vue occlusale; I, CBI-2-085, p2 gauche en vue buccale; J, CBI-1-251, p3 gauche en vue buccale; K, CBI-2-074, m1/2 gauche en vue occlusale; L, CBI-2-075, m1/2 gauche en vue occlusale; $\mathbf{M}, \mathrm{CBI}-2-078, \mathrm{~m} 3$ gauche en vue occlusale; $\mathbf{N}, \mathrm{CBI}-2-081, \mathrm{~m} 1 / 2$ droite en vue occlusale. Échelle: $1 \mathrm{~mm}$.

\section{Super-famille EMBALlOnUROIDEA Dobson, 1875 Famille EMBALlONURIDAE \\ Gervais in de Castelnau, 1855}

\section{Genre Vespertiliavus Schlosser, 1887}

ESPÈCE TYPE. — Vespertiliavus bourguignati Filhol, 1877 par désignation subséquente (Schlosser 1887).

AUTRES ESPÈCES DU GENRE. - V. wingei Revilliod, $1920 ; V$. schlosseri Revilliod, 1920 ; V. gracilis Revilliod, 1920 ; V. lapradensis Sigé, $1990 ;$ V. gerscheli Sigé, 1990; V. disjunctus Maitre, 2014; V. (Sigea) lizierensis Maitre, 2014; V. (Sigea) recens Maitre, 2014.

\section{Vespertiliavus kasserinensis Ravel, n. sp.}

(Figs 11, 12, 13; Tableau 4)

HolotyPE. - Spécimen CBI-2-061, M1 gauche, Figs 11D; 12D.

Matériel eXAminé. - CBI-2-072 (Figs 11A; 12A), CBI-2-096, CBI-2-097 et CBI-2-100 (C1s droites); CBI-2-073, CBI-2-098, CBI-2-099 et CBI-2-101 (C1s gauches); CBI-2-070 (P4 droite; Figs 11C; 12C); CBI-2-068 (Figs 11B; 12B), CBI-2-069 (Fig. 13A) et CBI-2-071 (P4s gauches; Fig. 13B) ; CBI-2-059 (Fig. 13G) et CBI-2-060 (M1s droites; Figs 11H; 13H) ; CBI-2-063 (Fig. 13C), CBI-2-064 (Fig. 13F), CBI-2-089, CBI-2-090 et CBI-3-005 (M1s gauches); CBI-2-062 (Figs 11E; 12E) et CBI-2-091 (M2s gauches); CBI-2-092 et CBI-2-093 (ectolophes de M2s droites); CBI-2094 (ectolophes de M2s gauches); CBI-2-065 (Figs 11F ; 12F), CBI-2-067 (Fig. 13D) et CBI-2-095 (M3s droites); CBI-2-066 (M3 gauche; Figs 11G; 13E) ; CBI-1-254 (fragment de dentaire gauche associé aux deux molaires inférieures isolées CBI-1-255 et -256); CBI-2-088 (c1?); CBI-2-086 et CBI-2-087 (p2s droites); CBI-2-085 (p2 gauche; Fig. 11I); CBI-2-082 et CBI-2-083 (p3s droites) ; CBI-1-251 (Figs 11J; 12G), CBI-2-084 et CBI-3-006 (p3s gauches); CBI-2-080 (Fig. 13K), CBI-2-081 (Figs 11N; 13L), CBI-2-108 et CBI-2-110 (m1/2s droites); CBI-2-074 (Figs $11 \mathrm{~K}$; 12H), CBI-2-075 (Figs 11L; 12I), CBI-2-076 (Fig. 13I), CBI-2-077 (Fig. 13J),CBI-2-104, CBI-2-105 et CBI-1-255 (m1/2s gauches);
CBI-2-106 et CBI-2-111 (m3s droites); CBI-2-078 (Figs 11M; 12J), CBI-2-079 (Fig. 13M), CBI-2-102, CBI-2-109, CBI-2-112, CBI-1-253 et CBI-1-256 (m3s gauches).

ÉTYMOLOGIE. - Le nom de l'espèce «kasserinensis» fait référence à Kasserine, ville située au pied du Djebel Chambi.

LOCALITÉ TYPE ET ÂGE. - Chambi (CBI) loci 1, 2 et 3, Éocène inférieur terminal-Éocène moyen basal, Djebel Chambi, situé dans la région de Kasserine, Tunisie.

Diagnose. - Vespertiliavus de petite taille, présentant un profond ectoflexus sur M1-2, une inclinaison prononcée du bord buccal de ces mêmes molaires, un cingulum buccal épais, un parastyle très fort partiellement désolidarisé de la préparacrête sur M1, un renflement linguodistal du bassin du talon parfois accompagné du développement d'un hypocône.

\section{DESCRIPTION}

Vespertiliavus kasserinensis Ravel, n. sp. est documenté par un matériel abondant et constitue l'une des espèces dominantes de la faune de Chambi. Certains spécimens sont de petite taille (CBI-1-251 et CBI-1-255) mais ne présentent aucune particularité morphologique en comparaison des spécimens types. Cette différence de taille peut s'expliquer par une large variation intraspécifique pouvant être la conséquence d'un dimorphisme sexuel courant chez les chiroptères actuels. Il est également possible que ces quelques spécimens appartiennent à une espèce emballonuride plus petite mais similaire à $V$. kasserinensis Ravel, n. sp. Cette espèce hypothétique ne peut cependant pas être clairement identifiée par le matériel actuellement référencé.

La canine supérieure (Figs 11A; 12A), de hauteur modérée, est munie d'une cuspide principale légèrement recourbée distalement. La face buccale du tubercule est convexe alors que la face linguale est quasi plane (à peine concave). La base de la cuspide principale porte un cingulum bien marqué qui 


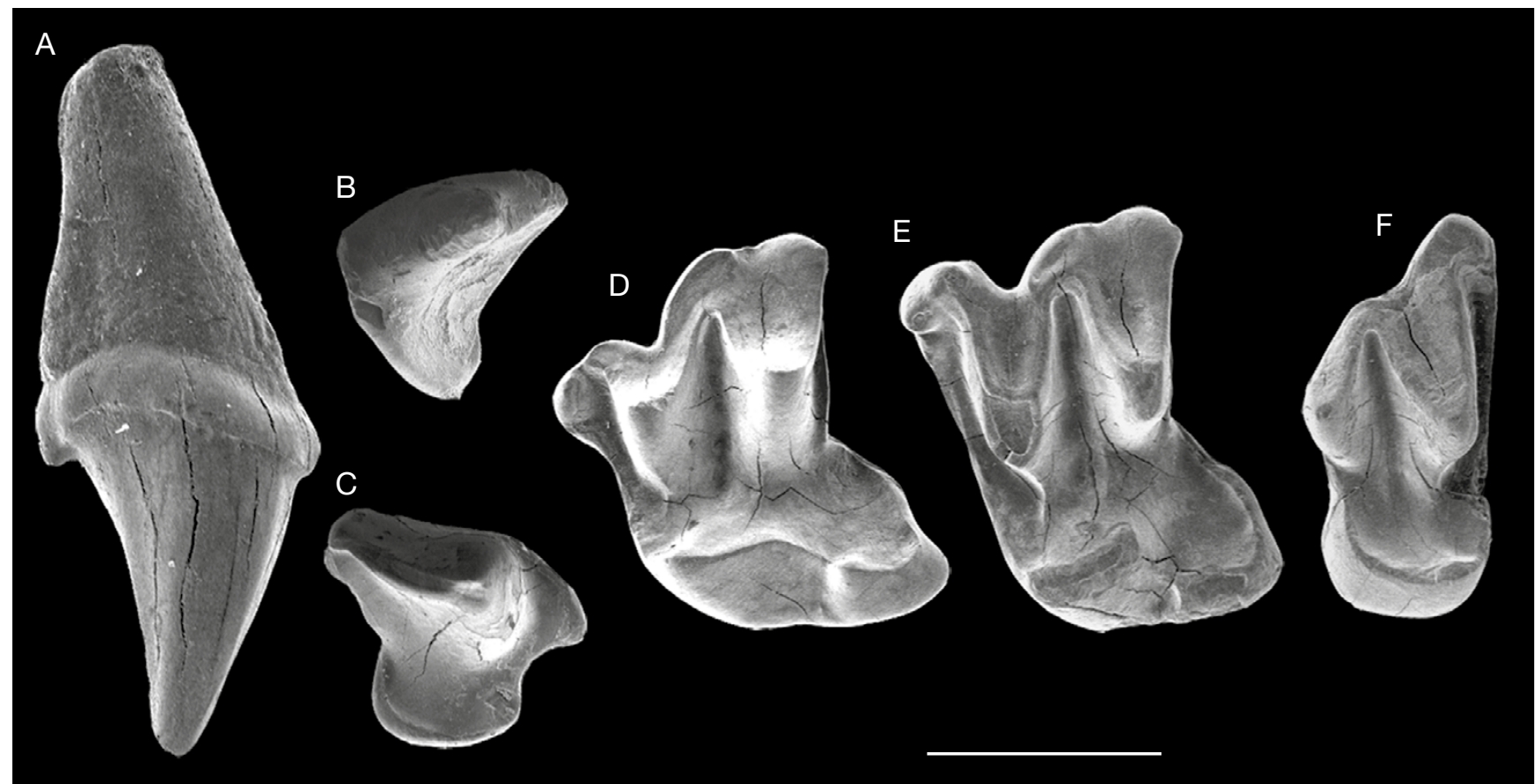

$\mathrm{H}$
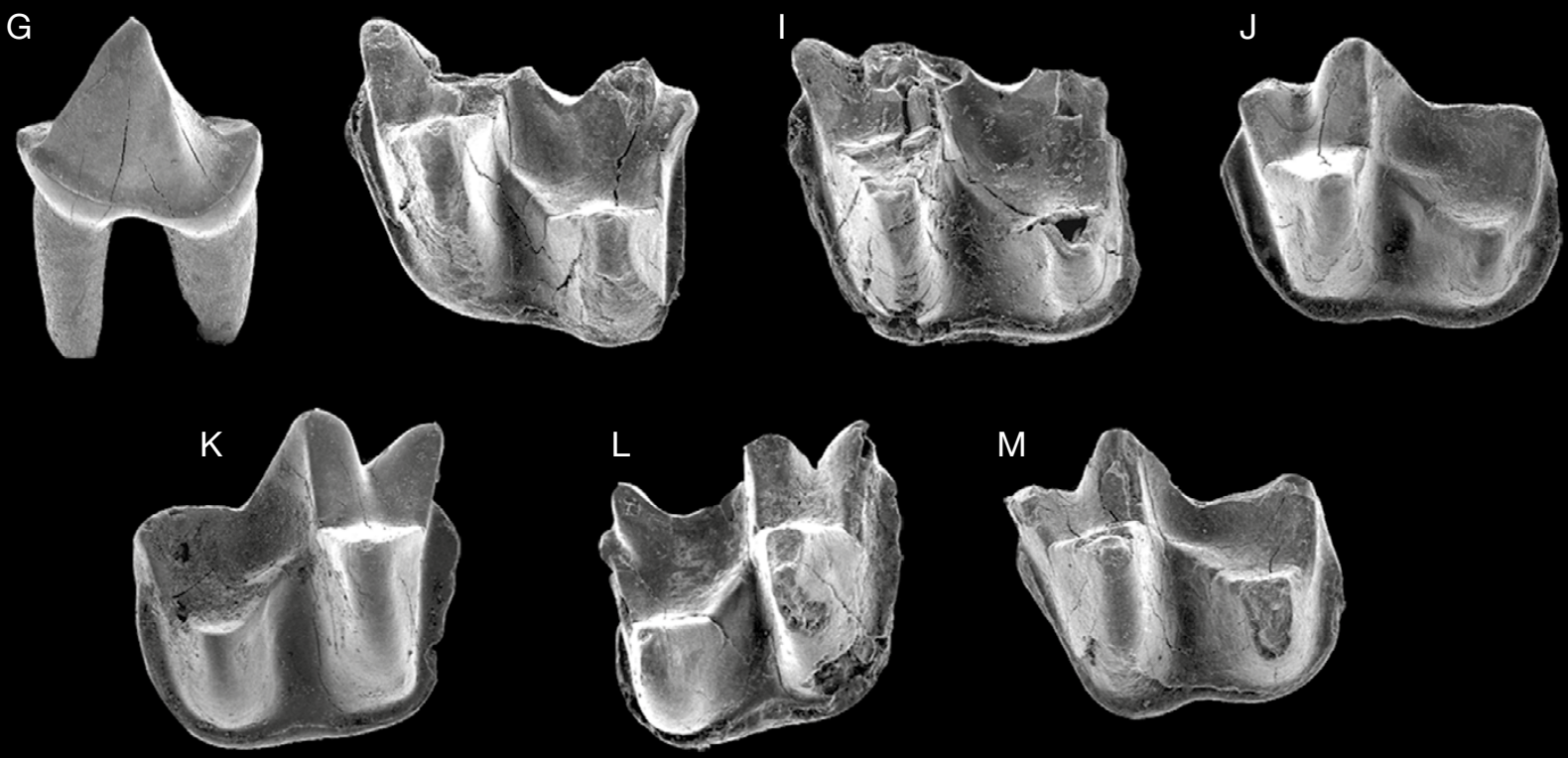

FIG. 12. - Vespertiliavus kasserinensis Ravel, n. sp. provenant des loci 1 et 2 du Djebel Chambi dans la région de Kasserine, Tunisie: A, CBI-2-072, C1 droite en vue buccale; B, CBI-2-068, P4 gauche en vue occlusale; C, CBI-2-070, P4 droite en vue occlusale; D, CBI-2-061, M1 gauche en vue occlusale; E, CBI-2-062, M2 gauche en vue occlusale; $\mathbf{F}, \mathrm{CBI}-2-065, \mathrm{M} 3$ droite en vue occlusale; $\mathbf{G}, \mathrm{CBI}-1-251$, p3 gauche en vue buccale; $\mathbf{H}, \mathrm{CBI}-2-074$, m1/2 gauche en vue occlusale; I, CBI-2-075, m1/2 gauche en vue occlusale; J, CBI-2-078, m3 gauche en vue occlusale; K, CBI-2-080, m1/2 droite en vue occlusale; L, CBI-2-081, m1/2 droite en vue occlusale; M, CBI-2-079, m3 gauche en vue occlusale. Échelle: $1 \mathrm{~mm}$.

occupe en continu toute la circonférence de la couronne. À l'extrémité la plus buccale de la couronne, ce cingulum est présent mais devient moins visible. Un petit conule initié par un ressaut du cingulum se positionne à l'extrémité distale de la couronne.

La P4 est triangulaire en vue occlusale, et présente un côté distolingual fortement incurvé vers l'intérieur de la dent (Figs 11B, C; 12B, C; 13A, B). Le paracône constitue l'unique relief cuspidé de la surface occlusale, et se positionne dans la partie centrobuccale de la couronne. Une crête débute depuis le sommet du paracône pour finir jusqu'à la limite distobuccale de la dent, tout en s'incurvant de façon très légère vers le bord buccal. La partie linguale de la prémolaire est dominée par un bassin peu étendu dans le sens de la largeur. Le bassin est cerné lingualement par un léger cingulum. Mésiobuccalement, les spécimens CBI-2-070 et CBI-2-071 possèdent un petit lobe 


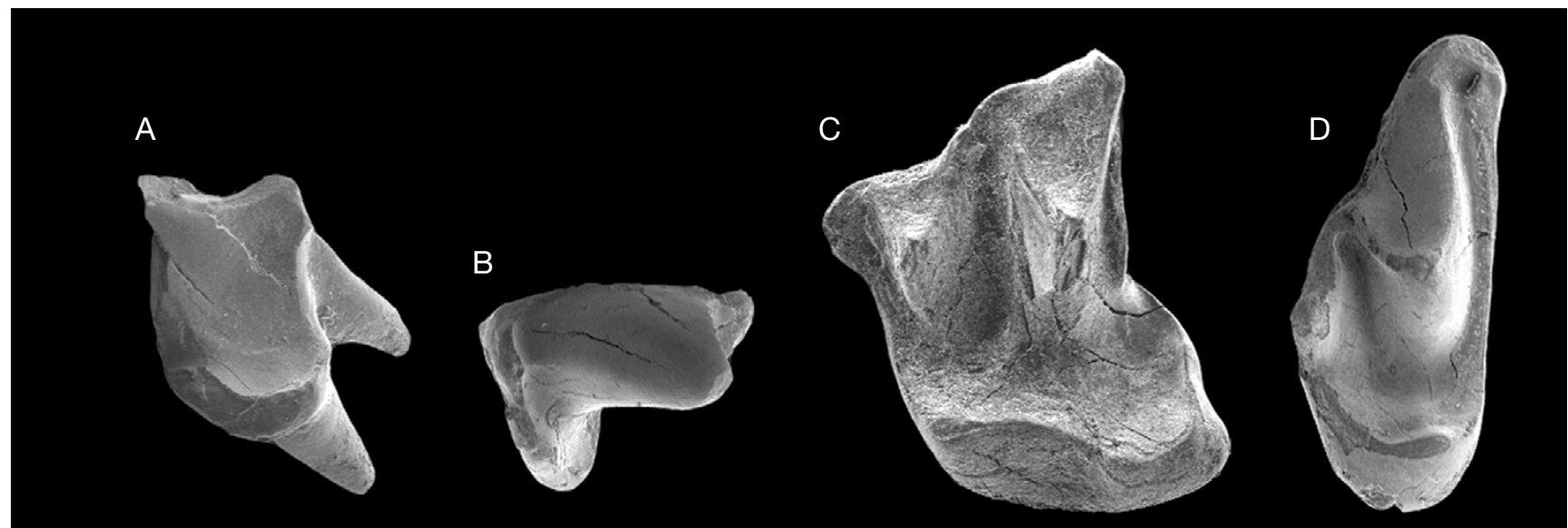

$\mathrm{E}$

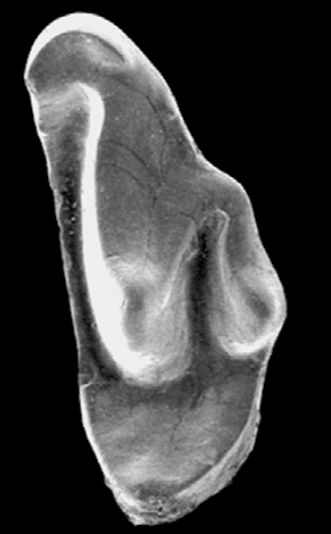

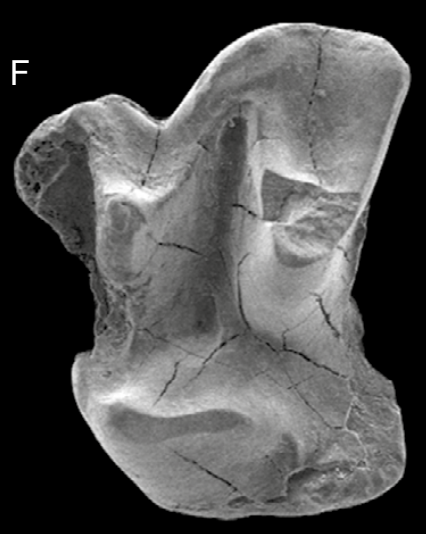

G

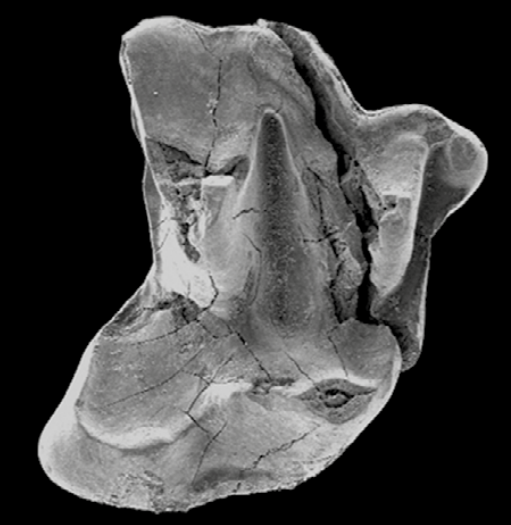

$\mathrm{H}$

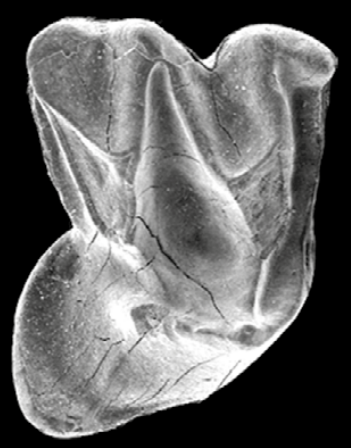

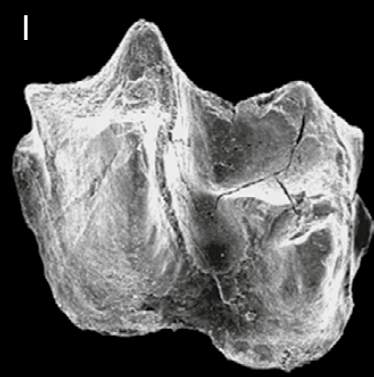

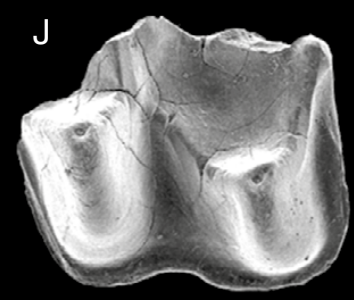

FIG. 13. - Vespertiliavus kasserinensis Ravel, n. sp. provenant du locus 2 du Djebel Chambi dans la région de Kasserine, Tunisie: A, CBI-2-069, P4 gauche en vue distolinguale; $\mathbf{B}, \mathrm{CBI}-2-071$, P4 gauche en vue mésiobuccale; $\mathbf{C}, \mathrm{CBI}-2-063, \mathrm{M} 1$ gauche en vue occlusale; $\mathbf{D}$, CBI-2-067, M3 droite en vue occlusale; E, CBI-2-066, M3 gauche en vue occlusale; F, CBI-2-064, M1 gauche en vue occlusale; G, CBI-2-059, M1 droite en vue occlusale; H, CBI-2-060, M1 droite en vue occlusale; I, CBI-2-076, m1/2 gauche en vue occlusale; J, CBI-2-077, m1/2 gauche en vue occlusale. Échelle: $1 \mathrm{~mm}$.

prenant la forme d'un méplat assez court. Ce méplat est plus individualisé sur CBI2-070 que sur CBI-2-071. Un matériel plus abondant permettrait de mieux cerner la nature de cette variation: intraspécifique ou interspécifique.

La M1 possède un contour occlusal plutôt compressé buccolingualement mais relativement allongé en raison du fort développement distal du talon (Figs 11D; 12D; 13C, F, G). Le bord buccal est fortement incliné mésiolingualement. Il effectue un fléchissement lingual abrupt (ectoflexus) surplombant le paracône. Le parastyle, qui fléchit mésialement pour former un lobe mésiobuccal très prononcé, est en position nettement plus linguale que le mésostyle et le métastyle. Le parastyle est dissocié de la préparacrête par une profonde mais étroite échancrure. La séparation entre le parastyle et la préparacrête est moins marquée sur les autres spécimens où ils sont même parfois connectés. Le mésostyle est peu développé et se situe en retrait par rapport au bord buccal. Le cingulum buccal est fort et forme une haute muraille surmontant la région mésostylaire. Le métastyle est crestiforme et constitue le prolongement buccal de la postmétacrête. L'orientation buccolinguale (quasi transversale) des post- et prémetacrêtes et des post- et préparacrêtes, ainsi que les styles associés qui les prolongent buccalement, a pour résultat un ectolophe fortement dissymétrique et comprimé distalement. Les pré- 
TABlEAU 4. - Mesures dentaires (en mm) de Vespertiliavus kasserinensis Ravel, n. sp. provenant des loci 1, 2 et 3 du Djebel Chambi dans la région de Kasserine, Tunisie. Abréviations: L, longueur; I, largeur; H, hauteur; Ltdr, longueur du trigonide; Itdr, largeur du trigonide; Ltdl, longueur du talonide; Itdl, largeur du talonide. Le signe * derrière une valeur indique un biais dans la mesure causé par l'altération de la couronne.

\begin{tabular}{|c|c|c|c|c|c|c|c|c|c|c|}
\hline Spécimen & Localité & $\begin{array}{l}\text { Type de } \\
\text { matériel }\end{array}$ & $\mathbf{L}$ & I & $\mathbf{H}$ & Ltrd & Itrd & Ltld & Itld & Remarques \\
\hline CBI-2-072 & Chambi 2 & C1 droite & 1,23 & 0,72 & 1,74 & - & - & - & - & - \\
\hline CBI-2-096 & Chambi 2 & C1 droite & 1,23 & 0,74 & $1,4^{*}$ & - & - & - & - & apex cassé \\
\hline CBI-2-097 & Chambi 2 & C1 droite & 1,17 & 0,69 & 1,33 & - & - & - & - & - \\
\hline CBI-2-100 & Chambi 2 & C1 droite & 0,98 & 0,61 & 1,74 & - & - & - & - & - \\
\hline CBI-2-073 & Chambi 2 & C1 gauche & 0,98 & 0,75 & $1,67^{*}$ & - & - & - & - & apex cassé \\
\hline CBI-2-098 & Chambi 2 & C1 gauche & 1,22 & 0,79 & $1,5^{\star}$ & - & - & - & - & apex cassé \\
\hline CBI-2-099 & Chambi 2 & C1 gauche & 1,1 & 0,7 & 1,61 & - & - & - & - & - \\
\hline CBI-2-101 & Chambi 2 & C1 gauche & 1,18 & 0,69 & 1,58 & - & - & - & - & - \\
\hline CBI-2-070 & Chambi 2 & P4 droite & 1,16 & 0,93 & - & - & - & - & - & - \\
\hline CBI-2-068 & Chambi 2 & P4 gauche & 1,04 & 0,87 & - & - & - & - & - & - \\
\hline CBI-2-069 & Chambi 2 & P4 gauche & 0,9 & 0,88 & - & - & - & - & - & - \\
\hline CBI-2-071 & Chambi 2 & P4 gauche & 1,14 & 0,93 & - & - & - & - & - & - \\
\hline CBI-2-059 & Chambi 2 & M1 droite & 1,91 & 1,88 & - & - & - & - & - & - \\
\hline CBI-2-060 & Chambi 2 & M1 droite & 1,4 & 1,63 & - & - & - & - & - & - \\
\hline CBI-2-061 & Chambi 2 & M1 gauche & 1,84 & 1,78 & - & - & - & - & - & - \\
\hline CBI-2-063 & Chambi 2 & M1 gauche & 1,7 & 1,89 & - & - & - & - & - & - \\
\hline CBI-2-064 & Chambi 2 & M1 gauche & $1,64^{*}$ & 1,87 & - & - & - & - & - & talon altéré \\
\hline CBI-2-089 & Chambi 2 & M1 gauche & 1,76 & 1,78 & - & - & - & - & - & - \\
\hline CBI-2-090 & Chambi 2 & M1 gauche & 1,81 & 1,88 & - & - & - & - & - & - \\
\hline CBI-3-005 & Chambi 3 & M1 gauche & $1,72^{*}$ & 2,17 & - & - & - & - & - & extrêmité distale du talon cassée \\
\hline CBI-2-094 & Chambi 2 & M2 droite & $1,37^{\star}$ & $1,28^{*}$ & - & - & - & - & - & ectolophe uniquement \\
\hline CBI-2-144 & Chambi 2 & M2 droite & $1,44^{*}$ & 1,78 & - & - & - & - & - & - \\
\hline CBI-2-062 & Chambi 2 & M2 gauche & 1,76 & 1,96 & - & - & - & - & - & - \\
\hline CBI-2-091 & Chambi 2 & M2 gauche & $1,18^{\star}$ & $2,08^{*}$ & - & - & - & - & - & $\begin{array}{l}\text { couronne altérée avec partie linguale } \\
\text { cassée }\end{array}$ \\
\hline CBI-2-092 & Chambi 2 & M2 gauche & $1,43^{*}$ & $1,39^{*}$ & - & - & - & - & - & ectolophe uniquement \\
\hline CBI-2-093 & Chambi 2 & M2 gauche & $1,48^{*}$ & $1,40^{*}$ & - & - & - & - & - & ectolophe uniquement \\
\hline CBI-2-065 & Chambi 2 & M3 droite & 0,96 & 1,83 & - & - & - & - & - & - \\
\hline CBI-2-067 & Chambi 2 & M3 droite & 0,96 & 2,01 & - & - & - & - & - & - \\
\hline CBI-2-095 & Chambi 2 & M3 droite & $0,83^{*}$ & 1,85 & - & - & - & - & - & $\begin{array}{l}\text { partie distale cassée, métacône } \\
\text { manquant }\end{array}$ \\
\hline CBI-2-066 & Chambi 2 & M3 gauche & 0,99 & 1,99 & - & - & - & - & - & - \\
\hline CBI-2-088 & Chambi 2 & $\mathrm{c} 1 ?$ & 0,93 & 0,76 & - & - & - & - & - & $\begin{array}{l}\text { seule la partie basale de la couronne } \\
\text { est conservée }\end{array}$ \\
\hline CBI-2-086 & Chambi 2 & p2 droite & 0,82 & 0,42 & - & - & - & - & - & - \\
\hline CBI-2-087 & Chambi 2 & p2 droite & 0,81 & $0,52^{*}$ & - & - & - & - & - & couronne altérée \\
\hline CBI-2-085 & Chambi 2 & p2 gauche & 0,87 & 0,51 & - & - & - & - & - & - \\
\hline CBI-2-082 & Chambi 2 & p3 droite & 1,1 & 0,48 & - & - & - & - & - & - \\
\hline CBI-2-083 & Chambi 2 & p3 droite & 1,26 & 0,54 & - & - & - & - & - & - \\
\hline CBI-1-251 & Chambi 1 & p3 gauche & 0,91 & 0,47 & - & - & - & - & - & - \\
\hline CBI-2-084 & Chambi 2 & p3 gauche & 1,26 & 0,59 & - & - & - & - & - & - \\
\hline CBI-3-006 & Chambi 3 & p3 gauche & 1,07 & 0,55 & - & - & - & - & - & - \\
\hline $\mathrm{CBI}-1-255$ & Chambi 1 & $\mathrm{~m} 2$ gauche & 1,01 & 0,72 & - & 0,58 & 0,66 & 0,43 & 0,72 & $\begin{array}{l}\text { m2 déraciné du fragment de dentaire } \\
\text { CBI-1-254 }\end{array}$ \\
\hline CBI-2-080 & Chambi 2 & $\mathrm{~m} 1 / 2$ droite & 1,36 & 0,93 & - & 0,72 & 0,93 & 0,64 & 0,77 & - \\
\hline $\mathrm{CBI}-2-081$ & Chambi 2 & $\mathrm{~m} 1 / 2$ droite & 1,28 & $0,86^{*}$ & - & 0,67 & 0,86 & 0,59 & $0,86^{\star}$ & $\begin{array}{l}\text { partie linguale cassée au niveau du } \\
\text { talonide }\end{array}$ \\
\hline CBI-2-110 & Chambi 2 & $\mathrm{~m} 1 / 2$ droite & - & - & - & - & - & 0,64 & 1,06 & $\begin{array}{l}\text { talonide complet, trigonide cassé } \\
\text { avec métaconide et une partie du } \\
\text { paraconide }\end{array}$ \\
\hline CBI-2-107 & Chambi 2 & $\mathrm{~m} 1 / 2$ droite & - & - & - & - & - & 0,85 & 0,85 & uniquement le talonide \\
\hline $\mathrm{CBI}-2-108$ & Chambi 2 & $\mathrm{~m} 1 / 2$ droite & - & - & - & 0,68 & 0,93 & & & $\begin{array}{l}\text { uniquement le trigonide et une partie } \\
\text { de l'hypoconide sont conservés }\end{array}$ \\
\hline CBI-2-074 & Chambi 2 & m1/2 gauche & 1,39 & 0,98 & - & 0,66 & 0,92 & 0,72 & 0,98 & \\
\hline $\mathrm{CBI}-2-075$ & Chambi 2 & $\mathrm{~m} 1 / 2$ gauche & $1,30^{\star}$ & 0,97 & - & 0,69 & 0,91 & $0,64^{*}$ & 0,97 & $\begin{array}{l}\text { extrêmité distale de la couronne } \\
\text { cassée }\end{array}$ \\
\hline CBI-2-076 & Chambi 2 & m1/2 gauche & $1,37^{*}$ & $0,90^{\star}$ & - & 0,73 & 0,9 & $0,64^{*}$ & $0,90^{\star}$ & $\begin{array}{l}\text { couronne très érodée avec structures } \\
\text { très émoussées }\end{array}$ \\
\hline CBI-2-077 & Chambi 2 & $\mathrm{~m} 1 / 2$ gauche & - & - & - & - & - & 0,72 & 0,88 & - \\
\hline CBI-2-104 & Chambi 2 & $\mathrm{~m} 1 / 2$ gauche & 1,37 & 0,81 & - & 0,72 & 0,81 & 0,65 & 0,63 & - \\
\hline CBI-2-105 & Chambi 2 & $\mathrm{~m} 1 / 2$ gauche & 1,24 & 0,87 & - & 0,68 & 0,84 & 0,56 & 0,87 & - \\
\hline CBI-2-103 & Chambi 2 & $\mathrm{~m} 1 / 2$ gauche & & & - & 0,7 & 0,85 & & & seul le trigonide est bien préservé \\
\hline CBI-1-252 & Chambi 1 & $\mathrm{~m} 1 / 2$ gauche & & & - & 0,68 & 0,76 & & & seul le trigonide est bien préservé \\
\hline CBI-2-106 & Chambi 2 & m3 droite & 1,37 & $0,85^{\star}$ & - & 0,78 & $0,85^{\star}$ & 0,59 & 0,64 & $\begin{array}{l}\text { couronne cassée aux extrêmités, } \\
\text { protocône en partie cassé }\end{array}$ \\
\hline CBI-2-111 & Chambi 2 & m3 droite & 1,23 & 0,88 & - & 0,67 & 0,88 & 0,55 & 0,68 & - \\
\hline CBI-2-078 & Chambi 2 & m3 gauche & 1,36 & 0,85 & - & 0,7 & 0,85 & 0,64 & 0,71 & - \\
\hline
\end{tabular}




\begin{tabular}{|c|c|c|c|c|c|c|c|c|c|c|}
\hline Spécimen & Localité & $\begin{array}{l}\text { Type de } \\
\text { matériel }\end{array}$ & $\mathbf{L}$ & I & H & Ltrd & Itrd & Ltld & Itld & Remarques \\
\hline CBI-2-079 & Chambi 2 & m3 gauche & 1,28 & 0,83 & - & 0,62 & 0,83 & 0,66 & 0,67 & - \\
\hline CBI-2-102 & Chambi 2 & m3 gauche & 1,23 & 0,78 & - & 0,65 & 0,78 & 0,59 & 0,69 & - \\
\hline CBI-2-109 & Chambi 2 & m3 gauche & 1,25 & $0,74^{*}$ & - & 0,66 & $0,74^{*}$ & 0,59 & $0,63^{*}$ & $\begin{array}{l}\text { partie linguale cassée au niveau du } \\
\text { talonide }\end{array}$ \\
\hline $\mathrm{CBI}-2-112$ & Chambi 2 & m3 gauche & 1,37 & 0,9 & - & 0,76 & 0,9 & 0,61 & 0,67 & - \\
\hline $\mathrm{CBI}-1-253$ & Chambi 1 & m3 gauche & 1,37 & 0,87 & - & 0,74 & 0,87 & 0,62 & 0,74 & - \\
\hline CBI-1-256 & Chambi 2 & m3 gauche & 0,94 & 0,58 & - & 0,49 & 0,58 & 0,45 & 0,5 & $\begin{array}{l}\text { m3 déracinée du fragment de } \\
\text { dentaire CBI-1-254 }\end{array}$ \\
\hline CBI-1-254 & Chambi 3 & $\begin{array}{l}\text { fragment } \\
\text { dentaire } \\
\text { gauche }\end{array}$ & 4,45 & 0,71 & - & - & - & - & - & $\begin{array}{l}\text { avec alvéloles de m2/m3 qui } \\
\text { correspondent avec CBI-1-255 } \\
\text { (m2) et CBI-1-256 (m3) }\end{array}$ \\
\hline
\end{tabular}

et post- paracrêtes forment un « $\mathrm{V}$ » antérieur plus ouvert que le «V» postérieur formé par les pré- et post- métacrêtes (ces dernières sont subparallèles l'une à l'autre). Le paracône est plus petit et plus lingual que le métacône. Un très court paralophe débute à la base linguale du paracône et progresse transversalement vers la protofosse. Le protocône, moins élevé mais plus volumineux que le paracône et le métacône, est déjeté sur la bordure mésiolinguale de la couronne. La préprotocrête est courte par rapport à la postprotocrête. La crête antérieure du protocône remonte buccalement pour faire la jonction avec le précingulum. Ce dernier est très épais par rapport au postcingulum très fin. La postprotocrête est beaucoup plus longue que la préprotocrête. Elle prend une forme sinusoïdale pour rejoindre directement l'hypocône quand il est présent (CBI-2-059 et CBI-2-061), lequel est peu développé. La protofosse est ainsi ouverte distalement sur un large et long bassin du talon. La connexion entre la protofosse et le bassin du talon confère à la molaire une surface occlusale très étendue. L'hypocône forme un tubercule très distal mais légèrement plus lingual par rapport au protocône. Le cingulum lingual, très fort, débute depuis l'hypocône et ceinture le bassin du talon sur toute sa partie distale. Il fini sa course en remontant buccalement jusqu'au postcingulum. Il s'épaissit fortement sur la bordure distolinguale de la couronne, formant un lobe très prononcé.

Comme la plupart des espèces appartenant à la famille des Emballonuridae, le taxon de Chambi présente une grande différence structurale entre les M1s et M2s. Les M2s ont un développement transversal alors que les M1s ont un développement plutôt longitudinal (Figs 11E; 12E). L'ectolophe des M2s est plus développé dans le sens buccolingual et plus resserré mésiodistalement. L'extension et la morphologie du talon de la M2 sont également très différents (i.e., plus court et moins bombé distolingualement). Le bord buccal est beaucoup moins incliné sur M2 que sur M1. Le parastyle occupe de ce fait une position plus buccale sur M2. Sur M2, le mésostyle est également plus buccal et se situe sur le même axe mésiodistal que le parastyle. Les crêtes de l'ectolophe sont de longueur similaire. Ces crêtes sont plus comprimées mésiodistalement et apparaissent ainsi quasi parallèles les unes aux autres. De ce fait, la forme en "W" de l'ectolophe apparait plus fermée sur M2 que sur M1. La différence de taille entre le métacône et le paracône est plus ténue sur la M2. La postprotocrête, orientée buccodistalement, est relativement plus courte. Cette crête n'est pas connectée à l'hypocône, et est interrompue au niveau de la protofosse. Comme sur M1, la protofosse est ouverte distalement et communique ainsi avec le large bassin du talon. La zone où se situe habituellement l'hypocône est érodée sur le seul spécimen complet de M2 (CBI-2-062; Figs 11E; $12 \mathrm{E})$. Cette cassure ne permet pas de confirmer si oui ou non l'hypocône est présent sur ce spécimen. Le bassin du talon est moins projeté distalement. Il ne forme pas un lobe distolingual comme c'est le cas sur M1.

La M3 est peu réduite par rapport aux M1 et M2 (Figs 11F, $\mathrm{G} ; 12 \mathrm{~F} ; 13 \mathrm{D}, \mathrm{E})$. Le bord buccal de la couronne est fortement incliné distolingualement avec un léger ressaut au niveau du mésostyle. Le parastyle est bien développé, et s'infléchit mésialement en forme de crochet. Le métacône est présent et bien développé, tout en étant à peine plus petit que le paracône. En revanche, il n'y a pas de postmétacrête ni de métastyle. Le protocône est très réduit et compressé buccolingualement. Comme sur M1 et M2, la préprotocrête rejoint le précingulum. La postprotocrête remonte buccalement et se connecte à la base linguale du métacône. Le postcingulum est absent. La couronne de M3 ne possède pas de talon, le bord lingual est simple et arrondi.

Un des spécimens attribuable à une $\mathrm{M} 1$ présente une forte variation morphologique (CBI-2-060; Figs $11 \mathrm{H}$; 12H). Le bord buccal de la molaire est beaucoup moins incliné par rapport aux autres M1s. L'ectoflexus est moins profond. Le paralophe et le métalophe sont très développés. Le paralophe est tranchant et rejoint directement l'apex du protocône. Le métalophe est également haut et tranchant. Il s'étend mésiolingualement mais ne rejoint pas la postprotocrête. La protofosse est cernée par les protocrêtes, le paralophe et le métalophe, réduisant ainsi considérablement son extension mésiale et distale. Le spécimen ne possède pas d'hypocône et son cingulum lingual est moins marqué. Ces différences pourraient témoigner de la présence éventuelle d'une autre espèce proche de $V$. kasserinensis Ravel, n. sp., plutôt que de l'existence d'une variation du morphotype. Mais en l'absence d'un matériel plus abondant il n'est pas possible d'infirmer cette éventualité. 
TABLEAu 5. - Mesures dentaires (en mm) de ?Vespertiliavus aenigma Ravel, n. sp. provenant des loci 1, 2 et 3 du Djebel Chambi dans la région de Kasserine, Tunisie. Abréviations: L, longueur; I, largeur; H, hauteur; Ltdr, longueur du trigonide; Itdr, largeur du trigonide; Ltdl, longueur du talonide; Itdl, largeur du talonide. Le signe * derrière une valeur indique un biais dans la mesure causé par l'altération de la couronne.

\begin{tabular}{|c|c|c|c|c|c|c|c|c|c|c|}
\hline Spécimen & Localité & $\begin{array}{l}\text { Type de } \\
\text { matériel }\end{array}$ & $\mathbf{L}$ & $\mathbf{I}$ & $\mathbf{H}$ & Ltrd & Itrd & Ltld & Itld & Remarques \\
\hline CBI-2-175 & Chambi 2 & C1 droite & 1,39 & 0,91 & $2,20^{*}$ & - & - & - & - & apex cassé \\
\hline CBI-2-176 & Chambi 2 & C1 gauche & 1,27 & 0,86 & $2,22^{\star}$ & - & - & - & - & couronne émoussée \\
\hline CBI-2-177 & Chambi 2 & C1 gauche & 1,35 & 0,91 & $2,11^{*}$ & - & - & - & - & couronne émoussée \\
\hline CBI-2-174 & Chambi 2 & P4 gauche & 1,28 & 1,03 & - & - & - & - & - & - \\
\hline CBI-2-169 & Chambi 2 & M1 droite & 2,2 & 2,08 & - & - & - & - & - & région du parastyle érodée \\
\hline CBI-2-170 & Chambi 2 & M1 gauche & $1,79^{*}$ & 2,13 & - & - & - & - & - & uniquement ectolophe \\
\hline CBI-2-171 & Chambi 2 & M2 droite & $1,51^{*}$ & $1,48^{*}$ & - & - & - & - & - & - \\
\hline CBI-3-013 & Chambi 3 & M3? gauche & 1,42 & 2,04 & - & - & - & - & - & - \\
\hline CBI-2-172 & Chambi 2 & M3? gauche & 1,37 & 2 & - & - & - & - & - & - \\
\hline CBI-2-173 & Chambi 2 & M3? droite & - & - & - & - & - & - & - & $\begin{array}{l}\text { ectolophe cassé, seul le métaconide } \\
\text { est préservé }\end{array}$ \\
\hline CBI-2-205 & Chambi 2 & c1 droite & 1,02 & 0,63 & $1,48^{\star}$ & - & - & - & - & apex cassé \\
\hline CBI-2-183 & Chambi 2 & p4 droite & 1,18 & 0,66 & - & - & - & - & - & - \\
\hline CBI-2-182 & Chambi 2 & p4 gauche & 1,28 & 0,72 & - & - & - & - & - & - \\
\hline CBI-2-178 & Chambi 2 & $\mathrm{~m} 1 / 2$ droite & 1,56 & 0,92 & - & 0,76 & 0,83 & 0,8 & 0,92 & - \\
\hline CBI-2-179 & Chambi 2 & $\mathrm{~m} 1 / 2$ droite & $1,53^{*}$ & 1,08 & - & $0,73^{*}$ & $0,80^{*}$ & 0,81 & 1,08 & - \\
\hline CBI-2-181 & Chambi 2 & $\mathrm{~m} 1 / 2$ droite & 1,79 & 1,07 & - & 0,89 & 1,07 & 0,9 & 1,07 & - \\
\hline CBI-1-262 & Chambi 1 & $\mathrm{~m} 1 / 2$ droite & 1,7 & 1,04 & - & 0,82 & 0,92 & 0,78 & - & - \\
\hline CBI-2-180 & Chambi 2 & $\mathrm{~m} 1 / 2$ gauche & 1,47 & 0,96 & - & 0,67 & $0,74^{*}$ & 0,8 & 0,96 & - \\
\hline
\end{tabular}

La couronne de p2 est très simple (Fig. 11I). En vue occlusale, la dent est ovale et étroite. Le tubercule unique, biface, constitue la principale structure de la dent. Deux crêtes sagittales débutent depuis l'apex peu élevé du conide pour rejoindre les extrémités mésiale et distale de la couronne. Un petit ressaut d'émail situé à chacune des extrémités marque la jonction des crêtes avec le cingulum. Ce dernier, d'épaisseur modérée, ceinture la base de la couronne sur tout son pourtour.

Est identifiée comme p3, une dent allongée mésiodistalement mais très étroite (par ex. : CBI-1-251 ; Fig. 11J ; 12G). La morphologie de cette quatrième prémolaire inférieure diffère de celle de p2 par la présence de deux racines, l'absence de crête distale, l'incurvation du bord buccal, et la présence d'un petit bassin situé à la base distale du tubercule principal.

Aucune p4 n'a été identifiée dans le matériel de Chambi. Cette absence peut s'expliquer par un biais d'échantillonnage ou par la non reconnaissance de la morphologie de ces prémolaires parmi le matériel encore non identifié. Dans ce dernier cas, des rangées dentaires complètes bien préservées seraient indispensables pour identifier des dents isolées, et décrire la morphologie de ce locus dentaire.

Plusieurs molaires inférieures attribuables à cette nouvelle espèce ont été identifiées. Cependant, il est à noter que peu de caractères nous permettent de distinguer de manière précise les $\mathrm{m} 1 \mathrm{~s}$ des $\mathrm{m} 2 \mathrm{~s}$ à partir du matériel disponible. Ces molaires inférieures ont toutes un contour rectangulaire (plus long que large) en vue occlusale (Figs $11 \mathrm{~K}, \mathrm{~L}, \mathrm{~N} ; 12 \mathrm{H}, \mathrm{I}, \mathrm{K}, \mathrm{L}$; 13I, $\mathrm{J})$. Leur trigonide est plus court et étroit que le talonide, mais beaucoup plus élevé. Les métaconide et paraconide sont relativement rapprochés l'un de l'autre et sont de taille équivalente. Ces deux cuspides sont plus réduites que le protoconide malgré le fort pincement mésiodistal de ce dernier. L'hypoconide est situé plus buccalement que le protoconide, ce qui induit un talonide plus large que le trigonide. Une longue crête oblique émerge depuis la base de la muraille distale du trigonide et rejoint l'hypoconide en s'inclinant distobuccalement. La postcristide connecte l'hypoconide à l'hypoconulide, définissant ainsi une structure nettement nyctalodonte. L'hypoconulide, réduit, est situé distalement et un peu plus buccalement par rapport à l'entoconide. L'entoconide est manquant (cassure post-mortem) sur la plupart des spécimens, certainement en raison de sa fragilité (effilé et saillant). L'entocristide débute depuis la base distolinguale du métaconide et s'incurve fortement distalement pour rejoindre le sommet de l'entoconide. Le cingulide est continu sur les bordures mésiale, buccale et distale de la couronne.

La m3 est peu réduite par rapport aux autres molaires, et apparaît un peu plus courte (Figs 11M; 12J). La morphologie du trigonide ne change pas significativement. Le talonide reste plus large que le trigonide même si la différence en largeur et longueur est moins importante. L'entoconide est relativement haut et l'hypoconulide n'apparaît pas. Le petit tubercule manquant, la postcristide rejoint directement l'entoconide. Comme sur $\mathrm{m} 1 / 2$, le cingulide est continu sur les bordures mésiale, buccale et distale.

\section{COMPARAISON ET DISCUSSION}

Comme la plupart des familles chiroptériennes, la définition des Emballonuridae se base sur des critères diagnostiques crâniens et postcrâniens (Miller 1907). La morphologie dentaire des Emballonuridae est toutefois bien identifiable par:

- la présence d'un cingulum bien individualisé sur les canines supérieures;

- le relief distal des canines supérieures formant un petit conule; - la compression buccolinguale des P4s (contour occlusal triangulaire);

- le parastyle des M1s déplacé lingualement, entrainant une inclinaison modérée à forte du bord buccal ;

- le bassin du talon des molaires supérieures (M1/2) très étendu distalement et linguobuccalement; 

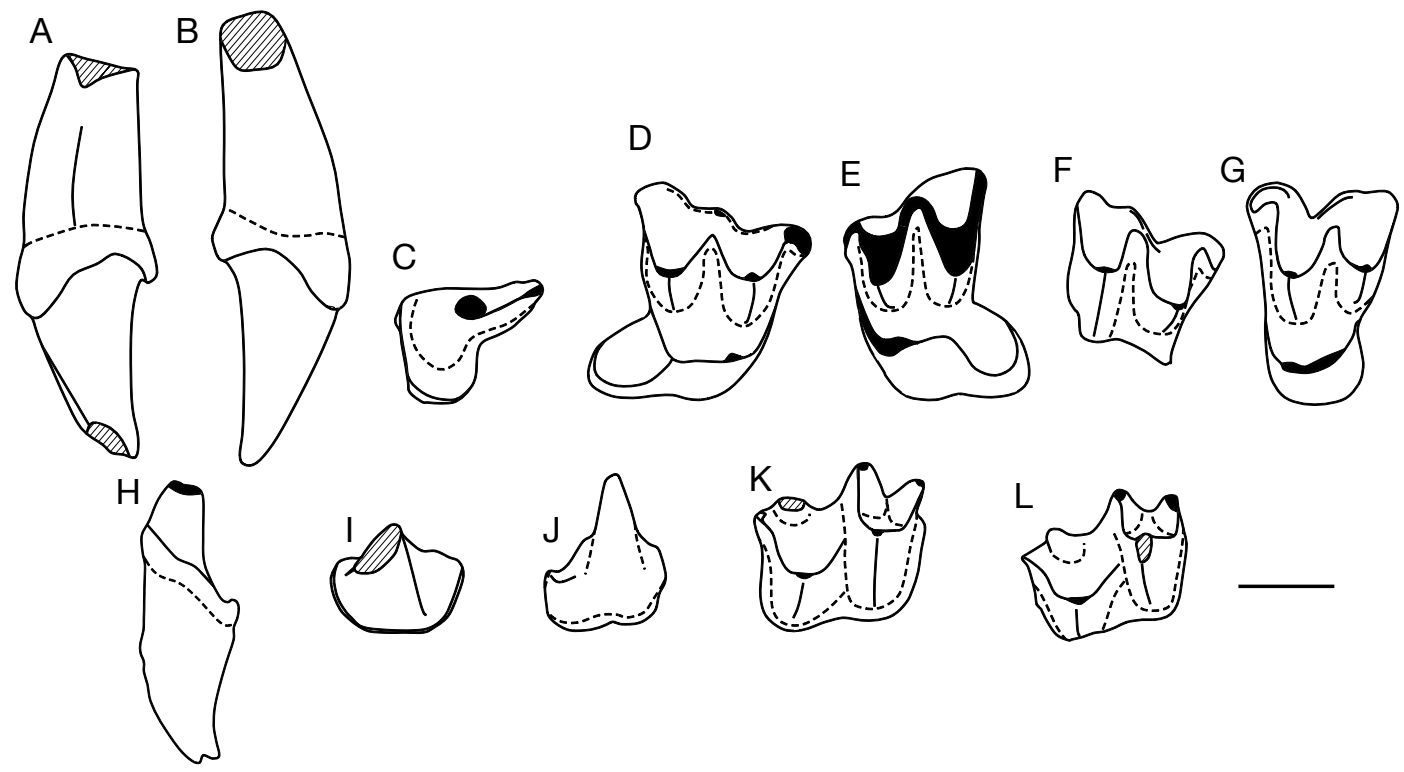

FIG. 14. - ?Vespertiliavus aenigma Ravel, n. sp. provenant des loci 2 et 3 du Djebel Chambi dans la région de Kasserine, Tunisie: A, CBI-2-175, C1 droite en vue linguale; B, CBI-2-176, C1 gauche en vue linguale; C, CBI-2-174, P4 gauche en vue occlusale; D, CBI-2-169, M1 droite en vue occlusale; E, CBI-2-170, M1 gauche en vue occlusale; F, CBI-2-171, ectolophe de M2 droite en vue occlusale; G, CBI-3-013, M3 gauche en vue occlusale; $\mathbf{H}$, CBI-2-205, c1 droite en vue linguale; I, CBI-2-182, p4 gauche en vue semi buccale; J, CBI-2-183, p4 droite en vue buccale; K, CBI-2-181, m1/2 droite en vue occlusale; L, CBI-2-178, $\mathrm{m} 1 / 2$ droite en vue occlusale. Échelle: $1 \mathrm{~mm}$.

- la présence d'un hypocône;

- la postprotocrête orientée distalement rejoignant parfois l'hypocône ou le cingulum lingual;

- la communication entre la protofosse et le bassin du talon, conférant aux M1 et M2 une large surface occlusale;

- la M3 bien développée et muni d'un métacône bien défini; - la compression mesiodistale du trigonide des molaires inférieures, formant un « $V$ » refermé en vue occlusale;

- l'extension du talonide plus large et aussi longue que le trigonide;

- l'aspect fragile de l'entoconide pointu et incliné distalement.

Cette suite de caractères se retrouve sur le matériel attribuable à Vespertiliavus kasserinensis Ravel, n. sp., témoignant ainsi de son statut d'Emballonuridae. D'après le registre fossile, la famille des Emballonuridae est documentée depuis l'Éocène moyen, et constitue de ce fait l'une des plus anciennes familles de chiroptère moderne. Tachypteron franzeni Storch, 2002, découvert dans le gisement de Messel (Éocène moyen; MP11), était considéré jusqu'à présent comme le plus ancien représentant de cette famille (par ex. : Storch et al. 2002; Habersetzer et al. 2012; Smith et al. 2012). Les conditions de fossilisation exceptionnelles qui caractérisent le site de Messel ont permis la préservation de squelettes complets de T. franzeni. Malheureusement, cette abondance de données, notamment sur la morphologie crânienne et post-crânienne, se fait parfois au détriment de la morphologie dentaire, qui n'est souvent pas directement accessible (vues occlusales notamment). Une description a été toutefois réalisée par Storch et al. (2002) sur la base de radiographies réalisées sur l'holotype. Vespertiliavus kasserinensis Ravel, n. sp. partage avec T. franzeni la conservation de trois prémolaires inférieures dont la taille est croissante de la partie mésiale vers la partie distale. Il s'en écarte toutefois par les caractères suivants:
- la présence d'un profond ectoflexus au niveau du bord buccal des M1-2;

- l'orientation distale de la postprotocrête qui rejoint l'hypocône ou le cingulum buccal;

- la communication de la protofosse avec le bassin du talon;

- l'orientation distale du talon;

- la présence d'un hypocône rudimentaire.

Si ces caractères, essentiellement situés sur les molaires supérieures, sont commun chez les Emballonuridae, ils écartent la possibilité que $V$. kasserinensis Ravel, n. sp. et T. franzeni appartiennent à un seul et même genre. Les divergences citées ci-dessus trouvent plutôt un écho au sein du genre Vespertiliavus.

Le genre Dhofarella Sigé, Thomas, Sen, Gheerbrant, Roger \& Al-Sulaimani, 1994 regroupe deux espèces d'Emballonuridae fossiles documentées dans l'Éocène supérieur du Fayum par la présence d'un fragment de mandibule portant $\mathrm{m} 1$ à $\mathrm{m} 3$ (D. sigei Gunnell, Simmons \& Seiffert, 2008, Quarry L-41, Égypte; Gunnell et al. 2008) et dans l'Oligocène inférieur de Taqah par quelques dents isolées ( $D$. thaleri Sigé, Thomas, Sen, Gheerbrant, Roger \& Al- Sulaimani, 1994, Sultanat d'Oman; Sigé et al. 1994). Concernant les molaires supérieures, Vespertiliavus kasserinensis Ravel, n. sp. differe significativement de D. thaleri par:

- l'absence d'un ectoflexus distal au mésostyle;

- la présence de la postprotocrête connectée au cingulum lingual (la postprotocrête est absente chez $D$. thaleri);

- la position plus buccale mais également plus mésiale du parastyle;

- le bassin du talon plus étendu;

- la formation d'un bourrelet distolingual;

- la présence de l'hypocône. 
Les molaires inférieures de Dhofarella présentent un talonide relativement moins large et moins long que celui de $V$. kasserinensis Ravel, n. sp. Hormis cette différence, les molaires inférieures des deux taxons sont assez semblables et typiques des Emballonuridae. Le genre Vespertiliavus est documenté par neuf espèces (deux sous-espèces sont considérées pour l'espèce $V$. disjunctus) retrouvées dans les poches phosphatées du Quercy datées depuis le milieu de l'Éocène moyen (Cuzal, MP13) jusqu'au début de l'Oligocène supérieur (Garouillas, MP25; Sudre 1979; Legendre 1980; Sigé 1990; Marandat et al. 1993; Maitre 2014). Le patron dentaire de Vespertiliavus présente toutes les caractéristiques anatomiques définissant les Emballonuridae. La nouvelle espèce de Chambi, $V$. kasserinensis Ravel, n. sp., ainsi que les formes européennes partagent également un ectoflexus mésial profond, une p3 réduite et étroite mais allongée et biradiculée. Cependant l'espèce africaine présente certaines divergences avec le reste de l'ensemble Vespertiliavus: - une $\mathrm{p} 2$ très réduite, moins longue que $\mathrm{p} 3$;

- un parastyle de la M1 désolidarisé de la préparacrête et ayant une position nettement plus linguale;

- un ectoflexus plus prononcé;

- un bourrelet distolingual au talon;

- un cingulum buccal de $\mathrm{C} 1$ mieux défini;

- une différence de hauteur entre la protofosse et le bassin du talon plus réduite;

- un cingulum buccal plus épais.

\section{?Vespertiliavus aenigma Ravel, n. sp.} (Figs 14, 15; Tableau 5)

Holotype. - CBI-2-169, M1 droite, Figs 14D; 15E.

MATÉRIEl eXAMiné. - CBI-2-175 (C1 droite; Figs 14A; 15A); CBI-2-176 (Figs 14B; 15B) et CBI-2-177 (C1s gauches; Fig. 15C); CBI-2-174 (P4 gauche; Figs 14C; 15D); CBI-2-170 (M1 gauche; Figs 14E; 15F); CBI-2-171 (ectolophe M2 droite; Figs 14F; 15G); CBI-2-173 (M3 gauche) ; CBI-3-013 (Figs 14G ; 15I) et CBI-2-172 (M3s? gauches; Fig. 15H); CBI-2-205 (c1 droite; Figs 14H; 15J); CBI-2-183 (p4 droite; Figs 14J; 15K); CBI-2-182 (p4 gauche; Figs 14I ; 15L) ; CBI-2-178 (Figs 14L; 15P), CBI-2-179 (Fig. 15N), CBI-2-181 (Figs $14 \mathrm{~K} ; 15 \mathrm{M}$ ) et CBI-1-262 (m1/2s droites); CBI2-180 (m1/2 gauche; Fig. 15O).

ÉTYMOLOGIE. - Du latin «aenigma» qui signifie énigme, en raison de l'originalité des morphologies caractérisant ces spécimens et rendant ainsi l'attribution systématique difficile.

LoCAlité TYPE ET ÂGE. - Chambi (CBI) loci 1, 2 et 3, Éocène inférieur terminal-Éocène moyen basal, Djebel Chambi, situé dans la région de Kasserine, Tunisie.

DiAGNOSE. - Vespertiliavus caractérisé par une canine supérieure avec un cingulum très épais et continu, une $\mathrm{P} 4$ très étirée longitudinalement, et une M3 possédant un ectolophe qui conserve la postmétacrête et le métastyle semblable au M1/2. Espèce plus grande (environ une fois et demi) que Vespertiliavus kasserinensis Ravel, n. sp.

\section{DESCRIPTION}

Le matériel attribuable à cette plus grande espèce du genre Vespertilavus est très fragmentaire. Les canines supérieures ne possèdent qu'un seul tubercule de hauteur modérée et légère- ment recourbé vers la partie distale (Figs 14A, B; 15A-C). Le cône est constitué d'une face buccale convexe et d'une face linguale légèrement concave. Les deux faces sont délimitées par une crête mésiale et une crête distale plus affutée. Le cingulum, très épais et continu, porte deux bourrelets à la base linguale de la couronne.

La P4 est très étirée longitudinalement mais comprimée buccolingualement (Figs 14C; 15D). La couronne possède un contour triangulaire en vue occlusale dont le côté linguodistal est fortement incurvé vers l'intérieur. La dent est munie d'un unique paracône positionné centrobuccalement. Une crête saillante longiligne (i.e., postparacrête) s'étend depuis le sommet du paracône pour rejoindre le bord buccodistal de la couronne. Cette crête prend une orientation à prédominance distale, et s'incurve très légèrement vers le bord buccal. Le bassin lingual est comprimé transversalement et s'étale sur la moitié mésiolinguale de la couronne. Un fin cingulum lingual cerne le bord lingual du bassin.

La couronne de la M1 CBI-2-169 possède un talon très projeté distalement (Figs 14D; 15E). Le bord buccal est incliné mésiolingualement et présente deux ectoflexus de part et d'autre de la région mésostylaire. Le parastyle est légèrement plus lingual que le mésostyle, et nettement plus par rapport au métastyle. Le parastyle est modérément recourbé vers le côté mésial pour former un crochet antérieur. Le métastyle est simple et apparaît buccalement en continuité avec la postmétacrête. Le cingulum buccal, discontinu, est toutefois très épais au niveau du mésostyle. Le mésostyle, situé à la jonction entre la postparacrête et la prémétacrête, est légèrement déplacé lingualement par rapport au bord buccal. Les crêtes de l'ectolophe sont assez divergentes et disposées en "W» ouvert mais légèrement compressé buccolingualement. Le paracône est peu réduit et plus lingual que le métacône. Ces deux tubercules sont séparés par une large et longue protofosse qui est délimitée buccalement par le mésostyle. Le protocône est buccolingualement compressé. Il occupe une position très mésiolinguale tout en restant un peu moins mésial que le paracône. Le protocône est plus comprimé buccolingualement que le métacône et le paracône. La préprotocrête remonte buccalement depuis le sommet du protocône pour rejoindre le précingulum, clôturant ainsi la protofosse mésialement. La postprotocrête est deux fois plus longue que la préprotocrête, et beaucoup plus rectiligne, avec une direction distolinguale depuis le sommet du protocône. Cette crête se termine distalement au niveau d'une triple jonction réalisée avec le cingulum lingual et une crête tranchante qui s'étend sur presque toute la largeur buccolinguale de la couronne depuis le postcingulum. Cette dernière crête marque la limite entre la protofosse et le bassin du talon. Ce dernier est circulaire et se déploie distalement, formant ainsi une structure fortement dissociée du reste de la couronne mais qui participe à une large et longue surface d'occlusion. Son extrémité distale est circulaire et ceinturée par un cingulum lingual modérément épais.

CBI-2-170 (Figs 14E; 15F) est interprétée ici comme une M1 de par sa taille, laquelle est similaire à celle de CBI-2-169. $\mathrm{La}$ forte inclinaison du bord buccal, la position linguale du parastyle, la projection distale du talon sont des caractères 


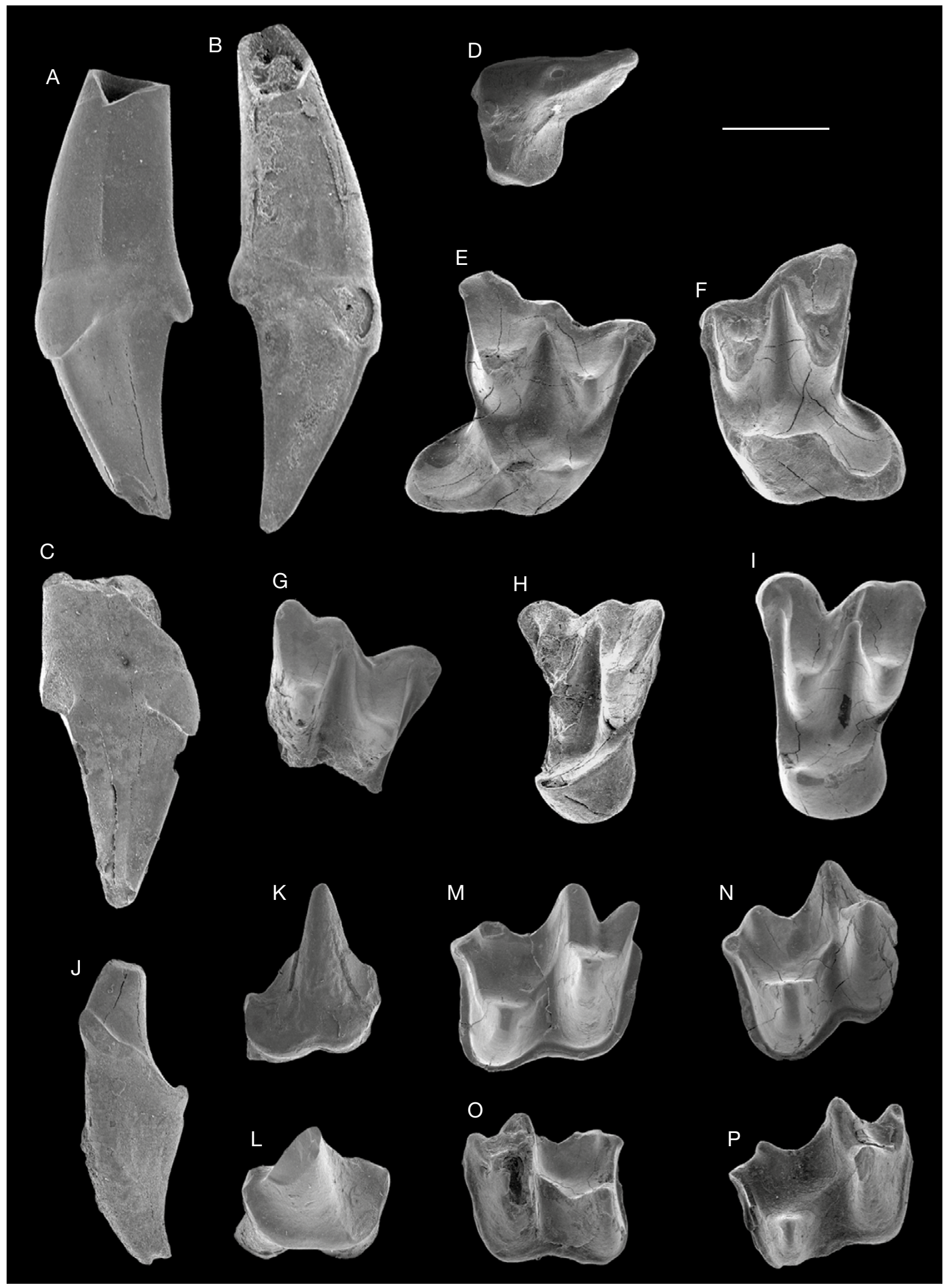

FIG. 15. - ?Vespertiliavus aenigma Ravel, n. sp. provenant des loci 2 et 3 du Djebel Chambi dans la région de Kasserine, Tunisie: A, CBI-2-175, C1 droite en vue linguale; B, CBI-2-176, C1 gauche en vue linguale; C, CBI-2-177, C1 gauche en vue linguale; D, CBI-2-174, P4 gauche en vue occlusale; E, CBI-2-169, M1 droite en vue occlusale; F, CBI-2-170, M1-2 gauche en vue occlusale; G, CBI-2-171, ectolophe de M2 droite en vue occlusale; H, CBI-2-172, M3? gauche en vue occlusale; I, CBI-3-013, M3? gauche en vue occlusale; J, CBI-2-205, c1 droite en vue linguale; K, CBI-2-183, p4 droite en vue buccale; L, CBI-2-182, p4 gauche en vue semi buccale; $\mathbf{M}, \mathrm{CBI}-2-181, \mathrm{~m} 1 / 2$ droite en vue occlusale; $\mathbf{N}, \mathrm{CBI}-2-179, \mathrm{~m} 1 / 2$ droite en vue occlusale; $\mathbf{O}, \mathrm{CBI}-2-180, \mathrm{~m} 1 / 2$ gauche en vue occlusale; P, CBI-2-178, m1/2 droite en vue occlusale. Échelle: $1 \mathrm{~mm}$. 
comparables à l'holotype (CBI-2-169, M1; Figs 14D; 15E). Cependant ces deux dents different dans l'agencement et la morphologie de certaines structures. Sur CBI-2-170, la bordure buccale est plus courte et présente un seul ectoflexus, lequel est moins prononcé que sur CBI-2-169. Sur CBI-2-170, la région parastylaire est brisée, ce qui ne permet pas d'évaluer sa morphologie ni sa projection. Distobuccalement, l'ectolophe est plus resserré mésiodistalement, générant ainsi une parafosse et une métafosse plus étendues buccolingualement mais plus compressées mésiodistalement. La postprotocrête est plus courte. Elle rejoint le cingulum lingual, laissant ainsi une protofosse ouverte distalement, qui communique ainsi avec le bassin du talon. Le cingulum lingual apparaît également plus épais. Ces différences peuvent s'expliquer soit par une variation intraspécifique - courante au sein des Vespertiliavus (Maitre 2014) - soit par la présence ici d'une espèce différente.

Un fragment d'ectolophe d'une M2 (Fig. 14F; 15G) possède une taille et une morphologie qui pourrait correspondre à ?V. aenigma Ravel, n. sp. Ce dernier possède un bord buccal très légèrement incliné mésiolingualement qui indique une M2 plutôt qu'une M1. Il présente un très léger ectoflexus au dessus du métacône et un autre nettement plus prononcé qui surplombe le paracône. Le parastyle est modérément recourbé et projeté vers bord mésial.

Les M3s? (Figs 14G; 15H, I) ont une largeur équivalente à celle des M1s. L'ectolophe est complet avec un fort ectoflexus au niveau du paracône. À la différence du métastyle qui apparaît comme un simple et petit style, le parastyle forme un crochet buccomésial. Le paracône est plus pincé et plus lingual que le métacône. Le métacône et légèrement réduit. Les post- et préprotocrêtes rejoignent respectivement les pré- et postcinginguli, délimitant ainsi mésialement et distalement la protofosse. Le protocône est réduit et présente un flanc lingual arrondi dépourvu de cingulum. La morphologie de cette dent est peu commune chez les chiroptères. Cependant l'absence de cingulum lingual ne peut correspondre avec une $\mathrm{M} 1$ ou une M2 (condition généralisée chez les chiroptères). Le type M3 semble le plus probable. En terme de taille, le matériel référé à ?Vespertiliavus aenigma Ravel, n. sp. semble le plus adéquat. Le fort développement de la M3 chez les Emballonuridae en général aurait pu être accentué chez ce taxon. En absence de matériel plus fourni, une telle association reste cependant en suspend.

La canine inférieure est allongée et relativement étroite. La partie la plus apicale de la racine possède une longueur équivalente à la couronne (Figs $14 \mathrm{H} ; 15 \mathrm{~J}$ ). Cette dernière est constituée d'un unique tubercule (cassé au quart de sa hauteur sur CBI-2-205) présentant une section ovale. Le cingulide n'est visible que sur la face linguale, il est absent au niveau de la base buccale du conide. Le cingulum est fortement incliné distalement. Il effectue un ressaut au niveau de la bordure distale, faisant naître un petit relief qui délimite le bassin de la canine.

La couronne de la $\mathrm{p} 4$ présente un contour rectangulaire étroit mais allongé en vue occlusale (Figs 14I, J; 15K, L). Sa longueur équivaut à la moitié de celle de m1-2. Elle possède un unique protoconide, très haut et pointu, qui domine la partie mésiale. Une courte crête débute au quart de la hauteur de cette cuspide et s'oriente mésiolingualement, formant ainsi un relief prononcé à l'avant de la couronne. La partie distale est composée d'un bassin modérément allongé mais occupant toute la largeur de la couronne. Une crête distale apparaît depuis le quart de la hauteur du protoconide et rejoint le bord distal délimitant la bordure linguale du bassin du talonide. Un cingulide fin et continu parcourt tout le contour de la dent.

Le trigonide des molaires inférieures est plus court et nettement plus étroit que le talonide (Figs $14 \mathrm{~K}, \mathrm{~L} ; 15 \mathrm{M}, \mathrm{N}, \mathrm{O}$, $\mathrm{P})$. Le paraconide, plus petit que le métaconide, est proclive. Le protoconide, légèrement compressé mésiodistalement, constitue la cuspide la plus volumineuse de la dent. La crête oblique débute depuis la base de la muraille distale du trigonide, au niveau de l'échancrure entre le métaconide et le protoconide. Elle rejoint l'hypoconide (beaucoup plus buccal que le protoconide) en effectuant une forte inflexion buccale. L'hypoconide est faiblement pincé mésiodistalement à son extrémité. La postcristide se projette distolingualement depuis le sommet de l'hypoconide jusqu'à l'hypoconulide, définissant ainsi une structure nyctalodonte. L'hypoconulide est très distal et se situe légèrement plus lingualement que l'entoconide, ce dernier étant élevé et saillant. L'entocristide forme une muraille linguale au bassin du talonide en débutant depuis le sommet de l'entoconide et rejoignant le tiers de la hauteur distale du métaconide. Un fin cingulide est ininterrompu le long des bordures mésiale, buccale et distale de la couronne.

\section{COMPARAISON ET DISCUSSION}

Les spécimens décrits ci-dessus présentent une grande variation de forme qui pourrait éventuellement refléter ici la présence de plusieurs espèces. Toutefois, le matériel présente un ensemble de caractères dentaires assez commun chez les Emballonuridae, et plus particulièrement chez le genre fossile Vespertiliavus. Ces caractères sont:

- le cingulum des canines supérieures bien individualisé;

- la compression buccolinguale des P4 et leur contour occlusal triangulaire;

- le parastyle des M1 déplacé lingualement, entraînant une inclinaison modérée à forte du bord buccal ;

- le bassin du talon très étendu distalement et en largeur;

- la postprotocrête orientée distalement rejoignant parfois l'hypocône ou le cingulum lingual;

- la connexion entre la protofosse et le bassin du talon, conférant à la première et la seconde molaire une large surface d'occlusion (pour CBI-2-170; Figs 14E; 15F);

- p4 à contour rectangulaire étroit mais allongé;

- le protoconide de $\mathrm{p} 4$ très haut et pointu;

- la compression mesiodistale du trigonide formant un « $V$ » refermé en vue occlusale;

- l'aspect gracile de l'entoconide, lequel est pointu et incliné distalement;

- l'extension du talonide plus large que le trigonide et aussi long;

- la M3 bien développée, munie d'un métacône bien défini.

La morphologie et l'orientation de la postprotocrête ainsi que l'isolement du bassin du talon (observées sur CBI-2-169, l'holotype; Fig. 14D; 15E), représente un agencement peu 
TABLEAU 6. - Mesures dentaires (en mm) de Pseudovespertiliavus parva Ravel n. gen., n. sp. provenant des niveaux HGL50 et HGL50' du Glib Zegdou situé dans les Gour Lazib, Algérie. Abréviations: L, longueur; I, largeur; H, hauteur; Ltdr, longueur du trigonide; Itdr, largeur du trigonide; Ltdl, longueur du talonide; Itdl, largeur du talonide. Le signe * derrière une valeur indique un biais dans la mesure causé par l'altération de la couronne.

\begin{tabular}{|c|c|c|c|c|c|c|c|c|c|c|}
\hline Spécimen & Localité & $\begin{array}{l}\text { Type de } \\
\text { matériel }\end{array}$ & $\mathbf{L}$ & $\mathbf{I}$ & $\mathbf{H}$ & Ltrd & Itrd & Ltld & Itld & Remarques \\
\hline UM/HGL50-433 & HGL50, Glib Zegdou & C1 droite & 0,83 & 0,63 & 1,25 & - & - & - & - & - \\
\hline UM/HGL50-431 & HGL50, Glib Zegdou & C1 gauche & 0,85 & 0,62 & $1,04^{*}$ & - & - & - & - & - \\
\hline UM/HGL50-432 & HGL50, Glib Zegdou & C1 gauche & 0,89 & 0,74 & $1,24^{*}$ & - & - & - & - & apex cassé \\
\hline UM/HGL50-429 & HGL50, Glib Zegdou & P3? Gauche & 0,64 & 0,58 & - & - & - & - & - & apex cassé \\
\hline UM/HGL50-430 & HGL50, Glib Zegdou & P3? Droite & - & $0,73^{*}$ & - & - & - & - & - & partie buccale cassée \\
\hline UM/HGL50-423 & HGL50, Glib Zegdou & M1 droite & $1,13^{*}$ & 1,31 & - & - & - & - & - & parastyle manquant \\
\hline UM/HGL50-427 & HGL50, Glib Zegdou & M1 droite & 1,37 & 1,55 & - & - & - & - & - & - \\
\hline UM/HGL50-420 & HGL50, Glib Zegdou & M1 gauche & 1,42 & 1,38 & - & - & - & - & - & - \\
\hline UM/HGL50-424 & HGL50, Glib Zegdou & M1 gauche & $1,24^{*}$ & 1,47 & - & - & - & - & - & talon manquant \\
\hline UM/HGL50'-58 & HGL50', Glib Zegdou & M1 gauche & - & - & - & - & - & - & - & - \\
\hline UM/HGL50-422 & HGL50, Glib Zegdou & M2 droite & 1,17 & 1,45 & - & - & - & - & - & - \\
\hline UM/HGL50-425 & HGL50, Glib Zegdou & M2 droite & 1,06 & 1,34 & - & - & - & - & - & - \\
\hline UM/HGL50-428 & HGL50, Glib Zegdou & M2 droite & $1,49^{*}$ & $0,98^{*}$ & - & - & - & - & - & ectolophe uniquement \\
\hline UM/HGL50-421 & HGL50, Glib Zegdou & M2 gauche & 1,39 & 1,63 & - & - & - & - & - & - \\
\hline UM/HGL50-426 & HGL50, Glib Zegdou & M1/2 gauche & - & - & - & - & - & - & - & - \\
\hline UM/HGL50-435 & HGL50, Glib Zegdou & m1-2 droite & 1,08 & 0,71 & - & 0,53 & 0,69 & 0,55 & 0,71 & - \\
\hline UM/HGL50-439 & HGL50, Glib Zegdou & m1-2 droite & $1,04^{*}$ & $0,73^{*}$ & - & - & - & - & - & $\begin{array}{l}\text { dent très fracturée et } \\
\text { recollée }\end{array}$ \\
\hline UM/HGL50'-59 & HGL50', Glib Zegdou & m1/2 droite & - & - & - & 0,47 & 0,66 & - & - & trigonide uniquement \\
\hline UM/HGL50-434 & HGL50, Glib Zegdou & $\mathrm{m} 1 / 2$ gauche & 1,16 & 0,75 & - & 0,56 & 0,7 & 0,6 & 0,75 & - \\
\hline UM/HGL50-436 & HGL50, Glib Zegdou & $\mathrm{m} 1 / 2$ gauche & 1,14 & 0,73 & - & 0,52 & 0,69 & 0,62 & 0,73 & - \\
\hline UM/HGL50-438 & HGL50, Glib Zegdou & $\mathrm{m} 1 / 2$ gauche & - & - & - & - & - & - & - & $\begin{array}{l}\text { couronne très érodée, } \\
\text { structures non } \\
\text { reconnaissables car } \\
\text { trop émoussées }\end{array}$ \\
\hline UM/HGL50-440 & HGL50, Glib Zegdou & $\mathrm{m} 1 / 2$ gauche & - & - & - & - & - & 0,6 & 0,68 & - \\
\hline UM/HGL50-437 & HGL50, Glib Zegdou & m3 droite & 0,94 & 0,63 & - & 0,47 & 0,63 & 0,47 & 0,51 & - \\
\hline UM/HGL50-441 & HGL50, Glib Zegdou & m3 droite & 1 & 0,6 & - & 0,5 & 0,6 & 0,5 & 0,47 & - \\
\hline
\end{tabular}

commun au sein des Emballonuridae. Parmi le matériel de comparaison, seul Saccolaimus peli Temminck, 1853 présente un bassin isolé (uniquement sur la M1), mais cet isolement est réalisé par l'extension du métalophe qui rejoint la postprotocrête, et n'implique donc pas les mêmes structures. Dans l'abondant matériel du Quercy attribuable au genre Vespertiliavus (collection hébergé à l'ISE-M, matériel provenant des niveaux repères MP13 à MP26), certains spécimens présentent une courte postprotocrête orientée distobuccalement, entraînant une rupture avec le cingulum lingual. Cette variation, que l'on retrouve au sein d'une espèce ( $V$. bourguignati Filhol, 1877; La Bouffie MP17a), n'aboutit cependant jamais à une fermeture franche de la protofosse (la postprotocrête s'interrompt avant de rejoindre la base du métacône). Sur certains aspects, notamment au niveau de l'ectolophe, CBI-2-169 se rapproche des Mixopterygidae et plus précisément du genre Mixopteryx Maitre, Sigé \& Escarguel, 2008 (Maitre et al. 2008). La particularité des Mixopterygidae est de combiner des caractères emballonuroïdes avec des caractères rhinolophoïdes. Ces caractéristiques n'ont pas permis de rapprocher cette famille de l'un des grands ensembles de chiroptères actuels. CBI-2-169 possède un double ectoflexus, un mesostyle bien projeté buccalement, et un parastyle déjeté mésialement. Cette suite de caractères s'observe également chez Mixopteryx. Cependant, les molaires inférieures de ? Vespertiliavus aenigma Ravel, n. sp. diffèrent radicalement des rhinolophoïdes et présente un patron dentaire typique des Emballonuridae. En effet, chez les emballonuroïdes, tout comme chez ?V. aenigma Ravel, n. sp., le trigonide est fermé, l'entoconide est haut et penché distalement, et le talonide est plus large que le trigonide. En revanche, chez les rhinolophoïdes, et notamment chez les Mixopterygidae, les molaires inférieures ont un trigonide ouvert, l'entoconide est peu élevé et arrondi, le talonide est réduit, et les cuspides sont larges.

L'autre fait remarquable relatif à ? $V$. aenigma Ravel, n. sp. est la morphologie de ses M3s. Elles ont une bordure linguale arrondie et dépourvue de cingulum. Cependant la morphologie et la taille de l'ectolophe de ces dents sont similaires aux conditions observées sur les M1-2s. Ces similitudes incluent des critères morphologiques attribuables à un Emballonuridae: ectoflexus profond au niveau du paracône, resserrement des crêtes de l'ectolophe avec un développement transverse de ce dernier, cingulum buccal bien développé, protofosse étendue, et protocône déjeté vers l'avant. Même si les M3s de la majorité des espèces d'Emballonuridae actuels (par ex. : Peropteryx macrotis Wagner, 1843, Rhynchiscus naso Maximilian, 1820, Rhynchonycteris naso Maximilian, 1820, Saccopteryx leptura Schreber, 1774 et S. bilineata Temminck, 1838, Emballonura atrata Peters, 1874, Diclidurus albus Wied-Neuwied, 1820, et Cormura brevirostris Wagner, 1843) ne sont pas aussi complexes, il existe des cas où le métacône peut-être bien individualisé, avec une largeur de dent similaire à celle des M2s. Ces caractéristiques sont également observables chez les formes fossiles Tachypteron franzeni et l'ensemble des Vespertiliavus. Il est donc possible que ce morphotype caractérise l'espèce ?Vespertiliavus aenigma Ravel, n. sp. 

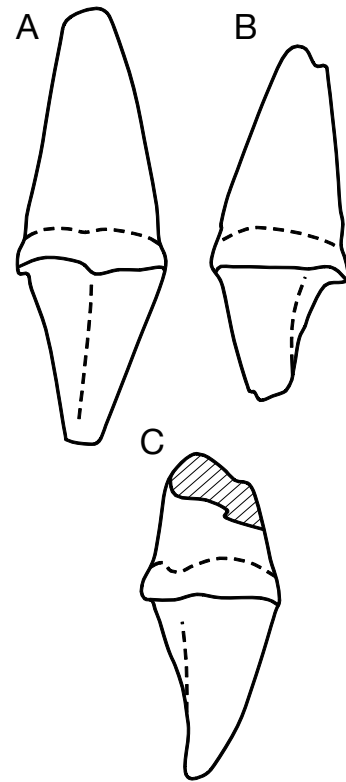
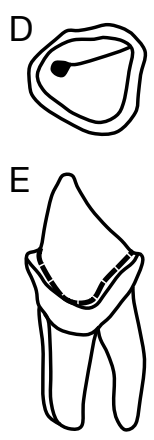

$\mathrm{F}$
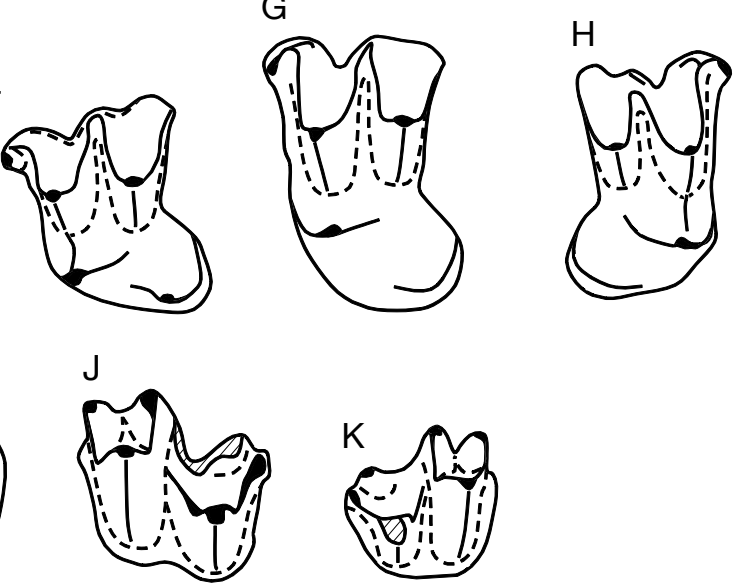

FIG. 16. - Pseudovespertiliavus parva Ravel n. gen., n. sp. provenant du niveau HGL50 et HGL50' du Glib Zegdou situé dans les Gour Lazib, Algérie: A, UM/ HGL50-432, C1 droite en vue linguale; B, UM/HGL50-431, C1 gauche en vue buccale; C, UM/HGL50-433, C1 droite en vue buccale; D, UM/HGL50-429, P3? gauche en vue occlusale; E, UM/HGL50-429, P3? gauche en vue linguale; F, UM/HGL50-420, M1 gauche en vue occlusale; G, UM/HGL50-421, M2 gauche en vue occlusale; H, UM/HGL50-422, M2 droite en vue occlusale; I, UM/HGL50-435, m1/2 droite en vue occlusale; J, UM/HGL50-434, m1/2 gauche en vue occlusale; K, UM/HGL50-437, m3 droite en vue occlusale. Échelle: $1 \mathrm{~mm}$.

Genre Pseudovespertiliavus Ravel, n. gen.

ESPÈCE TYPE. - Pseudovespertiliavus parva Ravel, n. sp.

ÉTYMOLOGIE. - Le nom de genre fait référence aux nombreuses similitudes morphologiques observées avec le genre Vespertiliavus.

Diagnose. — Comme celle de l'espèce type.

Pseudovespertiliavus parva Ravel, n. sp. (Figs 16, 17; Tableau 6)

HolOTYPE. — UM/HGL50-420, M1 gauche (Figs 16D; 17D).

MatéRIEL EXAMINÉ. - UM/HGL50-432 (Figs 16A; 17A) et UM/HGL50-433 (C1 droite; Figs 16C; 17C) ; UM/HGL50-431 (C1 gauche; Figs 16B; 17B); UM/HGL50-430 (P3? Droite); UM/HGL50-429 (P3? Gauche; Fig. 16D, E) ; UM/HGL50-423 (Fig. 17G) et UM/HGL50-427 (M1s droites); UM/HGL50-420 (Figs 16F; 17D), UM/HGL50-424 (Fig. 17I) et UM/HGL50'058 (M1s gauches) ; UM/HGL50-422 (Figs 16H; 17F), UM/ HGL50-425 (Fig. 17H) et UM/HGL50-428 (M2s droites); UM/HGL50-421 (M2 gauche; Figs 16G; 17E) UM/HGL50426 (M1/2 gauche); UM/HGL50-435 (Figs 16I ; 17L) et UM/ HGL50-439 (m1/2s droites); UM/HGL50-434 (Figs 16J ; 17K), UM/HGL50-436 (Fig. 17J), UM/HGL50-438 et UM/HGL50440 (m1/2s gauches); UM/HGL50'-059 (trigonide de $\mathrm{m} 1 / 2$ droite) ; UM/HGL50-437 (Figs 16K; 17M) et UM/HGL50-441 (m3s droites).

ÉTYMOLOGIE. — le nom d'espèce est issu du latin «parva » qui signifie petit, en raison de la petite taille de cette espèce de chauve-souris.

LOCAlité TYPE ET ÂGE. - Niveaux HGL50 et HGL50' du Glib Zegdou, Éocène inférieur terminal-Éocène moyen basal, situé dans la région des Gour Lazib (Sahara, Hammada du Dra) en Algérie.
DiAgNOSE. - Forme emballonuride de très petite taille, caractérisée par le cingulum des canines supérieures quasi rectiligne, épais et continu, la P3 à trois racines, l'ectoflexus mésial profond, la postprotocrête longue mais non connectée au cingulum lingual, le paralophe long rejoignant parfois le protocône, le talon projeté linguodistalement, le cingulum lingual d'épaisseur modérée connecté à un hypocône très réduit; molaires inférieures nyctalodontes caractérisées par le trigonide étroit, l'entoconide moyen incliné distalement, les $\mathrm{m} 3$ avec les trois cuspides du talonide bien développées.

\section{DESCRIPTION}

La couronne de la canine supérieure est dominée par une seule cuspide. Ce dernier est incliné distalement au niveau de son apex. La face buccale convexe de la cuspide est la plus étendue et s'oppose à la face linguale plane plus réduite. Ces deux faces sont délimitées par une crête très fine mais haute et d'orientation linguodistale, et par une autre crête moins prononcée et orientée linguomésialement. Le cingulum est épais et continu sur tout le pourtour de la couronne. Cette structure présente parfois un léger ressaut distal qui est rejoint par la crête distale (visible sur UM/ HGL50-431 et UM/HGL50-432; Figs 16A, B ; 17A, B).

L'absence d'un fragment de maxillaire pouvant être associée à ce taxon ne permet pas d'évaluer la composition de la rangée dentaire. Cependant deux spécimens (UM/ HGL50-429 et UM/HGL50-430) de taille très petite ( $<1 \mathrm{~mm}$ de long et de large) et dont la couronne est simplifiée pourraient être des P3 (Fig. 16D, E). Ces dents sont munies de trois racines (deux en position buccale et une en position linguale), ce qui écarte toute possibilité que ce soient des prémolaires inférieures. La couronne prend une forme triangulaire en vue occlusale. La présence d'un 


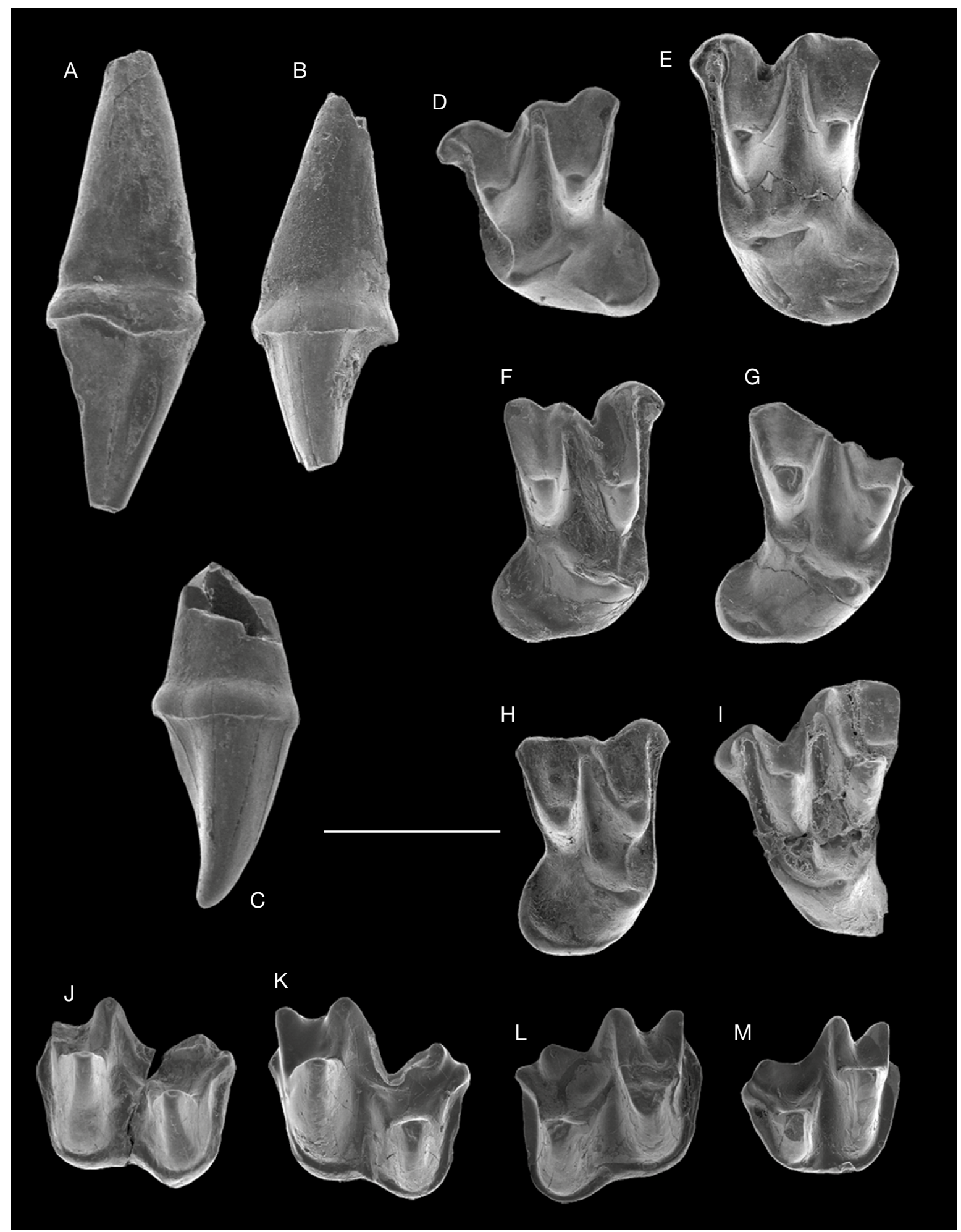

FIG. 17. - Pseudovespertiliavus parva Ravel n. gen., n. sp. provenant du niveau HGL50 du Glib Zegdou situé dans les Gour Lazib, Algérie: A.UM/HGL50-432, C1 droite en vue linguale; B, UM/HGL50-431, C1 gauche en vue buccale; C, UM/HGL50-433, C1 droite en vue buccale; D, UM/HGL50-420, M1 gauche en vue occlusale; E, UM/HGL50-421, M2 gauche en vue occlusale; F, UM/HGL50-422, M2 droite en vue occlusale; G, UM/HGL50-423, M1 droite en vue occlusale; H, UM/HGL50-425, M2 droite en vue occlusale; I, UM/HGL50-424, M1 gauche en vue occlusale; J, UM/HGL50-436, m1/2 gauche en vue occlusale; K, UM/ HGL50-434, m1/2 gauche en vue occlusale; L, UM/HGL50-435, m1/2 droite en vue occlusale; M, UM/HGL50-437, m3 droite en vue occlusale. Échelle: 1 mm.

fort cingulum encerclant l'ensemble de la couronne plaide pour leur appartenance aux chiroptères. L'unique tubercule est en position buccale. Une haute crête s'étend depuis l'apex de la cuspide pour rejoindre l'extrémité distale de la couronne tandis qu'une autre crête plus courte prend une orientation linguale. La face buccale bombée de la petite 
cuspide est opposée à une face linguale concave qui s'étend vers un petit bassin distolingual.

M1 possède un large et long talon lui conférant un fort développement longitudinal. Le bord buccal, incliné mésiolingualement, est creusé par deux ectoflexus dont le plus profond se situe mésialement au mésostyle. Le parastyle forme une petite cuspide désolidarisé de la préparacrête. Elle se positionne plus lingualement par rapport au mésostyle et au métastyle. Le mésostyle est fortement projeté buccalement mais reste toutefois plus lingual que le métastyle. La forte projection du mésostyle s'accompagne d'une extension buccale et prononcée de la protofosse. Le métastyle constitue la terminaison distobuccale de la postmétacrête. L'orientation des crêtes confère à l'ectolophe une forme en "W" assez ouvert mais dissymétrique: le « $\mathrm{V}$ » antérieur formé des pré- et postparacrêtes est légèrement plus ouvert mais comprimé buccolingualement que le « $\mathrm{V}$ » postérieur formé des pré- et postmétacrêtes. Le paracône est plus lingual et légèrement moins volumineux que le métacône. Le paralophe est présent et très accentué sur la plupart des molaires (seul UM/HGL50-423 n'en possède pas). Le paralophe débute depuis la base du paracône et s'oriente en direction du protocône. Sur certaines molaires (UM/ HGL50-420, UM/HGL50-424, et UM/HGL50-427; Figs $16 \mathrm{~F} ; 17 \mathrm{D}, \mathrm{I})$, il rejoint le protocône créant une petite fosse mésiale qui prolonge le large précingulum. Le protocône est déjeté mésialement mais il reste plus distal que le paracône. La préprotocrête se projette vers la partie buccomésiale et se connecte au précingulum. La postprotocrête présente une orientation plus variée. Dans la majorité des cas, elle ne rejoint aucune autre structure, ce qui laisse une ouverture distale plus ou moins large à la protofosse. D'autres molaires (UM/HGL50-423 et UM/ HGL50-424; Figs 17G, I) montrent une postprotocrête directement connectée au postcingulum cernant distalement la protofosse. Le talon s'étend selon une direction linguodistale. Sur UM/HGL50-420, il effectue un léger renflement linguodistal alors que la bordure du talon est plus arrondie sur les autres molaires. Le talon s'aplanit dans sa partie la plus distale prenant la forme d'un large bassin. Le bassin du talon est cerné par un cingulum d'épaisseur modérée. UM/HGL50-420 possède une ébauche d'hypocône réalisée par le développement du cingulum lingual. Cette ébauche est moins aboutie sur les autres molaires qui présentent toutefois, à cette place, un léger relief.

M2 diffère de M1 par une taille nettement plus grande, un développement transversal plus accentué, notamment au niveau de l'ectolophe (Figs 16G, H; 17E, F, H). Le bord buccal est très peu incliné. La dissymétrie des ectoflexus est encore plus prononcée avec un ectoflexus distal à peine visible qui contraste avec l'ectoflexus mésial très profond. Le protocône possède un flanc lingual plus élancé lui conférant un aspect moins comprimé buccolingualement. Le talon est également moins projeté distalement et le cingulum lingual paraît plus fin.
Les molaires inférieures se caractérisent par un trigonide étroit formant un triangle comprimé mésiodistalement en vue occlusale (Figs 16I, J; 17J, K, L). Les trois cuspides du trigonide sont bien individualisées. Le métaconide est légèrement plus haut et volumineux que le paraconide. Le protoconide domine les autres cuspides en volume et en hauteur. Son flanc lingual est pincé mésiodistalement. Le talonide est significativement plus large que le trigonide. Sa largeur est accentuée par la projection buccale de l'hypoconide. Ce dernier est également pincé mésiodistalement comme le protoconide. L'hypoconide reste toutefois moins volumineux et moins élevé que le protoconide. La cristide oblique est très longue et droite. Elle débute depuis le sommet de l'hypoconide et rejoint la base de la muraille distale du trigonide à un niveau plus lingual que la jonction entre la postprotocristide et la métacristide. L'entoconide est modérément élevé et déjeté distalement. Il est rejoint par une entocristide rectiligne en vue occlusale. Le talonide montre une structure nyctalodonte: la postcristide fait la jonction entre l'hypoconide et un petit hypoconulide. L'hypoconulide, incliné distalement, est positionné sur le même axe mésiodistal que l'entoconide tout en étant assez éloigné de celui-ci. Le cingulide, d'épaisseur moyenne, est continu sur les bordures mésiale, buccale et distale de la couronne. Il devient cependant très fin à la base linguomésiale du protoconide sur les spécimens UM/HGL50-434 et UM/HGL50-436.

Les $\mathrm{m} 3 \mathrm{~s}$ diffèrent des $\mathrm{m} 1 \mathrm{~s}$ et $\mathrm{m} 2 \mathrm{~s}$ par une taille plus réduite et une réduction modérée du talonide (Figs 16K; $17 \mathrm{M})$. Le trigonide de ce type de molaire est très étroit mais sa morphologie reste semblable à celle des m1-2s. La réduction du talonide se fait surtout au niveau de son extension buccolinguale et mésiodistale. Les structures sont bien différenciées avec des crêtes et des cuspides bien identifiables. L'hypoconulide est toutefois légèrement déplacé buccalement par rapport à l'entoconide, ce qui diffère de la position plus linguale de l'hypoconulide sur m1-2. Le cingulide est également continu avec toutefois une largeur plus accentuée sur la bordure mésiale.

\section{COMPARAISON ET DISCUSSION}

Le taxon décrit ci-dessus présente une liste de caractères que l'on retrouve de manière courante chez les espèces du genre Vespertiliavus et de manière plus générale chez les Emballonuridae:

- le cingulum épais des canines supérieures;

- le relief distal initié par le cingulum sur C1;

- la différence significative entre la M1 et la M2;

- l'amorce d'un hypocône;

- le talon des M1s et M2s très étendu distolingualement et constitué d'un large bassin;

- l'ouverture distale de la protofosse;

- la protofosse étendue notamment dans le sens buccolingual;

- la compression mésiodistale du trigonide;

- le talonide plus large et long que le trigonide;

- l'entoconide déjeté distalement. 

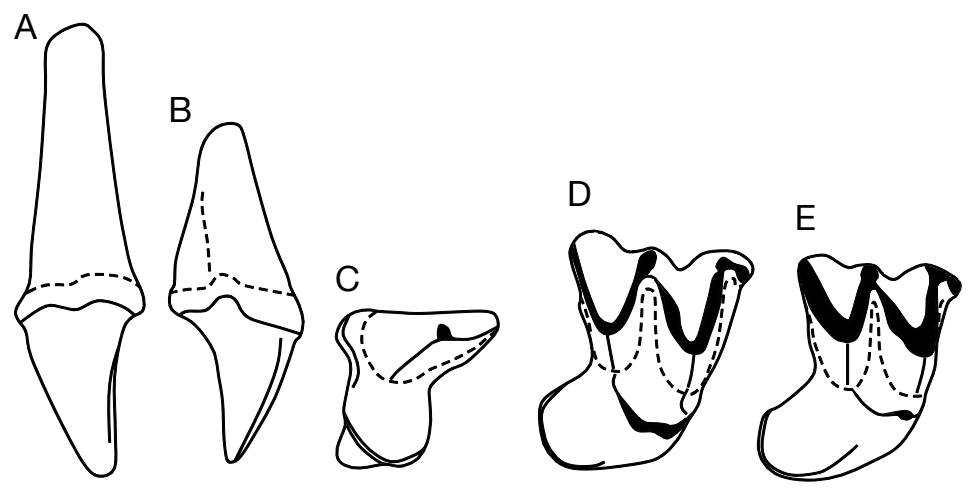

F

G

$\mathrm{H}$
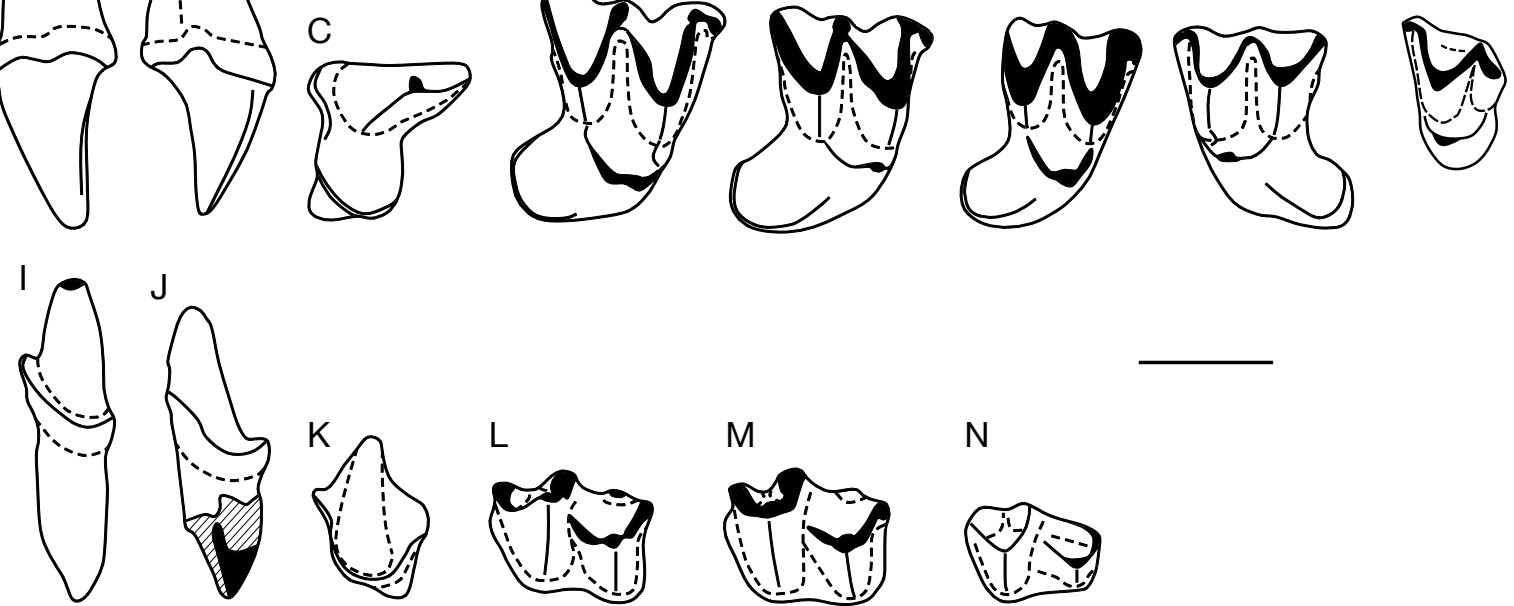

FIG. 18. - Khoufechia gunnelli Ravel n. gen., n. sp. provenant des localités 1 et 2 du Djebel Chambi dans la région de Kasserine, Tunisie: A, CBI-1-257, C1 droite en vue linguale; B, CBI-2-124, C1 gauche en vue linguale; C, CBI-2-120, P4 gauche en vue occlusale; $\mathbf{D}, \mathrm{CBI}-2-113, \mathrm{M} 2$ droite en vue occlusale; $\mathbf{E}$, CBI-2-114, M1 droite en vue occlusale; F, CBI-2-116, M2 droite en vue occlusale; G, CBI-2-117, M1 gauche en vue occlusale; $\mathbf{H}, \mathrm{CBI}-2-151$, M3 gauche en vue occlusale; I, CBI-2-139, c1 droite en vue buccale; J, CBI-2-138, c1 gauche en vue buccale; K, CBI-2-136, p4 gauche en vue buccale; L, CBI-2-127, m1 gauche en vue occlusale; M, CBI-2-126, m2 gauche en vue occlusale; N, CBI-2-131, m3 gauche en vue occlusale. Échelle: $1 \mathrm{~mm}$.

La morphologie de Pseudovespertiliavus parva Ravel n. gen., n. sp. présente une forte signature emballonuride mais combine également des caractères que l'on retrouve chez les Nycteridae à des degrés de variations plus ou moins forts:

- la dissymétrie légèrement moins prononcée des ectoflexus (sur M1 uniquement);

- l'aspect plus ouvert du "W» de l'ectolophe de la M1;

- le développement et l'orientation de la postprotocrête entraînant une absence de communication entre la protofosse et le bassin du talon;

- l'aplanissement distal du talon;

- le cingulum lingual d'épaisseur modéré à faible ;

- le développement de l'entoconide plus modéré;

- l'orientation et l'extension de la cristide oblique qui montre une angulation plus accentuée que chez les autres emballonurides et se rapproche de celle observable chez les Nycteridae;

- la faible réduction des structures du talonide de m3 (entoconide et hypoconulide dissociés).

Selon le consensus actuel, les Nycteridae sont groupefrère des Emballonuridae (par ex. : Eick et al. 2005; Simmons 2005a, b; Teeling et al. 2012). Cette hypothèse a été récemment étayée d'un point de vue paléontologique par la présence d'un nyctéridé découvert dans l'Oligocène supérieur du Quercy, qui présente certaines affinités avec les emballonuridés (Sigé 2011b). Pseudovespertiliavus Ravel, n. gen., de par son ancienneté et sa morphologie combinant à la fois des caractères d'Emballonuridae et de Nycteridae, semble proche de la divergence entre les deux familles.
Famille NyCTERIdAe Van Der Hoeven, 1855

Genre Khoufechia Ravel, n. gen.

ESPÈCE TYPE. - Khoufechia gunnelli Ravel, n. sp.

ÉTYMOLOGIE. - Issu de «Khouféche», terme arabe pour désigner une chauve-souris.

Diagnose. - Comme celle de l'espèce type.

Khoufechia gunnelli Ravel, n. sp.

(Figs 18, 19, 20; Tableau 7)

HolotyPe. - Spécimen CBI-2-113, M2 gauche; Figs 18D; 19F.

Matériel eXAminé. - CBI-1-257 (C1 droite; Figs 18A; 19A); CBI-2-123 (Fig. 20A) et CBI-2-124 (C1s gauches; Figs 18B; 19B); CBI-2-121 (Fig. 19D) et CBI-2-122 (P4s droites; Fig. 20B) ; CBI2-120 (P4 gauche; Figs 18C; 19C); CBI-2-114 (Figs 18E; 19G), CBI-2-115 (Fig. 20E) et CBI-2-147 (M1s droites); CBI-2-117 (Figs 18G; 20G), CBI-2-118, CBI-2-142, CBI-2-145 et CBI-1-261 (M1s gauches); CBI-2-150 (M1/2 droite); CBI-2-116 (Figs 18F; 20F), CBI-2-119 (Fig. 20C), CBI-2-140, CBI-2-144, CBI-2-146, CBI-2-141, CBI-2-148, CBI-2-149 et CBI-3-008 (M2s gauches); CBI-2-151 (Fig. 18H) et CBI-2-152 (M3s gauches); CBI-2-139 (c1 droite; Figs 18I; 20G) ; CBI-2-138 (c1 gauche; Figs 18J ; 19J); CBI-1-259 (Fig. 20I), CBI-2-260 (Fig. 19J) et CBI-2-135 (p4s droites; Fig. 20J); CBI-2-134 (Fig. 20K), CBI-2-136 (Figs 18K; 19I) et CBI-2-137 (p4s gauches; Fig. 20H) ; CBI-2-128 (Fig. 20N), CBI-2-129 (Fig. 20L), CBI-2-153, CBI-2-160, CBI-2-167 et CBI3-009 (m1s droites) ; CBI-2-125 (Fig. 19L), CBI-2-127 (Figs 18L; $19 \mathrm{~K})$ et CBI-3-010 (m1s gauches); CBI-2-163 et CBI-2-159 ( $11 / 2 \mathrm{~s}$ droites); CBI-2-156 et CBI-2-168 (m1/2s gauches); CBI-2-130 
TABlEAu 7. - Mesures dentaires (en mm) de Khoufechia gunnelli Ravel n. gen., n. sp. provenant des localités 1, 2 et 3 du Djebel Chambi dans la région de Kasserine, Tunisie. Abréviations: L, longueur; I, largeur; H, hauteur; Ltdr, longueur du trigonide; Itdr, largeur du trigonide; Ltdl, longueur du talonide; Itdl, largeur du talonide. Le signe * derrière une valeur indique un biais dans la mesure causé par l'altération de la couronne.

\begin{tabular}{|c|c|c|c|c|c|c|c|c|c|c|}
\hline Spécimen & Localité & Type de matériel & $\mathbf{L}$ & I & $\mathbf{H}$ & Ltrd & Itrd & LtId & Itld & Remarques \\
\hline CBI-1-257 & Chambi 1 & C1 droite & 1 & 0,64 & 1,56 & - & - & - & - & - \\
\hline CBI-2-123 & Chambi 2 & C1 gauche & 0,97 & 0,54 & 1,44 & - & - & - & - & - \\
\hline CBI-2-124 & Chambi 2 & C1 gauche & 1,05 & 0,66 & 1,33 & - & - & - & - & - \\
\hline CBI-2-121 & Chambi 2 & P4 droite & $0,97^{*}$ & 1,32 & - & - & - & - & - & lobe mésiobuccal cassé \\
\hline CBI-2-122 & Chambi 2 & P4 droite & 0,91 & 1,23 & - & - & - & - & - & - \\
\hline CBI-2-120 & Chambi 2 & P4 gauche & 1,11 & 1,15 & - & - & - & - & - & - \\
\hline CBI-2-114 & Chambi 2 & M1 droite & 1,71 & 1,73 & - & - & - & - & - & - \\
\hline CBI-2-115 & Chambi 2 & M1 droite & $1,55^{\star}$ & $1,54^{\star}$ & - & - & - & - & - & extrêmité distale du talon cassée \\
\hline CBI-2-147 & Chambi 2 & M1 droite & $1,28^{*}$ & $1,64^{*}$ & - & - & - & - & - & talon cassé \\
\hline CBI-2-117 & Chambi 2 & M1 gauche & 1,72 & 1,5 & - & - & - & - & - & - \\
\hline CBI-2-118 & Chambi 2 & M1 gauche & 1,5 & 1,54 & - & - & - & - & - & - \\
\hline CBI-2-142 & Chambi 2 & M1 gauche & $1,11^{*}$ & $1,46^{\star}$ & - & - & - & - & - & $\begin{array}{l}\text { talon manquant ainsi qu'une partie } \\
\text { du flanc lingual du protocône }\end{array}$ \\
\hline CBI-2-145 & Chambi 2 & M1 gauche & 1,28 & 1,38 & - & - & - & - & - & - \\
\hline CBI-1-261 & Chambi 1 & M1 gauche & $1,56^{\star}$ & $1,65^{\star}$ & - & - & - & - & - & talon cassé \\
\hline CBI-2-113 & Chambi 2 & M2 droite & 1,68 & 1,87 & - & - & - & - & - & - \\
\hline CBI-2-116 & Chambi 2 & M2 droite & $1,39^{\star}$ & 1,7 & - & - & - & - & - & styles érodés \\
\hline CBI-2-140 & Chambi 2 & M2 droite & 1,36 & 1,52 & - & - & - & - & - & - \\
\hline CBI-2-146 & Chambi 2 & M2 droite & $1,33^{*}$ & $1,66^{\star}$ & - & - & - & - & - & talon manquant \\
\hline CBI-3-008 & Chambi 3 & M2 droite & 1,23 & 1,72 & - & - & - & - & - & - \\
\hline CBI-2-141 & Chambi 2 & M2 droite & 1,34 & 1,86 & - & - & - & - & - & - \\
\hline CBI-2-148 & Chambi 2 & M2 droite & $1,26^{\star}$ & 1,85 & - & - & - & - & - & région du paracône cassée \\
\hline CBI-2-119 & Chambi 2 & M2 gauche & 1,61 & 1,64 & - & - & - & - & - & métacône manquant \\
\hline CBI-2-143 & Chambi 2 & M2 gauche & $1,43^{*}$ & $1,74^{*}$ & - & - & - & - & - & $\begin{array}{l}\text { couronne très érodée, structures très } \\
\text { émoussées et à peine visibles }\end{array}$ \\
\hline CBI-2-149 & Chambi 2 & M2 gauche & $1,32^{*}$ & $1,64^{*}$ & - & - & - & - & - & talon manquant \\
\hline CBI-2-150 & Chambi 2 & M1/2 droite & $1,12^{*}$ & $1,57^{\star}$ & - & - & - & - & - & couronne très fissurée et érodée \\
\hline CBI-2-151 & Chambi 2 & M3 gauche & 0,93 & 1,38 & - & - & - & - & - & - \\
\hline CBI-2-152 & Chambi 2 & M3 gauche & 0,76 & 1,09 & - & - & - & - & - & - \\
\hline CBI-2-139 & Chambi 2 & c1 droite & $0,73^{*}$ & 0,67 & 1,46 & - & - & - & - & base de la couronne fracturée \\
\hline CBI-2-138 & Chambi 2 & c1 gauche & 0,76 & 0,7 & 1,51 & - & - & - & - & - \\
\hline CBI-1-259 & Chambi 1 & p4 droite & 0,91 & 0,58 & - & - & - & - & - & - \\
\hline CBI-2-135 & Chambi 2 & p4 droite & 0,98 & 0,62 & - & - & - & - & - & - \\
\hline CBI-1-260 & Chambi 1 & p4 droite & 0,95 & 0,73 & - & - & - & - & - & - \\
\hline CBI-2-134 & Chambi 2 & p4 gauche & 0,87 & 0,68 & - & - & - & - & - & - \\
\hline CBI-2-136 & Chambi 1 & p4 gauche & 0,86 & 0,63 & - & - & - & - & - & - \\
\hline CBI-2-137 & Chambi 2 & p4 gauche & 0,98 & 0,66 & - & - & - & - & - & - \\
\hline CBI-2-128 & Chambi 2 & m1 droite & 1,26 & 0,71 & - & 0,75 & 0,66 & 0,49 & 0,71 & - \\
\hline CBI-2-129 & Chambi 2 & m1 droite & 1,29 & 0,69 & - & 0,73 & 0,69 & 0,56 & 0,69 & - \\
\hline CBI-2-153 & Chambi 2 & m1 droite & 1,66 & 0,79 & - & 0,92 & 0,76 & 0,7 & 0,79 & - \\
\hline CBI-2-160 & Chambi 2 & m1 droite & 1,27 & 0,63 & - & 0,75 & 0,63 & 0,48 & 0,62 & - \\
\hline CBI-2-167 & Chambi 2 & m1 droite & 1,45 & 0,72 & - & 0,78 & 0,62 & 0,66 & 0,72 & - \\
\hline CBI-3-009 & Chambi 3 & m1 droite & 1,27 & 0,72 & - & 0,66 & 0,71 & 0,61 & 0,72 & - \\
\hline CBI-2-125 & Chambi 2 & m1 gauche & 1,32 & 0,75 & - & 0,79 & 0,67 & 0,53 & 0,75 & - \\
\hline CBI-2-127 & Chambi 2 & m1 gauche & 1,25 & 0,67 & - & 0,75 & 0,59 & 0,5 & 0,67 & - \\
\hline CBI-2-164 & Chambi 2 & m1 gauche & 1,43 & 0,69 & - & 0,83 & 0,69 & 0,63 & 0,69 & $\begin{array}{l}\text { dent encore enracinée dans un } \\
\text { fragment de dentaire }\end{array}$ \\
\hline CBI-3-010 & Chambi 3 & m1 gauche & 1,11 & 0,53 & - & 0,58 & 0,53 & 0,52 & 0,53 & - \\
\hline CBI-2-130 & Chambi 2 & m2 droite & 1,29 & 0,77 & - & 0,75 & 0,73 & 0,54 & 0,76 & - \\
\hline CBI-2-154 & Chambi 2 & $\mathrm{~m} 2$ droite & 1,28 & 0,84 & - & 0,82 & 0,79 & 0,47 & 0,84 & - \\
\hline CBI-2-158 & Chambi 2 & $\mathrm{~m} 2$ droite & 1,34 & 0,81 & - & 0,77 & 0,76 & 0,56 & 0,81 & - \\
\hline CBI-2-162 & Chambi 2 & m2 droite & 1,11 & 0,67 & - & 0,65 & 0,67 & 0,46 & $0,57^{*}$ & hypoconide cassé \\
\hline CBI-2-165 & Chambi 2 & $\mathrm{~m} 2$ droite & 1,35 & 0,84 & - & 0,7 & 0,78 & 0,63 & 0,84 & - \\
\hline CBI-3-012 & Chambi 3 & m2 droite & 1,63 & 0,66 & - & 0,9 & 0,65 & 0,7 & 0,66 & - \\
\hline CBI-2-126 & Chambi 2 & m2 gauche & 1,28 & 0,75 & - & 0,74 & 0,75 & 0,55 & 0,75 & - \\
\hline CBI-2-155 & Chambi 2 & m2 gauche & 1,25 & 0,63 & - & 0,69 & 0,63 & 0,55 & 0,63 & - \\
\hline CBI-2-157 & Chambi 2 & $\mathrm{~m} 2$ gauche & 1,38 & 0,72 & - & 0,81 & 0,66 & 0,56 & 0,72 & $\begin{array}{l}\text { dent encore enracinée dans un } \\
\text { fragment de dentaire }\end{array}$ \\
\hline CBI-3-007 & Chambi 3 & m2 gauche & 1,2 & 0,65 & - & 0,69 & 0,65 & 0,5 & 0,64 & - \\
\hline CBI-3-011 & Chambi 3 & m2 gauche & 1,47 & 0,89 & - & 0,82 & 0,85 & 0,66 & 0,89 & - \\
\hline CBI-2-159 & Chambi 2 & $\mathrm{~m} 1 / 2$ droite & - & - & - & - & - & 0,65 & 0,65 & talonide uniquement \\
\hline CBI-2-156 & Chambi 2 & $\mathrm{~m} 1 / 2$ gauche & 1,23 & 0,57 & - & 0,64 & 0,54 & 0,6 & 0,57 & - \\
\hline CBI-2-168 & Chambi 2 & $\mathrm{~m} 1 / 2$ gauche & 1,27 & 0,68 & - & 0,68 & 0,61 & 0,58 & 0,68 & - \\
\hline CBI-2-163 & Chambi 2 & m3 droite & 1,1 & 0,71 & - & 0,62 & 0,71 & 0,48 & 0,55 & $\begin{array}{l}\text { dent encore enracinée dans un } \\
\text { fragment de dentaire }\end{array}$ \\
\hline CBI-2-161 & Chambi 2 & m3 droite & 1,14 & 0,71 & - & 0,62 & 0,71 & 0,52 & 0,52 & - \\
\hline CBI-2-166 & Chambi 2 & m3 droite & 1,16 & 0,77 & - & 0,65 & 0,77 & 0,52 & 0,56 & - \\
\hline CBI-2-131 & Chambi 2 & m3 gauche & 1,03 & 0,56 & - & 0,51 & 0,56 & 0,51 & 0,43 & - \\
\hline CBI-2-132 & Chambi 2 & m3 gauche & 1,01 & 0,57 & - & 0,55 & 0,57 & 0,46 & 0,46 & - \\
\hline
\end{tabular}




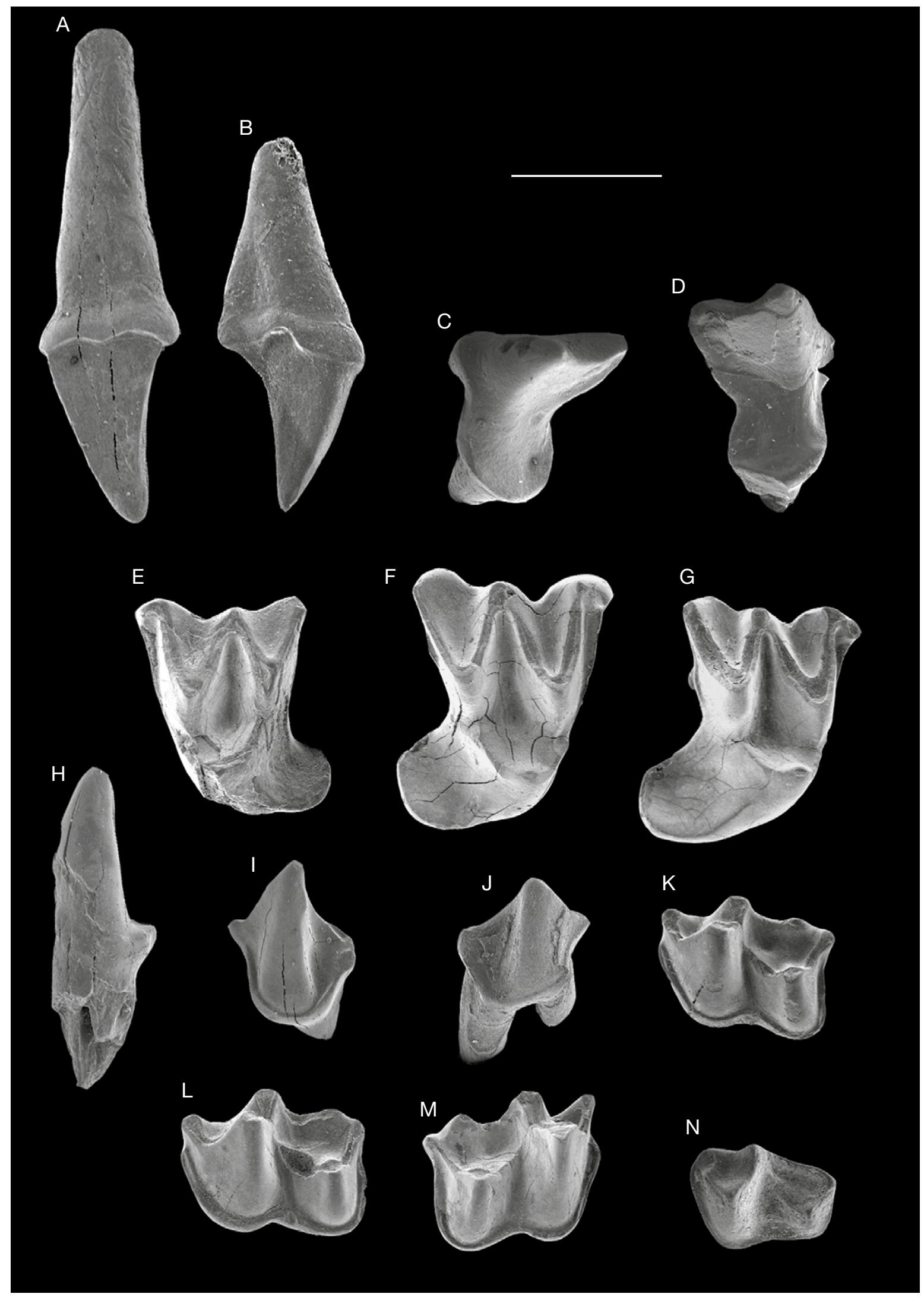

FIG. 19. - Khoufechia gunnelli Ravel n. gen., n. sp. provenant des localités 1 et 2 du Djebel Chambi dans la région de Kasserine, Tunisie: A, CBI-1-257, C1 droite en vue linguale; B, CBI-2-124, C1 gauche en vue linguale; C, CBI-2-120, P4 gauche en vue occlusale; D, CBI-2-121, P4 droite en vue occlusale; E, CBI-2-118, M1 gauche en vue occlusale; F, CBI-2-113, M2 droite en vue occlusale; G, CBI-2-114, M1 droite en vue occlusale; $\mathbf{H}, \mathrm{CBI}-2-138, \mathrm{c} 1$ gauche en vue buccale; I, CBI-2-136, p4 gauche en vue buccale; J, CBI-1-260, p4 droite en vue buccale; K, CBI-2-127, m1 gauche en vue occlusale; L, CBI-2-125, m1 gauche en vue occlusale; M, CBI-2-130, m2 droite en vue occlusale; N, CBI-2-131, m3 gauche en vue occlusale. Échelle: $1 \mathrm{~mm}$. 
(Fig. 19M), CBI-2-154, CBI-2-158, CBI-2-162, CBI-2-165 et CBI-3-012 (m2s droites); CBI-2-126 (Figs 18M; 20M), CBI-2155, CBI-2-157, CBI-3-007 et CBI-3-011 (m2s gauches); CBI2-161 et CBI-2-166 (m3s droites); CBI-2-131 (Figs $18 \mathrm{~N} ; 19 \mathrm{~N}$ ) et CBI-2-132 (m3s gauches; Fig. 20O).

ÉTYMOLOGIE. — Le nom de l'espèce est dédié à Gregg Gunnell pour ses nombreuses contributions à l'étude des chiroptères paléogènes d'Afrique.

LOCALITÉ TYPE ET ÂGE. - Chambi (CBI) loci 1, 2 et 3, Éocène inférieur terminal-Éocène moyen basal, Djebel Chambi, situé dans la région de Kasserine, Tunisie.

Diagnose. - Nycteridae se distinguant des genres Nycteris et Chambinycteris Ravel, n. gen. par les molaires supérieures montrant un bord buccal quasi rectiligne, des styles peu projetés buccalement, un protocône volumineux, une protofosse étendue. P4 à développement transversal, p 4 biradiculée avec un protoconide bien défini, entoconide des m1-2s très réduit mais présent, et terminaison mésiale de la cristide oblique légèrement plus buccale que la jonction entre la postprotocristide et la métacristide.

\section{DESCRIPTION}

La canine supérieure est de petite taille (hauteur inférieure à la largeur des molaires; Figs 18A, B; 19A, B; 20A; Tableau 7). La cuspide principale est incurvée distalement et possède un apex très pointu (plus émoussé et légèrement moins incliné sur CBI-1-257: Figs 18A; 19A). Le tubercule présente une face buccale bombée et une face linguale légèrement creusée. Ces deux surfaces sont délimitées par deux crêtes: une crête de direction mésiolinguale courte et émoussée, et une crête de direction distale plus allongée et élevée. Le cingulum est bien défini sur tout le pourtour de la couronne. Une encoche est visible sur le cingulum, en face linguale. Cette structure est la résultante d'une occlusion avec la cuspide principale de la prémolaire la plus mésiale.

P4 présente une couronne étirée transversalement et fortement cintrée mésiodistalement (Figs 18C; 19C, D; 20B). La couronne de $\mathrm{P} 4$ est dominée par le paracône, situé près du bord buccal et légèrement en avant de la dent. Une crête élevée s'étend depuis l'apex du paracône pour rejoindre le bord distobuccal de la couronne tout en s'incurvant légèrement. La combinaison du paracône et de la postparacrête réalise une large surface tranchante linguale qui s'étend vers le bord lingual du bassin. La partie linguale de la couronne est étiré dans le sens de la largeur formant un talon très bien développé. Le cingulum lingual, très fin, remonte jusqu'à un petit lobe buccomésial.

Les molaires supérieures ont un développement transversal qui est plus accentué chez les M2s (Figs 18D, F; 19F; 20C, F) que chez les M1s (Fig. 18E, G; 19E, G; 20D, E). Le bord buccal n'est pas ou peu incliné mésiolingualement et possède deux encoches (ectoflexus) très profondes de part et d'autre du mésostyle. Ce dernier se projette sur le bord buccal. Le parastyle effectue une flexion mésiale modérée. À l'opposé, le métastyle simplifié prolonge buccalement la postmétacrête. Le mésostyle, bien développé, est très déjeté sur le bord buccal pour se positionner au même niveau que le parastyle et le métastyle. Les crêtes de l'ectolophe sont de longueur équivalente et forment un «W» ouvert et symétrique. La parafosse et la métafosse sont profondes et se situent respectivement au niveau du paracône et du métacône. S'il n'y a pas de différence de taille significative entre le paracône et le métacône, le premier est situé un peu plus lingualement par rapport au second. Les deux tubercules linguaux, très en retrait par rapport au bord buccal, sont très étirés buccolingualement et comprimés mésiodistalement. Le protocône, assez court, est légèrement plus élevé et plus volumineux que le paracône ou le métacône. La préprotocrête remonte buccalement jusqu'à rejoindre le précingulum. Sur certains spécimens (CBI-2113, CBI-2-117, et CBI-2-119), le paralophe, présent mais très court, débute depuis la base linguale du paracône pour terminer distolingualement au niveau d'une triple connexion avec la préprotocrête et le précingulum. La postprotocrête, plus longue que la préprotocrête, remonte distobuccalement jusqu'à la base linguale du métacône, clôturant distalement la protofosse. La protofosse s'étend buccalement jusqu'à la base du mésostyle. Un postcingulum apparaît à la base distale du métacône, mais à l'état de vestige. Le talon est fortement projeté distolingualement (projection plus distale que linguale du talon de M1). Le bassin du talon, associé à la postprotocrête, forment une large et longue surface occlusale concave. Le bassin s'aplanit fortement dans sa partie la plus distolinguale. Le méplat distolingual est ceinturé par un cingulum lingual assez fin.

M3 est particulièrement réduite (CBI-2-151; Fig. 18H), faisant approximativement la moitié de la largeur des M1-2s. Cependant l'ectolophe est constitué du triangle mésial (préparacrête + paracône + postparacrête), d'un mésostyle projeté sur le bord buccal, d'une prémétacrête, et d'un métacône très réduit. Le métacône se positionne légèrement plus buccalement par rapport au paracône. La partie linguale est dominée par un petit protocône réduit. La postprotocrête rejoint la base linguale du métacône tandis que la préprotocrête rejoint soit le précingulum (CBI-2-152), soit directement la base du paracône (CBI-2-151; Fig. 18H). La bordure linguale est arrondie et dépourvue de cingulum.

Certaines canines inférieures peuvent être attribuées à ce taxon de par leur petite taille (Figs 18I, J; 19H; 20G). Leur couronne reste simple, elle est munie d'un seul tubercule peu élevé, droit, et à section ovale. Le cingulum est visible sur tout le contour de la couronne mais devient plus discret (fin) buccalement. Cette structure, fortement inclinée distalement, effectue un méplat distal situé à la base du conide principal.

La p4 est biradiculée. La couronne de cette prémolaire se résume à un seul protoconide incliné distalement, et un talonide d'extension modérée et dépourvu de relief (Figs 18K; 19I, J ; $20 \mathrm{H}-\mathrm{K})$. Une courte crête s'étend depuis le sommet du protoconide, et rejoint un petit lobe mésiolingual situé à la base du conide principal. À l'opposé, une autre crête s'étend vers la bordure linguodistale de la couronne et délimite lingualement le bassin du talonide. Le cingulide encercle de manière homogène la base de la couronne sans jamais s'interrompre.

La couronne des $\mathrm{m} 1-2 \mathrm{~s}$ a une forme très allongée et relativement étroite en vue occlusale (Figs 18L, M; 19K-M; 20L-N). Le trigonide est fortement comprimé buccolingualement. Le paraconide, fortement incliné mésiolingualement, est plus 


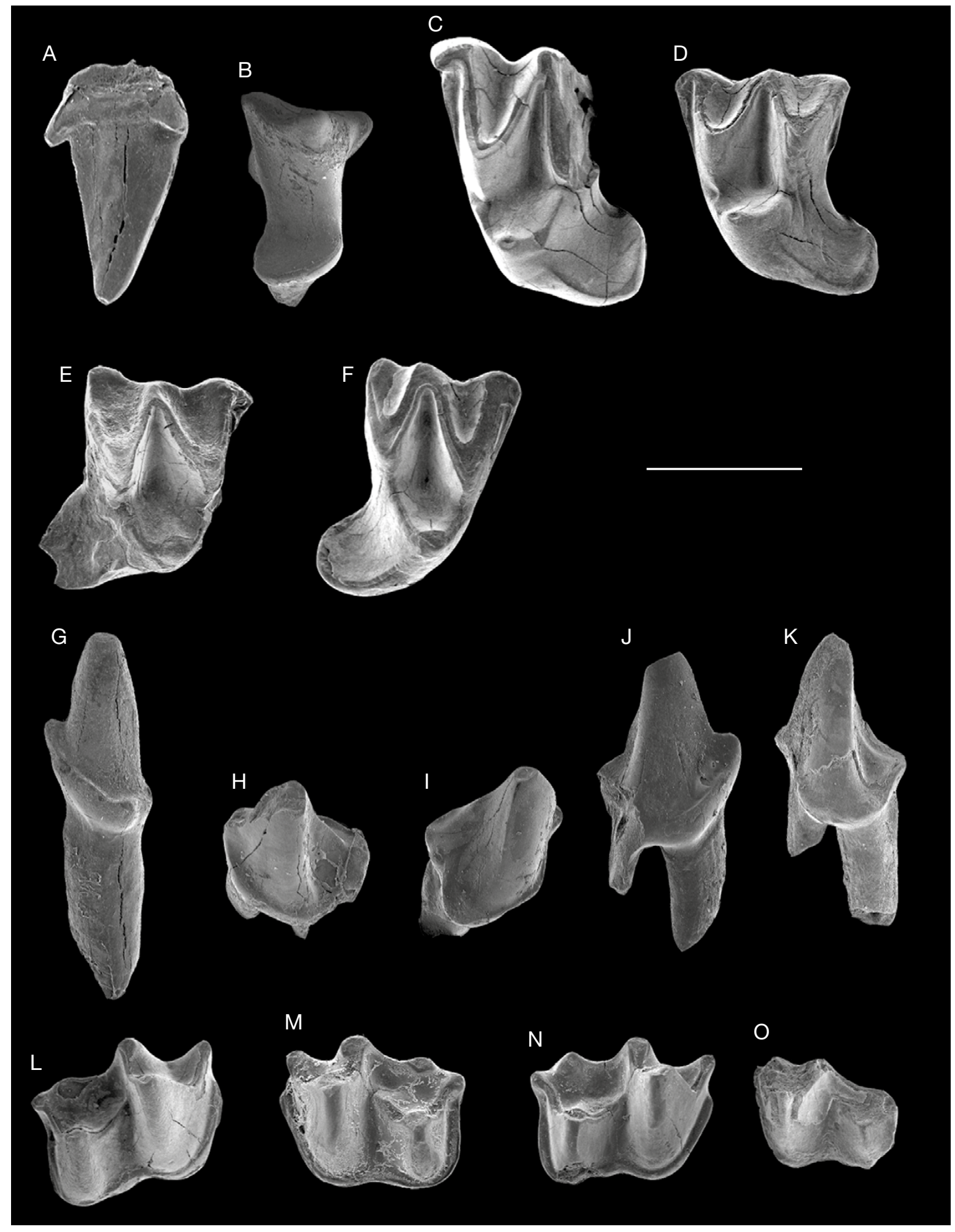

FIG. 20. - Khoufechia gunnelli Ravel n. gen., n. sp. provenant des localités 1 et 2 du Djebel Chambi dans la région de Kasserine, Tunisie: A, CBI-2-123, C1 gauche en vue linguale; B, CBI-2-122, P4 droite en vue occlusale; C, CBI-2-119, M2 gauche en vue occlusale; $\mathbf{D}, \mathrm{CBI}-2-117$, M1 gauche en vue occlusale; E, CBI-2-115, M1 droite en vue occlusale; F, CBI-2-116, M2 droite en vue occlusale; G, CBI-2-139, c1 droite en vue linguale; H, CBI-2-137, p4 gauche en vue semi buccale; I, CBI-1-259, p4 droite en vue semi-buccale; J, CBI-2-135, p4 droite en vue buccale; K, CBI-2-134, p4 gauche en vue buccale; L, CBI-2-129, m1 droite en vue occlusale; M, CBI-2-126, m2 gauche en vue occlusale; N, CBI-2-128, m1 droite en vue occlusale; O, CBI-2-132, m3 gauche en vue occlusale. Échelle: 1 mm.

faible que le métaconide et le protoconide. Le protoconide est très volumineux par rapport aux paraconide et au métaconide. Il apparaît légèrement incliné distalement. Le talonide est un peu plus long mésiodistalement que le trigonide mais approximativement de même largeur buccolinguale. La cristide oblique est rectiligne. Elle relie la base de la muraille distale du 
TABLEAU 8. - Mesures dentaires (en mm) du Vespertilionidae indét. provenant du locus 2 du Djebel Chambi dans la région de Kasserine, Tunisie. Abréviations: L, longueur; I, largeur; Ltdr, longueur du trigonide; Itdr, largeur du trigonide; Ltdl, longueur du talonide; Itdl, largeur du talonide. Le signe * derrière une valeur indique un biais dans la mesure causé par l'altération de la couronne.

\begin{tabular}{|c|c|c|c|c|c|c|c|c|c|}
\hline Spécimen & Localité & $\begin{array}{l}\text { Type de } \\
\text { matériel }\end{array}$ & $\mathbf{L}$ & I & Ltrd & Itrd & Ltld & Itld & Remarques \\
\hline CBI-2-182 & Chambi 2 & $\mathrm{~m} 1 / 2$ droite & 1,63 & $1,04^{\star}$ & 0,74 & 0,93 & 0,89 & $1,04^{*}$ & - \\
\hline CBI-2-179 & Chambi 2 & $\mathrm{~m} 1 / 2$ droite & - & - & - & - & $0,81^{*}$ & 1,03 & talonide uniquement \\
\hline CBI-2-181 & Chambi 2 & $\mathrm{~m} 1 / 2$ droite & - & - & - & - & $0,84^{\star}$ & 1,01 & talonide uniquement \\
\hline CBI-2-183 & Chambi 2 & $\mathrm{~m} 1 / 2$ droite & - & - & - & - & 0,8 & 0,9 & talonide uniquement \\
\hline CBI-2-184 & Chambi 2 & $\mathrm{~m} 1 / 2$ droite & - & - & - & - & 0,76 & 0,84 & talonide uniquement \\
\hline CBI-2-187 & Chambi 2 & $\mathrm{~m} 1 / 2$ droite & - & - & 0,86 & 0,85 & - & - & trigonide uniquement \\
\hline CBI-2-188 & Chambi 2 & $\mathrm{~m} 1 / 2$ droite & - & - & - & - & $0,88^{*}$ & 1,03 & talonide uniquement \\
\hline CBI-2-180 & Chambi 2 & $\mathrm{~m} 1 / 2$ gauche & - & - & - & - & $0,87^{\star}$ & 1,09 & talonide uniquement \\
\hline CBI-2-185 & Chambi 2 & $\mathrm{~m} 1 / 2$ gauche & - & - & - & - & 0,94 & $1,06^{*}$ & talonide uniquement \\
\hline CBI-2-186 & Chambi 2 & $\mathrm{~m} 1 / 2$ gauche & - & - & - & - & $0,89^{\star}$ & 1,05 & talonide uniquement \\
\hline
\end{tabular}
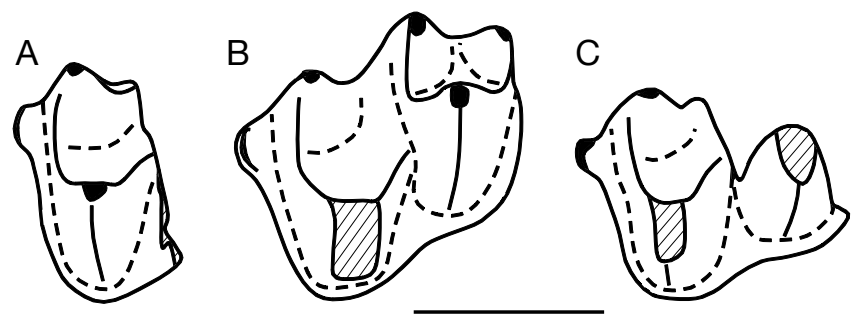

FIG. 21. - Vespertilionidae gen. et sp. indét. provenant du locus 2 du Djebel Chambi dans la région de Kasserine, Tunisie: A, CBI-2-179, talonide de $\mathrm{m} 1 / 2$ droite en vue occlusale; B, CBI-2-182, $\mathrm{m} 1 / 2$ droite en vue occlusale; C, CBI2-181, talonide de $\mathrm{m} 1 / 2$ droite en vue occlusale. Échelle: $1 \mathrm{~mm}$.

trigonide (à un niveau légèrement plus buccal que le métaconide) à la pointe de l'hypoconide. L'hypoconide est la cuspide la plus volumineuse du talonide, et se situe buccolingualement opposé à l'entoconide. La position buccale de l'hypoconide par rapport au protoconide induit un talonide plus large que le trigonide. La postcristide est fortement inclinée distolingualement depuis le sommet de l'hypoconide, et relie l'hypoconide à l'hypoconulide, définissant ainsi une structure nyctalodonte typique. L'entoconide est droit, de taille modérée, et déplacé mésialement par rapport à l'hypoconulide (se rapprochant ainsi du métaconide). Le cingulide est d'épaisseur modérée et continue sur les bordures mésiale, buccale et distale de la couronne.

La m3 est fortement réduite en longueur et largeur par rapport aux m1/2s (Figs $18 \mathrm{~N} ; 19 \mathrm{~N} ; 20 \mathrm{O}$ ). Malheureusement, tous les spécimens présentent une surface occlusale très corrodée qui ne permet pas d'identifier nettement les structures. Toutefois, le trigonide apparaît petit et muni de cuspides très peu développées. Le paraconide paraît moins projeté mésialement, conférant au trigonide la forme d'un triangle équilatéral en vue occlusale. Le talonide est très étroit mais allongé. La postcristide est relativement longue et droite, et rejoint le sommet d'un petit hypoconide situé plus lingualement que le protoconide.

\section{COMPARAISONS ET DISCUSSION}

Le matériel présenté ici rappelle le spécimen CBI-1-016, attribué à un rhinolophoïde par Sigé (1991b). Le spécimen CBI-1-016, incomplet (talon manquant), était rapproché du grand ensemble Rhinolophoïdea par «les proportions et l'angulation des crêtes de l'ectolophe, d'une part, et d'autre part l'exiguïté de la protofossa, la simplicité du protocône et de ses crêtes, la présence nettement indiquée d'un lobe distal lingual». L'auteur soulignait également l'originalité des orientations et connections des protocrêtes qui diffèrent de la plupart des rhinolophoïdes fossiles et récents. L'abondance du matériel révélé ici permet de mieux appréhender la morphologie dentaire de ce taxon et d'affiner son statut systématique. Ainsi, Khoufechia gunnelli Ravel n. gen., n. sp. présente un ensemble de caractères que l'on retrouve de manière généralisée chez les Nycteridae (famille monogénérique [Nycteris Geoffroy \& Cuvier, 1795]) :

- la faible hauteur des canines supérieures;

- le développement de l'ectolophe formant un «W» ouvert;

- la projection des styles sur la bordure buccale;

- l'absence d'inclinaison de la bordure buccale;

- la présence d'un double ectoflexus;

- le postcingulum vestigial;

- la relative réduction du complexe protocône + protocrêtes + protofosse;

- l'orientation de la postprotocrête qui rejoint la base du métacône;

- le fort développement du talon dont la projection est à tendance distolinguale;

- le cingulum lingual d'épaisseur constante et modérée;

- la forte réduction de M3;

- l'ouverture importante des trigonides des m1-2s, induite par la projection mésiale du paraconide;

- l'entoconide réduit (parfois complètement absent chez les Nycteridae actuels) et éloigné de l'hypoconulide;

- l'inclinaison forte de la crête oblique.

À côté de ces ressemblances existe une liste de différences plus ou moins prononcées marquant l'originalité du taxon tunisien par rapport au genre Nycteris:

- le cingulum de la canine est beaucoup moins épais chez Khoufechia Ravel, n. gen. que chez Nycteris. De plus, chez ce dernier le cingulum est fortement courbé face linguale;

- la P4 de Khoufechia Ravel, n. gen. possède un développement transversal plus prononcé que les P4s de Nycteris. Le talon est plus étendu lingualement; 


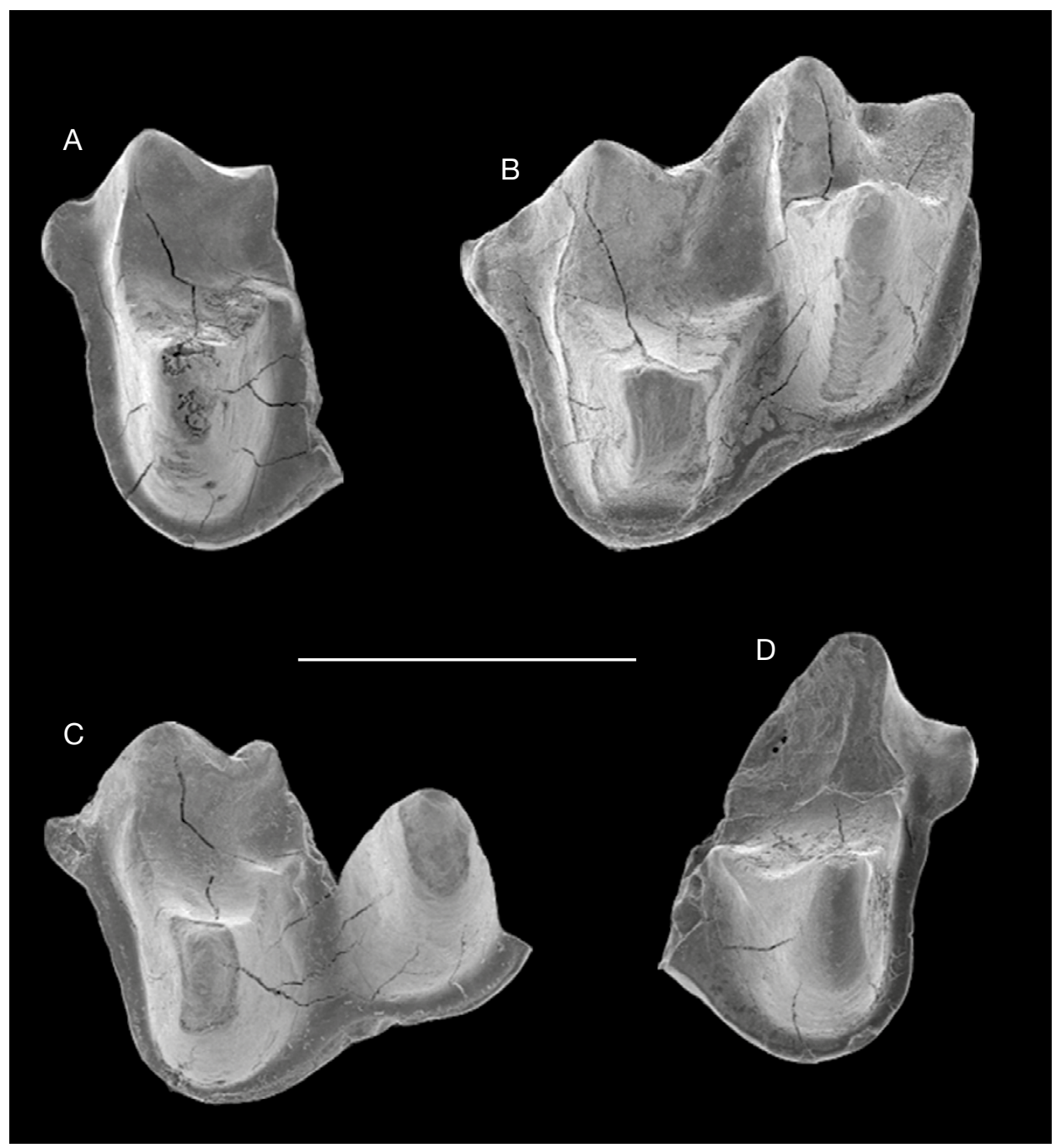

FIG. 22. - Vespertilionidae gen. et sp. indét. provenant du locus 2 du Djebel Chambi dans la région de Kasserine, Tunisie: A.CBI-2-179, talonide de m1/2 droite en vue occlusale; B, CBI-2-182, m1-2 droite en vue occlusale; C, CBI-2-181, talonide de m1/2 droite en vue occlusale; $\mathbf{D}$, CBI-2-180, talonide de m1/2 gauche en vue occlusale. Échelle: $1 \mathrm{~mm}$.

- les ectoflexus des molaires supérieures sont peu prononcés chez Khoufechia Ravel, n. gen. Ceci s'accompagne de styles associés à l'ectolophe modérément déjetés buccalement en comparaison des formes récentes qui possèdent de profonds ectoflexus avec des styles très déjetés buccalement;

- les M1s et M2s de Khoufechia Ravel, n. gen. présentent un protocône plus volumineux, des crêtes associées plus longues (en particulier la postprotocrête qui est absente chez Nycteris) et une protofosse légèrement plus étendue;

- certains spécimens attribués à Khoufechia Ravel, n. gen. présentent un paralophe. Cette structure n'apparait jamais sur les molaires supérieures de Nycteridae;

- le cingulum lingual encerclant le bassin du talon est moins épais chez Khoufechia Ravel, n. gen. que chez Nycteris;

- la p4 de Khoufechia Ravel, n. gen. est plus développée, biradiculée, et présente un protoconide et un talonide bien développés. Cette prémolaire chez les Nycteridae est extrêmement réduite et compressée mésiodistalement;
- l'entoconide observable sur les m1/2s de Khoufechia Ravel, n. gen. est plus développé et moins déplacé mésialement. Chez les Nycteridae, l'entoconide est à peine visible, parfois absent, et se situe à la base distale du métaconide.

Le registre fossile des Nycteridae est particulièrement pauvre et il comprend également un matériel dont l'attribution systématique est incertaine. Le plus ancien Nycteridae référencé à ce jour est Chibanycteris herberti Sigé, Thomas, Sen, Gheerbrant, Roger \& Al-Sulaimani, 1994 de l'Oligocène inférieur du Sultanat d'Oman (Taqah, Dhofar; Sigé et al. 1994). Les M2s de Khoufechia Ravel, n. gen. different de la M2 de Chibanycteris Sigé, Thomas, Sen, Gheerbrant, Roger \& Al-Sulaimani, 1994 par: - la présence de deux ectoflexus plus profonds;

- le développement plus important du protocône et de ses crêtes ainsi qu'une protofosse plus étendue;

- le talon projeté plus distalement et formé par un bassin nettement plus étendu;

- le cingulum lingual plus fin;

- l'absence d'entoconide sur m3. 
TABLEAu 9. - Mesures dentaires (en mm) Drakonycteris glibzegdouensis Ravel n. gen., n. sp. provenant du niveau HGL50 du Glib Zegdou situé dans les Gour Lazib, Algérie. Abréviations: L, longueur; I, largeur; Ltdr, longueur du trigonide; Itdr, largeur du trigonide; Ltdl, longueur du talonide; Itdl, largeur du talonide. Le signe * derrière une valeur indique un biais dans la mesure causé par l'altération de la couronne.

\begin{tabular}{|c|c|c|c|c|c|c|c|c|}
\hline Spécimen & Localité & Type de matériel & $\mathbf{L}$ & I & Ltrd & Itrd & Ltld & Itld \\
\hline UM/HGL50-416 & HGL50, Glib Zegdou & P4? droite & 1,45 & 1,41 & - & - & - & - \\
\hline UM/HGL50-415 & HGL50, Glib Zegdou & M1 droite & 2,15 & 2,78 & - & - & - & - \\
\hline UM/HGL50-414 & HGL50, Glib Zegdou & M2 droite & 2,15 & 3,02 & - & - & - & - \\
\hline UM/HGL50-417 & HGL50, Glib Zegdou & m1-2 gauche & $1,83^{\star}$ & 1,29 & $0,90^{*}$ & 1,29 & $0,93^{*}$ & 1,29 \\
\hline UM/HGL50-418 & HGL50, Glib Zegdou & m3 gauche & 1,26 & 0,89 & 0,68 & 0,89 & 0,58 & 0,63 \\
\hline UM/HGL50-419 & HGL50, Glib Zegdou & m3 gauche & $1,29^{\star}$ & 0,87 & 0,7 & 0,87 & $0,59^{\star}$ & 0,87 \\
\hline
\end{tabular}

En plus de ces différences, les m3s de Khoufechia Ravel, n. gen. n'ont pas d'entoconide, ce qui l'éloigne de Chibanycteris qui possède les trois cuspides du talonide de $\mathrm{m} 3$ bien individualisées.

La pauvreté du matériel provenant de la péninsule arabique ne permet pas une comparaison plus poussée. Mais les nettes différences présentées ici écartent la possibilité que le taxon de Chambi appartienne au genre Chibanycteris.

Sigé (2011b) mentionne la présence du genre Nycteris dans des localités karstiques du Quercy daté de l'Oligocène supérieur (Mas de Pauffié et La Devèse, MP26). Le taxon du Quercy présente des similitudes avec les Emballonuridae, des affinités qui ont conduit Remy et al. (1987) à le voir comme un Emballonuridae indét. Cette attribution taxinomique est basée sur : la cuspide de la canine supérieure acérée, la morphologie de P4, le développement distolingual du talon, et l'ectoflexus antérieur au mésostyle (peu courant chez les Nycteridae). Ces similitudes ont conduit Sigé (2011b) à appuyer la thèse de la parenté entre les Nycteridae et les Emballonuridae. Khoufechia gunnelli Ravel n. gen., n. sp. présente également un talon bien développé et une canine supérieure acérée. De plus, chez Khoufechia Ravel, n. gen., il est possible d'observer certains caractères, autres que ceux cités précédemment, qui se retrouvent de manière généralisée chez les Emballonuridae: le développement du protocône, de la postprotocrête et de la protofosse, la plus faible projection des styles de l'ectolophe, la p4 non réduite et l'entoconide mieux individualisé. Ces caractères vont dans le sens également d'une relation phylogénétique étroite entre les Nycteridae et les Emballonuridae, relation soutenue initialement par Miller (1907).

Super-famille VESPERTILIONOIDEA Gray, 1821 Famille VeSPERTILIONIDAE Gray, 1821

gen. et sp. indét.

(Figs 21, 22; Tableau 8)

MATÉRIEL EXAMINÉ. - CBI-2-182 (m1/2 droite; Figs 21B; 22B); CBI-2-187 (trigonide de $\mathrm{m} 1 / 2$ droite) ; CBI-2-179 (Figs 21A; 22A), CBI-2-181 (Figs 21C; 22C), CBI-2-183, CBI-2-184 et CBI-2-188 (talonides de $\mathrm{m} 1 / 2$ droites); CBI-2-180 (Fig. 22D), CBI-2-185 et CBI-2-186 (talonides de $\mathrm{m} 1 / 2$ gauches).

LOCALITÉ ET ÂGE. - Chambi (CBI) locus 2, Éocène inférieur terminal-Éocène moyen basal, Djebel Chambi, situé dans la région de Kasserine, Tunisie.

\section{DESCRIPTION}

Ce taxon est uniquement représenté par des spécimens fragmentaires attribuables à des molaires inférieures de taille modérée (en comparaison des autres espèces). L'essentiel du matériel consiste en des fragments de talonides et deux molaires inférieures quasi-complètes. Le trigonide est plutôt comprimé en largeur et longueur, beaucoup plus court et étroit que le talonide. Ce dernier constitue la majeure partie de la dent. Les cuspides du trigonide sont peu élevées mais volumineuses. Le paraconide, plus petit que le métaconide et le protoconide, se projette linguomésialement. Le métaconide est droit, et presque équivalent en taille et volume au protoconide. La crête oblique est rectiligne et relie la base de la face distale du trigonide à l'hypoconide en s'inclinant buccodistalement. L'hypoconide est massif et occupe une position beaucoup plus buccale que le protoconide, rendant ainsi le bassin du talonide très large buccolingualement. La postcristide se projette lingualement et rejoint directement l'entoconide formant une haute paroi distale. L'hypoconulide est isolé et se situe à la base distolinguale de l'entoconide tout en étant très déjeté vers l'extrémité distale de la dent. Le talonide dessine ainsi une structure nettement myotodonte qui est par ailleurs caratéristique de la plupart des Vespertilionidae. L'entoconide, situé légèrement distalement par rapport à l'hypoconide, prend la forme d'une cuspide droite possédant une base très large et robuste. L'entocristide fait la jonction entre le sommet de l'entoconide et l'apex du métaconide, érigeant ainsi une haute muraille linguale au bassin du talonide. Le cingulide débute au niveau de la base mésiobuccale du métaconide et rejoint sans interruption l'hypoconulide en position distolinguale.

\section{Comparaison et discussion}

L'absence de tout autre type de dent pouvant être associé limite les comparaisons pour ce taxon. Ces molaires inférieures sont caractérisées par une structure clairement myotodonte: c'est-à-dire que la postcristide se connecte directement entre l'hypoconide et l'entoconide, isolant ainsi l'hypoconulide. Ce type de structure se retrouve très fréquemment chez les Philisidae, Vespertilionidae et les Noctilionidae (Menu \& Sigé 1971; Menu 1985, 1987). La présence d'un entoconide haut et fort, un hypoconulide bas, un talonide plus large que le trigonide, sont des caractères rapprochant ces spécimens de Chambi de l'ensemble Vespertilionidae. Khonsunycteris 

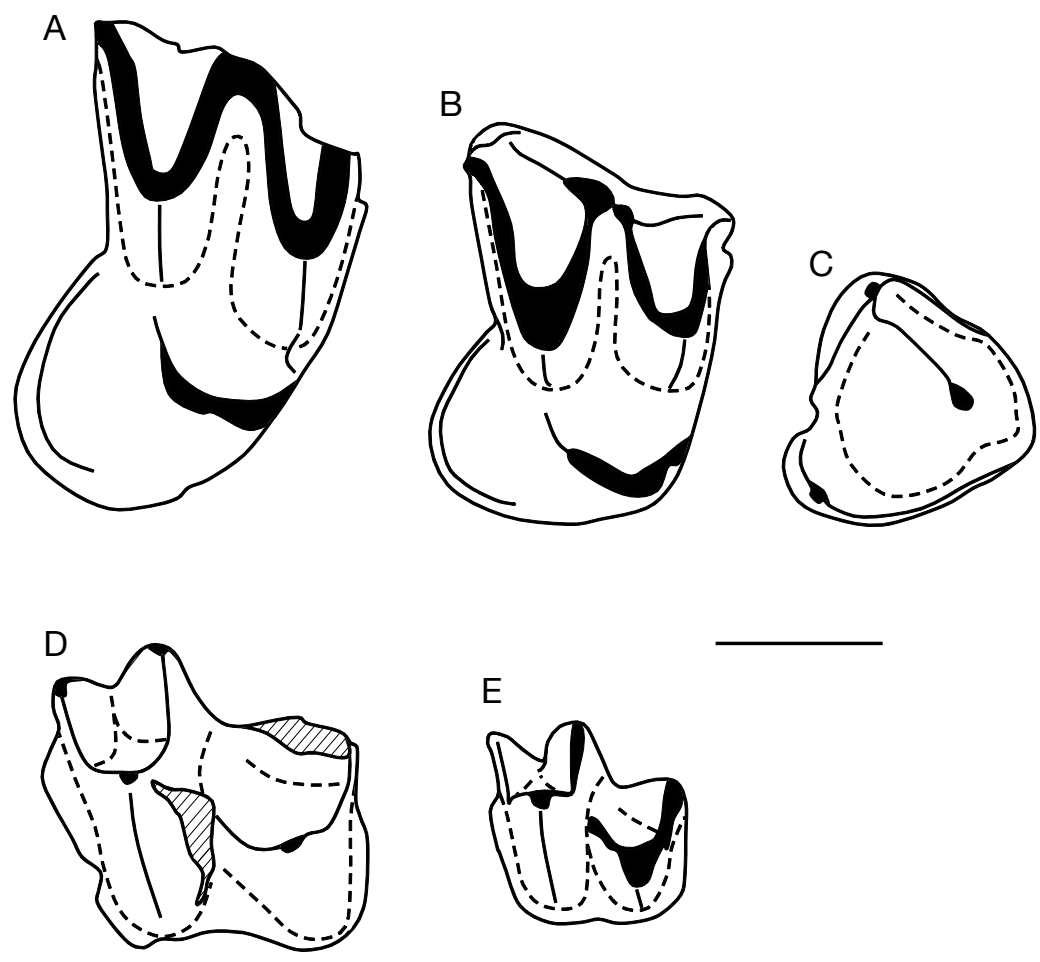

FIG. 23. - Drakonycteris glibzegdouensis Ravel n. gen., n. sp. provenant du niveau HGL50 du Glib Zegdou situé dans la région des Gour Lazib en Algérie: A, UM/HGL50-414, M2 droite en vue occlusale; B, UM/HGL50-415, M1 droite en vue occlusale; C, UM/HGL50-416, P3 droite en occlusale; D, UM/HGL50-417, $\mathrm{m} 1 / 2$ gauche en vue occlusale; E, UM/HGL50-418, m3 gauche en vue occlusale. Échelle: $1 \mathrm{~mm}$.

aegypticus Gunnell, Simmons\& Seiffert, 2008 est un Vespertilionidae fossile attesté dans le niveau L-41 (Éocène supérieur terminal) du Fayum en Égypte (Gunnell et al. 2008). Khonsunycteris aegypticus présente également une myotodontie et une morphologie très semblable au Vespertilionidae de Tunisie. Seules quelques différences sont notables entre les deux taxons: l'entocristide présente une courbure très prononcée sur les spécimens de Chambi alors qu'elle est rectiligne chez $K$. aegypticus; le bord buccal des molaires de Chambi est très incliné distalement (accompagné d'un hypoconide nettement plus bas que le protoconide), cette inclinaison paraît moins prononcée sur les molaires de $K$. aegypticus. Un matériel plus abondant reste toutefois nécessaire afin de préciser le degré d'affiliation entre ces deux taxons fossiles.

Famille indét.

Genre Drakonycteris Ravel, n. gen.

ESPÈCE TYPE. - Drakonycteris glibzegdouensis Ravel, n. sp.

ÉTYMOLOGIE. - Le nom de genre est la combinaison des mots grecs

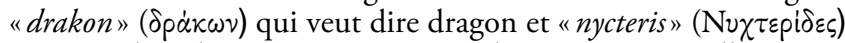
qui veut dire chauve-souris. Le nom du genre est une allusion au contexte général que dégage la découverte de ce chiroptère fossile sur le flanc de la butte du Glib Zegdou. De plus, il souligne la morphologie robuste mais originale du matériel dentaire qui lui est attribué.

Diagnose. - Comme celle de l'espèce type.
Drakonycteris glibzegdouensis Ravel, n. sp. (Figs 23, 24; Tableau 9)

HoLOTYPE. - Spécimen UM/HGL50-415, M1 droite (Figs 23B; 24B).

MatéRIel eXAminé. - UM/HGL50-416 (P3 droite; Figs 23C; 24C); UM/HGL50-414 (M2 droite; Fig. 23A; 24A); UM/HGL50417 (m1/2 gauche; Figs 23D; 24D); UM/HGL50-418 (Figs 23E; 24E) et UM/HGL50-419 (m3s gauches; Fig. 24F).

ÉTYMOLOGIE. - Le nom de l'espèce fait référence à la bute témoin du Glib zegdou, située dans les Gour Lazib, Algérie.

LOCALITÉ TYPE ET ÂGE. - Niveau HGL50 du Glib Zegdou, Éocène inférieur terminal-Éocène moyen basal, situé dans la région des Gour Lazib (Sahara, Hammada du Dra) en Algérie.

DiAgNose. - Chiroptère possédant une morphologie dentaire robuste avec cuspides puissants, crêtes acérées; une P3 massive et triangulaire munie d'un paracône très fort mais dépourvu de bassin; ectolophe de la M1 et de la M2 avec crêtes latérales subparallèles et à fort développement transversal, présence de deux mésostyles jumelés et accolés sur M1, cingulum buccal épais et continu, parastyle très réduit, talon développé dans le sens distolingual et ceinturé par un cingulum lingual discontinu ; molaires inférieures pourvues d'un trigonide très étroit et d'un talonide très allongé.

\section{DESCRIPTION}

Le matériel qui est attribué à cette espèce est très pauvre mais présente des dimensions significativement plus grandes que celles des spécimens attribués à Pseudovespertiliavus parva Ravel n. gen., n. sp. (voir plus haut, Tableaux 6, 9). 
La $\mathrm{P} 3$ possède trois racines dont deux en position buccale et une en position linguale (Figs $23 \mathrm{C} ; 24 \mathrm{C}$ ). La couronne prend une forme triangulaire en vue occlusale, légèrement plus large que longue. La bordure linguodistale est légèrement creusée formant un lobe lingual partiellement individualisé. Le paracône est particulièrement massif et haut, constituant l'essentiel de la couronne. Son flanc lingual est pincé dans le sens mésiodistal. La face buccale est beaucoup moins étendue et moins bombée. La préparacrête est très courte et se connecte au cingulum à l'extrémité mésiale de la couronne. La postparacrête s'étend depuis l'apex du paracône vers la bordure distobuccale de la dent, au niveau d'un relief cuspidé initié par le cingulum. Une petite cuspide, située sur le cingulum, apparaît dans la région distolinguale. Le cingulum, d'épaisseur variable, est continu sur tout le contour de la couronne.

Les molaires supérieures ont un développement à dominante transversale, plus prononcé sur M2 (Figs 23A; 24A). Le bord buccal de M1 est très incliné mésiolingualement et possède un ectoflexus à peine décelable situé mésialement par rapport au mésostyle (Figs 23B ; 24B). La bordure buccale est cernée par un épais cingulum qui effectue la jonction entre le parastyle et le métastyle. Le parastyle est très réduit et se résume à une petite inflexion buccomésiale de la préparacrête. Une profonde encoche est visible en dessous du parastyle permettant l'imbrication de l'extrémité distale de la postparacrête de P4 (cette dent étant inconnue). Le mésostyle est dédoublé sur M1. Les deux mésostyles sont jumelés et accolés, si bien qu'il n'y a pas d'encoche les séparant. Le mésostyle distal est le plus volumineux des deux. Il prolonge buccodistalement la prémétacrête. La M2 ne semble pas caractérisée par ce dédoublement du mésostyle; on retrouve à la place une large surface d'usure arrondie et continue. Le métastyle est semblable au parastyle en effectuant une petite flexion distobuccale tout en prolongeant la postmétacrête. Les crêtes de l'ectolophe sont subparallèles et étirées dans le sens de la largeur, conférant à l'ectolophe une forme en "W" peu ouverte. Le paracône et le métacône ont un volume équivalent. Ces deux cuspides, sur M1, se positionnent sur le même axe mésiobuccal alors que sur M2, le paracône est décalé lingualement par rapport au métacône. Le paralophe est visible à la base du paracône de UM/HGL50-414 (Figs 23A; 24A). Cette petite structure semble rejoindre la préprotocrête, mais une cassure à cet endroit ne permet pas de confirmer cette jonction. Le protocône domine en hauteur et en volume le paracône et le métacône. Il est déjeté vers la partie mésiale de la molaire mais conserve une position plus distale par rapport au paracône. Les deux molaires présentent une courte préprotocrête qui rejoint le précingulum étroit. La postprotocrête est plus longue et présente un tranchant qui s'accentue vers son extrémité distobuccale. Cette crête, très incurvée lingualement s'oriente vers la base linguale du métacône sans pour autant l'atteindre. Les molaires sont pourvues d'un talon arrondi et projeté distolingualement. Le cingulum lingual, d'épaisseur modérée, encercle la partie distolinguale du talon et remonte vers l'extrémité linguale du postcingulum. Sur M1, ces deux structures sont connectées pour ne former plus qu'un seul cingulum effectuant toute la bordure distale de la dent. Une portion du cingulum lingual, discontinue avec la partie postérieure, est visible à la base mésiolinguale du protocône.

Une seule $\mathrm{m} 1 / 2$ appartenant à cette espèce a été identifiée dans le matériel récolté au Glib Zegdou (UM/HGL50417 ; Figs 23D ; 24D). Cette molaire inférieure possède un trigonide très étroit et un talonide dont la longueur dépasse celle du trigonide. Le paraconide est la plus faible des trois cuspides constituant le trigonide. Le métaconide est plus robuste et droit par rapport au paraconide. Le protoconide, le plus volumineux des trois, présente un flanc fortement pincé mésiodistalement. Le talonide est altéré et sa structure ne peut pas être décrite avec précision. La partie distale de la molaire est plus basse que le trigonide mais leurs largeurs sont identiques. L'hypoconide est pincé mésiodistalement (pincement comparable à celui du protoconide). La cristide oblique, assez longue, semble rejoindre la base distale du trigonide au niveau de l'encoche entre le protoconide et le métaconide. Le cingulide bien qu'incomplet à cause de l'altération semble continu, encerclant toute la couronne à l'exception de la bordure linguale.

La troisième molaire inférieure est beaucoup plus réduite en taille que la m1/2 (environ deux fois plus petite; Figs 23E; $24 \mathrm{E}, \mathrm{F}$; Tableau 8). Le trigonide est bien formé, présentant une morphologie proche de celle observée sur $\mathrm{m} 1 / 2$, avec cependant un paraconide plus effilé. La différence de longueur entre le trigonide et le talonide est beaucoup plus ténue sur m3. Le talonide est dépourvu d'hypoconulide. La postcristide fait la jonction directe entre l'entoconide très réduit et l'hypoconide. Le cingulide de ce type de dent montre une épaisseur homogène sur les bordures mésiale, buccale et distale de la couronne.

\section{Comparaison eT discussion}

Le développement transversal des molaires supérieures de Drakonycteris glibzegdouensis Ravel n. gen., n. sp. rappelle les formes primitives que l'on retrouve dans l'Éocène inférieur du Bassin de Paris, soit Icaronycteris menui et Archaeonycteris brailloni (Russell et al. 1973). De plus, la P3 de D. glibzegdouensis Ravel n. gen., n. sp. est massive, triradiculée, de forme triangulaire avec un lobe lingual de faible extension. Cependant, ce nouveau taxon du Glib Zegdou présente un ensemble de caractères qui l'écarte incontestablement des chiroptères basaux (comprenant Onychonycteridae, Icaronycteridae, Archaeonycteridae, Hassianycteridae et Palaeochiropterygidae):

- l'aspect massif des couronnes dentaires;

- le bord buccal de M1 fortement incliné mésiodistalement; - la présence d'un très faible ectoflexus mésial au mésostyle (centrale et très profond chez les formes primitives, parfois double chez les Palaeochiropterygidae);

- le développement transversal de l'ectolophe et le parallélisme des crêtes;

- le mésostyle bien développé et projeté sur la bordure buccale; - le double mésostyle sur M1; 


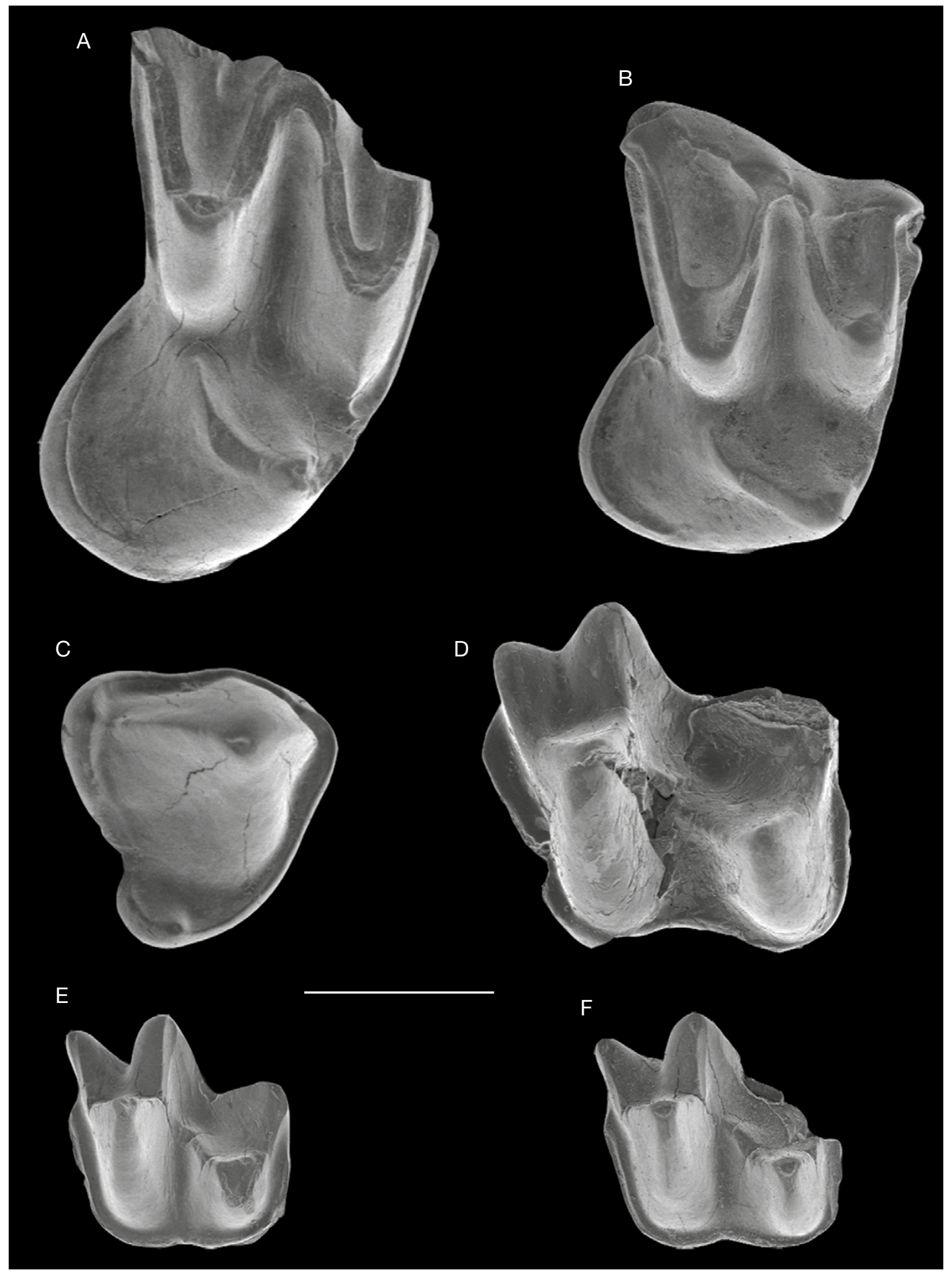

FIG. 24. - Drakonycteris glibzegdouensis Ravel n. gen., n. sp. provenant du niveau HGL50 du Glib Zegdou situé dans la région des Gour Lazib en Algérie: A, UM/ HGL50-414, M2 droite en vue occlusale; B, UM/HGL50-415, M1 droite en vue occlusale; C, UM/HGL50-416, P3 droite en vue occlusale; D, UM/HGL50-417, m1/2 gauche en vue occlusale; $\mathbf{E}$, UM/HGL50-418, m3 gauche en vue occlusale; F, UM/HGL50-419, m3 gauche en vue occlusale. Échelle: 1 mm.

- la forte réduction du parastyle;

- la profonde encoche buccomésiale sous le parastyle;

- la projection du talon;
- le cingulum lingual discontinu d'épaisseur modérée; - le trigonide très court mais large;

- l'élongation du talonide par rapport au trigonide. 
TABLEAU 10. - Mesures dentaires (en mm) Chambinycteris pusilli Ravel n. gen., n. sp. provenant des loci 2 et 3 du Djebel Chambi dans la région de Kasserine, Tunisie. Abréviations: L, longueur; I, largeur; H, Hauteur. Le signe * derrière une valeur indique un biais dans la mesure causé par l'altération de la couronne.

\begin{tabular}{lllllll}
\hline Spécimen & Localité & Type de matériel & $\mathbf{L}$ & $\mathbf{I}$ & $\mathbf{H}$ & Remarques \\
\hline CBI-2-196 & Chambi 2 & C1 gauche & 1,07 & 0,72 & 1,44 & - \\
CBI-2-197 & Chambi 2 & C1 gauche & 0,99 & 0,73 & 1,46 & - \\
CBI-2-204 & Chambi 2 & M1 gauche & 1,5 & 1,52 & - & - \\
CBI-3-016 & Chambi 3 & M1 gauche & $1,36^{\star}$ & 1,43 & - & région du paracône cassée \\
CBI-2-206 & Chambi 2 & c1droite & $0,85^{\star}$ & 0,68 & 1,1 & tubercule cassé à mi-hauteur \\
CBI-2-207 & Chambi 2 & c1 droite & 0,85 & 0,77 & 0,87 & - \\
\hline
\end{tabular}

La projection du talon des M1 et M2 est un caractère généralisé au sein des formes rhinolophoïdes, emballonuroïdes et de certaines formes noctilionoïdes. La morphologie des molaires supérieures de Drakonycteris glibzegdouensis Ravel n. gen., n. sp. s'écarte significativement du grand ensemble des Rhinolophoidea par: - le développement de la P3;

- la forte inclinaison du bord buccal induit par le parastyle très lingual par rapport au métastyle;

- l'ectoflexus unique et peu profond;

- le resserrement mésiodistal du «W» de l'ectolophe;

- le double mésostyle présent sur M1;

- la forte réduction du parastyle;

- le protocône puissant;

- la compression mésiodistale du trigonide.

La forme du Glib Zegdou possède des caractères partagés avec les Emballonuridae qui sont également représentés dans la même localité avec Pseudovespertiliavus parva Ravel n. gen., n. sp. (voir plus haut):

- la présence d'une P3 (la P3 est présente et bien développée chez les formes fossiles telles que Vespertiliavus et Tachypteron Storch, Sigé \& Habersetzer, 2002, elle est cependant réduite ou absente chez les formes actuelles);

- l'inclinaison de la bordure buccale de M1;

- l'ectoflexus mésial;

- le développement transversal de l'ectolophe;

- l'ouverture distale de la protofosse;

- la compression du trigonide;

- la réduction des $\mathrm{m} 3 \mathrm{~s}$.

Mais ces similitudes s'accompagnent de nombreuses différences qui ne permettent pas d'associer le taxon algérien avec l'ensemble emballonuroïde, notamment:

- l'élongation transversale des molaires supérieures;

- la forte réduction du parastyle;

- le double mésostyle sur M1;

- le parallélisme des crêtes de l'ectolophe;

- la simplicité du talon dépourvu de bassin et incliné lingualement;

- le cingulum lingual discontinu;

- la largeur équivalente du trigonide et du talonide.

Le développement de P3, le double mésostyle, et la forte extension du talonide se retrouvent chez certains Noctilionoïdea insectivores. Cet ensemble, principalement sud-américain, présente de manière générale des caractères qui ne sont pas observés chez Drakonycteris Ravel, n. gen. Il s'agit notamment:

- du talon individualisé formant un lobe distolingual;

- de l'hypocône bien développé;

- de l'ectolophe ouvert;
- du double ectoflexus très prononcé et accentué par une forte projection buccale du mésostyle;

- du parastyle en crochet projeté mésialement;

- de la compression buccolinguale du protocône.

La projection linguodistale du talon ne paraît pas compatible avec une morphologie vespertilionoïde. Toutefois, Drakonycteris Ravel, n. gen. possède certaines similitudes avec cet ensemble de chiroptères:

- le développement transversal des molaires supérieures;

- l'orientation et l'étirement buccomésial des crêtes de l'ectolophe; - la simplicité de la partie linguale des molaires supérieures;

- la puissance du protocône;

- l'élongation du talonide.

La présence de deux mésostyles sur la M1 de Drakonycteris Ravel, n. gen. est une caractéristique des Philisidae (Sigé 1985, 1991b; Sigé et al. 1994; Gunnell et al. 2008; Ravel et al. 2012, 2015). De plus, le taxon algérien partage avec les Philisidae une denture robuste dénotant une certaine puissance de l'appareil masticateur. Cependant, Drakonycteris Ravel, n. gen. differe des Philisidae par le rapprochement et la réduction des mésostyles (séparés par une profonde encoche chez Philisis Sigé, 1985 et Witwatia Gunnell, Simmons \& Seiffert, 2008), l'inclinaison du bord buccal de M1, l'absence d'une extension distobuccale de la prémétacrête, la forte réduction du parastyle, la profonde encoche mésiobuccale et la projection distolinguale du talon.

Drakonycteris Ravel, n. gen. présente une morphologie dentaire, particulièrement au niveau des molaires supérieures, combinant un ensemble de caractères inédits au sein des chiroptères ne permettant pas de statuer sur son rang familial. Il est possible que ce taxon reflète, à l'image des Philisidae, l'endémisme de certains chiroptères africains au cours du Paléogène. Cependant en l'absence de matériel plus complet l'attribution familiale reste en suspens. De plus, il est tout à fait possible que de nouvelles découvertes fossiles puissent confirmer la présence d'une nouvelle famille de chiroptère au Glib Zegdou.

Famille indét.

Genre Chambinycteris Ravel, n. gen.

ESPÈCE TYPE. - Chambinycteris pusilli Ravel, n. sp.

ÉTYMOLOGIE. - Le nom de genre fait référence à la localité de Chambi où les spécimens ont été récoltés.

Diagnose. - Comme celle de l'espèce type. 


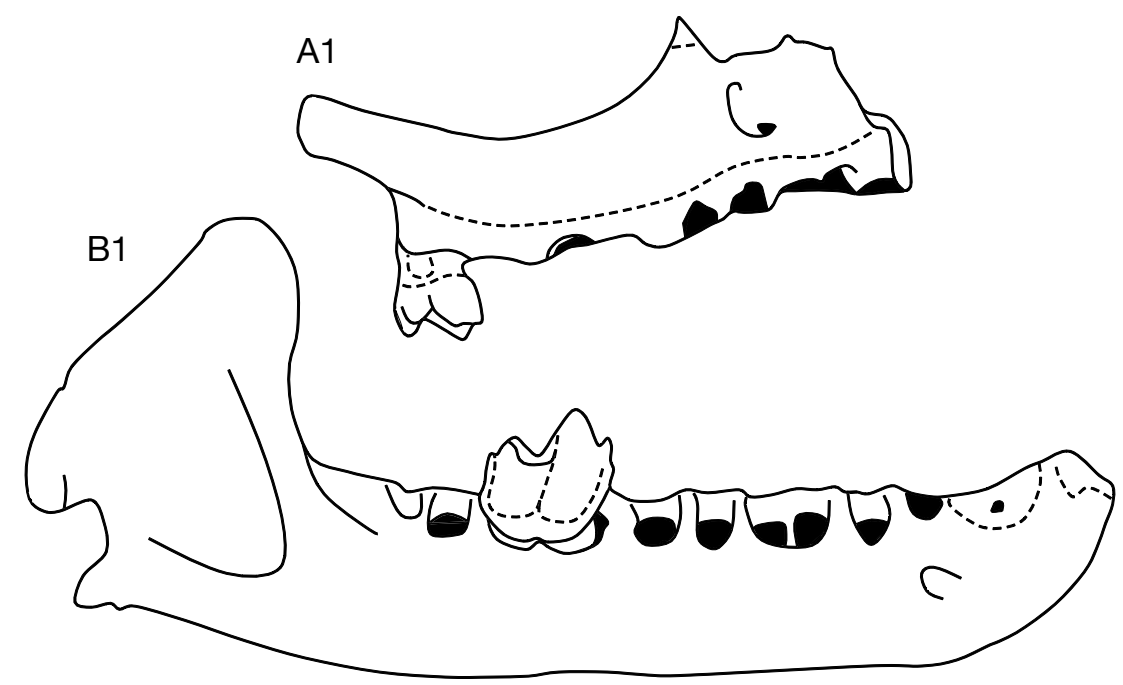

A2
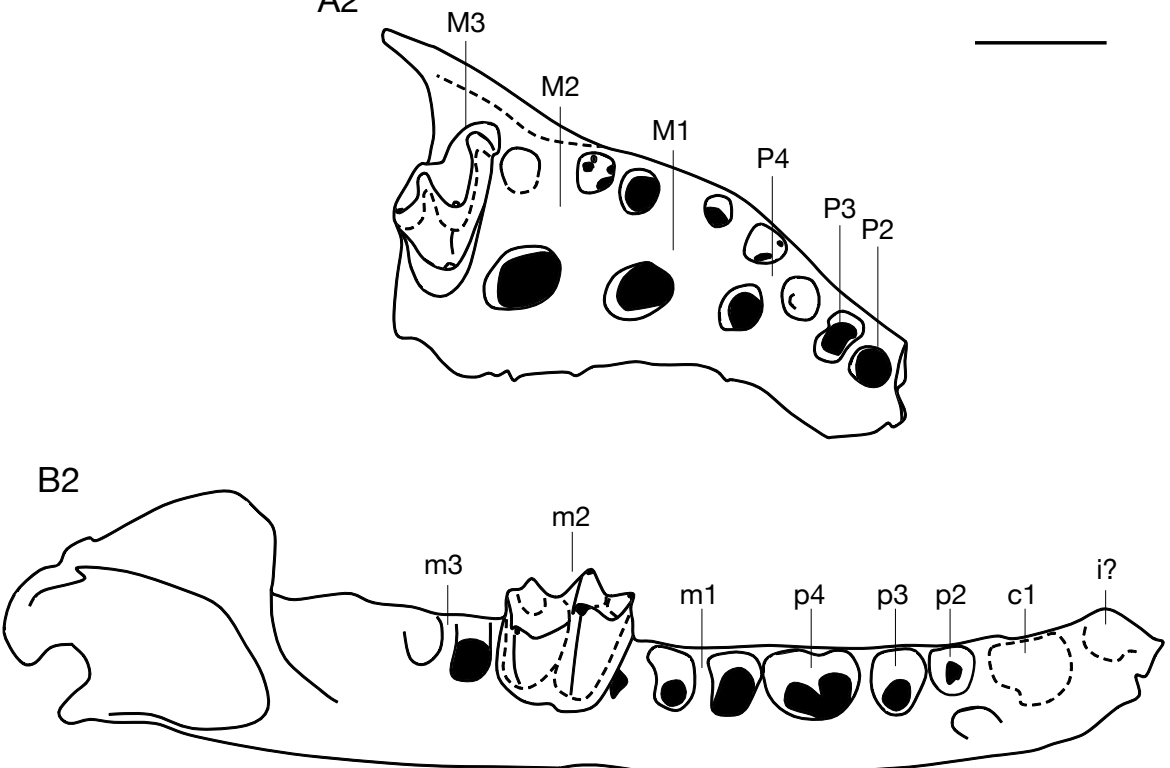

FIG. 25. - Maxillaire et dentaire de Chambinycteris pusilli Ravel n. gen., n. sp. provenant du locus 2 du Djebel Chambi dans la région de Kasserine, Tunisie: A, CBI-2-189, fragment de maxillaire droit portant une M3 en vue buccale (A1) et occlusale (A2); B, CBI-2-190, dentaire droit portant m2 en vue buccale (B1) et semi-occlusale (B2). Échelle: $1 \mathrm{~mm}$.

Chambinycteris pusilli Ravel, n. sp.

(Figs 25, 26, 27, 28, 29, 30; Tableau 10)

Holotype. - Spécimen CBI-2-189, fragment de maxillaire droit portant $\mathrm{M} 3$ et présentant les alvéoles de P2 à M2 (Figs $25 \mathrm{~A} ; 27 \mathrm{~A})$.

MATÉRIEl EXAminé. - CBI-2-204 (Figs 26C; 28C) et CBI-3016 (M1s gauches); CBI-2-190 (mandibule droite portant $\mathrm{m} 2$ Figs 25B ; 27B) ; CBI-2-196 (Figs 26A; 28A) et CBI-2-197 (C1s gauches; Figs 26B ; 28B) ; CBI-3-015 (M3 gauche; Figs 26D; 28D) ; CBI-2-206 (Figs 26E; 28E) et CBI-2-207 (c1s droites; Figs 26F ; 28F).

Localité TYPE ET ÂGE. - Chambi (CBI) loci 2 et 3, Éocène inférieur terminal-Éocène moyen basal, Djebel Chambi, situé dans la région de Kasserine, Tunisie.
ÉTYMOLOGIE. — Le nom de l'espèce vient du latin «pusilli» qui veut dire chétif, en raison de la petite taille et de l'aspect fragile de cette espèce.

DiAGNOSE. - Chiroptère ayant une formule dentaire ?/?I ; 1/1C ; $3 / 3 \mathrm{P} ; 3 / 3 \mathrm{M}$. Orbite très en avant du crâne; mandibule à processus coronoïde haut et arrondi. Canines supérieures et inférieures réduites; $\mathrm{P} 2$ uniradiculée de taille équivalente à $\mathrm{P} 3$; bord buccal de M1 bien incliné, ectolophe formant un « $W$ » bien ouvert mais comprimé buccolingualement; talon fortement projeté distolingualement muni d'un bassin très réduit; cingulum lingual épais formant un petit hypocône; M3 avec métacône bien formé, paralophe et cingulum lingual peu prononcés. p2 et p3 uniradiculées; molaires inférieures nyctalodontes avec trigonide et talonide de largeur et longueur équivalentes; entoconide faible et incliné distalement; talonide plus long mais aussi large que le trigonide. 

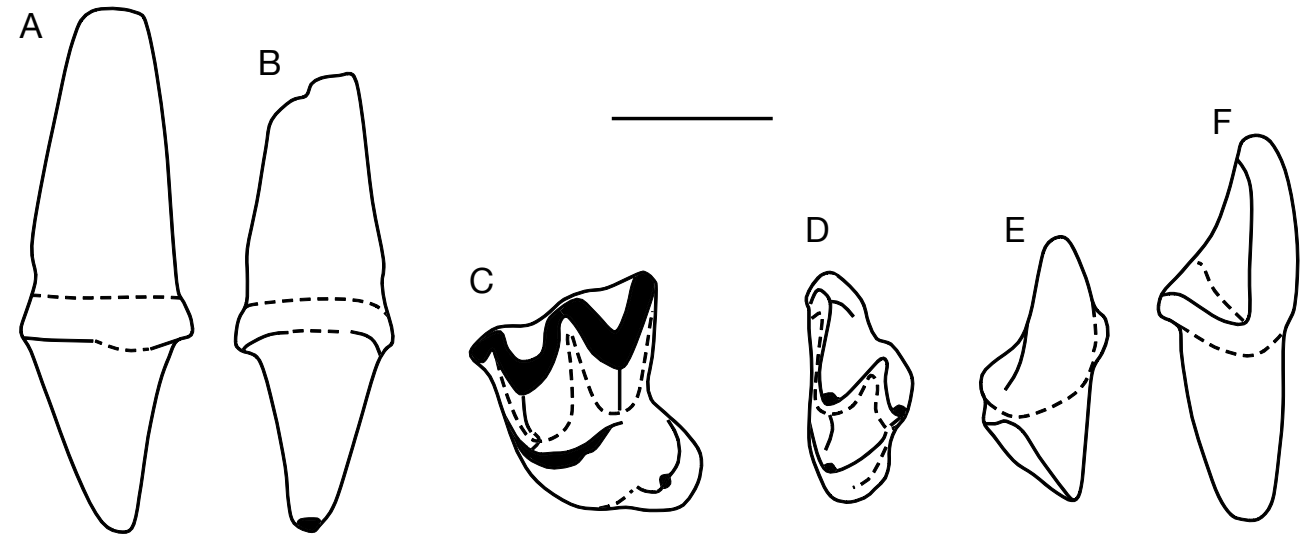

FIG. 26. - Chambinycteris pusilli Ravel n. gen., n. sp. provenant des loci 2 et 3 du Djebel Chambi dans la région de Kasserine, Tunisie: A, CBI-2-196, C1 gauche en vue buccale; B, CBI-2-197, C1 gauche en vue linguale; C, CBI-2-204, M1 gauche en vue occlusale; D, CBI-3-015, M3 gauche en vue occlusale; E, CBI-2206, c1 droite en vue buccale; F, CBI-2-207, c1 droite en vue distolinguale. Échelle: $1 \mathrm{~mm}$.

\section{DESCRIPTION}

Le maxillaire CBI-2-189 (Figs 25A; 27A) permet d'identifier la composition d'une grande partie de la rangée dentaire. Celle-ci comprend:

- une P2 monoradiculée; l'alvéole de cette dent est de taille similaire à celle de $\mathrm{P} 3$ et se positionne sur l'axe de la rangée dentaire;

- une P3, dont la présence est attestée par une alvéole. Cette alvéole présente une extension transversale et un léger étranglement central, ce qui suggère que cette dent possédait deux racines fusionnées;

- une P4 triradiculée. Les trois alvéoles sont identiques en taille. Les deux alvéoles distales sont placées sur le même axe buccolingual et la troisième est positionnée centromésialement par rapport aux deux autres;

- trois molaires (M1, M2 et M3, dont M3 est préservée sur le maxillaire) triradiculées. Les deux alvéoles buccales des M1-2s sont arrondies et de taille équivalente. La troisième, en position linguale et légèrement désaxée distalement, est la plus grande.

En vue occlusale, l'os maxillaire s'amincit fortement vers la partie mésiale, induisant ainsi une inclinaison prononcée du bord latéral. Le fragment a conservé une partie du bord externe de l'orbite. L'arcade orbitaire s'avance jusqu'au niveau de l'alvéole distale de $\mathrm{P} 4$ où elle devient fortement ascendante. De ce fait, l'orbite était développée considérablement vers l'avant du crâne. Une portion antérieure de l'arcade zygomatique est également observable. Le processus zygomatique s'incline fortement distofrontalement par rapport à la bordure ventrale tout en gardant une hauteur constante jusqu'au niveau de sa cassure. La paroi externe du maxillaire sous-jacente à l'orbite est très haute. La bordure buccale est rectiligne depuis M3 jusqu'à P2. Le foramen infraorbitaire est situé antérieurement et ventralement par rapport à l'orbite, soit au niveau de P4.

La M3 du maxillaire CBI-2-189 est similaire au spécimen isolé CBI-3-015 (Figs 26D; 28D). Ces M3s possèdent un fort développement transversal. Seule la postmétacrête est absente de l'ectolophe. Le métacône est bien formé et se positionne sur le même axe mésiodistal que le paracône. Le volume de ces deux cuspides est identique. Le parastyle forme un crochet arrondi à l'extrémité de la prémétacrête. Le mésostyle est bien projeté buccalement provocant un fléchissement de la bordure buccale de la couronne. Un paralophe émerge depuis la base du paracône et prend une direction linguale vers le protocône. Le protocône est moins élevé que le paracône et le métacône, mais il est plus long et large. La préprotocrête rejoint le large précingulum tandis que la postprotocrête gagne la base linguale du métacône au niveau de la bordure distale. Le bord lingual est arrondi sur la M3 du spécimen CBI-2-189. Sur CBI-3-015, un renflement se distingue à la base distolinguale du protocône suggérant la présence d'un cingulum lingual peu individualisé.

La canine supérieure est très simple (Figs 26A, B; 28A, B). La cuspide principale, peu élevée et arrondie au niveau de l'apex, se divise en une surface buccale convexe et une surface linguale plate. Ces deux surfaces sont séparées par des crêtes sagittales qui s'accentuent vers la base de la cuspide. La couronne est ceinturée par un cingulum épais et continu. Le cingulum est bien individualisé sur la face linguale mais s'affine très nettement côté buccal.

La compatibilité du spécimen CBI-2-204 avec le maxillaire est testée selon une superposition des spécimens en $2 \mathrm{D}$ (Fig. 29). La dent isolée présente une taille trop importante pour le maxillaire. Néanmoins, la différence de taille est peu significative et constitue la plus forte compatibilité parmi l'ensemble des molaires identifiées. M1 est très comprimée buccolingualement et possède un important talon projeté distolingualement. Le bord buccal est incliné mésiolingualement. Les deux ectoflexus sont très légèrement creusés. L'ectolophe, fortement compressé buccolingualement forme un «W » bien ouvert. L'orientation fortement distobuccale de la postmétacrête et sa longueur légèrement plus importante que les autres crêtes confèrent à l'ectolophe une dissymétrie modérée. Le parastyle est recourbé mésialement et se situe plus lingualement que le mésostyle ou le métastyle. Le mésostyle est bien déjeté sur le bord buccal. Sur la bordure mésiale, le parastyle fléchit brutalement mésiolingualement. À l'opposé, le métastyle est plus simple et effectue le prolonge- 

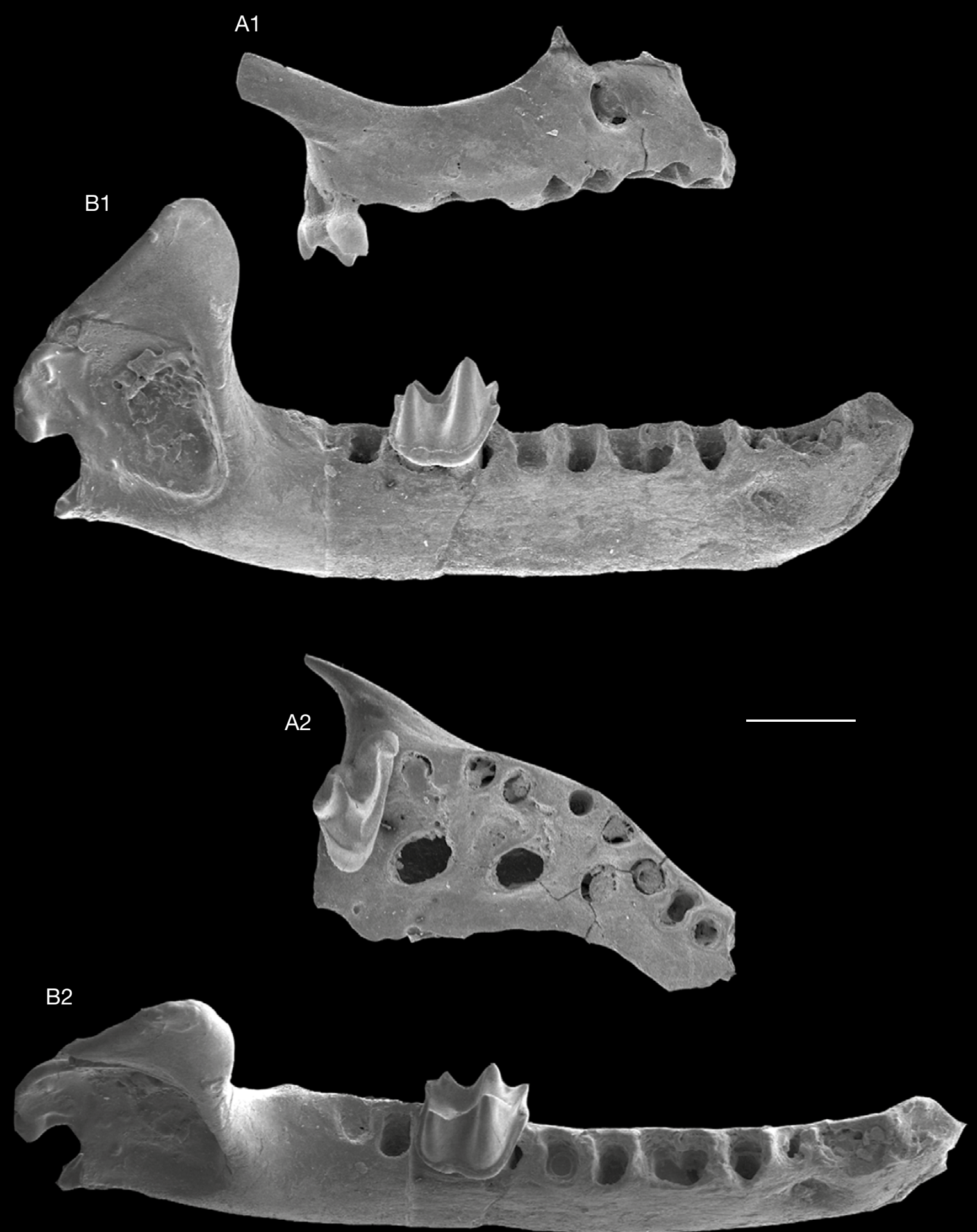

FIG. 27. - Maxillaire et dentaire de Chambinycteris pusilli Ravel n. gen., n. sp. provenant du locus 2 du Djebel Chambi dans la région de Kasserine, Tunisie: A, CBI-2-189, fragment de maxillaire droit portant une M3 en vue buccale (A1) et occlusale (A2); B, CBI-2-190, dentaire droit portant m2 en vue buccale (B1) et semi-occlusale (B2). Échelle: $1 \mathrm{~mm}$.

ment buccal de la postmétacrête. Le paracône est placé plus lingualement que le métacône. Le métacône est fortement pincé mésiodistalement mais il reste de taille équivalente au paracône. Le protocône est comprimé buccolingualement et se projette vers l'extrémité mésiale de la dent. Il est plus étiré sur l'axe mésiodistal que le paracône et le métacône. 


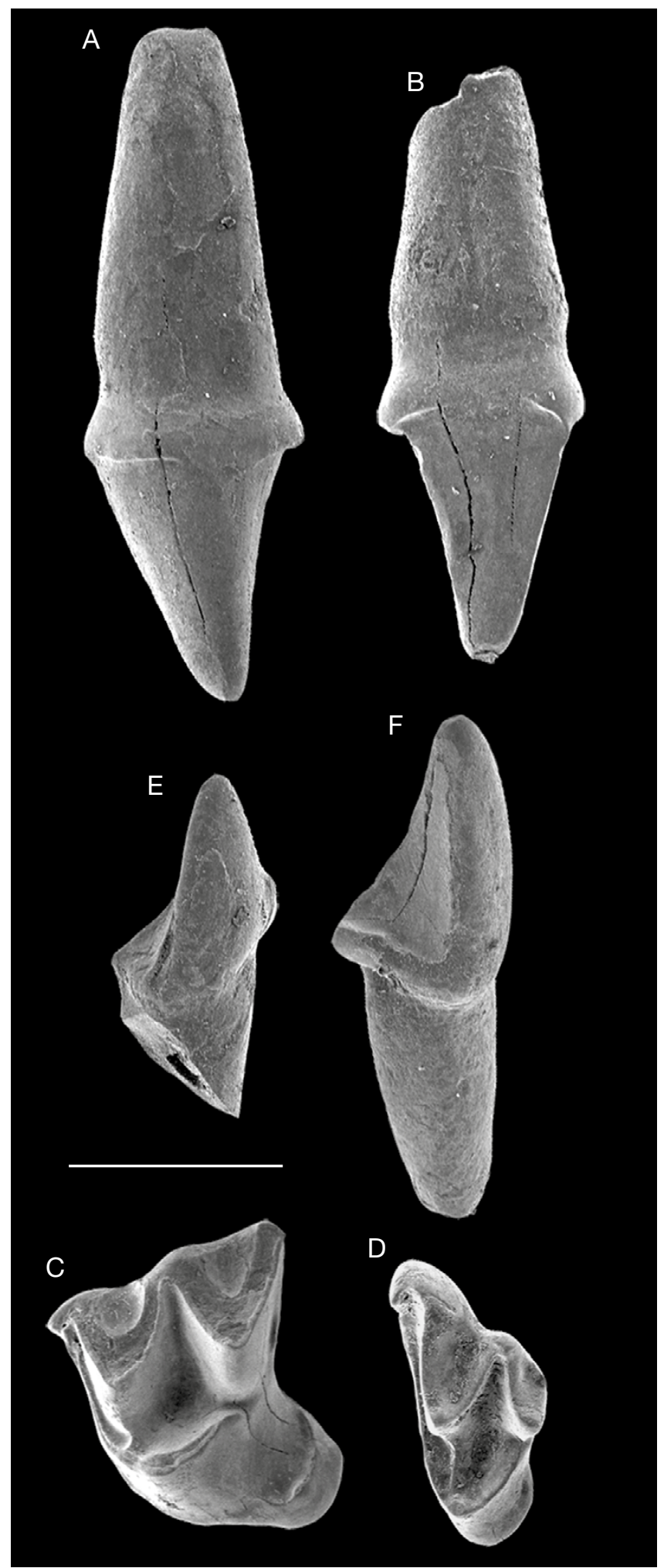

FIG. 28. - Chambinycteris pusilli Ravel n. gen., n. sp. provenant des loci 2 et 3 du Djebel Chambi dans la région de Kasserine, Tunisie: A, CBI-2-196, C1 gauche en vue buccale; B, CBI-2-197, C1 gauche en vue linguale; C, CBI-2-204, M1 gauche en vue occlusale; D, CBI-3-015, M3 gauche en vue occlusale; E, CBI-2-206, c1 droite en vue buccale; F, CBI-2-207, c1 droite en vue distolinguale. Échelle: $1 \mathrm{~mm}$.

La préprotocrête assez courte fait la jonction avec le précingulum. Le paralophe prend une direction mésiolinguale depuis la base du métacône. La postprotocrête prend une direction quasi horizontale depuis le sommet du protocône. Elle se termine au niveau d'une encoche située à la base du métacône. Le talon, fortement projeté distolingualement, est muni d'un bassin très court, ceinturé par un cingulum lingual épais. Le cingulum porte un petit hypocône qui se positionne distolingualement par rapport au protocône.

Le spécimen CBI-2-190 correspond à un dentaire quasicomplet portant une m2 (Figs 25B; 27B). L'ensemble des alvéoles sont conservées. La rangée dentaire comprend:

- une p2 monoradiculée, qui est la plus petite des dents jugales. L'alvéole est significativement plus réduite que celle de p3. Sa position est axée sur l'ensemble de la rangée;

- une p3 monoradiculée. L'alvéole de cette dent est de même largeur que celle de $\mathrm{p} 4$;

- une p4 présentant deux alvéoles fusionnées, ce qui laisse supposer que cette dent possédait deux racines;

- une série complète de molaires $(\mathrm{m} 1, \mathrm{~m} 2$ et $\mathrm{m} 3)$ toutes biradiculées et positionnées selon un même axe mésiodistal.

La branche montante de l'apophyse coronoïde forme un quasi angle droit avec le ramus horizontal. Le sommet de l'apophyse est arrondi. La fosse massétérique est très profonde et très étendue dans le sens de la hauteur. Le condyle et l'apophyse angulaire n'ont pas été préservés. La hauteur du ramus horizontal est constante et supérieure à la hauteur de la molaire. Le ramus commence à s'incurver toutefois au niveau de $\mathrm{p} 2$ et s'affine brusquement au niveau des incisives. La facette symphysaire, visible côté lingual, est inclinée distalement, soulignant l'élancement mésial du ramus. Le foramen mentonnier est visible sur le ramus entre l'alvéole de p2 et celle de la canine.

Les canines inférieures possèdent une couronne munie d'un unique tubercule (Figs 26E, F; 28E, F). La face linguodistale de ce tubercule est plane et se connecte à un très court bassin situé à la base de la couronne. À l'opposé, la face buccomésiale est beaucoup plus étendue et convexe. Les deux faces sont délimitées par une crête orientée lingualement et une crête orientée buccalement. Le cingulide forme deux petits lobes: l'un en position distolinguale et l'autre en position mésiolinguale. Le cingulide est absent sur la bordure buccale.

La molaire présente sur le dentaire $(\mathrm{m} 2)$ possède un trigonide légèrement comprimé distomésialement (Figs 25B ; 27B). La largeur du triangle mésial est similaire à celle du triangle distal. Le talonide est par contre légèrement plus allongé. Les trois cuspides du trigonide sont bien individualisées et droites. Le paraconide, la plus petite des trois cuspides du trigonide, est très légèrement inclinée vers la bordure mésiale. Le métaconide est plus redressé et plus volumineux que le paraconide. Le protoconide, dominant en taille les deux autres cuspides, présente un pincement mésiodistal modéré. Le talonide présente un schéma nyctalodonte classique: l'hypoconide et l'hypoconulide sont très réduits, et sont connectés par la postcristide, isolant ainsi l'entoconide. Le flanc buccal de l'hypoconide, tout comme celui du protoconide, est légèrement pincé mésiodistalement. La cristide oblique remonte sur le bord distal du trigonide et rejoint l'encoche laissée entre le protoconide et le métaconide. 
A

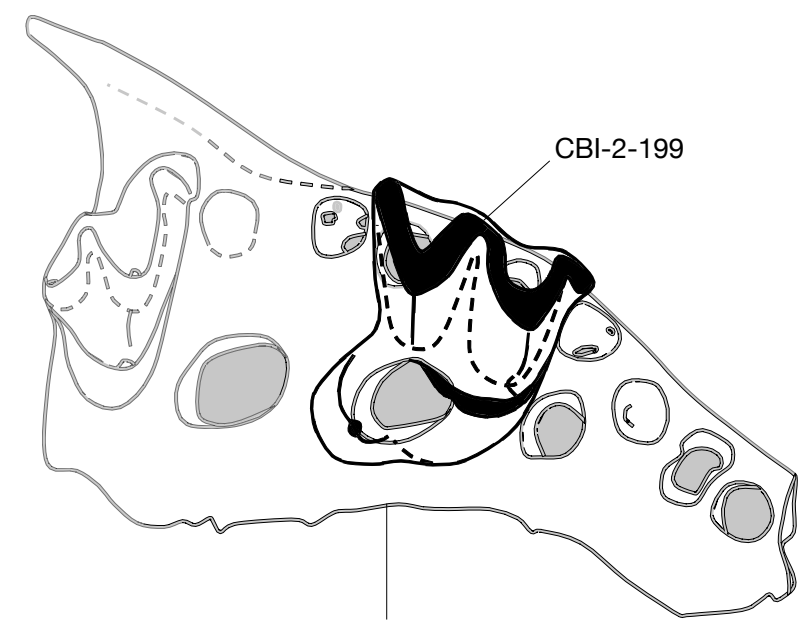

CBI-2-189

B
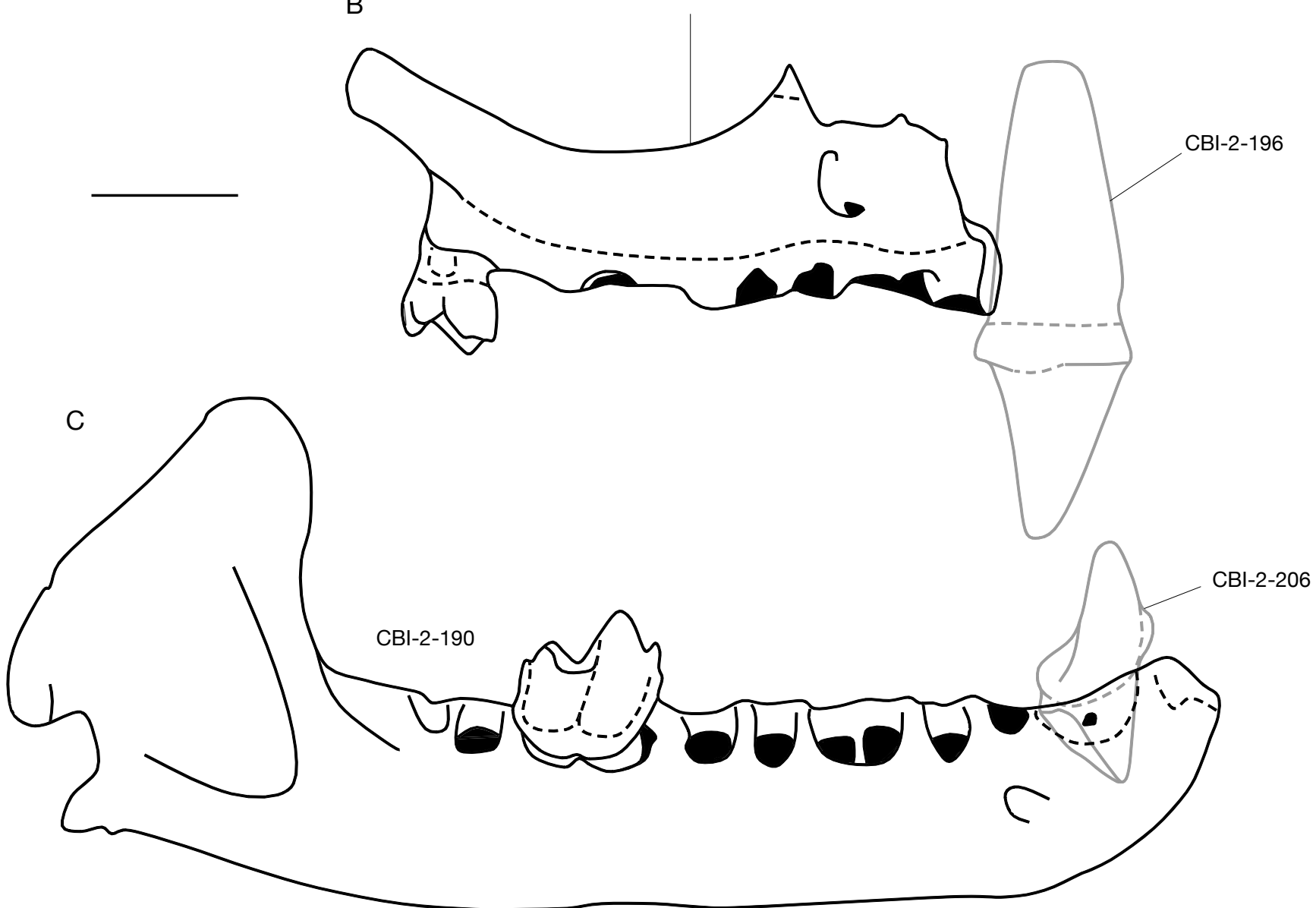

FIG. 29. - Essai de compatibilité entre les spécimens du Djebel Chambi attribué à Chambinycteris pusilli Ravel n. gen., n. sp. par superposition en 2 dimensions: A, test de compatibilité entre la M1 isolée CBI-2-204 (en miroir) et le maxillaire CBI-2-189, vue occlusale; B, test de compatibilité entre la canine supérieure isolée CBI-2-196 et le maxillaire CBI-2-189, en vue buccale; C, test de compatibilité entre la canine inférieure isolée CBI-2-206 et le dentaire CBI-2-190, en vue semi-buccale. Échelle: $1 \mathrm{~mm}$.

L'hypoconulide très réduit est positionné distalement par rapport à l'entoconide. Ce dernier est incliné distalement et forme une fine pointe dont la hauteur reste inférieure à celle du métaconide. Le cingulide cerne tout le contour de la couronne (à l'exception de la bordure linguale) en ayant une épaisseur constante.

\section{COMPARAISON ET DISCUSSION}

La composition de la rangée dentaire ( ?I, 1/1 C, 3/3 P, 3/3M) de Chambinycteris pusilli Ravel n. gen., n. sp. rappelle la condition observée chez les formes primitives de chiroptères. En effet, ces dernières présentent des rangées dentaires inférieures et supérieures complètes (seul P1 et p1 sont absentes). La 
projection verticale de la branche montante du processus coronoïde est également courante chez les formes primitives comme par exemple chez Icaronycteris, Archaeonycteris, Hassianycteris Smith \& Storch, 1981, et Palaeochiropteryx Revilliod, 1917. En revanche, C. pusilli Ravel n. gen., n. sp. présente également de nombreuses différences qui ne correspondent pas à ce que l'on attend d'une forme primitive:

- P3 possède deux racines fusionnées;

- M3 est beaucoup plus développée et ne possède pas de cingulum bien individualisé;

- les deux ectoflexus sont très faibles;

- sur M1, l'ectolophe est plus ouvert mais comprimé dans le sens buccolingual; le mésostyle est déjeté sur la bordure buccale; le bord buccal est fortement incliné; le protocône est comprimé buccolingualement; la présence d'un petit hypocône; le talon est fortement projeté distolingualement; - p3 possède une seule racine;

- sur $\mathrm{m} 2$, l'hypoconulide est très réduit, sans déplacement buccal, et son trigonide est comprimé mésiodistalement.

Prenant en considération ces différences, il apparaît évident que Chambinycteris pusilli Ravel n. gen., n. sp. s'écarte des formes primitives et possède plutôt une morphologie moderne dont les affinités avec une famille actuelle restent à déterminer. Le matériel référé à $C$. pusilli Ravel n. gen., n. sp. montre dans sa globalité certaines affinités avec les deux grands ensembles de chiroptères modernes que sont les Rhinolophoïdea et les Emballonuroïdea.

Chez C. pusilli Ravel n. gen., n. sp., M1 possède des caractéristiques communes avec le grand ensemble des Rhinolophoïdea: l'ouverture de l'ectolophe, la présence d'un talon bien développé, l'aspect comprimé de la molaire, le double ectoflexus, la projection du mésostyle. Elle s'en écarte malgré tout par la forte inclinaison du bord buccal (partagé uniquement avec Hipposideros [Pseudorhinolophus] africanum Ravel, n. sp. [voir plus haut]), l'élongation de la postprotocrête, la projection très distale du talon et la présence d'un faible hypocône. Ces différences s'ajoutent à celles rencontrées avec le reste du matériel:

- le maxillaire possède une $\mathrm{P} 3$ et une $\mathrm{P} 2$, toutes deux placées sur l'axe de la rangée dentaire. La P2 est absente chez les rhinolophoïdes actuels et fossiles. Quand elle est présente, la P3 uniradiculée est très réduite et déjetée sur le bord buccal chez les Rhinolophoïdea;

- M3 présente un développement et une morphologie similaire à celle des Rhinolophidae. Seuls le paralophe et l'absence d'un fort rétrécissement mésio-distal de la partie linguale de la couronne diffèrent;

- parmi les Rhinolophoïdea, les Rhinolophidae et les Hipposideridae fossiles possèdent trois prémolaires inférieures. Cependant, chez ces derniers, p3 est conservée sous une forme vestigiale, et elle est déjetée sur le bord buccal. Le dentaire de C. pusilli Ravel n. gen., n. sp. présente une alvéole de p3 plus importante que celle de p2. La p3 parait donc bien développée et n'est pas déjetée sur le bord buccal comme chez les rhinolophoïdes;

- la position très antérieure de l'orbite et la position isolée du foramen infraorbitaire diffèrent de ce qu'il est possible d'observer chez les rhinolophoïdes (une orbite plus reculée et un foramen infraorbitaire plus proche de l'orbite);

- la bordure buccale du maxillaire est rectiligne, ce qui diffère de la forte inclinaison frontomésiale observée chez les rhinolophoïdes actuels;

- le dentaire possède un ramus horizontal assez haut, une apophyse coronoïde très élevée et peu allongée. Seul les Hipposideridae possèdent une branche montante très haute. Cependant l'apophyse coronoïde est plus pointue et inclinée distalement;

- m2 diffère des molaires inférieures des rhinolophoïdes par son trigonide comprimé mésiodistalement et par l'inclinaison distale de l'entoconide.

Certaines ressemblances de l'ensemble du matériel existent entre Chambinycteris pusilli Ravel n. gen., n. sp. et les Emballonuroïdea, et plus particulièrement les Emballonuridae actuels: - la forte inclinaison de la bordure buccale de M1 est courante chez les Emballonuridae;

- le développement du talon très prononcé est également une caractéristique que l'on retrouve chez les Emballonuridae et chez les Nycteridae;

- la position très avancée de l'orbite;

- la hauteur importante du processus coronoïde par rapport

à la hauteur de la rangée dentaire;

- l'inclinaison distale de l'entoconide;

- la compression mésiodistale du trigonide.

Chambinycteris pusilli Ravel n. gen., n. sp. possède une P2 et une P3 dont les tailles semblent équivalentes. Les emballonuroïdes actuels ont perdu leur P3, et en outre, P2 est souvent réduite voir absente (en particulier chez les Nycteridae). Au niveau de la rangée inférieure, le taxon tunisien présente les trois prémolaires avec une p2 qui semble légèrement plus importante que p3. Les Emballonuridae et les Nycteridae actuels possèdent uniquement deux prémolaires: chez les Nycteridae, la p4 est très réduite avec une couronne à développement transversal par rapport à la rangée dentaire, la p3 est absente, et la p2 est plus imposante que la p4; chez les Emballonuridae actuels, la p3 est également perdue, la p4 est cependant mieux développée et supérieure en taille à la p2. La formule dentaire de C. pusilli Ravel n. gen., n. sp. se rapproche d'avantage des formes fossiles emballonuroïdes qui présentent des rangées dentaires plus complètes, en particulier les genres Vespertiliavus et Tachypteron qui conservent des prémolaires intermédiaires, supérieures et inférieures (Sudre 1979; Legendre 1980; Sigé 1990; Marandat et al. 1993; Storch et al. 2002; Smith et al. 2012). Cependant les différences entre le taxon tunisien et les emballonuroïdes sont nombreuses:

- chez Chambinycteris pusilli Ravel n. gen., n. sp., M1 présente des ectoflexus très faiblement creusés, conférant à la dent un bord buccal quasi rectiligne;

- la morphologie de l'ectolophe diffère des Emballonuridae par son ouverture et sa symétrie plus accentuées et des Nycteridae par sa compression buccolinguale;

- la morphologie du talon diffère significativement de la morphologie du talon des molaires supérieures des Emballonuroïdea en général, et ce par sa forte inclinaison linguale, 

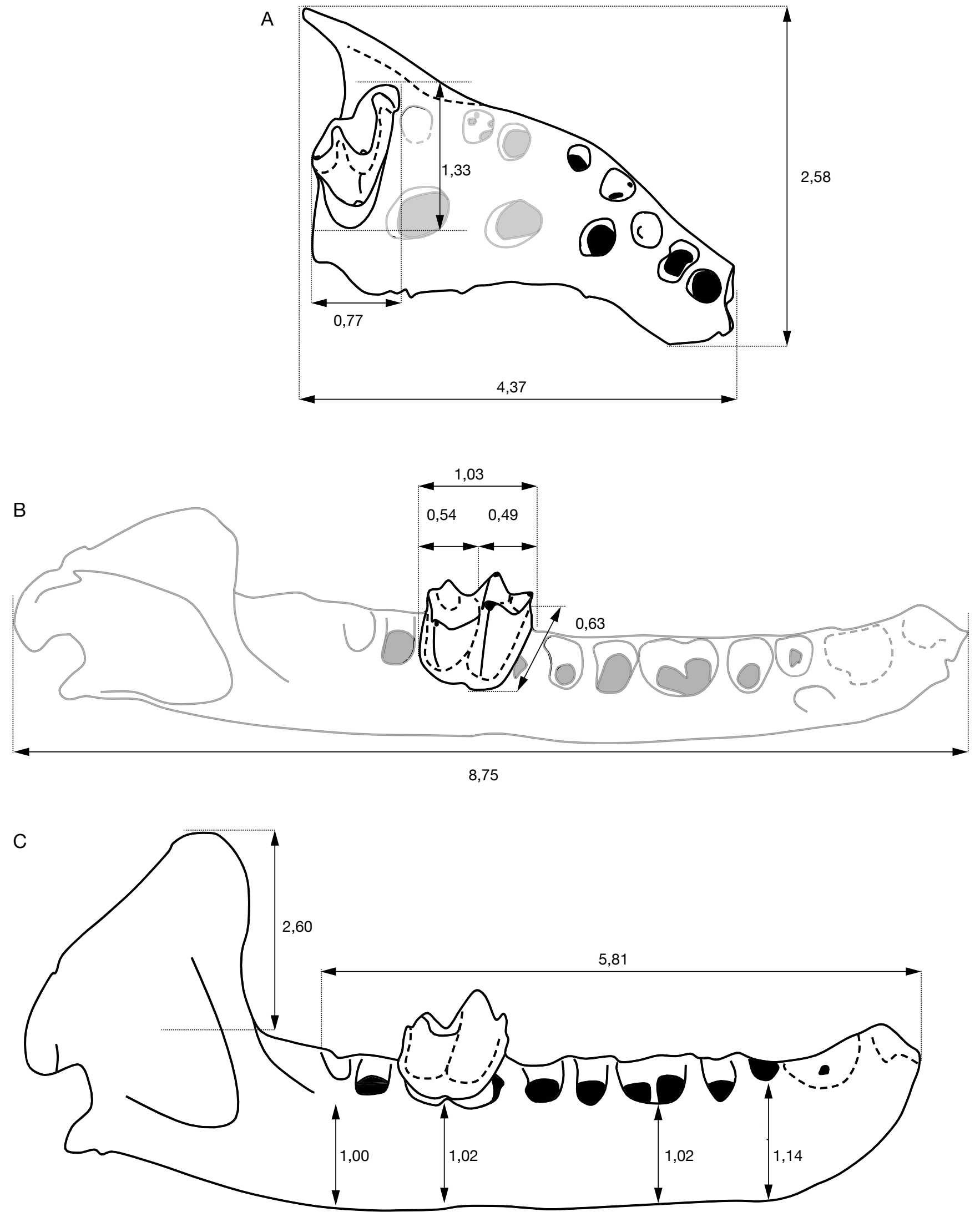

FIG. 30. - Mesures effectuées sur le maxillaire (CBI-2-189) et le dentaire (CBI-2-190) de Chambinycteris pusilli Ravel n. gen., n. sp. Les mesures sont données en millimètres $(\mathrm{mm})$. 
la réduction du bassin cerné par un cingulum épais mais de faible extension;

- si le développement de M3 de C. pusilli Ravel n. gen., n. sp. est proche du développement des M3s des Emballonuridae en général, la présence d'un paralophe et d'une ébauche de cingulum lingual sont des différences significatives qui les séparent; - l'aspect du dentaire de C. pusilli Ravel n. gen., n. sp. est singulier comparé à celui des Emballonurö̈dea, notamment par la hauteur relativement importante et homogène du ramus horizontal, la branche montante relativement plus haute et droite ainsi que le processus coronoïde plus arrondi;

- au niveau des molaires inférieures, la $\mathrm{m} 2$ de $C$. pusilli Ravel n. gen., n. sp. présente un trigonide et un talonide de dimensions équivalentes et un entoconide modéré très légèrement incliné distalement. Chez les Nycteridae les molaires inférieures ont un talonide nettement plus réduit que le trigonide, l'entoconide est très réduit voire absent et il se situe près de la base du métaconide. Chez les Emballonuridae, c'est le talonide qui est plus large et long que le trigonide, l'entoconide est élevé et fortement incliné distalement.

- Les canines supérieures et inférieures de C. pusilli Ravel n. gen., n. sp. montrent une morphologie très réduite et simple que l'on ne retrouve pas chez les Emballonuroïdes.

Chambinycteris pusilli Ravel n. gen., n. sp. montre une morphologie qui combine à la fois des caractères observés chez les rhinolophoïdes et d'autres observés plus couramment chez les emballonuroïdes. Cette combinaison a déjà été relevée dans le Quercy avec la famille des Mixopterygidae (Maitre et al. 2008). Cette famille est documentée avec un abondant matériel provenant uniquement du Sud Ouest de la France depuis la fin de l'Éocène moyen (MP13, Saint Maximin) jusqu'à l'Oligocène supérieur (MP25, l'Escoufle). Les Mixopterygidae regroupent deux genres (Mixopteryx et Carcinipteryx) incluant à eux deux, quatre espèces. La famille est définie par un ensemble de caractères qui differe cependant radicalement de la morphologie de Chambynicteris pusilli Ravel n. gen., n. sp. :

- l'ectoflexus relativement profond entre le parastyle et le mésostyle;

- la projection du talon à dominance distale;

- l'extension du bassin du talon (le bassin du talon est très étendu sur Mixopteryx, il l'est beaucoup moins sur les dents de Carcinipteryx);

- le développement de M3 plus réduite (uniquement pour Mixopteryx);

- entoconide médian et droit;

- absence de p3;

- le trigonide des molaires inférieures plus ouvert et de largeur inférieure à celle du talonide;

- les canines inférieures et supérieures recourbées distalement et possédant un cingulum mieux défini;

- les cuspides du trigonide moins individualisées;

- l'absence d'hypocône;

- l'inclinaison et la hauteur moins importante de la branche montante du processus coronoïde.

Le regroupement du matériel dentaire attribué à Chambinycteris pusilli Ravel n. gen., n. sp. est basé en grande partie sur sa petite taille qui ne peut correspondre à aucun autre taxon de la localité. Les tests de compatibilité par superposition en $2 \mathrm{D}$ restent convaincants et permettent de mieux cerner la morphologie de ce taxon. Les comparaisons montrent l'association de caractères que l'on retrouve pour certains chez les Emballonuroïdea et pour d'autres chez les Rhinolophoïdea, et plus précisément chez les formes les plus primitives des ces grands ensembles. Ce constat peut suggérer l'appartenance de Chambinycteris Ravel, n. gen. à un groupe souche encore non identifié, et qui précèderait la divergence de ces grands groupes.

\section{PHYLOGÉNIE DES RHINOLOPHOIDEA}

\section{MATÉRIEL ET MÉTHODE \\ Sélection des caractères}

Le but de cette analyse cladistique est d'évaluer au sein du clade regroupant Hipposideridae et Rhinolophidae, la position phylogénétique des espèces fossiles ?Palaeophyllophora tunisiensis Ravel, n. sp. et Hipposideros (Pseudorhinolophus) africanum Ravel, n. sp. découverts à Chambi. Ces deux espèces sont documentées uniquement par des dents isolées. C'est pourquoi 54 caractères ont été choisis en relation avec la morphologie dentaire et seulement six avec celle de la mandibule (Annexe 1). Les caractères ont été repris de Ravel et al. (2014). Toutefois, pour mieux discriminer les autres espèces échantillonnées et tester la robustesse des nœuds déduits de l'anatomie dentaire, 44 caractères crâniens et postcrâniens définis dans l'analyse cladistique de Hand \& Kirsch (2003) ont été ajoutés à la matrice initiale.

\section{Sélection des taxons}

Un groupe externe a été choisi afin de permettre une polarisation des caractères a posteriori selon la méthode de Watrous \& Wheeler (1981). Il est constitué de trois taxons primitifs découverts dans l'Éocène européen: Icaronycteris menui, et Archaeonycteris brailloni proviennent tout deux de l'Éocène inférieur du Bassin de Paris en France (Mutigny et Avenay, MP8+9); Palaeochiropteryx tupaiodon Revilliod, 1917 provient de l'Éocène moyen de Messel (MP11) en Allemagne. La sélection des taxons constituant le groupe interne a été réalisée de façon à introduire des formes fossiles et actuelles appartenant aux Hipposideridae et aux Rhinolophidae. En plus de ?Palaeophyllophora tunisiensis Ravel, n. sp. et Hipposideros (Pseudorhinolophus) africanum Ravel, n. sp. récoltés à Chambi, l'échantillonnage des taxons fossiles inclut plusieurs espèces appartenant aux mêmes genres, toutes provenant de localités tertiaires européennes: Palaeophyllophora quercyii Revilliod, 1917 et $P$. oltina trouvés dans les remplissages karstiques de l'Éocène moyen et supérieur du Quercy (Sud Ouest de la France; MP18 pour P. oltina et MP16 à MP19 pour P. quercyi); Hipposideros (Pseudorhinolophus) schlosseri Revilliod, 1917 documenté depuis l'Éocène moyen (MP16) jusqu'à l'Oligocène inférieur (MP22) du Quercy, et $H$. (Ps.) bouziguensis décrit des phosphorites de Bouzigues (Sud de la France) et daté du Miocène inférieur. Les Rhinolophidae fossiles comprennent Vaylatsia prisca Revilliod, 1920 provenant des poches karstiques du Quercy depuis le niveau MP17a 


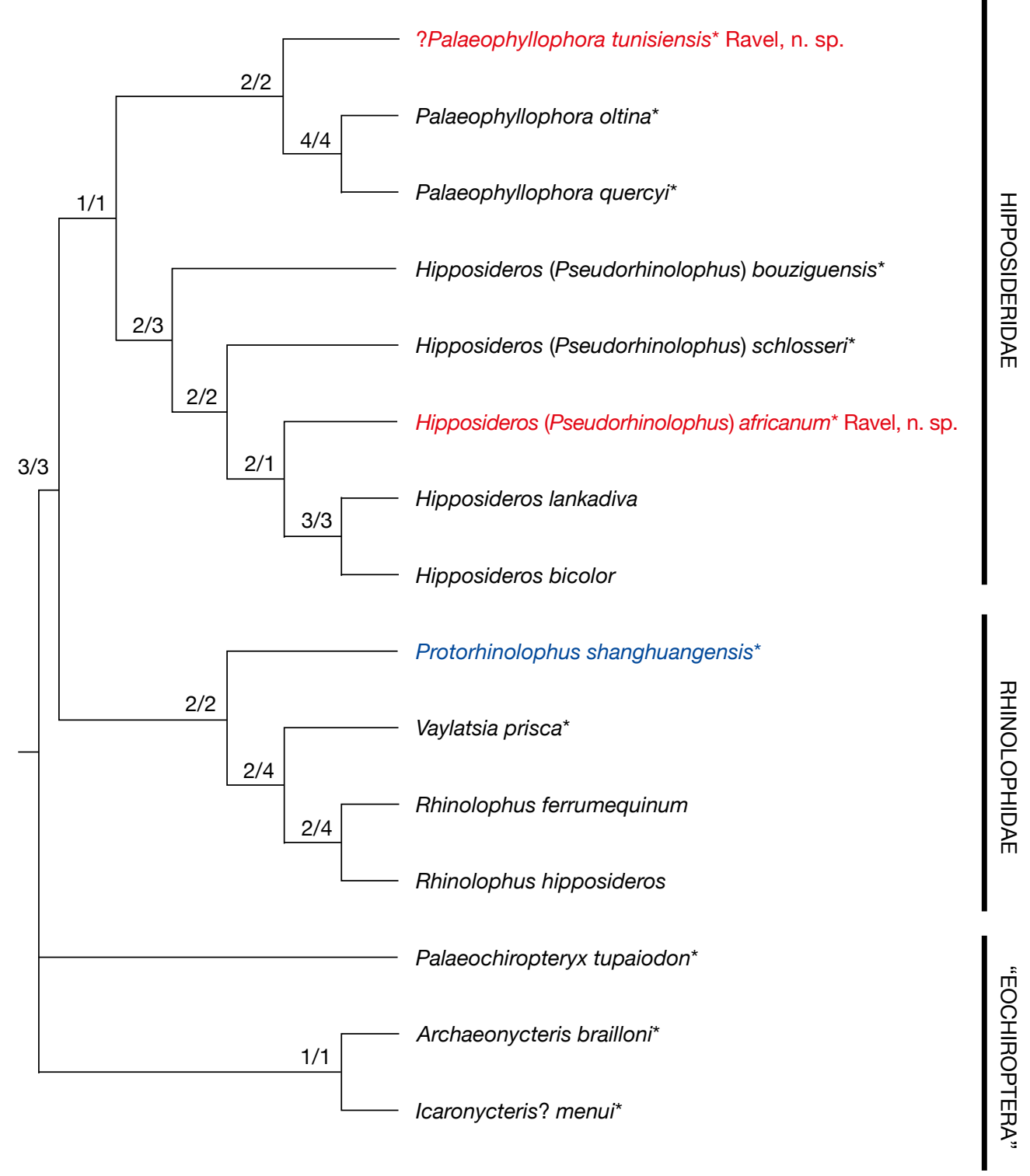

FIG. 31. - Phylogénie incluant les Rhinolophoidea. Arbre le plus parcimonieux (129 pas, I.C. = 0.488 et I.R. =0.683) issue de l'analyse cladistique en «Branch and Bound " réalisée à partir de 61 caractères dentaires et mandibulaires. Un seul arbre présentant une topologie similaire (165 pas, I.C. $=0.593$ et I.R. $=0.683)$ est également obtenu à partir de l'analyse utilisant à la fois les caractères dentaires, crâniens et postcrâniens. Les caractères crâniens et postcrâniens sont issus de Hand et Kirsch, 2003. Les indices de Bremer sont indiqués en haut à gauche de chaque nœud. L'indice le plus à gauche correspond à la robustesse du nœud obtenue à partir de l'analyse basée uniquement sur les caractères dentaires et mandibulaires, celui de droite correspond à la robustesse du nœud obtenue après l'analyse comprenant l'ensemble des caractères dentaires, crâniens et postcrâniens. Les noms d'espèces suivis du signe * indiquent une espèce fossile. Le taxon en bleu représente l'espèce provenant de Shanghuang (i.e., Protorhinolophus shanghuangensis Ravel, Marivaux, Qi, Wang \& Beard, 2014). Les espèces en rouge correspondent aux taxons provenant de Chambi, Tunisie.

(Éocène supérieur) jusqu’au niveau MP23 (Oligocène inférieur), et Protorhinolophus shanghuangensis Ravel, Marivaux, Qi, Wang \& Beard, 2014, provenant des karsts de Shanghuang en Chine et datés du milieu de l'Éocène moyen (Ravel et al. 2014). Les Hipposideridae actuels sont représentés par les espèces Hipposideros bicolor Temminck, 1834 et H. lankadiva Kelaart, 1850, qui sont communes dans le Sud Est de l'Asie (Hill et al. 2009). Les Rhinolophidae actuels comprennent Rhinolophus ferrumequinum Schreber, 1774 et Rhinolophus hipposideros Bechstein, 1800. Ces deux espèces sont très répandues de l'Afrique du Nord jusqu'à l'Asie de l'Est, en passant par l'Europe.

\section{Analyse de parcimonie}

Étant donné le faible nombre de taxon (15), l'analyse a été effectuée en utilisant l'algorithme de recherche "Branch and Bound" (Annexe 1). La méthode et les logiciels utilisés sont expliqués dans la partie Matériel et méthodes.

\section{RÉSULTATS}

Les recherches ont fourni un seul arbre (Fig. 31) de 129 pas, avec un indice de consistance (I.C.) de 0,488 et un indice de rétention (I.R.) de 0,683. La même topologie a été obtenue en incluant les caractères crâniens et postcrâniens. L'arbre obtenu possède 165 pas, avec un I.C. de 0,593 et un I.R. 
de 0,707 (Fig. 31). La topologie qui en résulte représente les Rhinolophidae et les Hipposideridae comme deux groupes monophylétiques bien distincts.

Les Hipposideridae forment un clade constitué des Palaeophyllophora, des deux espèces actuelles du genre Hipposideros, et des espèces du sous-genre Pseudorhinolophus. Le clade des Hipposideridae est supporté par une synapomorphie exclusive et non ambigüe qui est l'extension de la postprotocrista, laquelle ne rejoint ni le cingulum lingual ni le postcingulum $\left(48^{1}\right.$ : I.C. $=1 ;$ I.R. = 1). Deux autres synapomorphies non exclusives caractérisent les Hipposideridae: l'absence d'un cingulum buccal sur C1 (291: I.C. $=0,50$; I.R. = 0,83), également présent chez $P$. oltina, et l'absence d'un lobe mésiobuccal sur P4 (35': I.C. = 1; I.R. = 1) qui est ambigüe pour les deux taxons de Chambi (leur P4 est inconnue). Au sein des Hipposideridae, les espèces du genre Palaeophyllophora forment un groupe naturel $($ Bremer $=2 / 2)$ bien soutenu par deux synapomorphies non homoplasiques et non ambigües: la largeur du talonide inférieure à celle du trigonide (241 : I.C. $=0,66$; I.R. $=0,66)$, et la protofossa n'ayant pas d'extension buccale (461: I.C. = 1; I.R. = 1). Ce clade se branche à la base de tous les autres Hipposideridae. Le genre Hipposideros sensu lato $($ Bremer $=2 / 3)$ se définit par l'absence d'une $\mathrm{p} 3$ vestigiale $\left(12^{1}\right.$ : I.C. $=1$; I.R. = 1). Néanmoins, cette caractéristique ne peut pas être démontrée chez $H$. (Ps.) africanum Ravel, n. sp. Le sous-genre Pseudorhinolophus apparaît totalement paraphylétique, comprenant une succession de branchements à la base des Hipposideros. Hipposideros (Ps.) bouziguensis se place à la base du clade, suivi de $H$. (Ps.) schlosseri et enfin de H. (Ps.) africanum Ravel, n. sp. Le genre Hipposideros sensu stricto, constitué des deux espèces actuelles (Hipposideros lankadiva et Hipposideros bicolor), forme le groupe couronne des Hipposideridae. Ce dernier se définit par l'absence d'un rétrécissement mésiodistal des molaires supérieures à mi largeur de la couronne $\left(37^{1}\right.$ : I.C. = 1; I.R. = 1). Les Rhinolophidae incluent les fossiles Protorhinolophus shanghuangensis et Vaylatsia frequens Maitre, 2014, ainsi que les deux espèces actuelles Rhinolophus ferrumequinum et $R$. hipposideros. Le groupe frère des Hipposideridae se caractérise ici par: l'apophyse coronoïde basse $\left(1^{1}\right.$; I.C. = 1 ; I.R. = 1$)$, la forte extension transversale du bassin de P4 $\left(33^{1}\right.$; I.C. $=1$; I.R. = 1$)$, et le cingulum lingual discontinu qui présente cependant une convergence avec $H$. (Ps.) africanum Ravel, n. sp. (521 : I.C. = $0,50 ;$ I.R. $=0,75)$. L'agencement des taxons qui constituent le clade des Rhinolophidae est similaire aux résultats obtenus d'une précédente analyse (Ravel et al. 2014). Le taxon de Shanghuang (Protorhinolophus) apparaît comme le plus basal des Rhinolophidae. Puis Vaylatsia frequens se positionne à la base du groupe couronne formé des deux espèces actuelles.

\section{DisCUSSION SUR LE GENRE PALAEOPHYLLOPHORA}

Le genre Palaeophyllophora semble être le premier témoin de la famille des Hipposideridae à apparaître dans les faunes de chiroptères éocènes. En effet, cet ensemble hipposidéride est attesté dès l'Éocène moyen (MP13) dans le Quercy et à Saint Maximin (Gard) avec un matériel qui demeure toutefois trop fragmentaire pour pouvoir en identifier l'espèce (Sigé
1997). Une autre forme a été reconnue à Cuzal (MP13) par une unique P4 (Maitre 2014). L'ensemble Palaeophyllophora inclut au total cinq espèces (Maitre 2014). Toutes ces espèces se répartissent en cinq lignées qui perdurent jusqu'à l'Oligocène inférieur (MP23; Sigé 1978; Maitre 2014). Les résultats de cette analyse cladistique semblent confirmer la présence d'une nouvelle espèce de Palaeophyllophora dans l'Éocène inférieur terminal - Éocène moyen basal de l'Afrique du Nord. Cette nouvelle occurrence vieillit l'âge de l'apparition du genre dans le registre fossile et élargit également son aire de répartition. Les Palaeophyllophora se distinguent parmi les Hipposideridae par une morphologie dentaire très primitive qui se traduit par une forte modification de la dilambdodontie de l'ectolophe, la conservation d'une $\mathrm{p} 3$ vestigiale, et le schéma nécromantodonte du talonide des molaires inférieures. Certains de ces caractères sont accentués chez ?P. tunisiensis Ravel, n. sp. lui conférant une morphologie qui le rapproche d'avantage des formes primitives ("Eochiroptera») telles Icaronycteris et Archaeonycteris. À cela s'ajoute d'autres caractères qui marquent un stade plus primitif pour le taxon tunisien par rapport aux formes européennes: la forte épaisseur du cingulum lingual, le développement du protocône, et le talonide légèrement plus long. En considérant l'ancienneté et la morphologie plus archaïque de ?P. tunisiensis Ravel, n. sp. par rapport aux formes européennes, on peut donc envisager que l'ensemble Palaeophyllophora témoigne d'une migration depuis l'Afrique du Nord vers l'Europe du Sud à l'Éocène moyen.

\section{DISCUSSION SUR LE GENRE HIPPOSIDEROS}

Le genre Hipposideros est un vaste ensemble actuel regroupant une grande diversité de morphologie (par ex. : Hill \& Smith 1984; Koopman 1994; Simmons \& Conway 2003). Dans le registre fossile, le genre se divise en deux sous-genres: Pseudorhinolophus, exclusivement documenté depuis l'Éocène moyen jusqu'au Miocène en Europe (Sigé 1968, 1990 ; Maitre 2014), et Brachipposideros présent dans l'Oligocène supérieur et le Miocène d'Europe (Sigé 1968; Sigé et al. 1991), dans l'Oligocène de Taqah au Sultanat d'Oman (Sigé et al. 1994), et au Miocène en Europe et en Australie (Sigé et al. 1982; Hand 1998a, b; Hand \& Kirsch 2003). Hipposideros (Pseudorhinolophus) représente le second genre d'Hipposideridae de l'Éocène du Quercy (Sigé 1966, 1978, 1990, 1995; Sigé \& Legendre 1983; Maitre 2014). Il constitue un ensemble d'espèce très courant dans le Quercy qui est attesté depuis la fin de l'Éocène moyen (MP16) jusque dans le Néogène. Hipposideros (Pseudorhinolophus) comprend six espèces constituant quatre lignées (Maitre 2014). Une septième espèce semble être identifiée dans la localité de Chamblon (MP13) et déterminerait une cinquième lignée, mais le trop faible matériel ne permet pas de cerner son implication dans la phylogénie. Selon les résultats de l'analyse cladistique, le genre Hipposideros apparaît paraphylétique (Fig. 31). Ce type de topologie ne permet pas de statuer sur les relations de parenté entre $H$. (Ps.) africanum Ravel, n. sp. et les formes du Quercy. Le taxon africain semble tout de même se rapprocher des espèces actuelles d'Hipposideros par une morphologie significativement plus dérivée que les Palaeophyllophora contemporains. 


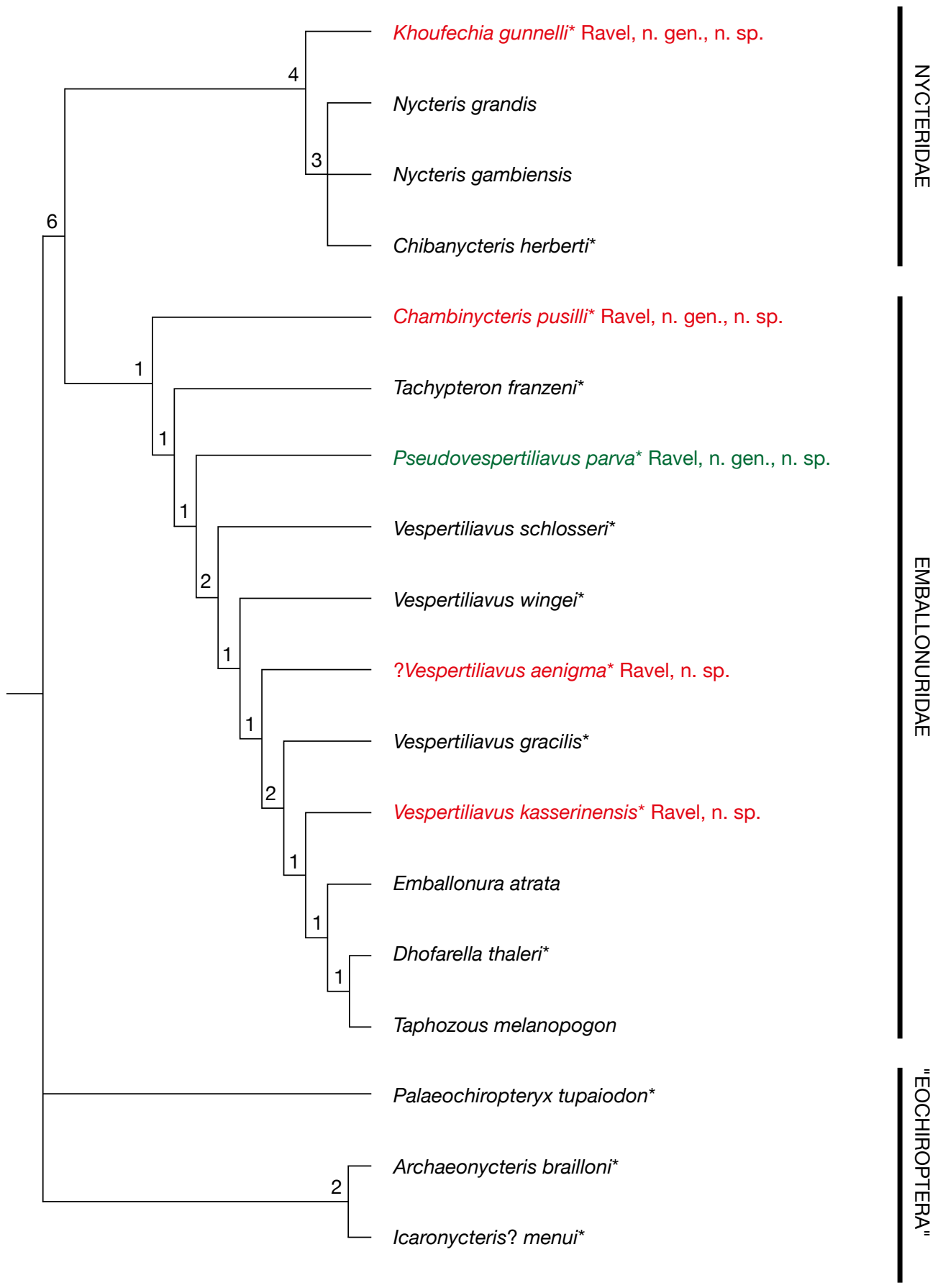

FIG. 32. - Phylogénie incluant les Emballonuroidea. Consensus strict des trois arbres les plus parcimonieux (132 pas, I.C. $=0.576$ et I.R. $=0.743)$ issus d'une recherche en «Branch and Bound » réalisée à partir de 65 caractères dentaires et mandibulaires. Les indices de Bremer sont indiqués en haut à gauche de chaque nœud. Les noms d'espèces suivis du signe * indiquent les espèces fossiles. Les noms en rouge correspondent aux taxons découverts à Chambi en Tunisie. Le taxon en vert provient du niveau HGL50 et HGL50' du Glib Zegdou en Algérie.

La présence de cette nouvelle espèce dans l'Éocène inférieur terminal-Éocène moyen basal recule considérablement l'âge d'apparition du genre Hipposideros dans le registre fossile. Il élargit également le champ de répartition du groupe Pseudorhinolophus jusque-là documenté uniquement en Europe. Si on considère la hiérarchisation des positions entre les différents Pseudorhinolophus, l'espèce $H$. (Ps.) africanum Ravel, n. sp. paraît la plus dérivée, la plus basale étant l'espèce $H$. bouz- iguensis, laquelle paradoxalement est l'espèce la plus récente selon le registre fossile. Si un événement de dispersion entre l'Afrique du Nord et le sud de l'Europe semble évident, le sens de cette dispersion ne peut être déduit directement de la phylogénie. Seule l'ancienneté du taxon africain par rapport aux formes européennes soutiendrait une migration de l'Afrique du Nord vers le sud de l'Europe, comme cela semble être le cas pour les Palaeophyllophora. 


\section{PHYLOGÉNIE DES EMBALLONUROIDEA}

\section{MATÉRIEL ET MÉTHODE \\ Sélection des caractères}

Les faunes de Chambi et du Glib Zegdou sont composées de nombreuses formes ayant de fortes affinités morphologiques avec les Nycteridae (i.e., Khoufechia gunnelli Ravel n. gen., n. sp.) et les Emballonuridae (i.e., Vespertiliavus kasserinensis Ravel, n. sp., ?Vespertiliavus aenigma Ravel, n. sp., et Pseudovespertiliavus parva Ravel n. gen., n. sp.). Cette analyse cladistique a pour objectif d'évaluer la position de ces nouveaux taxons au sein de la super-famille des Emballonuroïdea (Emballonuridae + Nycteridae). Pour ce faire, le choix s'est porté sur 65 caractères morphologiques dont 6 mandibulaires (caractères 1 à 6) et 59 en relation avec la morphologie dentaire (Annexe 1). Certains de ces caractères sont inspirés de la littérature (par ex. : Hand 1998a; Simmons \& Geisler 1998; Hand \& Kirsch 2003; Morgan \& Czaplewski 2003; Fracasso et al. 2011; Czaplewski \& Morgan 2012; Ravel et al. 2014). Les caractères sont de poids égaux, et aucune suite additive de transformation morphologique n'a été définie sur les caractères multi-états (i.e., caractères non ordonnés).

\section{Sélection des taxons}

Le groupe interne regroupe des taxons fossiles et actuels appartenant à l'ensemble Emballonuroïdea. Les taxons fossiles incluent prioritairement les nouvelles espèces décrites dans les localités de Chambi et du Glib Zegdou, lesquelles présentent a priori des affinités morphologiques avec les Emballonuridae et/ou les Nycteridae, à savoir: Khoufechia gunnelli Ravel n. gen., n. sp., Vespertiliavus kasserinensis Ravel, n. sp., ?Vespertiliavus aenigma Ravel, n. sp., et Pseudovespertiliavus parva Ravel n. gen., n. sp. Parmi les chiroptères de Chambi, Chambinycteris pusilli Ravel n. gen., n. sp. est également inclus dans l'analyse afin d'évaluer sont degré d'affinité avec l'une des deux familles. Deux des taxons présents à Chambi ont révélé de fortes similitudes morphologiques avec l'ensemble Vespertiliavus Schlosser, 1887 documenté dans le Paléogène du Quercy (par ex. : Barghoorn 1977; Legendre 1980; Remy et al. 1987; Sigé 1990; Sudre et al. 1990; Maitre 2014). Trois des espèces les mieux documentées ont été incorporées dans l'analyse: Vespertiliavus schlosseri, $V$. wingei, et $V$. gracilis. Tachypteron franzeni de Messel (MP11) a également été inclus dans la matrice dans la mesure où il représente l'un des plus anciens jalons du groupe emballonuride. Les Emballonuridae sont également représentés par le genre Dhofarella de l'Éocène terminal du Fayum (D. sigei) et de l'Oligocène supérieur de Taqah $(D$. thaleri). Le matériel associé à ce genre est très fragmentaire et se compose d'une part d'un seul fragment de mandibule portant les trois molaires pour l'espèce $D$. sigei, et d'autre part de plusieurs dents isolées pour l'espèce $D$. thaleri. Seule l'espèce $D$. thaleri a été incluse dans l'analyse car elle permet de documenter à la fois la morphologie dentaire inférieure et supérieure du genre. Chibanycteris herberti représente un Nycteridae fossile découvert dans l'Oligocène inférieur de Taqah (Sigé et al. 1994). Ce taxon n'est documenté que par quelques dents isolées qui permettent de coder des caractères sur les dentures inférieure et supérieure. Parmi les Emballonuridae actuels, les genres Emballonura Temminck, 1838 (E. atrata) et Taphozous Geoffroy, 1818 (T. melanopogon Temminck, 1841) ont été sélectionnés car ils illustrent les deux morphotypes qui caractérisent au mieux les Emballonuridae (Emballonurinae et Taphozoinae). Les Nycteridae fossiles découverts dans le Quercy n'ont pas été inclus dans l'analyse car ces derniers ne sont pas encore formellement décrits (Sigé 2011b). Le codage a donc été effectué sur deux espèces actuelles: $N$. grandis Peters, 1865 et $N$. gambiensis Andersen, 1912. Pour enraciner l'arbre et permettre une polarisation a posteriori des caractères selon la méthode de Watrous \& Wheeler (1981), un groupe externe a été considéré. Il est constitué de trois taxons primitifs découvert dans l'Éocène européen : Icaronycteris menui et Archaeonycteris brailloni, tout deux de l'Éocène inférieur du Bassin de Paris (Mutigny et Avenay, MP8+9), et Palaeochiropteryx tupaiodon de l'Éocène moyen de Messel (MP11).

\section{Analyse de parcimonie}

Étant donné le faible nombre de taxon (18), l'analyse a été effectuée en utilisant l'algorithme de recherche «Branch and Bound» (Annexe 2). La méthode et les logiciels utilisés sont expliqués dans la partie Matériel et méthodes.

\section{RÉSULTATS}

La recherche en «Branch and Bound" a fourni trois arbres également parcimonieux de 132 pas chacun, avec un indice de consistance (I.C.) de 0,576 , et un indice de rétention (I.R.) de 0,743. Un arbre de consensus strict est illustré en Figure 32. Les différences entre les topologies se situent exclusivement au niveau de la position de Chibanycteris herberti au sein des Nycteridae. Dans les trois topologies équiprobables, les Nycteridae et les Emballonuridae forment deux groupes naturels distincts.

Le clade Nycteridae comprend Nycteris gambiensis, $N$. grandis, Chibanycteris herberti, et Khoufechia gunnelli Ravel n. gen., n. sp. Ce groupe monophylétique (indice de Bremer de 4) se définit par cinq synapomorphies exclusives: les cuspides du trigonide sont très peu élevées et ne sont pas bien individualisées $\left(19^{1}\right.$; I.C. $=1$; I.R. = 1); la distance entre le paraconide et le métaconide est nettement supérieure à la distance entre le métaconide et l'entoconide sur $\mathrm{m} 2\left(23^{1}\right.$; I.C. = 1 ; I.R. = 1); l'entoconide est très réduit voir absent $\left(26^{1}\right.$; I.C. $=1$; I.R. = 1); l'aplanissement distal du talon des M1-2 est très prononcé $\left(58^{0}\right.$; I.C. $=1$; I.R. $\left.=1\right)$; la M3 est très réduite en taille $\left(62^{1}\right.$; I.C. $=1 ;$ I.R. = 1). Certaines de ces synapomorphies ne peuvent être vérifiées pour Chibanycteris herberti à cause du manque de matériel lui étant associé. Au sein des Nycteridae, Khoufechia gunnelli Ravel n. gen., n. sp. se positionne à la base des trois autres taxons. En raison des données fragmentaires relatives à $C$. herberti, la position de ce taxon est très instable. Cette forme de Taqah est parfois espèce sœur de Nycteris gambiensis, parfois espèce sœur de $N$. grandis, ou alors se situe à la base du clade réunissant les deux espèces actuelles (Emballonura atrata et Taphozous melanopogon). Toutefois, ces trois taxons partagent un protocône très réduit qui constitue ainsi une 
synapomorphie exclusive et non ambigüe du clade $\left(51^{1}\right.$; I.C. $=$ 1 ; I.R. = 1). L'instabilité des positions de certains nyctéridés ne permet pas ici de résoudre les relations phylogénétiques intrafamiliales, notamment en ce qui concerne les relations entre les formes les plus dérivées. Si l'on supprime de la matrice le taxon fossile de Taqah ( $C$. herberti), aucun changement n'apparaît dans la topologie à l'exception d'une meilleure résolution du clade Nycteris. Ce regroupement d'espèces se caractérise par une synapomorphie exclusive et non ambigüe: la présence d'une unique prémolaire supérieure $\left(36^{2} ;\right.$ I.C. = $1 ;$ I.R. = 1). Tous ces taxons partagent également la largeur du trigonide supérieure à celle du talonide sur m1-2 (251; I.C. =0,66; I.R. =0,66). Cette caractéristique représente toutefois une convergence avec Palaeochiropteryx tupaiodon.

Les Emballonuridae regroupent l'ensemble des espèces Vespertiliavus européennes et africaines, ainsi que Tachypteron franzeni, Dhofarella thaleri et les deux espèces actuelles, Emballonura atrata et Taphozous melanopogon. Chambinycteris pusilli Ravel n. gen., n. sp. se positionne à la base (premier rameau) du clade Emballonuridae. Ce clade est soutenu par trois synapomorphies exclusives et non ambigües (I.C. $=1$; I.R. = 1): la position distale de l'hypoconulide par rapport à l'entoconide et de leur proximité (292); la dissymétrie très prononcée des " $\mathrm{V}$ » antérieurs et postérieurs de l'ectolophe sur M1 et M2 (411); la forte inclinaison du bord buccal de M1 $\left(42^{0}\right)$. Si le clade des Emballonuridae est bien défini, le groupe se décompose en une succession d'embranchements. Cet agencement ne permet pas de mettre en évidence des regroupements intrafamiliaux. Il est cependant possible de noter une certaine cohérence dans la distribution des caractères au sein des Emballonuridae. Certains regroupements se font donc par le soutien de synapomorphies non homoplasiques et non ambigües (I.C. $=1$; I.R. = 1). Le genre Tachypteron se positionne proche de la base du clade juste après l'espèce C. pusilli Ravel n. gen., n. sp. Tachypteron partage avec les autres Emballonuridae (excepté Chambinycteris Ravel, n. gen.) une protofosse étendue selon l'axe mésiodistal $\left(54^{\circ}\right)$ et un faible aplanissement du talon (581). Ce dernier est plus variable chez Pseudovespertiliavus Ravel, n. gen. Le genre Pseudovespertiliavus Ravel, n. gen. se place entre Tachypteron et l'ensemble Vespertiliavus. Pseudovespertiliavus Ravel, n. gen., Vespertiliavus, Dhofarella et les deux genres actuels forment un groupe monophylétique supporté par une synapomorphie exclusive et non ambigüe (I.C. = 1 ; I.R. = 1). Ces taxons présentent une ouverture distale de la protofosse $\left(55^{1}\right)$. L'ensemble Vespertiliavus apparaît totalement paraphylétique et constitue une succession d'embranchements entre Tachypteron franzeni et Emballonura atrata. Les espèces des genres Vespertiliavus, Dhofarella, Emballonura et Taphozous partagent néanmoins un entoconide élevé et incliné distalement, ce qui constitue une synapomorphie exclusive $\left(26^{0} ;\right.$ I.C. $=1$; I.R. $\left.=1\right)$. Un groupe formé des espèces ? Vespertiliavus aenigma Ravel, n. sp., $V$. gracilis, V. kasserinensis Ravel, n. sp., Emballonura atrata, Dhofarella thaleri et Taphozous melanopogon est soutenu par la connexion entre une protofosse basse et le bassin du talon $\left(56^{0}\right.$; I.C. $=1 ;$ I.R. = 1). Enfin, Vespertiliavus gracilis et $V$. kasserinensis Ravel, n. sp. sont regroupés avec $E$. atrata,
$D$. thaleri et T. melanopogon par la morphologie sinueuse de l'entocristide en vue occlusale $\left(27^{1}\right.$; I.C. $=1$; I.R. $\left.=1\right)$. Au niveau du groupe couronne, le fossile Dhofarella se positionne préférentiellement avec Taphozous, ce clade étant groupe-frère du genre Emballonura.

KHOUFECHIA GUNNELLI RAVEL N. GEN., N. SP., TAXON BASAL DU CLADE NYCTERIDAE

La description originale (Sigé 1991b) de l'unique spécimen attribué à un Rhinolophoidea indét. (que nous référons ici à Khoufechia gunnelli Ravel n. gen., n. sp.), était basée sur un fragment de molaire supérieure (Sigé 1991b). Sur ce fragment buccal de dent, la morphologie de l'ectolophe, formant un «W» ouvert et presque symétrique, les faibles proportions de la protofosse, du protocône et des postprotocrêtes, et la présence d'un talon projeté distolingualement, non apparent mais nettement indiqué, ont conduit l'auteur à rapprocher cette forme de Chambi du grand ensemble Rhinolophoidea. Ce rapprochement fut remis en question par la comparaison du spécimen avec Chibanycteris herberti découvert dans l'Oligocène de Taqah (Sigé et al. 1994). L'auteur émit alors l'hypothèse que le spécimen de Chambi pourrait en fait représenter une espèce de Nycteridae très ancienne. L'apport ici d'un matériel plus complet a permis de mieux cerner la morphologie dentaire de ce taxon et de définir Khoufechia gunnelli Ravel n. gen., n. sp. D’un point de vue phylogénétique, nos résultats soutiennent la position de $K$. gunnelli Ravel n. gen., n. sp. à la base des Nycteridae (Fig. 32). Les Nycteridae sont caractérisés par un entoconide très réduit et positionné près de la base du métaconide. Chez Khoufechia Ravel, n. gen., l'entoconide est légèrement plus développé et plus distal sur les m1-2s. Ces états de caractères constituent un stade plus primitif au sein des Nycteridae.

\section{PSEUDOVESPERTILIAVUS PARVA RAVEL N. GEN., N. SP., UN EMBALLONURIDAE PRIMITIF}

La description et la comparaison de Pseudovespertiliavus parva Ravel n. gen., n. sp. ont permis de déceler une morphologie dentaire combinant des caractères que l'on retrouve au sein des deux groupes frères Emballonuridae et Nycteridae. Les résultats de l'analyse cladistique attestent la position de $P$. parva Ravel n. gen., n. sp. au sein des Emballonuridae (Fig. 32) par la présence d'apomorphies partagées avec les autres taxons constituant cet ensemble: la position de l'hypoconulide, distale et très proche de l'entoconide $\left(29^{2}\right.$; I.C. $=1$; I.R. $\left.=1\right)$; la dissymétrie très prononcée des « $\mathrm{V}$ » antérieur et postérieur de l'ectolophe sur M1 et M2 (411 ; I.C. = 1; I.R. = 1); la forte inclinaison du bord buccal de M1 (420; I.C. = 1 ; I.R. = 1). Les caractères partagés avec les Nycteridae, mais non exclusifs au groupe (i.e., homoplasie structurée) ont contribué à rapprocher le taxon de Chambi de la base du clade Emballonuridae. Ils concernent notamment la présence d'un hypoconulide sur m3 $\left(33^{0}\right.$; I.C. $=0,33$; I.R. $\left.=0,6\right)$, l'inclinaison forte de la cristide oblique $\left(28^{1} ;\right.$ I.C. $\left.=0,33 ; \mathrm{I} . \mathrm{R}=0,5\right)$, et l'épaisseur plus faible du cingulum lingual $\left(61^{0+1} ;\right.$ I.C. $=50$; I.R. $\left.=50\right)$. Pseudovespertiliavus parva Ravel n. gen., n. sp. apparaît toutefois moins basal que Tachypteron franzeni qui représente un 
taxon plus primitif, du moins par sa morphologie dentaire. En effet, Pseudovespertiliavus Ravel, n. gen. présente une ouverture nette et distale de la protofosse (551) qui constitue un état plus dérivé que chez Tachypteron, chez lequel la protofosse est fermée distalement $\left(55^{\circ}\right)$. Cette apomorphie présente chez Pseudovespertiliavus Ravel, n. gen. contribue à le rapprocher de l'ensemble Vespertiliavus et des taxons actuels.

\section{LA PARAPHYLLIE DU GENRE VESPERTILIAVUS}

Le genre Vespertiliavus fut identifié par Schlosser en 1887 à partir d'un matériel fossile provenant des karsts paléogènes du Quercy. En l'état de nos connaissances actuelles du registre fossile, le genre regroupe neuf espèces formant six lignées (Maitre 2014) qui s'étendent depuis l'Éocène moyen avec l'espèce V. lapradensis (Cuzal, MP13; Marandat et al. 1993) jusqu'à l'Oligocène supérieur avec l'espèce $V$. gerscheli (Garouillas, MP25; Sigé 1990). Considérant ces occurrences, le genre Vespertiliavus représente l'une des plus anciennes formes emballonurides avec Tachypteron franzenni, présent dès le niveau repère MP11 de Messel (Storch et al. 2002). La position de Vespertiliavus au sein des Emballonuridae a été étudiée par Barghoorn (1977). Ce dernier a réalisé une analyse phylogénétique à partir d'une vingtaine de caractères crâniens, en considérant uniquement l'espèce $V$. bourguignati pour laquelle le matériel crânien est particulièrement abondant et complet. Les résultats obtenus ont montré les affinités du genre Vespertiliavus avec les genres actuels tels que Taphozous et Emballonura, les trois formes formant un clade se branchant au sommet des Emballonuridae (Fig. 32). Les résultats de l'analyse cladistique obtenus dans ce travail ne permettent pas d'identifier des synapomorphies pouvant regrouper les différentes espèces du genre Vespertiliavus. Ceci s'explique notamment par l'importante variabilité interspécifique (mais également intraspécifique) de la morphologie dentaire du genre. Bien que paraphylétique, le groupe est bien défini entre le genre Tachypteron d'une part et le genre Emballonura (actuel) d'autre part. Les deux espèces de Chambi, ?V. aenigma Ravel, n. sp. et $V$. kasserinensis Ravel, n. sp., sont également incluses dans ce regroupement paraphylétique. Étant donné l'âge du niveau fossilifère de Chambi (incluant les loci 1, 2 \& 3; i.e., Éocène inférieur terminal-Éocène moyen basal), ces deux nouvelles espèces représentent dès lors les plus anciennes occurrences du genre Vespertiliavus. Ces deux espèces permettent également d'élargir l'aire de répartition du genre, qui était jusque là documenté uniquement en Europe. Nos résultats phylogénétiques soutiennent l'hypothèse d'une origine africaine pour les Vespertiliavus depuis un groupe souche représenté par Pseudovespertiliavus parva Ravel n. gen., n. sp. La présence du genre Vespertiliavus à Chambi et dans le Quercy illustre une dispersion transtéthysienne, entre l'Afrique du Nord et le sud de l'Europe, qui a certainement prit place durant l'Éocène moyen.

CHAMBINYCTERIS PUSILLI RAVEL N. GEN., N. SP., LA BASE DES EMBALLONURIDAE?

La description et la comparaison du matériel dentaire relatif à Chambinycteris pusilli Ravel n. gen., n. sp. n'ont pas per- mis de définir son appartenance à une famille de chiroptère moderne connue. La morphologie dentaire qui caractérise ce nouveau genre présente une mosaïque de caractères à la fois rhinolophoïdes et emballonuroïdes (et plus précisément emballonurides). Ce type de combinaison a déjà été observé au niveau de la morphologie dentaire des Mixopterigydae, mais elle n'implique pas les mêmes structures. Le fait d'inclure C. pusilli Ravel n. gen., n. sp. dans l'analyse cladistique permet d'identifier la présence d'apomorphies exclusivement partagées avec l'ensemble des Emballonuridae, appuyant ainsi un rattachement de ce taxon à cette famille. Chambinycteris pusilli Ravel n. gen., n. sp. occupe en effet une position basale à l'intérieur du clade Emballonuridae (avant Tachypteron franzeni; Fig. 32). Leurs synapomorphies sont la position distale et très proche de l'hypoconulide par rapport à l'entoconide (292), la dissymétrie très prononcée des « $\mathrm{V}$ » antérieur et postérieur de l'ectolophe sur M1 et M2 (411), et la forte inclinaison du bord buccal de M1 $\left(42^{\circ}\right)$. Il est possible que $C$. pusilli Ravel n. gen., n. sp. représente le taxon le plus primitif au sein des Emballonuridae, mais cette hypothèse reste à confirmer par un matériel plus abondant.

\section{IMPLICATIONS PALÉOBIOGÉOGRAPHIQUES}

\section{LES DÉBUTS DE L'HISTOIRE ÉVOLUTIVE DES HiPPOSIDERIDAE}

Les Hipposideridae actuels incluent neuf genres comprenant au total 65 espèces qui se répartissent exclusivement sur l'Ancien Monde (par ex. : Koopman 1994 ; Simmons \& Conway 2003). Cette famille a longtemps été considérée comme un sous-ensemble des Rhinolophidae, partageant de nombreuses ressemblances anatomiques (par ex. : Miller 1907; Simmons \& Geisler 1998). D’un point de vue morphologique, les Hipposideridae se distinguent des Rhinolophidae par des ceintures pectorales et pelviennes qui sont plus différenciées (fusion des première et seconde côtes, ainsi que des vertèbres lombaires associées, connexion du processus aciculaire avec l'avant de l'ilium formant un large foramen pré-acétabulaire), par la présence de deux phalanges à chaque doigt, et par des vertèbres lombaires fusionnées en une tige solide (Miller 1907). De plus, l'articulation distale de l'humérus des Hipposideridae se différencie de celle des Rhinolophidae par la projection médiale moins prononcée de la trochlée, ainsi qu'une épitrochlée un peu moins développée portant un processus plus réduit (Sigé 1990). Au niveau de la morphologie dentaire, les différences sont minces entre les deux familles. Seule l'absence d'une p3 vestigiale permet de distinguer nettement les Hipposideridae. Le reste des divergences concerne uniquement certains genres hipposidérides, comme par exemple les genres Hipposideros, Asellia Gray, 1838 et Anthops Thomas, 1888 qui possèdent une M3 et une $\mathrm{m} 3$ très réduite comparées à celles des Rhinolophidae. Les récentes investigations phylogénétiques moléculaires et morphologiques appuient de façon robuste la validité du clade Rhinolophidae + Hipposideridae. Certaines études ont estimé l'âge de divergence entre ces deux familles au début de l'Éocène supérieur, 


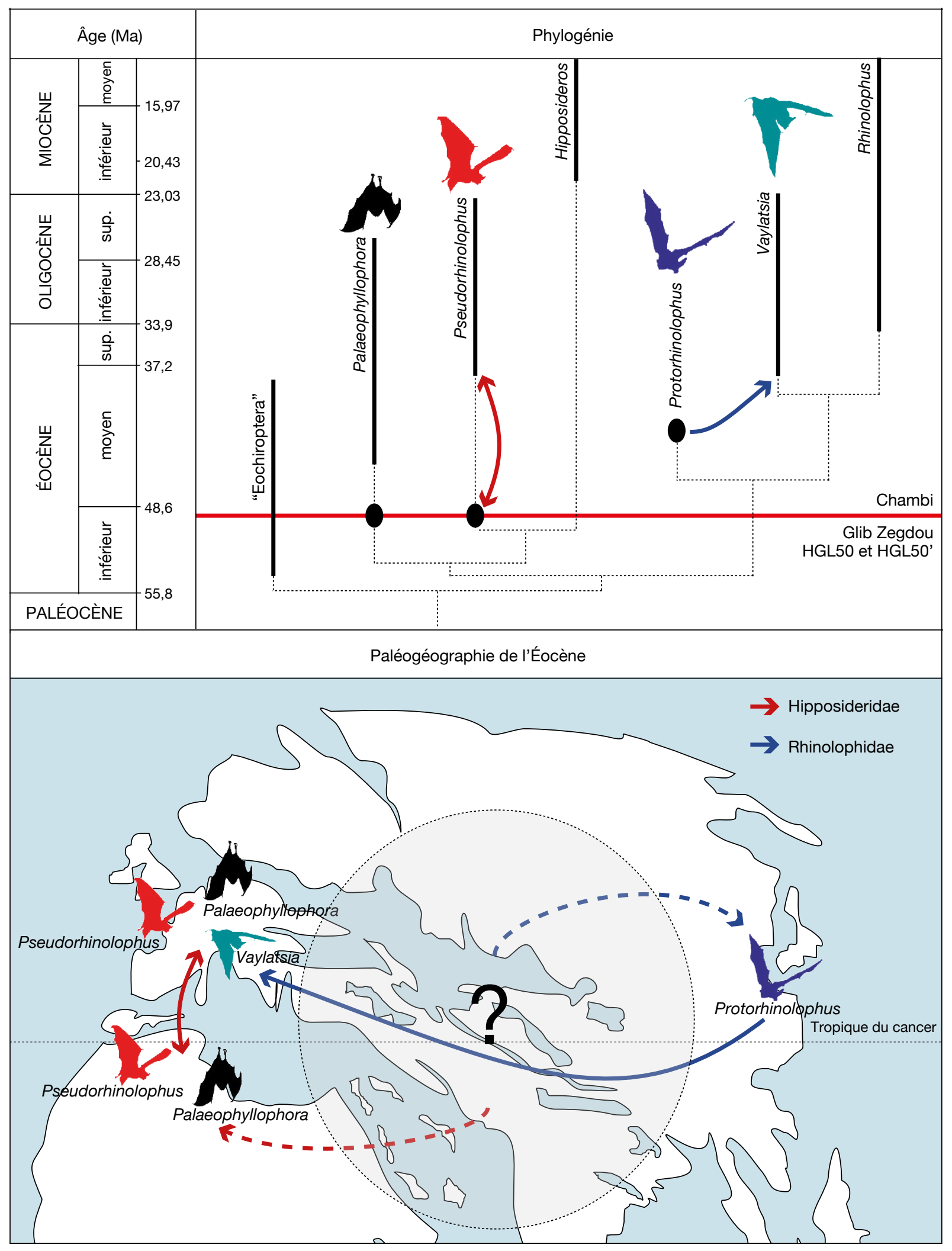

FIG. 33. - Hypothèse paléo-phylogéographique sur les premières phases de dispersion des Rhinolophidae et Hipposideridae. L'échelle des temps géologiques est positionnée en haut à gauche. L'hypothèse phylogénétique est reprise de l'arbre obtenu après l'analyse cladistique en «Branch and Bound " (Fig. 31). Les événements de dispersion durant l'Éocène sont figurés à droite. Les flèches en trait continu illustrent les mouvements de dispersion les plus probables supportés par les données fossiles. Les flèches en trait pointillé représentent les possibles événements de dispersion antérieurs, n'étant soutenus par aucune donnée paléontologique. Les flèches en bleu représentent les mouvements de dispersion concernant les Rhinolophidae uniquement. Les flèches en rouge représentent les mouvements de dispersion concernant les Hipposideridae uniquement. 
soit autour de $37 \mathrm{Ma}$, depuis un groupe souche asiatique (Teeling et al. 2005; Agnarsson et al. 2011). Les localités de Chambi révèlent deux genres d' Hipposideridae qui étaient déjà connus dans des niveaux plus récents (milieu de l'Éocène moyen) du Quercy en France. Cette découverte recule considérablement l'âge de divergence des Hipposideridae, estimé jusqu'à présent à l'Éocène moyen par les données paléontologiques et moléculaires (Teeling et al. 2005; Lack et al. 2010; Agnarsson et al. 2011). La discussion à propos des deux genres représentés à Chambi met en évidence un échange faunique entre l'Afrique du Nord et l'Europe qui impliquerait l'ensemble des Hipposideridae (Fig. 33). Paradoxalement, les Rhinolophidae ne sont pas documentés à Chambi malgré la grande diversité des chiroptères dans cette localité. La découverte de Protorhinolophus shanghuangensis daté du milieu de l'Éocène moyen à Shanghuang en Chine, a permis de mettre en évidence l'existence d'une éventuelle voie migratoire entre l'Asie de l'Est et l'Europe qui n'impliquait que les Rhinolophidae (Ravel et al. 2014). Cet événement de dispersion serait effectif à l'Éocène moyen compte tenu de la présence plus tardive des Rhinolophidae dans le Quercy par rapport aux Hipposideridae. Une origine commune pour les Hipposideridae et les Rhinolophidae en Asie centrale constitue ainsi un scénario plus parcimonieux. Cependant, l'absence totale de chiroptère fossile paléogène dans cette province ne permet pas de confirmer ou d'infirmer cette hypothèse.

\section{IMPLICATION SUR L'ORIGINE DES EMBALLONUROIDEA}

La super-famille Emballonuroidea forme un groupe naturel incluant les Emballonuridae et les Nycteridae. Ce regroupement est très bien soutenu par les études phylogénétiques basées sur des données morphologiques et moléculaires (par ex. : Eick et al. 2005; Simmons 2005a, b; Teeling 2009; Simmons et al. 2010 ; Teeling et al. 2012). Les données paléontologiques soutiennent également ce clade, notamment par la découverte d'une forme fossile dans l'Oligocène supérieur du Quercy appartenant aux Nycteridae, mais présentant également des affinités morphologiques avec les Emballonuridae (Sigé 2011b). Les nouveaux taxons fossiles de Chambi et du Glib Zegdou apportent des arguments supplémentaires en faveur du regroupement des deux familles. Ceci est particulièrement soutenu par la morphologie dentaire de Pseudovespertiliavus Ravel, n. gen. caractérisée par une combinaison de caractères connus pour certains chez les Emballonuridae, et pour d'autres chez les Nycteridae. Les deux familles sont représentées dès l'Éocène inférieur terminal - Éocène moyen basal par des taxons très proches de la divergence Emballonuridae/Nycteridae. Compte tenu de l'ancienneté et du degré évolutif des Nycteridae et des Emballonuridae de Chambi et du Glib Zegdou, une origine commune des deux familles depuis une souche africaine est fortement suggérée ici. Cependant la nature et les modes de dispersion qui ont suivi cette radiation africaine semblent avoir été très différents d'une famille à l'autre. Ces aspects seront discutés dans les paragraphes suivants, pour chaque famille.
UNE HISTOIRE ÉVOLUTIVE DES NYCTERIDAE

ÉTROITEMENT LIÉE À L'AFRIQUE

Le registre fossile de la famille des Nycteridae est particulièrement pauvre. L'essentiel des fossiles documente des espèces du genre Nycteris du Plio-Pléistocène d'Afrique centrale (Black \& Krishtalka 1986; Pocock 1987). Une forme nyctéride est également signalée dans le Miocène inférieur d'Afrique orientale (Butler 1984). Le matériel référé est cependant trop fragmentaire pour en effectuer une étude approfondie. Plusieurs dents isolées provenant de l'Oligocène supérieur (MP29) de Verneuil (Limagne bourbonnaise, France) pourraient appartenir à un Nycteridae (Hugueney 1984), mais le matériel est trop fragmentaire pour confirmer l'attribution à cette famille. Jusqu'à présent, Chibanycteris herberti, découvert dans l'Oligocène inférieur de Taqah, était le seul Nycteridae paléogène bien identifié (Sigé et al. 1994). C'est en comparant le taxon d'Oman avec le spécimen découvert à Chambi que Sigé et al. (1994) ont émis l'hypothèse d'une probable affiliation entre les deux taxons. Dès lors, ces auteurs ont suggéré l'hypothèse d'une origine africaine pour les Nycteridae durant l'Éocène inférieur terminal. L'analyse de la morphologie dentaire de Khoufechia gunnelli Ravel n. gen., n. sp. vient appuyer cette hypothèse du fait de la confirmation du statut familial du taxon tunisien (i.e., Nycteridae) et de sa position basale au sein de cette famille. De plus, la répartition actuelle des Nycteridae semble être corrélée avec une histoire fortement ancrée en Afrique. En effet, dans la nature actuelle, le genre Nycteris comprend 14 espèces au total dont la grande majorité (i.e., 11 sur 14 espèces) se retrouve en Afrique et quelques unes (i.e., 3 sur 14 espèces) se retrouvent à Madagascar, en Indonésie, en Thaïlande et en Malaisie (Miller 1907 ; Van Cakenberghe \& De Vree 1985, 1993a, b, 1998; Simmons \& Conway 2003). Si l'évolution des Nycteridae est étroitement liée au continent africain depuis l'Éocène inférieur, un épisode de dispersion depuis l'Afrique vers l'Europe durant l'Oligocène supérieur a été révélé par la présence des Nycteridae fossiles dans le Quercy (Mas de Pauffié [MP26] et La Devèze [MP26]; Sigé 2011b). Cette occurrence vient s'ajouter au possible Nycteridae plus récent de Verneuil. L'arrivée des Nycteridae en Europe durant l'Oligocène supérieur a probablement précédé la vague de dispersion plus récente vers l'Asie conduisant à la présence des quelques espèces en Asie du Sud-Est (Van Cakenberghe \& De Vree 1985, 1993a, b, 1998).

LA DISPERSION DES EMBALLONURIDAE DURANT L'ÉOCÈNE Les Emballonuridae sont bien diversifiés dans le Paléogène européen. C'est également la seule famille moderne présente dans la localité de Messel, avec Tachypteron franzenni (Storch et al. 2002), ce qui en fait l'une des plus anciennes familles actuelles représentées dans le registre fossile. Mais c'est avant tout dans l'Éocène moyen du Quercy que les Emballonuridae montrent une grande diversité avec le genre Vespertiliavus (par ex. : Barghoorn 1977; Legendre 1980; Remy et al. 1987; Sigé 1990; Sudre et al. 1990; Maitre 2014). Dans le Paléogène d'Afrique et de la Péninsule Arabique, le 


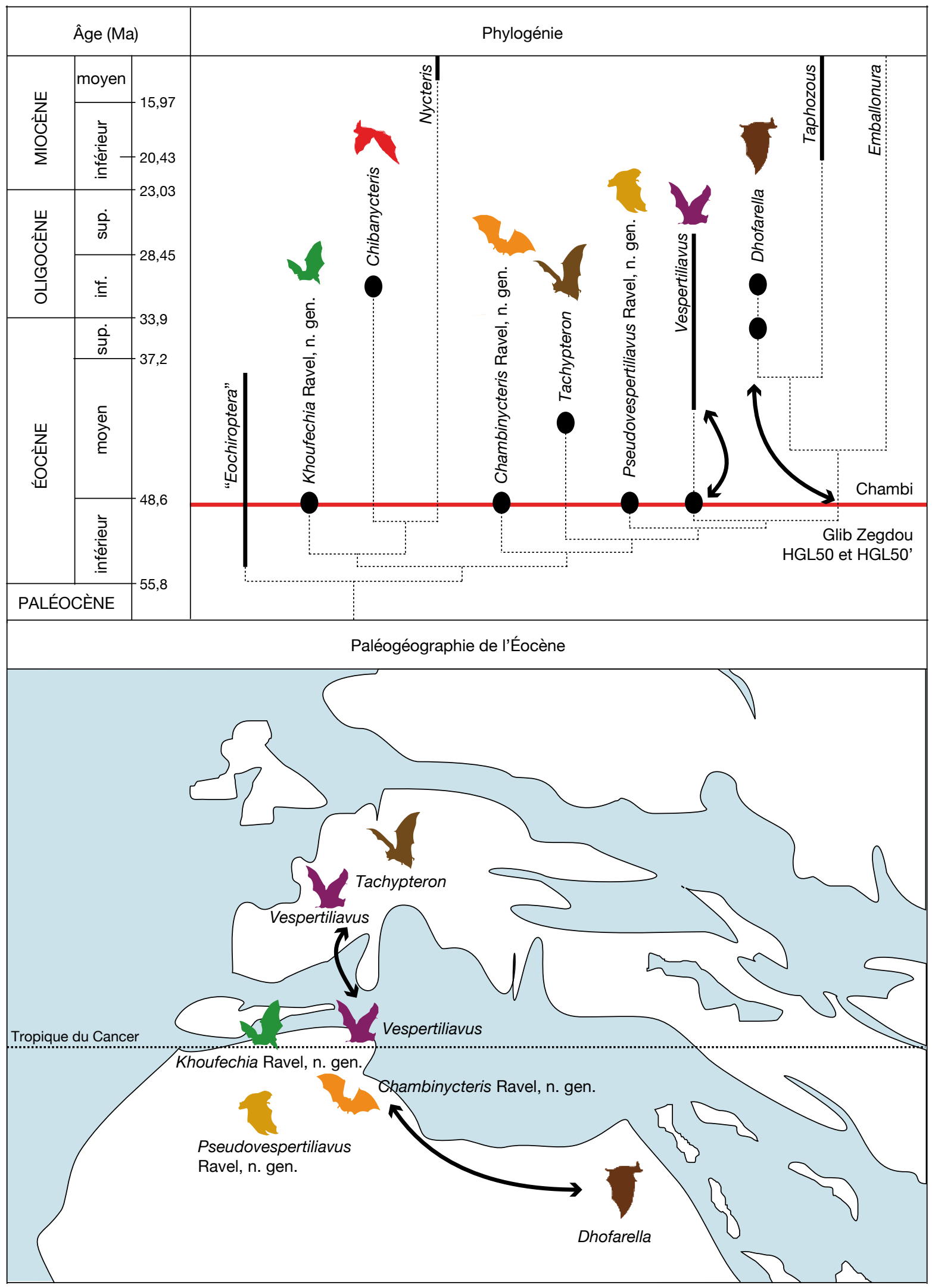

FIG. 34. - Hypothèse paléo-phylogéographique sur les premières phases de dispersions des Emballonuridae et des Nycteridae. L'échelle des temps géologiques est positionnée en haut à gauche. L'hypothèse phylogénétique est reprise de l'arbre de consensus strict (Fig. 32). Les événements de dispersion durant l'Éocène sont figurés à droite. Les flèches illustrent les mouvements de dispersion impliquant les Emballonuridae. 
groupe est documenté de manière plus sporadique avec le genre Dhofarella, présent à l'Éocène supérieur et l'Oligocène inférieur (Sigé et al. 1994; Gunnell et al. 2008). La faune de Chambi décrite ici comprend une diversité spécifique non négligeable comprenant trois à quatre espèces (si Chambinycteris Ravel, n. gen. est confirmé). Cette diversité implique autant de formes primitives (i.e., Chambinycteris pusilli Ravel n. gen., n. sp. et Pseudovespertiliavus parva Ravel n. gen., n. sp.) que de morphologies plus dérivées et proches des formes du Quercy (i.e., ?Vespertiliavus aenigma Ravel, n. sp. et Vespertiliavus kasserinensis Ravel, n. sp.). Les affinités avec la faune du Quercy suggèrent l'existence d'une dispersion transtéthysienne pour les Emballonuridae, de toute évidence durant l'Éocène moyen (Fig. 34), un événement qui avait déjà été envisagé par Sigé et al. (1994). Ce scénario reste plausible mais semble ne concerner que l'ensemble Vespertiliavus. Les événements de dispersion sont moins évidents avec les formes plus primitives telles que Tachypteron, Pseudovespertiliavus Ravel, n. gen. et Chambinycteris Ravel, n. gen. En effet, les espèces qui présentent une morphologie conservatrice sont représentées en Afrique du Nord et en Europe, mais les relations phylogénétiques entre elles restent incertaines. Le registre fossile relatif aux Emballonuridae est pour le moment trop incomplet pour pouvoir préciser le sens et la fréquence des mouvements de dispersion des premiers Emballonuridae.

\section{TAPHONOMIE DES GISEMENTS DE CHAMBI ET DU GLIB ZEGDOU}

La faune de Chambi est composée de neuf espèces appartenant à cinq familles modernes. Une telle concentration est surprenante pour un gisement constitué de dépôts de type calcaire lacustre. La taphonomie, pour les chiroptères, est un élément important à prendre en compte afin de discuter de la paléoécologie, en particulier du type d'habitat (Kowalski 1995). Dans le registre fossile, les chiroptères primitifs (i.e., «Eochiroptera») sont presque exclusivement issus de dépôts fluvio-lacustres contribuant parfois à la bonne préservation de leur squelette comme à Messel ou au Wyoming. Au contraire, les formes modernes semblent plus souvent associées aux remplissages karstiques (Sigé \& Legendre 1983). Les gisements du Quercy ou de Shanghuang (Ravel et al. 2014) en sont un parfait exemple. Ce type de dépôt, souvent associé à un matériel abondant et bien préservé, est étroitement lié à un habitat troglodyte. Ce dernier est largement reconnu chez les microchiroptères. Sans s'y opposer, la sédimentologie caractérisant les localités de Chambi et du Glib Zegdou ne permet pas de confirmer le comportement cavernicole des chiroptères. Cependant, plusieurs faits notables soutiennent ce type de comportement. Plusieurs espèces de Chambi ont montré des affinités avec des formes du Quercy qui, comme l'indique leur contexte géologique, habitaient d'anciennes grottes. Les petites espèces étaient possiblement capables de repérer et de chasser de petites proies grâce à une écholocation sophistiquée. Cette capacité devait également leur permettre une grande manœuvrabilité dans des espaces clos avec une faible visibilité. La présence de chiroptères cavernicoles dans des sédiments fluvio-lacustres peut s'expliquer par l'accumulation de pelotes de régurgitation rejetées par les oiseaux de proies, principaux prédateurs des chauvessouris (Kowalski 1995). La diversité des chiroptères relevée à Chambi paraît toutefois trop importante pour pouvoir être la conséquence d'une accumulation de pelotes de régurgitation. De plus, l'émail des dents ne possèdent pas de traces de digestion organique qui permettrait de fait de déceler une prédation sur les petits mammifères (par ex. : Dodson \& Wexlar 1979; Laudet \& Selva 2005). Un autre facteur pouvant intervenir dans l'agrégation de fossiles de chiroptères est le transport par l'eau (Kowalski 1995). Cette dernière hypothèse paraît plus probable. L'amoncellement des fossiles retrouvé à Chambi et au Glib Zegdou pourrait être alors la conséquence du lessivage d'un réseau ancien de grottes par une montée des eaux.

\section{CONCLUSION}

Une étude systématique de l'ensemble du matériel dentaire provenant de Chambi et du Glib Zegdou a permis de mettre en évidence la présence en Afrique du Nord d'une surprenante diversité de chiroptères modernes dès la fin de l'Éocène inférieur. À Chambi notamment, en plus des Philisidae déjà décrits (Vespertilionoidea; Sigé 1991b; Ravel et al. 2012), la faune de chiroptères comprend les plus anciens représentants de nombreuses familles actuelles: des Hipposideridae (i.e., ?Palaeophyllophora tunisiensis Ravel, n. sp. et Hipposideros [Pseudorhinolophus] africanum Ravel, n. sp.), des Emballonuridae (i.e., ?Vespertiliavus aenigma Ravel, n. sp., Vespertiliavus kasserinensis Ravel, n. sp., Pseudovespertiliavus parva Ravel n. gen., n. sp., et possiblement Chambinycteris pusilli Ravel n. gen., n. sp.), un Nycteridae (Khoufechia gunnelli Ravel n. gen., n. sp.), et un Vespertilionidae indéterminé. Un Necromantidae (?Necromantis fragmentum Ravel, n. sp.) est également identifié à Chambi par une espèce qui possède de nombreuses affinités avec le genre européen Necromantis. Une dernière espèce est reconnue au Glib Zegdou (Drakonycteris glibzedouensis Ravel n. gen., n. sp.). Cependant, ce taxon possède une morphologie très originale qui ne permet pas de le rapprocher d'une famille connue. Du fait de la singularité morphologique de ce taxon, cette espèce pourrait éventuellement représenter une famille éteinte encore inconnue qui, comme les Philisidae, aurait une histoire profondément et essentiellement liée à l'Afrique. La plupart des autres genres identifiés à Chambi (Vespertiliavus, Necromantis, Hipposideros [Pseudorhinolophus] et Paleophyllophora) montrent une composition taxonomique qui rappelle les faunes plus récentes du Quercy. Ces affinités sont de toute évidence le résultat d'échanges fauniques entre l'Afrique du Nord et le sud de l'Europe au cours de l'Éocène. Ces échanges ont impliqué les Hipposideridae, les Emballonuridae et les Necromantidae. En revanche, les Nycteridae et les Philisidae, et plus globalement les Vesper- 
tilionoidea, ont vraisemblablement eu une aire de répartition plus limitée, pour le moment exclusivement restreinte à l'Afrique durant l'Éocène inférieur et moyen.

Ces événements de dispersion soulignent les capacités de migration des chiroptères au cours du Paléogène - à la faveur de leur locomotion spécialisée au vol, certainement déjà bien développé dès le début de l'Éocène, et à l'origine de leur dispersion rapide sur de grandes distances. De nos jours, certaines chauves-souris peuvent parcourir plus de $3000 \mathrm{~km}$ en une saison (Fleming \& Eby 2003). Le déplacement aérien des chiroptères présente un avantage lorsqu'il s'agit d'explorer des nouvelles niches difficilement accessibles par voie terrestre (par ex. : de bas reliefs ou des étendues d'eau). Cette mobilité a du permettre des flux migratoires beaucoup plus complexes et plus fréquents qu'il n'y parait, comprenant notamment des migrations saisonnières qui ne peuvent pas être détectées avec le registre fossile actuel.

La taphonomie est généralement un bon indicateur pour renseigner le type d'habitat des chiroptères. Les formes modernes cavernicoles sont souvent associées à des remplissages karstiques. La présence de faunes de chiroptères modernes dans des dépôts fluvio-lacustres tels ceux de Chambi et du Glib Zegdou, est en fait singulière. L'accumulation d'une telle richesse spécifique, notamment à Chambi, suggère un transport hydrologique depuis un réseau ancien de grottes qui devait abriter de nombreuses colonies de chiroptères cavernicoles.

\section{Remerciements}

Les auteurs remercient Mr. le Vice-Chancelier de l'Université de Tlemcen (Algérie) et les autorités des régions de Béchar et Tindouf pour leur assistance lors des chantiers de fouilles dans les Gour Lazib, Algérie. Merci à Pierre-Henri Fabre (ISE-M), Xavier Valentin (Institut de Paléoprimatologie et Paléontologie humaine: Évolution et Paléoenvironnements [IPHEP]), Vincent Lazzari (IPHEP), Lionel Hautier (ISE-M), Helder Gomes Rodrigues (ISE-M) et Emmanuel Fara (Université de Dijon) pour leur aide sur les chantiers des Gour Lazib. Nous addressons nos remerciements à Suzanne Jiquel (ISE-M), Anusha Ramdardshan (ISE-M), Bernard Marandat (ISE-M) et Faouzi M'Nasri (ONM, Tunis) pour leur participation à l'expédition dans la région de Kasserine, Tunisie. Nous sommes reconnaissants envers Mustapha Ben Haj Ali, ancien chef du Service géologique de l'ONM (Tunis) pour sa collaboration enthousiaste au projet d'études paléontologiques en Tunisie. Nous addressons nos sincères remerciement à Bernard Sigé pour ses remarques, ses conseils avisés et nos discussions basées sur sa profonde connaissance des chiroptères fossiles. Un grand merci à Maeva Orliac (ISE-M) pour son aide et ses conseils sur les analyses cladistiques. Enfin, nous remercions Chantal Cazevieille (Centre de Ressources en Imagerie cellulaire, Montpellier) pour l'accès au Microscope électronique à Balayage. Ce travail a été financé par le Programme français ANR-ERC PALASIAFRICA (ANR-08-JCJC-0017), une subvention du Conseil scientifique (CS) de l'Université de Montpellier (UM), et par l'ONM de Tunis (AC-1785). Ceci est la publication ISEM 2016-022 SUD.

\section{RÉFÉRENCES}

Adaci M., Tabuce R., Mebrouk, F., Bensalah M., Fabre P.-H., Hautier L., Jaeger J.-J., Lazzari V., Mahboubi M., MarivauX L., Otero O., Peigné S. \& Tong H. 2007. — Nouveaux sites à vertébrés paléogènes dans la région des Gour Lazib (Sahara nord-occidental, Algérie). Comptes Rendus Palevol 6: 535-544. http://dx.doi.org/10.1016/j.crpv.2007.09.001

AgnarsSON I., Zambrana-Torrelio C. M., Flores-Saldana N. P. \& MaY-Collado L. J. 2011. — A time calibrated species-level phylogeny of bats (Chiroptera, Mammalia). PLOS Currents Tree of Life, 2011 Feb 4, Edition 1. http://dx.doi.org/10.1371/ currents.RRN1212

BARGHOORN S. F. 1977. — New material of Vespertiliavus Schlosser (Mammalia, Chiroptera) and suggested relationships of emballonurid bats based on cranial morphology. American Museum Novitates 2618: 1-29.

Beard K. C., Sigé B. \& Krishtalka L. 1992. — A primitive vespertilionoid bat from the Early Eocene of Central Wyoming. Compte rendu de l'Académie des Sciences de Paris 314: 735-741.

Black C. C. \& KrishtalKa L. 1986. — Rodents, bats, and insectivores from the Plio-Pleistocene sediments to the East of Lake Turkana, Kenya. Contributions in Science, Natural History Museum of Los Angeles Co 372: 1-15.

BREMER K. 1994. - Branch support and tree stability. Cladistic 10: 295-304

BuTler P. M. 1984. - Macroscelidae, Insectivora and Chiroptera from the Miocene of East Africa. Palaeovertebrata 14: 117-200.

Coster P., Benammi M., Mahboubi M., Tabuce R., Adaci M., Marivaux L., Bensalah M., Mahboubi S., Mahboubi A., Mebrouk F., MaAmeri C. \& Jaeger J.-J. 2012. - Chronology of the Eocene continental deposits of Africa: magnetostratigraphy and biostratigraphy of the El Kohol and Glib Zegdou Formations. Geological Society of America Bulletin 124: 1590-1606. http:// dx.doi.org/10.1130/B30565.1

Court N. \& Hartenberger J.-L. 1992. - A new species of the hyracoid mammal Titanohyrax from the Eocene of Tunisia. Paleontology 35: 309-317.

CROCHet J.-Y. 1986. - Kasserinotherium tunisiense nov, gen., nov. sp., troisième marsupial découvert en Afrique (Éocène inférieur de Tunisie). Compte rendu de l'Académie des Sciences de Paris 302: 923-926.

CZAPlewski N. J., Morgan G. S. \& Mcleod S. A. 2008. — Chiroptera, in JANIS C. M., GuNNELL G. F. \& UHEN M. D. (eds), Evolution of Tertiary Mammals of North America. Vol. 2. Small Mammals, Xenarthrans, and Marine Mammals. Cambridge University Press, Cambridge: 174-197.

CZAPLEWSKI N. J. \& MORGAN G. S. 2012. - New basal noctilionoid bats (Mammalia: Chiroptera) from the Oligocene of subtropical North America, in GunNell G. F. \& Simmons N. B. (eds), Evolutionary History of Bats: Fossils, Molecules and Morphology. Cambridge University Press, Cambridge: 162-209.

DODSON P. \& WeXLAR D. 1979. - Taphonomic investigations of owl pellets. Paleobiology 5: 275-284.

Eick G. N., Jacobs D. S. \& Matthee C. A. 2005. - A nuclear DNA phylogenetic perspective on the evolution of echolocation and historical biogeography of extant bats (Chiroptera). Molecular Biology and Evolution 22 (9): 1869-1886. http://dx.doi. org $/ 10.1093 / \mathrm{molbev} / \mathrm{msi} 180$

EITING T. P. \& GUNNELL G. F. 2009. - Global completeness of the bat fossil record. Journal of Mammalian Evolution 16: 151-173. http://dx.doi.org/10.1007/s10914-009-9118-x

FilHOL H. 1877. - Recherches sur les phosphorites du Quercy. Étude des fossiles qu'on y rencontre, et specialement des Mammiferes. Annales des Sciences géologiques 7 (7): 1-220.

Fleming T. H. \& EBY P. 2003. - Ecology of bat migration, in Kunz T. H. \& FEnTON M. B. (eds), Bat Ecology. University of Chicago Press, Chicago: 156-208 
Fracasso M. P. A., Salles L. De O. \& Perini F. A. 2011. Upper molar morphology and relationships among higher taxa in bats. Journal of Mammalogy 92 (2): 421-432. http://dx.doi. org/10.1644/09-MAMM-A-415.1

GHEERBRANT E. \& HARTENBERGER J.-L. 1999. — Nouveau mammifère insectivore (?Lipotyphla, ?Erinaceomorpha) de l'Éocène inférieur de Chambi (Tunisie). Paläontologische Zeitschrift 73: 143-156. http://dx.doi.org/10.1007/BF02987988

GraY J. E. 1831. - Description of some new genera and species of bats. Zoological Miscellany 1831: 37, 38.

Gunnell G. F., Jacobs B. F., Herendeen P. S., Head J. J., Kowalski E., Msuya C. P., Mizambwa F. A., Harrison T., HABERSETZER J. \& STORCH G. 2003. — Oldest placental mammal from sub-Saharan Africa: Eocene microbat from Tanzania - evidence for early evolution of sophisticated echolocation. Palaeontologica Electronica 5: 1-10.

GunNell G. F. \& Simmons N. B. 2005. - Fossil evidence and the origin of bats. Journal of Mammalian Evolution 12: 209246. http://dx.doi.org/10.1007/s10914-005-6945-2

Gunnell G. F., Simmons E. L. \& Seiffert E. R. 2008. — New Bats (Mammalia: Chiroptera) from the Late Eocene and Early Oligocene, Fayum Depression, Egypt. Journal of Vertebrate Paleontology 28 (1): 1-11. http://www.jstor.org/stable/30126328

Gunnell G. F., Simmons N. B. \& Seiffert E. R. 2014. — New Myzopodidae (Chiroptera) from the late Paleogene of Egypt: emended family diagnosis and biogeographic origins of Noctilionoidea. Plos One 9 (2): e86712. http://dx.doi.org/10.1371/ journal.pone.0086712

Habersetzer J., Schlosser-Sturm E., Storch G. \& Sigé B. 2012. - Shoulder joint and inner ear of Tachypteron franzeni, an emballonurid bat from the Middle Eocene of Messel, in GunNell G. F. \& Simmons N. B. (eds), Evolutionary History of Bats: Fossils, Molecules and Morphology. Cambridge University Press, Cambridge: 67-104.

HAND S. 1998a. - Riversleigha williamsi gen. et sp. nov., a large Miocene hipposiderid (Microchiroptera) from Riversleigh, Queensland. Alcheringa 22: 259-276.

HAND S. 1998b. - Xenorhinos, a new genus of old world leafnosed bats (Microchiroptera: Hipposideridae) from the Australian Miocene. Journal of Vertebrate Paleontology 18: 430-439.

HaND S. \& KIRSCH J. A. W. 2003. - Archerops, a new annectent hipposiderid genus (Mammalia: Microchiroptera) from the Australian Miocene. Journal of Paleontology 77 (6): 11391151. http://dx.doi.org/10.1666/0022-3360(2003)077<113 9:AANAHG>2.0.CO;2

Hand S., Novacek M., Godthelp H. \& Archer M. 1994. First Eocene bat from Australia. Journal of Vertebrate Paleontology 14 (3): 375-381. http://www.jstor.org/stable/4523576

Hand S., Sigé B. \& MaITRE E. 2012. - Necromantis Weithofer, 1887, large carnivorous Middle and Late Eocene bats from the French Quercy Phosphorites: new data and unresolved relationships, in Gunnell G. F. \& Simmons N. B. (eds), Evolutionary History of Bats: Fossils, Molecules and Morphology. Cambridge University Press, Cambridge: 210-251.

HARTENBERGER J.-L. 1986. - Hypothèse paléontologique sur l'origine des Macroscelidea (Mammalia). Compte rendu de l'Académie des Sciences de Paris 5: 247-249.

Hartenberger J.-L. \& Marandat B. 1992. - A new genus and species of an Early Eocene primate from North Africa. Human Evolution 7: 9-16.

Hill J. E. \& Smith J. D. 1984. - Bats: a Natural History. Vol. 877. British Museum (Natural History) Publications, London, United Kingdom, 243 p.

Hill J. E., Zubaid A. \& Davison G. W. H. 2009. - The taxonomy of leaf-nosed bats of the Hipposideros bicolor group (Chiroptera: Hipposideridae) from southeastern Asia. Mammalia 50 (4): 535-540. http://dx.doi.org/10.1515/ mamm.1986.50.4.535
Hugueney M. 1984. - Verneuil (Allier), gisement de petits mammifères de l'Oligocène supérieur. Revue Scientifique du Bourbonnais 1984: 128-140.

KoOpMAn K. F. 1994. - Chiroptera: Systematics. Handbook of Zoology. Mammalia VIII 60: 1-217.

KOWALSKI K. 1995. - Taphonomy of bats (Chiroptera). Geobios 28 (1): 251-256. http://dx.doi.org/10.1016/S0016-6995(95)80172-3

Lack J. B., Roehrs Z. P., Stanley G. E., Manuel R. \& Van Den BussCHE R. A. 2010. - Molecular phylogenetics of Myotis indicate familial-level divergence for the genus Cistugo (Chiroptera). Journal of Mammalogy 91 (4): 976-992. http://dx.doi.org/10.1644/09MAMM-A-192.1

LAUDET F. \& SELVA N. 2005. - Ravens as small bone accumulators: first taphonomic study on mammal remains in raven pellets. Palaeogeography, Palaeoclimatology, Palaeoecology 226 (3-4): 272-286. http://dx.doi.org/10.1016/j.palaeo.2005.05.015

LECHE W. 1911. - Einige Dauertypen aus der Klasse der Saugetiere. Zoologischen Anzeiger 38: 551-559.

LEGENDRE R. S. 1980. - Un chiroptère emballonuridé dans le Néogène d'Europe occidentale; considération paléobiogéographiques. Geobios 13 (6): 839-847. http://dx.doi.org/10.1016/S00166995(80)80039-8

LEGENDRE R. S. 1984. - Étude odontologique des représentants actuels du groupe Tadarida (Chiroptera, Molossidae). Implications phylogénétiques, systématiques et zoogéographiques. Revue Suisse de Zoologie 91: 399-442.

MaITRE E. 2014. - Western European middle Eocene to early Oligocene Chiroptera: systematics, phylogeny and paleoecology based on new material from the Quercy (France). Swiss journal of Palaeontology 133: 141-242. http://dx.doi.org/10.1007/s13358-014-0069-3

Maitre E., Sigé B. \& EsCarguel G. 2008. - A new family of bats in the Paleogene of Europe: Systematics and implications for the origin of emballonurids and rhinolophoids. Neues Jahrbuch für Geologie und Paläontologie, Abhandlungen 250: 199-216. http:// dx.doi.org/10.1127/0077-7749/2008/0250-0199

Marandat B., Crochet J.-Y., Godinot M., Hartenberger J.-L., Legendre R. S., Remy J. A., Sigé B., Sudre J. \& Vianey-Liaud M. 1993. - Une nouvelle faune de mammifères d'âge éocène moyen dans les Phosphorites du Quercy. Geobios 26 (5): 617-623. http://dx.doi.org/10.1016/0016-6995(93)80042-P

Marivaux L., AdaCi M., Bensalah M., Rodrigues H. G., Hautier L., Mahboubi M., Mebrouk F., Tabuce R. \& Vianey-LiAUD M. 2011a. - Zegdoumyidae (Rodentia, Mammalia), stem anomaluroid rodents from the Early to Middle Eocene of Algeria (Gour Lazib, Western Sahara): new dental evidence. Journal of Systematic Palaeontology 9 (4): 563-588. http://dx.doi.org/10.10 80/14772019.2011.562555

Marivaux L., Tabuce R., Lebrun R., Ravel A., AdaCi M., Mahboubi M. \& BENSALAH M. 2011b. - Talar morphology of azibiids, strepsirhine-related primates from the Eocene of Algeria: phylogenetic affinities and locomotor adaptation. Journal of Human Evolution 61 (4): 447-457. http://dx.doi.org/10.1016/j.jhevol.2011.05.013 Marivaux L., Ramdarshan A., Essid E. M., Marzougui W., Ammar H. K., Lebrun R., Marandat B., Merzeraud G., Tabuce R. \& VIANEY-LIAUD M. 2013. — Djebelemur, a tiny pre-tooth-combed primate from the Eocene of Tunisia: a glimpse into the origin of crown strepsirhines. PLOS ONE 8 (12): e80778. http://dx.doi. org/10.1371/journal.pone.0080778

Mebrouk F., Mahboubi M., Bessedik M. \& Feist M. 1997. L'apport des charophytes à la stratigraphie des formations continentales paléogènes de l'Algérie. Geobios 30 (2): 171-177. http:// dx.doi.org/10.1016/S0016-6995(97)80221-5

Menu H. 1985. - Morphotypes dentaires actuels et fossiles des chiroptères vespertilioninés. 1ère partie: étude des morphologies dentaires. Palaeovertebrata 15 (2): 71-128.

Menu H. 1987. - Morphotypes dentaires actuels et fossiles des chiroptères vespertilioninés. 2 ème partie: implications systématiques et phylogénétiques. Palaeovertebrata 17 (3): 77-150. 
Menu H. \& Sigé B. 1971. — Nyctalodontie et myotodontie, important caractères de grades évolutifs chez les chiroptères entomophages. Comptes rendus de l'Académie des Sciences de Paris 272: 1735-1738.

Miller G. S. 1907. - The families and genera of bats. Bulletin of the United States National Museum 57: 1-282. http://dx.doi. org/10.5962/bhl.title.16306

MorGAN G. S. \& CZAPLEWSKI N. J. 2003. - A new bat (Chiroptera: Natalidae) from the Early Miocene of Florida, with comments on natalid phylogeny. Journal of Mammalogy 84 (2): 729-752. http://www.jstor.org/stable/1383917

Mourer-Chauviré C., Tabuce R., Mahboubi M., Adaci M. \& BENSAlaH M. 2011. - A phororhacoid bird from the Eocene of Africa. Naturewissenschaften 98 (10): 815-823. http://dx.doi. org/10.1007/s00114-011-0829-5

Page R. D. M. 2001. - NDE: NEXUS Data Editor 0.5. 0. University of Glasgow, Glasgow.

Pictet F. J., Gaudin C. \& HaRpe P. DE la 1855. - Mémoire sur les animaux vertébrés trouvés dans le terrain sidérolithique du Canton de Vaud. Kessmann, Genève: 27-115 (Matériaux pour la paléontologie suisse ; 1 ).

PococK T. N. 1987. - Adequacy, completeness and the fossil record, in Donovan S. K. \& Paul C. R. C. (eds), The Adequacy of the Fossil Record. John Wiley \& Sons, Chichester: 1-22.

Ravel A., Marivaux L., Tabuce R., Adaci M., Mahboubi M. Mebrouk F. \& Bensalah M. 2011. - The oldest African bat from the Early Eocene of El Kohol (Algeria). Naturwissenschaften 98 (5): 397-405. http://dx.doi.org/10.1007/s00114-011-0785-0

Ravel A., Marivaux L., Tabuce R., Ben Haj Ali M. \& Essid E. M. 2012. - A new large philisid (Mammalia, Chiroptera, Vespertilionoidea) from the late Early Eocene of Chambi, Tunisia. Palaeontology 55: 1035-1041. http://doi.org/10.1111/j.14754983.2012.01160.x

Ravel A., Marivaux L., QI T., Wang Y.-Q. \& BeARD K. C. 2014. New chiropterans from the middle Eocene of Shanghuang (Jiangsu Province, Coastal China): new insight into the dawn horseshoe bats (Rhinolophidae) in Asia. Zoologica Scripta 43 (1): 1-23. http://dx.doi.org/10.1111/zsc.12027

Ravel A., Adaci M., Bensalah M., Mahboubi M., Mebrouk F., Essid E. M., Marzougui W., Ammar H. K., Charruault A.-L., Lebrun R., Tabuce R., Vianey-Liaud M. \& Marivaux L. 2015. - New philisids (Mammalia, Chiroptera) from the Early-Middle Eocene of Algeria and Tunisia: new insight into the phylogeny, paleobiogeography and paleoecology of the Philisidae. Journal of Systematic Palaeontology 13 (8): 691-709. http://dx.doi. org/10.1080/14772019.2014.941422

Remy J. A., Crochet J.-Y., Sigé B., Sudre J., De Bonis L., Vianey-Liaud M., Godinot M., HArtenberger J.-L., LAnge-Badre B. \& Comte B. 1987. - Biochronologie des Phosphorites du Quercy: Mise à jour des listes fauniques et nouveaux gisements de mammiferes fossiles. Münchner Geowissenschaftliche Abhandlungen A 10: 169-188.

REVILLIOD P. 1917. - Contribution à l'étude des chiroptères des terrains tertiaires. Mémoires de la Société Paléontologique Suisse 43: $1-58$.

REvilliod P. 1920. - Contribution à l'étude des chiroptères des terrains tertiaires. Deuxième partie. Mémoires de la Société Paléontologique Suisse 44: 63-129.

REVILLIOD P. 1922. - Contribution à l'étude des chiroptères des terrains tertiaires. Troisième partie et fin. Mémoire de la Société Paléontologique Suisse 45: 131-195.

Russell D. E., Louis P. \& SAVAGE D. E. 1973. - Chiroptera and Dermoptera of the French Early Eocene. University of California Publications in Geological Sciences, vol. 95, 57 p.

SCHLosser M. 1887. — Die affen, Lemuren, Chiropteren, Insectivoren, Marsupialier, Creodonten und Carnivoren des europäischen Tertiärs und deren Beziehungen zu ihren lebenden und fossilen aussereuropäischen Verwandten. Beiträge zur paläontologie Österreich-Ungarns und des Orients 6(1-2): 1-224.
SIGÉ B. 1966. - Les chiroptères fossiles de Bouzigues (Hérault); recherches anatomiques sur Pseudorhinolophus bouziguensis $n$. $s p$., Thèse de $3^{\text {ème }}$ cycle, Paris (non publié), 248 p.

SigÉ B. 1968. — Les chiroptères du Miocène inférieur de Bouzigues, I. Étude systématique. Palaeovertebrata 1 (3): 65-133. http:// dx.doi.org/10.18563/pv.1.3.65-133

SIGÉ B. 1978. — La poche à phosphate de Ste-Néboule (Lot) et sa faune de vertébrés du Ludien supérieur. 8. Insectivores et Chiroptères. Palaeovertebrata 8 (2-4): 249-268.

SigÉ B. 1985. - Les chiroptères Oligocènes du Fayum, Égypte. Geologica et Palaeontologica 19: 161-189.

SIGÉ B. 1990. - Nouveaux chiroptères de l'Oligocène moyen des Phosphorites du Quercy, France. Compte rendu de l'Académie des Sciences de Paris 310: 1131-1137.

SigÉ B. 1991a. - Morphologie dentaire lactéale d'un chiroptère de l'Éocène inférieur-moyen d'Europe. Geobios 13: 231-236.

SIGÉ B. 1991b. - Rhinolophoidea et Vespertilionoidea (Chiroptera) du Chambi (Éocène inférieur de Tunisie). Aspect biostratigraphique, biogéographique et paléoécologique de l'origine des chiroptères modernes. Neues Jahrbuch für Geologie und Paläontologie, Abhandlungen 182: 355-376.

SigÉ B. 1995. - Le Garouillas et les sites contemporains (Oligocène, MP25) des Phosphorites du Quercy (Lot, Tarn-et-Garonne, France) et leurs faunes de vertébrés. 5. Chiroptères. Palaontographica Abteilung A 236: 77-124.

SigÉ B. 1997. — Les remplissages karstiques polyphasés (Éocène, Oligocène, Pliocène) de Saint-Maximin (phosphorite du Gard) et leur apport à la connaissance des faunes européennes, notamment pour l'Éocène moyen (MP13). 3. Systématique: euthériens entomophages, in AgUILAR J.-P., LEgENDRE R.-S. \& MiCHAUX J. (eds), Actes du Congrès BiochroM'97. Mémoires et Travaux de l'École pratique des Hautes Études, Institut de Montpellier Montpellier: 737-750.

SIGÉ B. 2011a. - Cryptobune nov. gen., chiroptère carnivore des Phosphorites du Quercy, SW France. Bulletin de la Société d'Histoire naturelle de Toulouse 147: 47-54.

SIGÉ B. 2011b. - A nycterid bat from the late Oligocene paleokarstic filings, Quercy, SW France, in LEHMANN T. \& SCHAAL S. F. K. (eds), The World at the Time of Messel. Volker Mosbrugger, Frankfurt: 155.

SigÉ B. \& LEGENDRE R. S. 1983. - L'histoire des peuplements de chiroptères du bassin méditerranéen: l'apport comparé des remplissages karstiques et des dépôts fluvio-lacustres. Mémoire Biospéologique 10: 209-225.

Sigé B., Hand S. \& ArCher M. 1982. - An Australian Miocene Brachipposideros (Mammalia, Chiroptera) related to Miocene representatives from France. Palaeovertebrata 12 (5): 149-172.

Sigé B., Aguilar J.-P. \& Marandat B. 1991. — Extension au Miocène inférieur des remplissages phosphatés du Quercy. La faune de vertébrés de Crémat (Lot, France). Geobios 24 (4): 497-502. http://dx.doi.org/10.1016/S0016-6995(06)80251-2

Sigé B., Thomas H., Sen S., Gheerbrant E., Roger J. \& AlSulaimani Z. 1994. - Les chiroptères de Taqah (Oligocène inférieur, Sultanat d'Oman). Premier inventaire systématique. Münchner Geowissenschaftliche Abhandlungen 26: 35-48.

Sigé B., Maitre E. \& Hand S. 2012. - Necromantodonty, the primitive condition of lower molars among bats, in Gunnell G. F. \& Simmons N. B. (eds), Evolutionary History of Bats: Fossils, Molecules and Morphology. Cambridge University Press, Cambridge: 456-469.

Simmons N. B. 2005a. - Chiroptera, in Rose K. D. \& ARCHIBALD J. D. (eds), The Rise of Placental Mammals: Origins and Relationships of the Major Extant Clades. The Johns Hopkins University Press Baltimore, Maryland: 159-173.

Simmons N. B. 2005b. - An Eocene Big Bang for bats. Science 307: 527-528. http://dx.doi.org/10.1126/science.1108871

SiMMONS N. B. \& GEISLER J. H. 1998. - Phylogenetic relationships of Icaronycteris, Archaeonycteris, Hassianycteris, and Palaeochirop- 
teryx to extant bat lineages, with comments on the evolution of echolocation and foraging strategies in Microchiroptera. Bulletin of the United States National Museum 235: 1-182. http://hdl. handle.net/2246/1629

Simmons N. B. \& ConwaY T. M. 2003. - Evolution of ecological diversity in bats, in Kunz T. H. \& Fenton M. B. (eds), Bat Ecology. The University of Chicago, Chicago: 493-535.

Simmons N. B., Seymour K. L., Habersetzer J. \& Gunnell G. F. 2008. - Primitive Early Eocene bat from Wyoming and the evolution of flight and echolocation. Nature 451: 818-821. http://dx.doi.org/10.1038/nature06549

Simmons N. B., Seymour K. L., Habersetzer J. \& GunNell G. F. 2010. - Inferring echolocation in ancient bats. Nature 466 : E8. http://dx.doi.org/10.1038/nature09219

Smith T., Rana R. S., Missiaen P., Rose K. D., Sahni A., Singh H. \& SingH L. 2007. — High bat (Chiroptera) diversity in the Early Eocene of India. Naturwissenschaften 94: 1003-1009. http://dx.doi.org/10.1007/s00114-007-0280-9

Smith T., Habersetzer J., Simmons N. B. \& Gunnell G. F. 2012. - Systematics and paleobiogeography of early bats, in GUNNELl G. F. \& Simmons N. B. (eds), Evolutionary History of Bats: Fossils, Molecules and Morphology. Cambridge University Press, Cambridge: 23-66.

Storch G., Sigé B. \& Habersetzer J. 2002. - Tachypteron franzeni n. gen., n. sp., earliest emballonurid bat from Middle Eocene of Messel (Mammalia, Chiroptera). Paläontologische Zeitschrift 76: 189-199. http://dx.doi.org/10.1007/BF02989856

SUdRE J. 1979. - Nouveaux mammifères éocènes du Sahara occidental. Palaeovertebrata 9 (3): 83-115.

Sudre J., Sigé B., Remy J. A., Marandat B., Hartenberger J.-L., GODINOT M. \& CROCHET J.-Y. 1990. - Une faune de niveau d'Egerkingen (MP 14; Bartonien inférieur) dans les Phosphorites du Quercy (Sud de la France). Palaeovertebrata 20 (1): 1-32.

SwOFFORD D. L. 2002. - PAUP - Phylogenetic Analysis Using Parsimony ( ${ }^{*}$ and Other Methods), Version 4.0. Sinauer Associates, Sunderland, Massachusetts: $128 \mathrm{p}$.

SZALAY F. S. 1969. - Mixodectidae, Microsyopidae, and the insectivore-primate transition. Bulletin of American Museum of Natural History 140 (4): 163-330. http://hdl.handle.net/2246/1130

Tabuce R., Adnet S., CappetTa H., Noubhani A. \& Quillevere F. 2005. - Aznag (bassin d'Ouarzazate, Maroc), nouvelle localité à sélaciens et mammiferes de l'Éocène moyen (Lutétien) d'Afrique. Bulletin de la Société géologique de France 176 (4): 381-400.

Tabuce R., Marivaux L., Adaci M., Bensalah M., HartenBerger J.-L., MAhboubi M., Mebrouk F., TAFForeau P. \& JAEger J.-J. 2007. - Early Tertiary mammals from North Africa reinforce the molecular Afrotheria clade. Proceeding of the Royal Society $B$ 274: 1159-1166. http://dx.doi.org/10.1098/rspb.2006.0229

TABUCE R., ANTUNes M. T. \& SigÉ B. 2009a. - A new primitive bat from the earliest Eocene of Europe. Journal of Vertebrate Paleontology 29 (2): 627-630. http://dx.doi.org/10.1671/039.029.0204

Tabuce R., Marivaux L., Lebrun R., Adaci M., Bensalah M., Fabre P.-H., Fara E., Gomez-Rodriguez H., Hautier L., Jaeger J.-J., Lazzari V., Mebrouk F., Peigné S., Sudre J., TafForeau P., Valentin X. \& Mahboubi M. 2009b. - Anthropoid versus strepsirhine status of the African Eocene primates Algeripithecus and Azibius: craniodental evidence. Proceeding of the Royal Society B 276: 4087-4094. http://dx.doi.org/10.1098/ rspb.2009.1339

Tabuce R., Charruault A.-L., Adaci M., Bensalah M., Ben Haj Ali M., Essid E. M., Marivaux L., Vianey-Liaud M. \& Mahboubi M. 2011. - The early Eocene radiation of Hyracoidea (Mammalia, Afrotheria): new fieldwork evidence from northwestern Africa, in LEHMANN T. \& SCHAAL S. F. K. (eds), The World at the Time of Messel. Senckenberg Research Institute and Natural History Museum Frankfurt: 161, 162.

TEELING E. C. 2009. - Hear, hear: the convergent evolution of echolocation in bats? Trends in Ecology and Evolution 24: 351354. http://dx.doi.org/10.1016/j.tree.2009.02.012

Teeling E. C., Springer M. S., Madsen O., Bates P., O'Brien S. J. \& MURPHY W. J. 2005. - A molecular phylogeny for bats illuminates biogeography and the fossil record. Science 307: 580584. http://dx.doi.org/10.1126/science.1105113

TeEling E. C., Dool S. \& Springer M. S. 2012. — Phylogenies, fossils and functional genes: the evolution of echolocation in bats, in GuNNELl G. F. \& Simmons N. B. (eds), Evolutionary History of Bats: Fossils, Molecules and Morphology. Cambridge University Press, New York: 1-22.

Tejedor M. F., CZaplewski N. J., Goin F. J. \& Aragon E. 2005. The oldest record of South American bats. Journal of Vertebrate Paleontology 25: 990-993. http://www.jstor.org/stable/4524527

Van Cakenberghe V. \& De Vree F. 1985. - Systematic of African Nycteris (Mammalia: Chiroptera), in SCHUCHMANN K.-L. (ed.), Proceedings of the International Symposium on African Vertebrates: Systematics, Phylogeny and Evolutionary Ecology. Zoologisches Forschingsinstitut und Museum Alexander Koenig, Bonn: 53-90.

VAN CAKENBERGHE V. \& De VREe F. 1993a. - The systematic status of southeast Asian Nycteris (Chiroptera: Nycteridae). Mammalia 57: 227-244. http://dx.doi.org/10.1515/mamm.1993.57.2.227

Van Cakenberghe V. \& De VRee F. 1993b. - Systematics of African Nycteris (Mammalia: Chiroptera). Part II. The Nycteris hispida group. Bonner Zoologische Beiträge 44: 299-332.

VAN CAKENBERGHe V. \& De VREE F. 1998. - Systematics of African Nycteris (Mammalia: Chiroptera). Part III. The Nycteris thebaica group. Bonner Zoologische Beiträge 48: 123-166.

VAN VALEN L. M. 1966. - Deltatheridia, a new order of mammals. Bulletin of the American Museum of Natural History 132:1-125. http://hdl.handle.net/2246/1126

VAN VALEN L. 1979. - The Evolution of Bat. Evolutionary Theory 4: 103-121.

Vianey-Liaud M., Jaeger J.-J., Hartenberger J.-L. \& MahBOUBI M. 1994. - Les rongeurs de l'Éocène d'Afrique NordOccidentale (Glib Zegdou, Algérie; Chambi, Tunisie) et l'origine des Anomaluridae. Paleovertebrata 23 (1-4): 93-118.

Watrous L. E. \& WheEler Q. D. 1981. - The out-group comparison method of character analysis. Systematic Biology 30 (1) 1-11. http://dx.doi.org/10.1093/sysbio/30.1.1

WeITHOFER A. 1887. - Zur kenntniss der fossilen cheiropteren der französischen Phosphorite. Mathematisch-naturwissenschaftlich Classe 96: 341-360.

WingE H. 1893. — Jordfundne og nulevende Flagermus fra Lagoa Santa Minas Geraes, Brasilien: med udsigt over flagermusenes indbyrdes slaegtskab. Museo lundii 2: 92 p. 


\section{ANNEXES}

Matrices et descriptions des caractères des analyses cladistiques réalisées dans le but de déterminer les positions phylogénétiques des Hipposideridae de Chambi (Annexe 1) et des Emballonuridae et des Nycteridae de Chambi et du Glib Zegdou (Annexe 2).

ANNEXE 1. - Matrice de caractères utilisée pour l'analyse phylogénétique incluant les Hipposideridae et les Rhinolophidae. Les auteurs des taxons sont cités dans la dernière partie de l'Annexe 1.

\begin{tabular}{|c|c|c|c|c|c|c|c|c|c|c|c|c|c|c|c|c|c|c|c|c|}
\hline Taxon & 1 & 2 & 3 & 4 & 5 & 6 & 7 & 8 & 9 & 10 & 11 & 12 & 13 & 14 & 15 & 16 & 17 & 18 & 19 & 20 \\
\hline Icaronycteris menui & ? & ? & 0 & 0 & $?$ & 0 & ? & $?$ & 0 & 0 & 0 & 0 & 0 & 0 & 0 & 0 & 0 & 0 & 0 & 0 \\
\hline Archaeonycteris brailloni & 0 & ? & ? & ? & ? & ? & ? & ? & 0 & 0 & 0 & 0 & 0 & 0 & 0 & 0 & 0 & 0 & 0 & 0 \\
\hline Palaeochiropteryx tupaiodon & 0 & 1 & 0 & 0 & 0 & 0 & 1 & 0 & 0 & 0 & 1 & 0 & 0 & 0 & 0 & 0 & 0 & 0 & 1 & 0 \\
\hline Vaylatsia frequens & 1 & 1 & 1 & 1 & 1 & 1 & ? & ? & 0 & 1 & 0 & 0 & 1 & 1 & 0 & 1 & 1 & 0 & 1 & 1 \\
\hline Rhinolophus ferrumequinum & 1 & 0 & 0 & 1 & 1 & 1 & 1 & 0 & 0 & 0 & 1 & 0 & 2 & 1 & 1 & 1 & 0 & 0 & 1 & 1 \\
\hline Rhinolophus hipposideros & 1 & 0 & 1 & 1 & 1 & 1 & 1 & 0 & 0 & 0 & 1 & 0 & 2 & 1 & 1 & 1 & 1 & 0 & 0 & 1 \\
\hline Hipposideros lankadiva & 0 & 1 & 0 & 1 & 1 & 1 & 0 & 0 & 0 & 1 & 1 & 1 & - & - & 1 & 1 & 0 & 1 & 0 & 1 \\
\hline Hipposideros bicolor & 0 & 1 & 0 & 1 & 1 & 1 & 0 & 0 & 0 & 0 & 1 & 1 & - & - & 1 & 1 & 1 & 1 & 0 & 1 \\
\hline Hipposideros (Pseudorhinolophus) schlosseri & 0 & ? & 1 & 1 & 0 & 1 & ? & ? & 0 & 0 & 0 & 1 & - & - & 1 & 1 & 0 & 1 & 0 & 1 \\
\hline Hipposideros (Pseudorhinolophus) africanum & ? & ? & ? & ? & ? & ? & ? & ? & 1 & 0 & ? & ? & ? & ? & ? & ? & ? & ? & ? & 1 \\
\hline Hipposideros (Pseudorhinolophus) bouziguensis & ? & ? & 0 & 0 & ? & 1 & 1 & 0 & 0 & 0 & 0 & 1 & - & - & 0 & 1 & 0 & 1 & 0 & 1 \\
\hline Palaeophyllophora oltina & 0 & 1 & 0 & 0 & 0 & 1 & ? & ? & 0 & 1 & 0 & 0 & 2 & 1 & 0 & 1 & 1 & 0 & 1 & 1 \\
\hline Palaeophyllophora? tunisiensis Ravel, n. sp. & ? & ? & ? & ? & ? & ? & ? & ? & ? & ? & 0 & ? & $?$ & $?$ & 1 & 1 & 0 & 0 & 1 & 1 \\
\hline Palaeophyllophora quercyi & 0 & 1 & 0 & 0 & 0 & 1 & 1 & 0 & 0 & 1 & 0 & 0 & 2 & 1 & 0 & 1 & $0+1$ & 1 & 1 & 1 \\
\hline Protorhinolophus shanghuangensis & 1 & 1 & 1 & 0 & 1 & 1 & ? & ? & 1 & 1 & 0 & 0 & 1 & 0 & 0 & 1 & 1 & 0 & 1 & 1 \\
\hline
\end{tabular}

\section{Taxon}

$\begin{array}{llllllllllllllllllll}21 & 22 & 23 & 24 & 25 & 26 & 27 & 28 & 29 & 30 & 31 & 32 & 33 & 34 & 35 & 36 & 37 & 38 & 39 & 40\end{array}$

lcaronycteris menui

Archaeonycteris brailloni

Palaeochiropteryx tupaiodon

Vaylatsia frequens

Rhinolophus ferrumequinum

Rhinolophus hipposideros

Hipposideros lankadiva

Hipposideros bicolor

Hipposideros (Pseudorhinolophus) schlosseri

Hipposideros (Pseudorhinolophus) africanum

Hipposideros (Pseudorhinolophus) bouziguensis

Palaeophyllophora oltina

Palaeophyllophora? tunisiensis Ravel, n. sp.

Palaeophyllophora quercyi

Protorhinolophus shanghuangensis

$\begin{array}{cccccccccccccccccccc}0 & 0 & 0 & 0 & 0 & 0+1 & 0 & 0 & 0 & 0 & 0 & 0 & 0 & 0 & 0+1 & 0 & 0 & 0 & 0 & 0 \\ ? & 0 & ? & 0 & 0 & 0+1 & 0 & ? & 0 & 0 & 0 & 0 & 1 & 0 & 0 & 0 & 0 & 0 & 0 & 0 \\ 0 & 0 & 1 & 1 & 0+1 & 1 & 0 & 0 & 0 & 0 & 0 & 1 & 0 & 0 & 1 & 0 & 1 & 0 & 1 & 0 \\ 0 & 1 & 1 & 0 & 0 & 1 & 1 & 0 & 0 & 0 & 0 & 0 & 1 & 1 & 0 & 0 & 0 & 0 & 1 & 1 \\ 0 & 1 & 1 & 0 & 1 & 2 & 1 & 0 & 0 & 0 & 0 & 1 & 1 & 1 & 1 & 0 & 1 & 0 & 1 & 1 \\ 0 & 1 & 1 & 0 & 1 & 2 & 1 & 0 & 0 & 0 & 0 & 0 & 1 & 1 & 0 & 0 & 0 & 0 & 1 & 1 \\ 0 & 1 & 0 & 0 & 1 & 2 & 0 & 1 & 1 & 0 & 1 & 1 & 0 & 0 & 0 & 1 & 1 & 1 & 1 & 1 \\ 1 & 1 & 0 & 1 & 1 & 2 & 1 & 1 & 1 & 0 & 0 & 1 & 0 & 0 & 1 & 1 & 0 & 1 & 1 & 1 \\ 0 & 1 & 0 & 0 & 1 & 2 & 0 & 0 & 1 & 0 & 1 & 0 & 0 & 0 & 1 & 1 & 1 & 0 & 1 & 1 \\ 0 & 1 & 0 & 0 & 1 & 1+2 & 0 & 1 & 1 & 0 & 1 & ? & ? & ? & ? & ? & ? & 0 & 1 & 1 \\ 1 & 1 & 0 & 0 & 1 & 2 & 0 & 1 & 1 & 0 & 0 & 1 & 0 & 0 & 1 & 1 & 1 & 0 & 1 & 2 \\ 1 & 1 & 0 & 2 & 0 & 0 & 0 & 1 & 0 & 0 & 1 & ? & 1 & 0 & 1 & 1 & 0 & 0 & 0 & 0 \\ 1 & 1 & 0 & 2 & 0 & 0 & 0 & 1 & 1 & 0 & 0 & ? & ? & ? & ? & ? & ? & 0 & 0 & 0 \\ 1 & 1 & 0 & 2 & 0 & 0 & 0 & 1 & 1 & 0 & 1 & 1 & 1 & 0 & 1 & 1 & 1 & 0 & 0 & 0 \\ 0 & 1 & 1 & 0 & 0 & 1 & 0 & 1 & 0 & 0 & 0 & ? & 1 & 1 & 0 & 0 & 0 & 0 & 0 & 0\end{array}$

\section{Taxon}

$\begin{array}{llllllllllllllllllll}41 & 42 & 43 & 44 & 45 & 46 & 47 & 48 & 49 & 50 & 51 & 52 & 53 & 54 & 55 & 56 & 57 & 58 & 59 & 60\end{array}$

Icaronycteris menui

Archaeonycteris brailloni

Palaeochiropteryx tupaiodon

Vaylatsia frequens

Rhinolophus ferrumequinum

Rhinolophus hipposideros

Hipposideros lankadiva

Hipposideros bicolor

Hipposideros (Pseudorhinolophus) schlosseri

Hipposideros (Pseudorhinolophus) africanum

Hipposideros (Pseudorhinolophus) bouziguensis

Palaeophyllophora oltina

Palaeophyllophora? tunisiensis Ravel, n. sp.

Palaeophyllophora quercyi

Protorhinolophus shanghuangensis

$\begin{array}{cccccccccccccccccccc}0 & 0 & 0 & 0 & 0 & 0 & 0 & 0 & 0 & 0 & 0 & 0 & 0 & 0 & 0 & 0 & 0 & 0 & 0 & 0 \\ 0 & 0 & 0 & 0 & 0 & 0 & 0 & 0 & 0+1 & 0 & 0 & 0 & 0 & 0 & 0 & 0 & ? & ? & ? & ? \\ 0 & 0 & 0 & 0 & 0 & 0 & 0 & 0 & 0 & 0 & 0 & 0 & 0 & 0 & 1 & 1 & 0 & 0 & 0 & 0 \\ 1 & 1 & 1 & 1 & 0+1 & 0+1 & 0 & 0+1 & 0 & 0 & 1 & 0 & 1 & 0 & 0 & 1 & 0 & 1 & 0 & 0 \\ 1 & 1 & 1 & 1 & 1 & 1 & 0 & 0 & 0 & 0 & 1 & 0 & 1 & 1 & 0 & 1 & 0 & 1 & 0 & 0 \\ 1 & 1 & 1 & 1 & 1 & 1 & 0 & 0 & 0 & 0 & 1 & 0 & 1 & 1 & 0 & 1 & 0 & 1 & 0 & 0 \\ 1 & 1 & 1 & 1 & 1 & 1 & 0 & 0 & 1 & 0 & 1 & 1 & 0 & 0 & 1 & 1 & 2 & 1 & 1 & 1 \\ 1 & 1 & 1 & 1 & 1 & 1 & 0 & 0 & 1 & 0 & 1 & 1 & 0 & 0 & 0 & 1 & 1 & 1 & 0 & 1 \\ 0 & 1 & 1 & 1 & 1 & 1 & 0 & 0 & 1 & 0+1 & 0 & 0 & 0 & 0+1 & 0 & 1 & 2 & 0 & 0 & 1 \\ 0 & 1 & 1 & 1 & 1 & 1 & 0 & 0 & 1 & 0 & 1 & 0 & 1 & 0 & 0 & 1 & 1 & 0 & 0 & 1 \\ 0 & 1 & 1 & 1 & 1 & 1 & 0 & 0 & 1 & 0+1 & 0 & 0 & 0 & 0 & 0 & 1 & 2 & 0 & 0+1 & 1 \\ 0 & 0 & 0 & 0 & 1 & 1 & 1 & 0 & 1 & 1 & 1 & 0 & 0 & 0 & 0 & 0 & 2 & 0 & 1 & 1 \\ 0 & 0 & 0 & 0 & 1 & 1 & 1 & 0 & 1 & 0 & 0 & 1 & 0 & 0 & 0 & 1 & ? & ? & ? & ? \\ 0 & 0 & 0 & 0 & 1 & 1 & 1 & 0 & 1 & 1 & 1 & 0 & 0 & 0 & 0 & 0 & 2 & 0 & 1 & 1 \\ 0+1 & 1 & 0 & 1 & 0+1 & 0+1 & 0 & 0+1 & 0 & 0 & 0 & 0 & 1 & 0 & 0 & 0 & 2 & 0 & 1 & 1\end{array}$




\section{Taxon}

Icaronycteris menui

Archaeonycteris brailloni

Palaeochiropteryx tupaiodon

Vaylatsia frequens

Rhinolophus ferrumequinum

Rhinolophus hipposideros

Hipposideros lankadiva

Hipposideros bicolor

Hipposideros (Pseudorhinolophus) schlosseri

Hipposideros (Pseudorhinolophus) africanum

Ravel, n. sp.

Hipposideros (Pseudorhinolophus) bouziguensis

Palaeophyllophora oltina

Palaeophyllophora? tunisiensis Ravel, n. sp.

Palaeophyllophora quercyi

Protorhinolophus shanghuangensis

$\begin{array}{llllllllllllllllllll}61 & 62 & 63 & 64 & 65 & 66 & 67 & 68 & 69 & 70 & 71 & 72 & 73 & 74 & 75 & 76 & 77 & 78 & 79 & 80\end{array}$

$\begin{array}{lllllllllllllllllllll}0 & ? & ? & ? & ? & ? & ? & ? & ? & ? & ? & ? & ? & ? & ? & ? & ? & ? & ? & ? \\ ? & ? & ? & ? & ? & ? & ? & ? & ? & ? & ? & ? & ? & ? & ? & ? & ? & ? & ? & ? \\ 0 & ? & ? & ? & ? & ? & ? & ? & ? & ? & ? & ? & ? & ? & ? & ? & ? & ? & ? & ? \\ 0 & ? & ? & ? & ? & ? & ? & ? & ? & ? & ? & ? & ? & ? & ? & ? & ? & ? & ? & ? \\ 0 & 0 & 0 & 0 & 0 & 0 & 1 & 0 & 1 & 0 & 0 & 2 & 1 & 1 & 1 & 0 & 1 & 2 & 0 & 1 \\ 0 & 0 & 0 & 0 & 0 & 0 & 1 & 0 & 1 & 0 & 0 & 2 & 1 & 1 & 1 & 0 & 1 & 2 & 0 & ? \\ 0 & 1 & 1 & 1 & 1 & 0 & 0 & 0 & 0 & 1 & 0 & ? & 0 & 0 & 1 & 0 & 1 & 0 & 2 & 0 \\ ? & ? & ? & ? & ? & ? & ? & ? & ? & ? & ? & ? & ? & ? & ? & ? & ? & ? & ? & ? \\ 1 & ? & ? & ? & ? & ? & ? & ? & ? & ? & ? & ? & ? & ? & ? & ? & ? & ? & ? & ? \\ 0 & ? & ? & ? & ? & ? & ? & ? & ? & ? & ? & ? & ? & ? & ? & ? & ? & ? & ? & ?\end{array}$

$\begin{array}{clllllllllllllllllllll}0+1 & 1 & 1 & 1 & 1 & 0 & 0 & 0 & 0 & 0 & 0 & 0 & 0 & 0 & 1 & 0 & 1 & 0 & 1 & 0 \\ 1 & ? & ? & ? & ? & ? & ? & ? & ? & ? & ? & ? & ? & ? & ? & ? & ? & ? & ? & ? \\ ? & ? & ? & ? & ? & ? & ? & ? & ? & ? & ? & ? & ? & ? & ? & ? & ? & ? & ? & ? \\ 1 & ? & ? & ? & 1 & 1 & 0 & 0 & 0 & ? & ? & 1 & 0 & 0 & 1 & 1 & ? & 1 & 2 & 0 \\ 1 & ? & ? & ? & ? & ? & ? & ? & ? & ? & ? & ? & ? & ? & ? & ? & ? & ? & ? & ?\end{array}$

Taxon $\begin{array}{llllllllllllllllllll}81 & 82 & 83 & 84 & 85 & 86 & 87 & 88 & 89 & 90 & 91 & 92 & 93 & 94 & 95 & 96 & 97 & 98 & 99 & 100\end{array}$

Icaronycteris menui

Archaeonycteris brailloni

Palaeochiropteryx tupaiodon

Vaylatsia frequens

Rhinolophus ferrumequinum

Rhinolophus hipposideros

Hipposideros lankadiva

Hipposideros bicolor

Hipposideros (Pseudorhinolophus) schlosseri

Hipposideros (Pseudorhinolophus) africanum

Ravel, n. sp.

Hipposideros (Pseudorhinolophus) bouziguensis

Palaeophyllophora oltina

Palaeophyllophora? tunisiensis Ravel, n. sp.

Palaeophyllophora quercyi

Protorhinolophus shanghuangensis

$\begin{array}{llllllllllllllllllllll}? & ? & ? & ? & ? & ? & ? & ? & ? & ? & ? & ? & ? & ? & ? & ? & ? & ? & ? & ? \\ ? & ? & ? & ? & ? & ? & ? & ? & ? & ? & ? & ? & ? & ? & ? & ? & ? & ? & ? & ? \\ ? & ? & ? & ? & ? & ? & ? & ? & ? & ? & ? & ? & ? & ? & ? & ? & ? & ? & ? & ? \\ ? & ? & ? & ? & ? & ? & ? & ? & ? & ? & ? & ? & ? & ? & ? & ? & ? & ? & ? & ? \\ 0 & 0 & 0 & 0 & 0 & 0 & 1 & 0 & 0 & 2 & 0 & 1 & 1 & 1 & 0 & 4 & 0 & 0 & 0 & 0 \\ 0 & 0 & 0 & 0 & 0 & ? & 1 & 0 & ? & 2 & 0 & 1 & 1 & 1 & 0 & 4 & 0 & 0 & 0 & 0 \\ 1 & 1 & 1 & 1 & 1 & 4 & 1 & 1 & 0 & 1 & 1 & 0 & 1 & 0 & 0 & 1 & 0 & 0 & 1 & 1 \\ ? & ? & ? & ? & ? & ? & ? & ? & ? & ? & ? & ? & ? & ? & ? & ? & ? & ? & ? & ? \\ ? & ? & ? & ? & ? & ? & ? & ? & ? & ? & ? & ? & ? & ? & ? & ? & ? & ? & ? & ? \\ ? & ? & ? & ? & ? & ? & ? & ? & ? & ? & ? & ? & ? & ? & ? & ? & ? & ? & ? & ?\end{array}$

Taxon

101102103104105

Icaronycteris menui Russell, Louis \& Savage, 1973

Archaeonycteris brailloni Russell, Louis \& Savage, 1973

Palaeochiropteryx tupaiodon Revilliod, 1917

Vaylatsia frequens Maitre, 2014

Rhinolophus ferrumequinum Schreber, 1774

Rhinolophus hipposideros Bechstein, 1800

Hipposideros lankadiva Kelaart, 1850

Hipposideros bicolor Temminck, 1834

Hipposideros (Pseudorhinolophus) schlosseri Revilliod, 1917

Hipposideros (Pseudorhinolophus) africanum Ravel, n. sp.

Hipposideros (Pseudorhinolophus) bouziguensis Sigé, 1968

Palaeophyllophora oltina Delfortrie, 1873

Palaeophyllophora? tunisiensis Ravel, n. sp.

Palaeophyllophora quercyi Revilliod, 1917

Protorhinolophus shanghuangensis Ravel, Marivaux, Qi, Wang \& Beard, 2014 


\section{OS DENTAIRE}

1. Processus coronoïde

0 . haut (approximativement deux fois la hauteur de la rangée dentaire)

1. bas

2. Apex du processus coronoïde
0 . arrondi
1. pointu

3. Prémolaires inférieures

0 . alignées avec l'axe des molaires

1. oblique par rapport à l'axe des molaires

4. Hauteur moyenne du ramus horizontal

0 . robuste (hauteur plus importante que la hauteur de la rangée dentaire)

1. gracile (moins haut que la hauteur de la rangée dentaire)

5. Angle du processus coronoïde

0 . quasi perpendiculaire au ramus horizontal

1. incliné distalement

6. Nombre d'incisives inférieures sur chaque mandibule

0 . trois incisives inférieures sur chaque mandibule

1. deux incisives inférieures sur chaque mandibule

\section{DENTURE INFÉRIEURE}

7. Taille relative des incisives inférieures

0 . i2 significativement plus grande qu' i1

1. i1 et i2 de taille équivalente

8. i1

0. trilobée

1. bilobée

9. Cingulum buccal de c1

0 . bien marqué

1. très léger ou absent

10. Tubercule de $\mathbf{c l}$

0 . quasi droit

1. incliné distalement

11. Réduction de $\mathrm{p} 2$

0 . modérément réduite

1. très réduite (moins de la moitié de la hauteur de p4)

2. non réduit ( $\mathrm{p} 2$ équivaut en largeur la $\mathrm{p} 4$ )

12. $\mathrm{p} 3$
0 . présente
1. absente

13. Développement de la p3

0 . bien développée (plus large que la p2)

1. très réduite (non fonctionnelle)

2. résiduelle

14. Nombre de racines à la p3

0 . deux

1. une
15. Tubercule distolingual sur $\mathrm{p} 4$

0 . présent

1. absent

16. Tubercule mésiolingual sur $\mathrm{p} 4$

0 . présent

1. absent

17. Méplat linguomésial sur p4

0 . absent

1. présent

18. Bassin du talonide de $\mathrm{p} 4$

0 . étendu dans le sens mésiodistal

1. très réduit

19. Bord buccal de $\mathrm{p} 4$

0 . non fléchi

1. fléchi

20. Triangle du trigonide de $\mathbf{m} 1$

0 . compressé mésiodistalement

1. ouvert lingualement

21. Triangle du trigonide de $\mathrm{m} 2$

0 . compressé mésiodistalement

1. ouvert lingualement

22. Distance entre le paraconide et le métaconide sur m $1-2$

0 . inférieure à la distance entre le métaconide et l'entoconide

1. égale ou supérieure à la distance entre le métaconide et l'entoconide

23. Distance entre le paraconide et le métaconide sur $\mathrm{m} 1-2$

0 . égale ou supérieure à la distance entre le métaconide et l'entoconide

1. inférieureà la distance entre le métaconide et l'entoconide

24. Largeur du talonide des m1-2

0 . largeur du talonide équivalente à celle du trigonide

1. talonide plus large que le trigonide

2. talonide moins large que le trigonide

25. Courte crête connectant l'hypoconulide à l'entoconide 0 . présente

1. absente

26. Position de l'hypoconulid

0 . hypoconulide centré entre l'hypoconide et l'entoconide

1. hypoconulide déplacé lingualement par rapport à l'entoconide

2. hypoconulide très proche et en position distobuccale par rapport à l'entoconide

27. Entocristide

0 . droite

1. incurvée 
28. Largeur du talonide de $\mathrm{m} 3$

0 . largeur du talonide équivalente à celle du trigonide

1. talonide moins large que le trigonide

DENTURE SUPÉRIEURE

29. Cingulum buccal de $\mathrm{C} 1$
0 . bien marqué
1. très léger à absent

30. Cuspide accessoire distale sur $\mathrm{C} 1$

0 . absente

1. présente

31. Tubercle de C1

0 . légèrement incliné distalement

1. fortement inclinée distalement

32. Position de P2

0 . centrée sur le même axe que la rangée dentaire

1. déplacée buccalement par rapport à la rangée dentaire

33. Couronne de P4

0 . non cintrée mésiodistalement

1. cintrée mésiodistalement

34. Bassin lingual de P4

0 . réduit

1. étiré lingualement

35. Tubercule mésiolingual sur P4

0 . présent

1. absent

36. Lobe mésiobuccal sur P4

0 . présent

1. absent

37. Postparacrista de $\mathbf{P} 4$

0 . légèrement incurvée buccodistalement

1. franchement inclinée buccodistalement

38. Couronne de M1-2

0 . cintrée mésiodistalement

1. non cintrée mésiodistalement

39. Ectoflexus sur M1-2

0 . simple

1. double

40. Cingulum buccal de M1-2
0 . continu
1. discontinu
2. absent

41. Échancrure mésiobuccale entre le parastyle et le précingulum sur M1-2

0 . légère ou absente

1. profonde

42. Centrocrêtes de l'ectolophe

0 . très courtes par rapport aux crêtes latérales (i.e., préparacrête et postmétacrête)

1. bien développées
43. Position du mésostyle

0 . en retrait par rapport au bord buccal

1. projeté buccalement

44. Mésostyle

0 . fortement réduit

1. bien développé

45. Paralophe sur M1-2

0 . présent

1. absent

46. Métalophe sur M1-2

0 . présent

1. absent

47. Protofosse

0 . étendue entre le paracône et le métacône

1. pas d'extension buccale

48. Hypocône

0 . absent

1. présent

49. Extension de la postprotocrête sur M1-2

0 . rejoint le postcingulum

1. ne rejoint ni le postcingulum ni le cingulum lingual

2. rejoint le cingulum lingual

50. Extension de la préprotocrête

0 . se connecte au précingulum

1. ne rejoint pas le précingulum

51. Précingulum

0 . large

1. fin

2. discontinu

52. Postcingulum

0 . non connecté au cingulum lingual

1. connecté au cingulum lingual

53. Extension du cingulum lingual

0 . continue

1. discontinue

54. Cingulum lingual

0 . épais

1. fin

55. Talon de la M1

0 . fortement projeté distolingualement

1. modérément projeté

56. Talon de la M2

0 . fortement projeté distolingualement

1. modérément projeté (significativement moins étiré que sur M1)

57. Métacône sur M3

0 . bien développé

1. fortement réduit

2. absent 
58. Échancrure mésiobuccale entre le parastyle et le précingulum sur M1-2

0 . légère ou absente

1. profonde

59. Prémétacrête sur M3

0 . présente

1. absent

60. Cingulum lingual de M3

0 . présent

1. absent

61. Bord buccal de M3

0 . infléchi

1. rectiligne

CARACTÈRES CRÂNIENS ET POSTCRÂNIENS

ISSUS DE HAND \& KIRSCH (2003)

62. Pelvis

0 . sans foramen postacétabulaire

1. avec foramen postacétabulaire postacetabular foramen

63. Nombre de phalanges sur les doigts $2-5$

0.3

1. 2

64. ceinture pectorale

0 . dernière vertèbre cervicale et première dorsale fusionnées avec les côtes et le sternum

1. modification plus prononcée, dernière vertèbre cervicale et les deux premières dorsales fusionnées avec les côtes et le sternum

2. aucune fusion entre la vertèbre cervicale et les dorsales

65. Région interorbitaire

0 . seulement modérément comprimée (largeur supérieure ou égale à la moitié de la largeur du rostre)

1. sensiblement conprimée (largeur inférieure à moitié de la largeur du rostre)

2. très nettement comprimée (largeur très nettement inférieure à la moitié de la largeur du rostre)

3. peu comprimée (largeur supérieure à la moitié de la largeur du rostre)

66. Largeur du rostre

0 . large (supérieure à la moitié de la largeur du mastoïde)

1. étroit (inférieure ou égale à la moitié de la largeur maximale de la boîte crânienne)

67. Hauteur du rostre :

0. approximativement égale à la hauteur de la boîte crânienne (sans la crête sagittale)

1. inférieure à la boîte crânienne mais supérieure à la moitié de la hauteur de la boîte crânienne

2. inférieure ou égale à la moitié de la hauteur de la boîte crânienne
68. Degré de la dilatation du rostre

0 . modéré

1. bas

2. élevé

69. Région centrale du nasal

0 . pas spécialement dilatée

1. nettement dilatée

70. Foramens palatins antérieurs

0 . fermés latéralement par les maxillaires

1. encerclés par les prémaxillaires

71. Prémaxillaires

0 . pas spécialement épais

1. très épais avec une crête distincte sur la surface dorsale de la ligne de contact

72. Forme du palais antéroventral

0 . V

1. V très ouvert

2. U

73. Extension postérieure de la ligne médiane du palais

0 . postérieure ou au même niveau que la M3

1. antérieure à la M3

74. Extension postérieure de la ligne médiane du palais

0 . postérieure ou égale au niveau de l'incisure postérolatérale du palais

1. antérieure au niveau de l'incisure postérolatérale du palais

75. Dépression du frontal

0 . profonde à nettement visible

1. absente ou peu profonde

76. Arêtes supra-orbitaires

0 . présentes

1. absentes

77. Développement des crêtes supra-orbitaires

0 . faible

1. fort

78. Position du foramen infra-orbitaire

0 . dorsale à M1-2

1. dorsale à $\mathrm{M} 2$

2. dorsale à M3

79. Forme du foramen infra-orbitaire

0 . ronde

1. quasi arrondie

2. allongée

80. Taille du foramen lacrimal

0 . petite

1. élargie

81. Bordure antéroventrale du plancher orbitaire

0 . arrondie

1. élevée et forte 
82. Largeur de l'os zygomatique comparée à la largeur maximum de la boîte crânienne

0 . inférieure ou égale
1. supérieure

83. Hauteur du processus zygomatique

0 . bas

1. de hauteur modérée

2. très haut

84. Crête sagittale

0 . basse

1. haute

85. Crête sagittale

0 . incomplète

1. complète

86. Pont sphénorbitaire

0 . comprimé (largeur postérieure au processus ptérygoïde équivalente aux trois quarts de la largeur antérieure)

1. large (les marges latérales postérieures au processus ptérygoïdes sont parallèles)

2. très comprimé (largeur postérieure au processus ptérygoïde équivalente à la moitié de la largeur antérieure)

3. considérablement comprimé (largeur postérieure au processus ptérygoïde nettement inférieure à la moitié de la largeur antérieure)

4. aucune compression

87. Fissure sphénorbitaire

0 . fissure et foramen optique petits, jumelés

1. fissure allongée

88. Extension postérieure de la fissure sphénorbitaire

0 . au niveau de la fosse glénoïde

1. antérieure à la fosse glénoïde

89. Foramen optique

0 . complètement séparé de la fissure sphénorbitaire par une barre osseuse

1. pas complètement séparé

2. confluent avec la fissure sphénorbitaire

90. Voûte mésoptérygoïde

0 . sans rainure

1. avec une rainure ouverte sur la dépression du basisphénoïde

2. avec une profonde et large rainure se terminant en une excavation antérieure au basisphénoïde (sans dépression)

91. Saillie du vomer postérieure au palais

0 . discrète

1. bien visible

92. Position du processus crochu du sphénoïde

0 . au tiers médian du pont sphénoïdal

1. postérieure au tiers

2. au milieu ou postérieure au tiers

3. antérieure au tiers
93. Fosse glénoïde

0 . arrondie

1. large (significativement plus large que long)

94. Dépression du basisphénoïde

0 . présente

1. absente

95. Forme du basisphénoïde

0 . longue et large

1. losangique

2. extrêmement courte

96. Expansion du pétrosal

0 . largeur de la cavité périotique égale à 6 fois la distance interpériotique (largeur du basioccipital)

1. 1 à 3 fois

2. 4 fois

3. 5 fois

4. 8 fois

97. Position du foramen oval au niveau

0 . antérieure à la zone médiane de la fosse glénoïde

1. postérieure à la zone médiane de la fosse glénoïde

98. Taille du foramen oval

0 . inférieure à la moitié de la surface du glénoïde

1. égale à la moitié ou au deux tiers de la surface du glénoïde

2. supérieure au deux tiers de la surface du glénoïde

99. Foramen accessoire au foramen oval (?suboval)

0 . absent

1. présent

100. Processus paroccipital

0 . mince

1. large, étendu latéralement

101. Longueur relative de l'inflation rostrale

0 . supérieure à la moitié de la longueur du rostre

1. inférieure ou égale à la moitié de la longueur du rostre (longueur du rostre $=$ de l'alvéole de la canine jusqu'à la largeur minimum interorbitaire)

102. Barre antéorbitaire

0 . peu ou pas réduite

1. nettement réduite

2. absente

103. Fosse latéroventrale

0 . présente

1. réduite ou absente

104. Longueur relative du rostre

0. égale ou supérieure à la moitié de la longueur de la boîte crânienne

1. inférieure à la moitié de la longueur de la boîte crânienne

105. Dilatation du rostre

0 . quadrant postérolatéral pas spécialement dilaté

1. quadrant postérolatéral très largement dilaté 
ANNEXE 2. - Matrice de caractères utilisée pour l'analyse phylogénétique incluant les Nycteridae et Emballonuridae (cf. Chapitre V). Les auteurs des taxons sont cités dans la dernière partie de l'Annexe 2 .

\begin{tabular}{|c|c|c|c|c|c|c|c|c|c|c|c|c|c|c|c|c|c|c|c|c|}
\hline Taxa & 1 & 2 & 3 & 4 & 5 & 6 & 7 & 8 & 9 & 10 & 11 & 12 & 13 & 14 & 15 & 16 & 17 & 18 & 19 & 20 \\
\hline Icaronycteris menui & ? & ? & 1 & ? & 0 & 0 & ? & ? & 0 & 0 & 0 & 0 & 1 & 0 & 0 & 0 & 0 & 0 & 0 & 1 \\
\hline Archaeonycteris brailloni & ? & ? & ? & ? & $?$ & 0 & ? & ? & 0 & 0 & 0 & 0 & 1 & 0 & 1 & 0 & 0 & 0 & 0 & 1 \\
\hline Palaeochiropteryx tupaiodon & 0 & 0 & 1 & 0 & 0 & 0 & 1 & 0 & 1 & 0 & 0 & 0 & 1 & 0 & 1 & 0 & 0 & 0 & 0 & 1 \\
\hline Nycteris grandis & 1 & 1 & 0 & 1 & 0 & 1 & 1 & 0 & 0 & 1 & 1 & 1 & - & - & 1 & 2 & 1 & 1 & 1 & 0 \\
\hline Nycteris gambiensis & 1 & 1 & 0 & 1 & 0 & 1 & 1 & 0 & 0 & 1 & 1 & 1 & - & - & 1 & 2 & 1 & 1 & 1 & 0 \\
\hline Chibanycteris herberti & ? & ? & ? & ? & ? & ? & ? & ? & ? & ? & ? & ? & ? & ? & ? & ? & ? & ? & 1 & ? \\
\hline Khoufechia gunnelli Ravel n. gen., n. sp. & ? & ? & ? & ? & ? & ? & ? & ? & ? & 0 & 1 & ? & ? & ? & 1 & 1 & 1 & 1 & 1 & 0 \\
\hline Dhofarella thaleri & ? & ? & ? & ? & ? & ? & ? & ? & ? & ? & ? & ? & ? & ? & 0 & 0 & 1 & 1 & 0 & 0 \\
\hline Vespertiliavus wingei & 0 & 0 & 1 & 1 & 0 & 0 & ? & ? & 1 & 0 & 0 & 1 & 1 & 1 & 0 & 0 & 1 & 1 & 0 & 0 \\
\hline ?Vespertiliavus aenigma Ravel, n. sp. & ? & ? & ? & ? & ? & ? & ? & ? & 1 & 0 & 0 & ? & ? & ? & 0 & 0 & 1 & 1 & 0 & 1 \\
\hline Vespertiliavus kasserinensis Ravel, n. sp. & ? & ? & ? & ? & $?$ & ? & ? & ? & ? & ? & ? & ? & 1 & 1 & ? & ? & ? & ? & 0 & 1 \\
\hline Tachypteron franzeni & 0 & 0 & 0 & 0 & 0 & 0 & 1 & 1 & 1 & 0 & 1 & 0 & 1 & 1 & 0 & 0 & 0 & 1 & 0 & ? \\
\hline Emballonura atrata & 0 & 0 & 0 & 0 & 0 & 1 & 1 & 2 & 0 & 0 & 0 & 0 & - & - & 0 & 0 & 1 & 1 & 0 & 1 \\
\hline Taphozous melanopogon & 0 & 0 & 1 & 0 & 1 & 1 & 1 & 0 & 0 & 0 & 0 & 1 & - & - & 0 & 0 & 1 & 1 & 0 & 0 \\
\hline $\begin{array}{l}\text { Pseudovespertiliavus parva Ravel n. gen., } \\
\text { n. sp. }\end{array}$ & ? & ? & ? & ? & ? & ? & ? & ? & ? & ? & ? & ? & ? & ? & ? & ? & ? & ? & 0 & 1 \\
\hline $\begin{array}{l}\text { Chambinycteris pusilli Ravel n. gen., } \\
\text { n. sp. }\end{array}$ & 0 & 1 & 1 & 0 & 1 & 0 & $?$ & ? & 1 & 0 & 1 & ? & 0 & ? & ? & ? & ? & ? & 0 & ? \\
\hline Vespertiliavus schlosseri & ? & ? & ? & ? & $?$ & ? & ? & ? & 1 & 0 & 0 & 1 & ? & ? & $0+1$ & 0 & 0 & 1 & 0 & 1 \\
\hline Vespertiliavus gracilis & ? & ? & ? & ? & 0 & 0 & ? & ? & 1 & 0 & 0 & 1 & 1 & 1 & 0 & 0 & 1 & 1 & 0 & 1 \\
\hline
\end{tabular}

Taxa

$\begin{array}{llllllllllllllllllll}21 & 22 & 23 & 24 & 25 & 26 & 27 & 28 & 29 & 30 & 31 & 32 & 33 & 34 & 35 & 36 & 37 & 38 & 39 & 40\end{array}$

Icaronycteris menui

$\begin{array}{llllllllllllllllllll}1 & 0 & 0 & 0 & 2 & 2 & 0 & 0 & 0+1 & 0 & 0 & 0 & 0 & 1 & 1 & 0 & 1 & 2 & 0 & 2\end{array}$

Archaeonycteris brailloni

Palaeochiropteryx tupaiodon

Nycteris grandis

Nycteris gambiensis

Chibanycteris herberti

Khoufechia gunnelli

Ravel n. gen., n. sp.

Dhofarella thaleri

Vespertiliavus wingei

?Vespertiliavus aenigma Ravel, n. sp.

Vespertiliavus kasserinensis Ravel, n. sp.

Tachypteron franzeni

Emballonura atrata

Taphozous melanopogon

Pseudovespertiliavus parva

Ravel n. gen., n. sp.

Chambinycteris pusilli

Ravel n. gen., n. sp.

Vespertiliavus schlosseri

Vespertiliavus gracilis

$\begin{array}{cccccccccccccccccccc}1 & 0 & 0 & 0 & 2 & 2 & 0 & 0 & 0+1 & 0 & ? & ? & 0 & 1 & 0+1 & ? & 1 & 2 & 0 & 2 \\ 1 & 0 & 0 & 0 & 0 & 2 & 0 & 0 & 1 & 1 & 0 & 0 & 0 & 1 & 0 & 0 & 1 & 2 & 1 & 0 \\ 1 & 1 & 1 & 1 & 1 & 1 & 0 & 1 & 1 & 1 & 1 & 0 & 2 & 0 & 0 & 2 & 0 & 1 & 1 & 0 \\ 0 & 1 & 1 & 1 & 1 & 1 & 0 & 1 & 1 & 1 & 1 & 0 & 1 & 0 & 1 & 2 & 0 & 2 & 1 & 0 \\ ? & ? & ? & 1 & ? & ? & ? & ? & ? & ? & 1 & 0 & ? & ? & ? & ? & ? & ? & ? & 0 \\ 0 & 1 & 1 & 0 & 2 & 1 & 0 & 1 & 1 & 1 & 1 & 1 & 0 & 1 & 1 & ? & 0 & 2 & 1 & 0\end{array}$

Taxa

$\begin{array}{lllllllllllllllllllllll} & 0 & 0 & ? & ? & 0 & 0 & 1 & 0 & 2 & 0 & ? & ? & ? & ? & ? & ? & ? & ? & ? & 0\end{array}$

$\begin{array}{lllllllllllllllllllll}1 & 0 & 0 & 0 & 0 & 0 & 0 & 0 & 2 & 0 & 1 & 1 & ? & ? & ? & ? & 1 & 1 & 1 & 1\end{array}$

$\begin{array}{cccccccccccccccccccc}1 & 0 & 0 & ? & 0 & 0 & 0 & 0 & 2 & 0 & ? & ? & 1 & 1 & 0 & ? & 1 & 1 & 1 & 0+1 \\ 1 & 0 & 0 & 0 & 0 & 0 & 1 & 0 & 2 & 0 & 1 & 1 & 0 & 1 & 0 & ? & 1 & 0+1 & 1 & 1\end{array}$

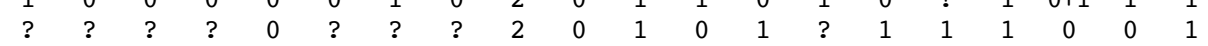

$\begin{array}{llllllllllllllllllll}1 & 0 & 0 & 0 & 0 & 0 & 1 & 0 & 2 & 0 & 1 & 0 & 0 & 0 & 0 & 1 & 1 & 0 & 0 & 0 \\ 1 & 1 & 0 & 0 & 0 & 0 & 1 & 1 & 2 & 0 & 1 & 1 & 0 & 1 & 0 & 1 & 1 & 0 & 1 & 0\end{array}$

$\begin{array}{llllllllllllllllllll}1 & 1 & 0 & 0 & 0 & 2 & 0 & 1 & 2 & 0+1 & 1 & 0 & 0 & 1 & 0 & ? & ? & ? & ? & 0+1\end{array}$

Icaronycteris menui

Archaeonycteris brailloni

Palaeochiropteryx tupaiodon

Nycteris grandis

Nycteris gambiensis

Chibanycteris herberti

Khoufechia gunnelli

Ravel n. gen., n. sp.

Dhofarella thaleri

Vespertiliavus wingei

?Vespertiliavus aenigma Ravel, n. sp.

Vespertiliavus kasserinensis Ravel, n. sp.

Tachypteron franzeni

Emballonura atrata

Taphozous melanopogon

Pseudovespertiliavus parva

Ravel n. gen., n. sp.

Chambinycteris pusilli

Ravel n. gen., n. sp.

Vespertiliavus schlosseri

$\begin{array}{llllllllllllllllllllll}1 & ? & 0 & ? & 0 & 2 & 0 & 0 & 2 & 0 & ? & ? & 0 & 1 & 1 & 0 & 1 & ? & ? & 0\end{array}$

Vespertiliavus gracilis

$\begin{array}{llllllllllllllllllll}1 & 0 & 0 & 0 & 0 & 0 & 0 & 0 & 2 & 0 & 1 & 1 & 1 & 1 & 0 & ? & 1 & 1 & 1 & 1 \\ 1 & 0 & 0 & 0 & 0 & 0 & 1 & 0 & 2 & 0 & 1 & 1 & 1 & 1 & 0 & ? & 1 & 1 & 1 & 1\end{array}$

$\begin{array}{cccccccccccccccccccc}\mathbf{4 1} & \mathbf{4 2} & \mathbf{4 3} & \mathbf{4 4} & \mathbf{4 5} & \mathbf{4 6} & \mathbf{4 7} & \mathbf{4 8} & \mathbf{4 9} & \mathbf{5 0} & \mathbf{5 1} & \mathbf{5 2} & \mathbf{5 3} & \mathbf{5 4} & \mathbf{5 5} & \mathbf{5 6} & \mathbf{5 7} & \mathbf{5 8} & \mathbf{5 9} & \mathbf{6 0} \\ 0 & 1 & 0 & 1 & 0 & 0 & 0 & 0 & 0 & 1 & 0 & 0 & 0 & 1 & 0 & 1 & 2 & 2 & 0 & 1 \\ 0 & 1 & 0 & 1 & 0 & 0 & 0 & 0 & 0 & 0 & 0 & 0 & 0 & 1 & 0 & 1 & 2 & 2 & 0 & 1 \\ 0 & 1 & 0 & 1 & 0 & 0 & 0 & 0 & 0 & 1 & 0 & 0 & 0 & 1 & 0 & 1 & 2 & 2 & 0 & 1 \\ 0 & 1 & 1 & 0 & 1 & 0 & 1 & 1 & 1 & 1 & 1 & 2 & 1 & 1 & 0 & 1 & 2 & 0 & 0 & 0 \\ 0 & 1 & 1 & 0 & 1 & 0 & 1 & 1 & 1 & 1 & 1 & 2 & 1 & 1 & 0 & 1 & 2 & 0 & 0 & 0 \\ 0 & ? & 0 & 0 & 1 & ? & 1 & 1 & 1 & 1 & 1 & 2 & 1 & 1 & 0 & 1 & 2 & 0 & 0 & 0 \\ 0 & 1 & 1 & 0 & 1 & 0 & 0+1 & 1 & 1 & 1 & 0 & 2 & 1 & 1 & 0 & 1 & 2 & 0 & 0 & 1\end{array}$

$\begin{array}{llllllllllllllllllll}1 & 0 & 1 & 0 & 0 & 1 & 1 & 1 & 0 & 0 & 0 & 1 & 0 & 0 & 1 & 0 & 2 & 1 & 0 & 0\end{array}$ $\begin{array}{llllllllllllllllllll}1 & 0 & 1 & 0 & 0 & 0 & 1 & 1 & 0 & 1 & 0 & 1 & 0 & 0 & 1 & 1 & 2 & 1 & 0 & 0 \\ 1 & 0 & 1 & 0 & 0 & 0 & 1 & 1 & 0 & 1 & 0 & 0+1 & 0 & 0 & 1 & 0 & 2 & 1 & 0 & 0\end{array}$ $\begin{array}{cccccccccccccccccccc}1 & 0 & 1 & 0 & ? & 0+1 & 0+1 & 0+1 & 0 & 1 & 0 & 1 & 0 & 0 & 1 & 0 & 1+2 & 1 & 1 & 0\end{array}$ $\begin{array}{llllllllllllllllllll}1 & 0 & 1 & ? & 0 & 0 & 1 & 1 & 0 & 1 & 0 & 2 & 0 & 0 & 0 & 1 & 2 & 1 & 0 & 1\end{array}$ $\begin{array}{llllllllllllllllllll}1 & 0 & 1 & 0 & 1 & 1 & 0 & 0 & 0 & 0 & 0 & 1 & 0 & 0 & 1 & 0 & 0 & 1 & 1 & 1 \\ 1 & 0 & 1 & 0 & 1 & 1 & 1 & 1 & 1 & 1 & 0 & 1 & 1 & 0 & 1 & 0 & 2 & 1 & 0 & 0\end{array}$ $\begin{array}{cccccccccccccccccccc}1 & 0 & 1 & 0 & 1 & 1 & 1 & 1 & 1 & 1 & 0 & 1 & 1 & 0 & 1 & 0 & 2 & 1 & 0 & 0 \\ 1 & 0 & 1 & 0 & 1 & 0+1 & 0+1 & 1 & 0 & 1 & 0 & 2 & 0 & 0 & 1 & 1 & 1+2 & 0+1 & 0 & 1\end{array}$ $\begin{array}{lllllllllllllllllllll}1 & 0 & 1 & 0 & 1 & 0 & 0 & 1 & 1 & 1 & 0 & 2 & 0 & 1 & 0 & 1 & 1 & 2 & 0 & 1\end{array}$ $\begin{array}{cccccccccccccccccccc}1 & 0 & 1 & 0 & 1 & 0 & 1 & 1 & 0+1 & 1 & 0 & 1+2 & 0 & 0 & 1 & 1 & 2 & 1 & 0 & 0 \\ 1 & 0 & 1 & 0 & 1 & 0 & 1 & 1 & 1 & 1 & 0 & 1 & 0 & 0 & 1 & 0 & 2 & 1 & 1 & 0\end{array}$ 
ANNEXE 2. - Suite. Les auteurs des taxons de l'Annexe 2 sont cités ici.

\begin{tabular}{|c|c|c|c|c|c|}
\hline Taxa & 61 & 62 & 63 & 64 & 65 \\
\hline Icaronycteris menui Russell, Louis \& Savage, 1973 & 0 & 0 & 1 & 0 & 0 \\
\hline Archaeonycteris brailloni Russell, Louis \& Savage, 1973 & 0 & ? & ? & ? & ? \\
\hline Palaeochiropteryx tupaiodon Revilliod, 1917 & 0 & 0 & 0 & 0 & 0 \\
\hline Nycteris grandis Peters, 1865 & 1 & 1 & 2 & 1 & 0 \\
\hline Nycteris gambiensis Andersen, 1912 & 1 & 1 & 2 & 1 & 0 \\
\hline Chibanycteris herberti Sigé, Thomas, Sen, Gheerbrant, Roger \& Al-Sulaimani, 1994 & 0 & ? & ? & ? & ? \\
\hline Khoufechia gunnelli Ravel n. gen., n. sp. & 1 & 1 & 0 & 1 & 0 \\
\hline Dhofarella thaleri Sigé, Thomas, Sen, Gheerbrant, Roger \& Al- Sulaimani, 1994 & 0 & ? & ? & ? & ? \\
\hline Vespertiliavus wingei Revilliod, 1920 & 0 & 0 & 0 & 1 & 0 \\
\hline ?Vespertiliavus aenigma Ravel, n. sp. & 0 & 0 & 1 & 1 & 0 \\
\hline Vespertiliavus kasserinensis Ravel, $\mathrm{n}$. sp. & 0 & 0 & 1 & 1 & 0 \\
\hline Tachypteron franzeni Storch, 2002 & 0 & 0 & 0 & 0 & 0 \\
\hline Emballonura atrata Peters, 1874 & 0 & 0 & 1 & 1 & 0 \\
\hline Taphozous melanopogon Temminck, 1841 & 0 & 0 & 2 & 1 & 1 \\
\hline Pseudovespertiliavus parva Ravel n. gen., n. sp. & $0+1$ & ? & ? & ? & ? \\
\hline Chambinycteris pusilli Ravel n. gen., n. sp. & 0 & $?$ & $?$ & ? & $?$ \\
\hline Vespertiliavus schlosseri Revilliod, 1920 & 0 & 0 & 0 & 1 & 0 \\
\hline Vespertiliavus gracilis Revilliod, 1920 & 0 & 0 & 1 & 1 & 0 \\
\hline
\end{tabular}

Description des caractères de la matrice de l'Annexe 2.

OS DENTAIRE

\section{Processus coronoïde}

0 . haut (environ deux fois la hauteur de la rangée dentaire)

1. bas

2. Apex du processus coronoïde

0 . pointu

1. arrondi

3. Ramus horizontal

0 . gracile (moins élevé que la hauteur des molaires [couronne])

1. robuste (hauteur supérieure à celle des molaire [couronne])

4. Branche montante du processus coronoïde

0 . quasi perpendiculaire au ramus horizontal

1. incliné distalement

5. Nombre d'incisives inférieures

0 . trois incisives sur chaque mandibule

1. deux incisives sur chaque mandibule

6. Nombre de prémolaires inférieures sur chaque mandibule

0 . trois prémolaires sur chaque mandibule

1. deux prémolaires sur chaque mandibule

DENTURE INFÉRIEURE

7. Incisives inférieures
0 . bilobées
1. trilobées

8. Taille relative des incisives inférieures

0 . incisives de taille équivalente

1. i3 est la plus large des incisives inférieures

2. i1 est la plus large des incisives inférieures
9. Cingulum buccal de $\mathrm{c} 1$

0 . bien individualisé et continu

1. très fin voir absent

10. Tubercule de c1

0 . droit

1. recourbé distalement

11. Bassin distal de $\mathrm{cl}$

0 . étendu distalement

1. réduit

12. $\mathrm{p} 2$

0 . réduite

1. non réduite (taille équivalente à $\mathrm{p} 4$ )

13. $\mathrm{p} 3$

0 . uniradiculée

1. biradiculée

14. Couronne de $\mathrm{p} 3$

0 . bien développée

1. très réduite

15. Développement de la $\mathrm{p} 4$

0 . bien développé (taille supérieure à la prémolaire antérieure, munie d'un protoconide et d'un talonide)

1. réduite (taille inférieure à la prémolaire antérieure)

16. Talonide sur $\mathrm{p} 4$

0 . étendu distalement

1. réduit

2. absent

17. Paraconide sur $\mathrm{p} 4$

0 . présent

1. absent 
18. Métaconide sur $\mathrm{p} 4$

0 . présent

1. absent

19. Cuspides du trigonide

0 . hautes et bien individualisées

1. peu élevées

20. Triangle du trigonide de $\mathrm{m} 1$

0 . ouvert lingualement

1. comprimé mésiodistalement

21. Triangle du trigonide de $\mathrm{m} 2$

0 . ouvert lingualement

1. comprimé mésiodistalement

22. Distance entre le paraconide et le métaconide sur $\mathrm{m} 1$

0 . inférieure à la distance entre le métaconide et l'entoconide

1. supérieure ou égale à la distance entre le métaconide et l'entoconide

23. Distance entre le paraconide et le métaconide sur $\mathrm{m} 2$

0 . inférieure à la distance entre le métaconide et l'entoconide

1. supérieure ou égale à la distance entre le métaconide et l'entoconide

24. Distance entre le paraconide et le métaconide sur $\mathrm{m} 3$

0 . inférieure à la distance entre le métaconide et l'entoconide

1. supérieure ou égale à la distance entre le métaconide et l'entoconide

25. Largeur du trigonide

0 . inférieure à celle du talonide

1. supérieure à celle du talonide

2. équivalente à la largeur du talonide

26. Entoconide

0 . haut et incliné distalement

1. absent à très réduit

2. modéré et droit

27. Entocristide de m1-2

0 . rectiligne en vue occlusale

1. sinueuse en vue occlusale

28. Orientation de la cristide oblique

0 . rejoignant la paroi distale du trigonide au niveau de la jonction entre la métacristide et la postprotocristide

1. rejoignant la paroi distale du trigonide à un niveau plus lingual que la jonction entre la métacristide et la postprotocristide

29. Position de l'hypoconulide

0 . hypoconulide en position médiane et distale entre l'entoconide et l'hypoconide

1. éloignée et légèrement plus buccale que l'entoconide

2. très proche et distale à l'entoconide
30. Postcristide

0 . rectiligne

1. incurvée distalement

31. Largeur du talonide de $\mathrm{m} 3$

0 . équivalente à la largeur du trigonide

1. inférieure à celle du trigonide

32. Hypoconulide sur $\mathrm{m} 3$

0 . présent

1. absent

DENTURE SUPÉRIEURE

33. Cingulum buccal sur $\mathrm{C} 1$
0 . présent
1. absent
2. très fin et discontinu

34. Cingulum lingual de $\mathrm{C} 1$

0 . fortement incurvé

1. quasi rectiligne

35. Ressaut postérieur du cingulum sur $\mathrm{C} 1$

0 . présent

1. absent

36. Nombre de prémolaires supérieures sur chaque mandibule

0 . trois prémolaires supérieures

1. deux prémolaires supérieures

2. une seule prémolaire supérieure

37. Bassin du talon de P4

0 . étiré lingualement

1. comprimé buccolingualement

38. Couronne de P4

0 . munie d'un lobe buccomésial prononcé

1. sans lobe buccomésial

2. munid'un faible lobe buccomésial

39. Tubercule mésiolinguale sur P4

0 . présent

1. absent

40. Ectoflexus

0 . double

1. simple et antérieure au mésostyle

2. simple et central

41. Dissymétrie des « $\mathrm{V} »$ de l'ectolophe

0 . faible

1. prononcée

42. Inclinaison du bord buccal de M1

0 . forte

1. faible

43. Position du mésostyle

0 . en retrait par rapport au bord buccal

1. projeté sur le bord buccal 
44. Cingulum buccal de M1-2

0 . discontinu le long de la bordure buccale

1. continu le long de la bordure buccale

45. Cingulum buccal au niveau du mésostyle

0 . présent

1. absent

46. Parastyle de M1

0 . connecté à la préparacrête

1. isolé

47. Paralophe

0 . présent

1. absent

48. Métalophe

0 . présent

1. absent

49. Précingulum

$0 . \quad$ large

1. très fin à absent

50. Postcingulum

$0 . \quad$ large

1. fin

51. Protocône

0 . bien développé (volume supérieur au métacône et au paracône)

1. réduit (volume égal ou inférieur au métacône et au paracône)

52. Extension de la postprotocrête

0 . rejoint le postcingulum

1. rejoint le cingulum lingual ou l'hypocône

2. ne rejoint aucune autre structure

53. Orientation de la préprotocrête

0 . se connecte au précingulum

1. rejoint la base du paracône

54. Protofosse

0 . étendue selon l'axe mésiodistal

1. courte
55. Ouverture distale de la protofosse

0 . protofosse clôturée distalement par la postprotocrête

1. protofosse ouverte

56. Niveau de la protofosse

0 . basse et s'ouvrant sur le bassin du talon

1. haute et sans connexion franche avec le bassin du talon

57. Hypocône

0 . présent et bien développé

1. présent mais succinct

2. absent

58. Aplanissement distal du talon

0 . fort

1. faible

2. absent

59. Bordure distale du talon

0 . arrondie

1. munie d'un lobe distolingual

60. Orientation du talon

0 . distale

1. linguodistale

61. Cingulum lingual

$0 . \quad$ épais

1. fin

62. Développement de M3

0 . fort (plus de la moitié de la largeur de la M2)

1. faible (moins de la moitié de la largeur de M2)

63. Métacône de M3

0 . petit

1. bien développé (de volume équivalent au paracône)

2. absent

64. Cingulum lingual de M3

0 . présent

1. absent

65. Prémétacrête sur M3

0 . présente

1. absente 